Claremont Colleges

Scholarship @ Claremont

Spring 2021

\title{
Build it so they will come? Feasibility and efficacy of a gamified personalized normative feedback alcohol intervention for sexual minority women.
}

Sarah C. Boyle

Claremont Graduate University

Follow this and additional works at: https://scholarship.claremont.edu/cgu_etd

\section{Recommended Citation}

Boyle, Sarah C.. (2021). Build it so they will come? Feasibility and efficacy of a gamified personalized normative feedback alcohol intervention for sexual minority women.. CGU Theses \& Dissertations, 202. https://scholarship.claremont.edu/cgu_etd/202. doi: 10.5642/cguetd/202

This Open Access Dissertation is brought to you for free and open access by the CGU Student Scholarship at Scholarship @ Claremont. It has been accepted for inclusion in CGU Theses \& Dissertations by an authorized administrator of Scholarship @ Claremont. For more information, please contact scholarship@cuc.claremont.edu. 
Build it so they will come? Feasibility and efficacy of a gamified personalized normative feedback alcohol intervention for sexual minority women.

\author{
by \\ Sarah C. Boyle
}

Claremont Graduate University

2021 
(C) Copyright Sarah C. Boyle, 2021. All rights reserved. 


\section{APPROVAL OF THE DISSERTATION COMMITTEE}

This dissertation has been duly read, reviewed, and critiqued by the Committee listed below, which hereby approves the manuscript of Sarah C. Boyle as fulfilling the scope and quality requirements for meriting the degree of Doctor of Philosophy in Psychology with a concentration in Social Psychology.

Allen M. Omoto, Chair

Pitzer College

Vice President for Academic Affairs and Dean of Faculty

William D. Crano

Claremont Graduate University

Oskamp Distinguished Professor of Psychology

Jason T. Siegel

Claremont Graduate University

Professor of Psychology

Robin J. Lewis

Old Dominion University

Professor of Psychology 


\begin{abstract}
Build it so they will come? Feasibility and efficacy of a gamified personalized normative feedback alcohol intervention for sexual minority women

Sarah C. Boyle
\end{abstract}

Claremont Graduate University: 2021

Sexual minority women (SMW) disproportionately engage in heavy drinking and shoulder the burden of alcohol dependence. Much research has emphasized the need for culturally tailored alcohol interventions for this population, highlighting sexual minority stigmarelated experiences, maladaptive coping, and misperceived peer drinking and coping norms as potential intervention targets. Focusing on the latter, this research examines the potential utility of personalized normative feedback (PNF) in reducing consumption among moderate and heavy drinking SMW. PNF is a popular, evidence-based intervention strategy associated with reliable (albeit modest) reductions in alcohol use in other heavy drinking populations. To remedy limitations associated with traditional PNF intervention formats and tailor this strategy to reflect the interests, social identities, and stigma experiences of SMW, a novel gamified intervention format was developed wherein PNF on drinking and coping behaviors was organically delivered to SMW within LezParlay, a larger competition designed to challenge negative stereotypes about lesbian, bisexual, queer (LBQ) women and increase the visibility of community members. The current study evaluates the efficacy and feasibility of this approach.

In total, 2,677 LBQ women between the ages of 21 and 65 years signed up to take part in the LezParlay competition, with players logging over 44,0000 web app page views. Following several rounds of play, a sub-sample of 499 moderate-to-heavy drinking SMW were randomized to receive 1 of 3 sequences of PNF (i.e., alcohol+coping, alcohol+control, or control topics only) 
over two subsequent rounds. Alcohol use was assessed prior to randomization and two months post-intervention, along with potential demographic and sexual minority stigma-related moderators. Following the competition, these participants completed feedback surveys assessing acceptability, perceived benefits, and ideas for future versions of LezParlay.

At follow-up, participants who received alcohol+coping and alcohol+control PNF significantly reduced their alcohol consumption relative to those who received control PNF only $(d=.49-.50)$. No differences were observed between treatment conditions overall; however, moderator analyses revealed alcohol+coping PNF to be more effective than alcohol only PNF among SMW who entered the study as heavier drinkers. Interpersonal stigma exposure also moderated intervention efficacy with enhanced effects observed among SMW in both treatment conditions reporting greater (relative to lesser) violence and harassment due to sexual minority status. Study participants found the competition to be highly acceptable and $93 \%$ reported psychological benefits, which most frequently included stigma reduction, social comparison, community connection, entertainment, self-confrontation, and mood enhancement.

Findings support the feasibility and efficacy of this palatable approach to alcohol intervention for SMW, suggest that culturally tailored game mechanics may bolster PNF intervention engagement and potentially carry psychological benefits beyond core intervention content, and illuminate several priority directions for future research. Foremost, as findings suggest that PNF may particularly risk-reducing in the context of severe interpersonal stigma experiences like violence and harassment due to sexual minority status, additional research with SMW and members of other stigmatized groups is needed to more extensively examine potential interactions between norm correcting PNF and sexual minority stigma experiences in the context of heavy drinking and other health-risk behaviors. More broadly, this research advances several 
new directions for PNF intervention research, demonstrates the efficiency of hybrid feasibility/efficacy trial designs for evaluating digital health interventions, and illuminates the potential utility of incognito digital health intervention formats for nonclinical populations. 


\section{Acknowledgments}

I would like to express my sincere gratitude to the members of my dissertation committee, foremost my formal Research Advisor and Committee Chair, Dr. Allen Omoto. My writing skills and critical thinking in the LGBTQ health domain would certainly not be as advanced as they are without your encouraging of my potential while simultaneously challenging me to push the bar higher. I also sincerely appreciate you not dissuading me from seeking grant funding or embarking on a project of this size and scope for my dissertation. Also deserving of special thanks is my informal research mentor and colleague at Loyola Marymount University, Dr. Joseph LaBrie, who has encouraged my often crazy and oversized research ideas and ensured it was financially possible for me to seek grant-funding for them as a doctoral student. Joe, your mentorship and encouragement have been truly invaluable to me over the past six years, and I am extremely proud of our ongoing, highly innovative program of research. Committee members Dr. William Crano and Dr. Jason Siegel also deserve thanks. Although my work with Allen and Joe did not leave me time to be involved in your labs, the research, theory, and practical insights I was exposed to in your respective courses undoubtably shaped the present intervention's packaging as well as the trial's design and assessments. I am also grateful for the involvement of Robin Lewis as the external member of my committee. Robin, as I have long admired your research with sexual minority women from afar, it was truly special to have you be a part of this committee. I sincerely appreciate your feedback and time.

I also want to thank my partner and family for their encouragement and emotional support along the way. Sophia, I am grateful to have you in my life and am truly indebted to you for the love, strength, and understanding you continue to provide me. Not only my emotional rock, you also helped me with recruitment and enthusiastically acted as the project's guinea pig, 
serving as the first individual to test each round of LezParlay and providing feedback on question wording and the design and functionality of app screens. Your feedback and support played instrumental roles in the success of this project and I could not have done it without you. Finally, I would like to acknowledge that this research was supported by Developmental/ Exploratory Grant R21AA025767-01A from the National Institute on Alcohol Abuse and Alcoholism. 


\section{Table of Contents}

Introduction 1

Chapter 1 Literature Review.................................................. 5

1.1 Sexual orientation-based alcohol use disparities................................ 5

1.1 Explanations and implications for intervention............................. 6

1.2 Examining the utility of PNF for SMW: Unanswered questions.................. 16

Chapter 2 Intervention Development.............................................. 27

2.1 PNF intervention modalities, limitations, and challenges....................... 27

2.2 PNF intervention development considerations for SMW ........................ 28

2.3 A gamified, culturally tailored, incognito alcohol intervention for SMW .............. 36

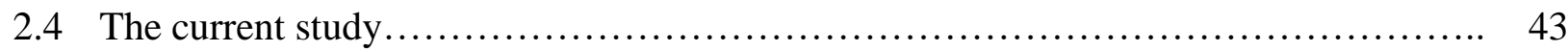

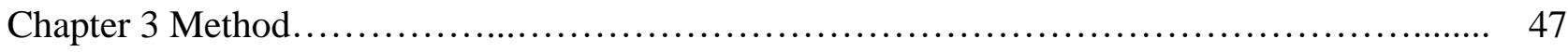

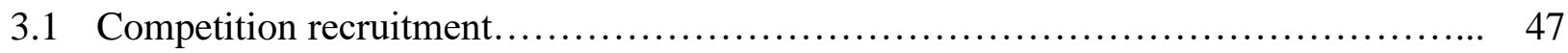

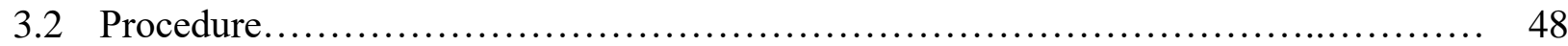

3.3 Measures............................................................. 56

Chapter 4 Efficacy Results................................................. 62

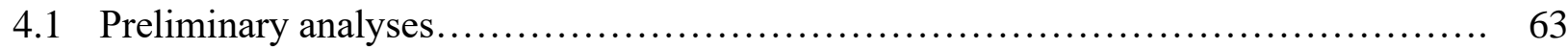

4.2 Main effects........................................................ 73

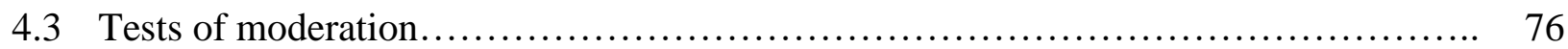

4.4 Summary of efficacy findings.......................................... 90

Chapter 5 Feasibility Results................................................ 87

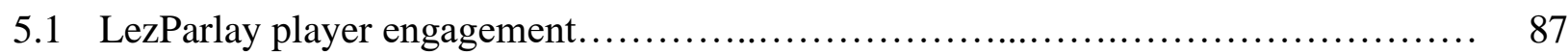

5.2 Alcohol consumption among Round 3 players.............................. 95

5.3 Acceptability among study participants................................. 96

5.4 Perceived benefits among study participants............................... 97

5.5 Participants' ideas \& requests for future versions.............................. 103

5.6 Summary of feasibility findings...................................... 104

Chapter 6 Discussion..................................................... 106

6.1 Overview of findings............................................... 106

6.2 Limitations and future directions......................................... 123

6.3 Broader implications for research and practice............................. 133 


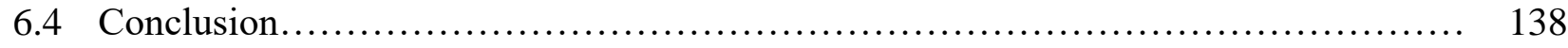

Appendix A: Recruitment \& promotional materials.................................. 139

Appendix B: Consent forms \& evaluation study invite............................... 145

Appendix C: Email from minor dropped from evaluation study........................ 153

Appendix D: Preliminary analysis of structural stigma indices.......................... 155

Appendix E: Supplemental results and model information............................. 159

Appendix F: Feedback survey \& debriefing statement................................ 169

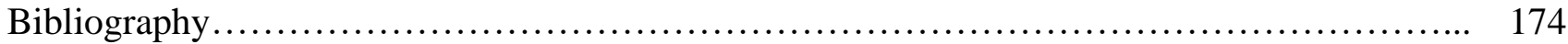




\section{INTRODUCTION}

Of all deaths worldwide, $5.9 \%$ are attributable to alcohol consumption, exceeding the proportion of global deaths attributable to HIV/AIDS, violence, and tuberculosis combined (World Health Organization, 2014). In the United States, an estimated 95,0000 people die annually from alcohol-related causes, positioning excessive alcohol consumption as the third leading preventable cause of death (National Institute on Alcohol Abuse \& Alcoholism, 2020). Further, excessive drinking represents an expensive public health issue, costing the U.S. economy 249 billion dollars each year in healthcare, disability, crime, diminished workplace productivity, and unemployment costs (Centers for Disease Control, 2015). The majority of these costs are attributable to the societal prevalence of alcohol use disorder (AUD), which is defined as a maladaptive pattern of excessive alcohol consumption leading to clinically significant impairment or distress (National Institute on Alcohol Abuse \& Alcoholism, 2020).

Public health tracking of AUD in the U.S. population is made possible by data from large-scale, representative, population-based surveys such as the National Epidemiologic Survey on Alcohol and Related Conditions (NESARC), which assesses alcohol-related health problems, treatment-seeking, and AUD symptoms that define non, mild, moderate and severe cases (National Institute of Alcoholism and Alcohol Abuse, 2020). NESARC data reveal that roughly 15 million U.S. adults meet the minimum diagnostic criteria for AUD per year and roughly onethird of all U.S. adults will meet these criteria at some point in their lifetimes (Grant et al., 2015). Studies have also highlighted increases in the prevalence of AUD in recent years (e.g., Mokdad et al., 2018), underscoring urgent needs for novel treatment approaches as well as communitybased intervention and prevention programs designed to increase awareness around problematic levels of consumption, reduce the escalations in drinking that lead to AUD, and motivate individuals engaging in excessive consumption to seek treatment. 
Analysis of NESARC data also reveal that neither AUD nor treatment/intervention availability are evenly distributed across the US population. Instead, the health of some subgroups of Americans are disproportionately impacted by alcohol, including individuals lower in socio-economic status (e.g., Collins et al., 2016) and minority groups marginalized in society (e.g., Hughes, 2011; Keyes, Liu, \& Cerda, 2012). Despite increased alcohol-related risks among these individuals, culturally tailored alcohol interventions and treatment options for these groups are often not available (Mulia, Tam, \& Schmidt, 2014; Vaeth, Wang-Schweig, \& Caetano, 2017). Such is the case among sexual minority women (SMW), a marginalized female subpopulation including those who psychologically identify as lesbian, bisexual, or queer in addition to those who report having sex with women and/or experiencing same-sex attraction (Sell, 1997; Talley, Sher, Steinley, Wood, \& Littlefield, 2012). Relative to heterosexual women, SMW disproportionately engage in heavy drinking, meet criteria for AUD, and suffer from alcoholrelated health problems (for reviews, see Hughes, 2011; Hughes, Veldhuis, Drabble, \& Wilsnack, 2020). Further, although heavy drinking is among the most prominent and welldocumented sexual-orientation-based health disparities among women, culturally tailored alcohol treatment, intervention, and prevention programs for SMW have been slow to emerge (Blume, 2016).

Fortunately, several programs are currently being developed to address this disparity in alcohol intervention research and practice. In the U.S., Pachankis and colleagues (2020) are developing and pilot testing an intensive, cognitive-behavioral program that addresses sexual minority stigma-related processes and jointly targets depression, anxiety, and alcohol consumption among heavy drinking SMW presenting with comorbid mental health issues. Meanwhile, in Australia, Bush and colleagues (2019) are developing a text-message based 
intervention for SMW with AUD which delivers supportive messages focused on drinking reduction goal setting, healthy stigma-coping, and connection to local treatment services. Commonalities between these programs include their focus on alcohol use and poor mental health, attention to stigma-coping responses, and their orientation toward SMW seeking culturally tailored AUD treatment and those motivated to reduce their alcohol consumption.

However, NESARC data reveal that only roughly $10 \%$ of heavy drinkers in the U.S. that meet diagnostic criteria for AUD ever seek treatment, illuminating what is known in this literature as the "treatment gap" (Cohen, Feinn, Arias, \& Kranzler, 2007; National Institute of Alcohol Abuse \& Alcoholism, 2020;). Research investigating reasons for not seeking treatment among individuals meeting criteria for AUD reveal that treatment barriers are more frequently attitudinal (e.g., belief that consumption levels are not problematic) or motivational (e.g., not wanting to reduce one's drinking) than structural (e.g., lack of access, inability to afford costs; Grant, 1997; May \& Nielsen, 2019; Saunders, Zygowicz, \& D’Angelo, 2006). As bars and nightclubs occupy central physical spaces in LGB communities and are characterized by permissive substance use norms (e.g., Cochran, Grella, \& Mays, 2012; Green \& Feinstein, 2012), several studies have suggested that failing to recognize one's drinking as problematic and lacking the motivation to change may similarly act as major barriers to intensive alcohol interventions and treatment programs among sexual minorities (e.g., Allen \& Mowbray, 2016; Green, 2011; Levak, Kuerbis, \& Morganstern, 2020).

In light of the treatment gap, the present research advances and evaluates a novel, evidence-based digital alcohol intervention designed to reduce alcohol use and related problems among alcohol consuming lesbian, bisexual, and queer-identified SMW not seeking treatment for alcohol use disorder. The "incognito" intervention approach introduced herein is uniquely 
oriented toward engaging and preventing alcohol-related risks among community populations of SMW who may view their heavy drinking as normative and not in need of change due to the visibility of alcohol consumption in LBQ community spaces. Thus, the present intervention strategy diverges from and is intended to complement more intensive intervention programs being developed to meet the needs of heavy drinking SMW already motivated to reduce their consumption (e.g., Bush et al., 2019) and those seeking culturally tailored treatment for AUD and comorbid mental health problems (e.g., Pachankis et al., 2020). 


\section{CHAPTER 1}

\section{Literature Review}

\subsection{Sexual Orientation-based Alcohol Use Disparities}

Relative to women who identify as heterosexual, experience only opposite-sex attractions, and only have sex with men, research has documented a greater likelihood of heavy drinking, negative-alcohol-related consequences, and alcohol dependence among sexual minority women (SMW; Hughes, 2011; Hughes et al., 2020), a category which includes women who psychologically identify as lesbian, bisexual, pansexual, or queer in addition to those who report having sex with women and/or experiencing same-sex attraction (Sell, 1997; Talley et al., 2012). To date, most research into sexual orientation-based alcohol use disparities has focused on the sexual identity dimension of sexual orientation, comparing the consumption patterns and alcohol-related experiences of women identifying as lesbian and bisexual to those identifying as heterosexual (Hughes et al., 2020). For instance, findings indicate that compared to heterosexual women of the same age, lesbian and bisexual identified women are more than twice as likely to engage in heavy drinking (Fish, 2019; McCabe, Hughes, Bostwick, West, \& Boyd, 2009), 4-7 times more likely to meet DSM criteria for alcohol dependence, and 9-11 times more likely to report that their drinking has led to serious interpersonal consequences (Drabble \& Trocki, 2005; McCabe et al., 2009). Additionally, while heterosexual women tend to adopt safer alcohol use habits as they age, lesbian and bisexual women are more likely to continue patterns of heavy drinking into adulthood (Burgard, Cochran, \& Mays, 2005; McCabe et al., 2009; Rosario et al., 2014) increasing their risk for several cancers (Fredriksen-Goldsen, Hoy-Ellis, \& Brown, 2015; Rosario et al., 2014) and cardiovascular disease (Caceres et al., 2017; Farmer, Jabson, Bucholz, \& Bowen, 2013). In fact, heavy drinking is recognized as a syndemic factor in this population, 
interacting with other health-risk behaviors (e.g., smoking, sedentary behavior) to increase medical morbidity and healthcare costs (Coulter, Kinsky, Herrick, Stall, \& Bauermeister, 2015; Valentine et al., 2015).

Despite their elevated levels of consumption and alcohol-related risks, targeted evidencebased alcohol interventions for SMW have been slow to emerge (Blume, 2016; Hughes et al. 2020, Talley, 2013) and research investigating alcohol treatment utilization in this population remains limited. In sum, findings suggest that SMW utilize treatment programs and services at similar rates as do their sexual majority peers despite the former group's substantially greater likelihood of alcohol-related problems and dependence (Allen \& Mowbray, 2016; Grella, Greenwell, Mays, \& Cochran, 2009; McCabe et al., 2013). Further, among women who do access treatment, findings suggest that SMW may be more likely than their heterosexual peers to present with extreme patterns of consumption and severe health conditions (Cochran \& Cauce, 2006; Lipsky et al., 2012). Thus, problem drinking SMW may delay treatment for some time, a factor that may complicate their eventual recovery efforts.

\subsection{Explanations and Implications for Intervention}

\section{Sexual Minority Stress \& Stigma}

The dominant perspective for understanding poorer mental/physical health and increased health-risk behaviors among sexual minorities is that of sexual minority stress (Hatzenbuehler, 2009; Lick et al., 2013; Meyer, 2003). Minority stress refers to the unique, chronic, and socially constructed stress that members of marginalized groups experience due to the prejudice and discrimination they experience in society. Meyer (2003) differentiates between two specific types of minority stressors that negatively impact mental and physical health among lesbian, gay, and bisexual (LGB) populations. Distal sexual minority stressors are external to the individual, 
operate at the interpersonal level, and include experiences of prejudice, rejection, harassment, discrimination, and violence rooted in a heterosexist society. In contrast, proximal sexual minority stressors originate within the individual, often as a function of, or in reaction to, external stressors, and include experiences such as internalization of stigma, sexual identity concealment, and anticipated rejection. Thus, this theoretical perspective emphasizes the role that possessing a disadvantaged, stigmatized sexual minority identity plays in SMW's alcohol consumption, explaining that increased drinking and dependence among SMW may derive from separate and combined effects of distal and proximal sexual minority stressors.

Indeed, a growing literature informed by this model has established direct associations between SMW's alcohol-related experiences and exposure to distal and proximal stressors. Internalized stigma has been the most frequently examined proximal stressor among SMW (Hughes et al., 2020), with numerous studies revealing positive cross-sectional relationships between stigma internalization, heavy drinking, and alcohol-related problems among lesbian and bisexual women (e.g., Amadio, 2006; Kalb, Gillis, \& Goldstein, 2018; Lehavot \& Simoni, 2011; Weber, 2008). Survey studies with SMW have also documented positive relationships between alcohol-related outcomes and exposure to distal stressors taking the forms of sexual orientationbased discrimination (e.g., Lewis, Winstead, Lau-Barraco, \& Mason, 2017; Slater, Godette, Huang, Ruan, Kerridge, 2017), bullying (e.g., Polliet, Mallory, \& Fish, 2018), verbal/physical attacks (e.g., Mereish, O’Cleirigh \& Bradford, 2014; Phillips et al., 2020), and microaggressions (e.g., Scharer \& Taylor, 2017). However, longitudinal relationships between sexual minority stress experiences and alcohol-related outcomes remain understudied and less clear. For example, in one of the only prospective studies to assess alcohol outcomes in relation to total exposure to numerous distal and proximal stressors among SMW, sexual minority stress 
exposure predicted negative alcohol-related consequences one year later; but did not significantly predict frequency nor quantity of alcohol consumed (Wilson et al., 2016).

Expanding Meyer's original minority stress model, Hatzenbuehler's (2009) psychological mediator framework differentiates between group-specific sexual minority stress experiences, which include the distal and proximal stressors described by Meyer (2003), and general psychological processes that occur among all individuals regardless of sexual orientation. This framework identifies several general social/interpersonal, emotional, and cognitive processes by which sexual minority stress experiences may diminish well-being. For example, Hatzenbuehler's social/interpersonal pathway posits that sexual minority stress experiences can negatively impact well-being through increased isolation, loneliness, and diminished social supports. Stressors can also diminish mental and physical health through emotional and cognitive processes including dysregulation, rumination, maladaptive coping, negative self-schemas, and shame. Although longitudinal research is needed, several cross-sectional studies with SMW support the mediational roles of these constructs in the relationship between minority stress exposure and alcohol use (e.g., Fitzpatrick, Dworkin, Zimmerman, Javorka, \& Kaysen, 2020; Lewis, Mason, Winstead, Gaskins, \& Irons, 2016; Rogers et al., 2017).

In addition to the interpersonal- and individual level forms of stigma described by the minority stress model, structural stigma refers to the overarching societal-level conditions, cultural norms, and institutional policies that constrain the opportunities, resources, and wellbeing of the stigmatized (Hatzenbuehler, 2014; Hatzenbuehler, 2016; Hatzenbuehler \& Link, 2014; Hatzenbuehler, Jun, Corliss, \& Austin, 2015). Operationalized in terms of place-specific discriminatory legislation, anti-gay public opinion, a low population density of same-sex couples, and an absence of supportive LGBTQ community resources, recent studies have 
examined structural stigma in relation to mental and physical health outcomes among samples of LGB youth as well as substance use among sexual minority men (for a review see Hatzenbuehler, 2016). In sum, findings suggest that sexual minority people who reside in communities with greater structural stigma have higher rates of adverse mental and physical health outcomes than do sexual minority people who reside in communities with lower levels of structural stigma (Hatzenbuehler, 2014; Hatzenbuehler, 2016; Hatzenbuehler \& Keyes, 2013). Structural stigma has also been theorized to uniquely contribute to SMW's disproportionate drinking. However, to date, only a single published study has examined the legislative dimension of structural stigma in relation to alcohol use and negative alcohol-related consequences among SMW. Everett, Hatzenbuehler, and Hughes (2016) compared data collected from SMW residing in Illinois conducted before and after the state's passage of legislature legalizing same-sex unions. Participants interviewed before the passage of this legislation reported more adverse consequences due to alcohol consumption than did those interviewed after this legislation was passed and enacted. This finding suggests that the legislative component of structural minority stigma may be an antecedent to alcohol-related negative consequences among SMW and encourages additional research to thoroughly investigate structural stigma in the context of alcohol-related risks in this population.

\section{Intervention Strategies Informed by Sexual Minority Stress and Stigma Research}

The Transactional Model of Stress (Folkman \& Lazarus, 1984; Folkman et al., 1991)

outlines two strategies by which stress effects rooted in stigma experienced at individual, interpersonal and structural levels can be alleviated. The first strategy targets the existence of stigma itself. That is, interventionists seeking to reduce drinking among SMW might develop programs that seek to reduce the degree to which SMW internalize stigma and view the self 
negatively, implement family, school, or workplace-based programs in order to reduce the frequency with which SMW experience rejection, exclusion, and violence due to their sexual minority status, or enact new legislature or social policies to extend additional resources and rights to SMW. To date, however, efforts to reduce heavy alcohol use among SMW through such strategies remain scarce (Blume, 2016; Hughes et al., 2020). Although several pilot interventions have shown promise in lessening negative attitudes toward LGBTQ individuals (e.g., RamirezValles, Kuhns, Manjarrez, 2014), increasing positive self-schemas among sexual minorities (e.g., Riggle, Gonzalez, Rostosky \& Black, 2014), and bolstering LGBTQ community resources (Ratts et al., 2013), no published studies have examined alcohol-related outcomes among SMW (Chaudoir, Wang, \& Pachankis, 2015).

As an alternative to intervening to reduce stress itself, the second strategy seeks to mitigate the negative impacts of stress by bolstering individuals' personal resources for adaptive coping. These types of interventions commonly take the form of intensive, sexual identity affirming, cognitive-behavioral programs that seek to reduce health-risk behaviors by bolstering individuals' coping resources and understanding of stigma-related processes (Chaudoir et al., 2015). Several interventions of this type have shown promise in reducing substance use and sexual health-risk behaviors among sexual minority men (e.g., Panchankis et al., 2019; Parsons et al., 2017). Extending this work to SMW, an intensive, coping-focused cognitive-behavioral intervention is currently being developed to address heavy drinking among lesbian and bisexual women presenting with comorbid depression and anxiety (Pachankis et al., 2020). However, these types of interventions are costly, resource-intensive, and thus, may be challenging to scale. Preliminary research also suggests that their effectiveness may be concentrated among individuals who internalize sexual minority stigma and view their health-risk behaviors and 
mental health issues as consequences of such stigma (Millar, Wang, \& Pachankis, 2016). Thus, although these types of interventions may hold future promise for stigma-internalizing SMW already motivated to seek help for their problematic drinking, they may be unlikely to engage the larger population of SMW who do not view their heavy drinking as problematic and lack the motivation to change their behavior. Barriers to program implementation and engagement are likely to be rooted in the secondary explanation for SMW's excessive drinking: the centrality of alcohol use in queer socialization contexts.

\section{Queer Socialization \& Social Norms}

SMWs' disproportionate alcohol use has also been explained in terms of the culture of drinking ingrained within lesbian, bisexual, and queer communities, wherein alcohol-serving venues serve as points of community entry and primary environments for socialization (Cogger, Conover, \& Israel, 2012; Drabble \& Trocki, 2014; Gruskin, Byrne, Kools, \& Altschuler, 2007; Parks, 1999). Indeed, nationally representative survey data demonstrate that SMW attend bars, nightclubs, and parties more frequently and consume more alcohol in these contexts than do their heterosexual peers (Trocki, Drabble \& Midanik, 2005). Qualitative accounts from SMW suggest that the position of bars and nightclubs as center-points for queer socialization may lead young SMW to view heavy drinking as a rite of passage or requisite social lubricant for interacting and initiating relationships with fellow SMW (Gruskin et al., 2007; Parks, 1999). Research also suggests that SMW perceive heavy drinking to be more typical among fellow lesbian and bisexual women relative to heterosexual women with elevated perceptions of drinking among SMW predicting their own levels of consumption (Litt, Lewis, Rhew, Hodge, \& Kaysen, 2015).

Other findings suggest that the high visibility and salience of heavy drinking in lesbian and queer community spaces may lead to long-lasting, positive associations between sexual minority 
identity and alcohol consumption. For example, among lesbian-identified SMW, findings from two survey studies indicate that lesbian bar-based drinking contexts during the early stages of lesbian identity development (i.e., coming out, forming initial relationships) can have a lasting impact on alcohol use trajectories--- even predicting lesbians' drinking into middle adulthood, after their bar-going has waned (Parks \& Heller, 2013; Parks, Hughes, \& Kinnison, 2007).

Social norms theory (SNT; Berkowitz, 2004; Perkins, 2003; Perkins \& Berkowitz, 1986) provides a useful framework for understanding the mechanisms by which current and past socialization in LGB community bars and nightclubs might lead to heavier drinking and greater alcohol-related risks among SMW long after they physically leave the bar. Central to this theory is the distinction between actual and perceived norms. Whereas actual norms reflect the behavioral and attitudinal reality among members of a group, perceived norms constitute group members' perceptions of normative behaviors and attitudes within the group. SNT emphasizes that people are prone to inaccuracies when estimating "typical" behaviors and attitudes among members of their peer group. Specifically, people tend to overestimate the degree to which peers approve of, and engage in, problematic and risky behaviors like heavy drinking, smoking, and drug use. At the same time, people are prone to underestimating the degree to which peers engage in prosocial and healthy behaviors and hold positive attitudes toward these activities (Berkowitz, 2004; Perkins, 2003; Perkins \& Berkowitz, 1986).

In the domain of alcohol use, research has established that an individual's perceptions of drinking in their peer group to be among the most reliable and robust predictors of their own future alcohol use (Berkowitz, 2004, Miller \& Prentice, 2016, Perkins, 2003) and SNT references two specific types of perceptual biases that illustrate ways in which over-estimations of peer drinking norms can work to influence the perceiver's alcohol consumption. The first, pluralistic 
ignorance, refers to the false assumption that the attitudes or behaviors of relevant peers are different from one's own, when in fact, they are similar (Miller \& McFarland, 1991; Prentice \& Miller, 1993). This type of misperception often results in a person changing his or her own behaviors or attitudes in the direction of the misperceived group norm. As theorized in SNT, individuals who abstain or infrequently drink may falsely view their behavior as different from most of their peers (Miller \& Prentice, 2016). This false perception of deviance, in turn, can result in the individual initiating or increasing their drinking in order to approximate the behavioral standard they misperceive to be normative in the group (Berkowitz, 2004; Perkins, 2003). The second common form of misperception, false consensus, refers to the incorrect belief that others' behaviors and attitudes are similar to one's own when, in reality, they are not (Ross, Green, \& House, 1977). In the SNT alcohol intervention literature, false consensus is theorized to act as a barrier to behavior change among heavy drinkers by functioning to maintain an individual's perception that his/her behavior is aligned with the group norm and, therefore, is not problematic, unusual, or a significant risk to their health (Berkowitz, 2004; Miller \& Prentice, 2016; Perkins, 2003).

Most germane to the issue of bar-based socialization among SMW is the tenet of SNT, which illuminates sources of normative misperception. This theory positions observable public behavior as the most influential source of information fueling misperceptions of peer drinking norms (Berkowitz, 2004; Perkins, 2003). That is, over-estimations of how much and how often peers drink are most likely to result from observing a minority of the peer group publicly engaging in high-risk alcohol use, as would be likely to occur among SMW in LGB bar, club, and party settings. Observations of heavy drinking are remembered to a greater degree than responsible and more mundane behaviors that may be more common in the group but are less 
visible or memorable (Berkowitz, 2004; Miller \& Prentice, 2016; i.e., alcohol abstinence in bars, non-alcohol-related activities taking place outside of these community spaces). Thus, because LGB bars, clubs, and parties remain the most visible and accessible physical spaces for SMW to identity sexual minority peers, observe the behaviors of like-identified women and meet potential friends and lovers, the alcohol inundated observations that occur in these social settings may make SMW particularly likely to overestimate how much and how often other SMW consume alcohol, and in turn, adjust their drinking to approximate this misperceived, risky normative standard.

Consistent with these predictions, several cross-sectional studies have documented lesbian and bisexual women's' tendencies to overestimate sexual identity specific descriptive drinking norms, with strong cross-sectional relationships observed between perceived drinking norms and alcohol consumption (Boyle, Kettering, Young, \& LaBrie, 2020; Boyle, LaBrie, \& Witkovic, 2016; Ehlke, Stamates, Kelley, \& Braitman, 2019). Further, in one study with lesbians (Boyle et al., 2016), greater frequency of lesbian bar patronage was significantly associated with elevated peer drinking norms after controlling for participants own drinking, suggesting that the observations that take place in these settings do play a role in lesbians' normative misperceptions around drinking. In addition, Litt and colleagues (2015) followed a large sample of young SMW over a 3-year period and assessed perceived norms for the typical "sexual minority woman" and participants' own alcohol consumption each year. Strong relationships between perceived drinking norms and alcohol consumption were observed at each assessment, and after accounting for cross-sectional relationships, perceived sexual minority drinking norms predicted consumption one year later. In sum, although only a handful of norm-focused studies have been conducted with this population to date, relationships between perceived drinking norms and 
alcohol consumption among SMW are clear and highly consistent with those observed among populations of college students (Dotson, Dunn, \& Bowers, 2015), military personnel (Neighbors et al., 2014), and working adults (Walters \& Woodall, 2003) commonly targeted by brief social norms alcohol interventions. Moreover, in addition to the general frequency and quantity alcohol use norms typically of focus in SNT-based research in other populations, research suggests that SMW also tend to overestimate descriptive norms specific to coping-motivated drinking following collectively experienced stigmatizing events such as the Pulse Nightclub shooting (Boyle et al., 2016) and 2016 U.S. presidential election (Boyle et al., 2019), with these misperceptions also contributing to current and future drinking beyond their stress impact.

\section{Interventions Designed to Correct Misperceived Norms}

The most widely studied and efficacious alcohol intervention strategy informed by SNT is that of personalized normative feedback (PNF; Miller \& Prentice, 2016). Designed to correct over-estimated peer drinking norms, PNF alcohol interventions first prompt members of a target group to privately answer a series of survey questions about their perceptions of group member's alcohol use and their own drinking (Berkowitz, 2004; Perkins, 2003; Perkins \& Berkowitz, 1986). Specifically, questions about perceived descriptive drinking norms in the group prompt participants to estimate the frequency and quantity of alcohol consumed by the "typical" group member, or the proportion of group members who have engaged in a specific high-risk drinking behavior (e.g., having $8+$ drinks in one night) or have had a particular intoxication-related experience (e.g., blacking out). Actual descriptive norms for alcohol use behaviors within the group are then derived from subsequent survey questions, which prompt participants to report on their own parallel drinking behaviors and alcohol-related experiences. Following this data collection step, PNF is delivered to participants in the form of an individualized graphical report 
which highlights discrepancies between their perceptions of group members' drinking, group members' actual drinking, and their own drinking. This feedback is designed to change the individuals' perceptions of "normal" drinking among group members by exposing their misperceptions of the actual group norm as well as by comparing their own drinking behavior to that actually normative within the group (Berkowitz, 2004; Miller \& Prentice, 2016; Perkins \& Berkowitz, 1986). To date, this simple and cost-effective approach has been successful in reducing alcohol use in community samples of adolescents (Doumas, Esp, Turrisi, Hausheer \& Cuffee, 2014), college students (Borsari \& Carey, 2003; Dotson, Dunn, \& Bowers, 2015), military veterans (Pedersen et al., 2017), active duty service members (Pemberton et al., 2011), and working adults (Hester, Squires, and Delaney, 2005), with meta-analyses indicating an average effect size of $d=.22$ (Riper et al., 2009) relative to control PNF on non-health related topics.

\subsection{Examining the Utility of PNF for SMW: Unanswered Questions}

Research has yet to evaluate the efficacy of correcting peer drinking norms to reduce alcohol use among SMW. However, supporting the promise of PNF for this population, in university and military samples this strategy has been found particularly effective in reducing alcohol consumption among women (Murphy et al., 2004; Saunders, Kypri, Walters, Laforge, \& Larimer, 2004), individuals for whom the reference peer group or community is important to their overall sense of self (Neighbors et al., 2010), those reporting social (Neighbors et al., 2004) and/or coping (Young et al., 2016; Young et al., 2018) motivations for drinking, and heavy drinkers not yet aware that their consumption exceeds normative standards (Bertholet et al., 2016). As PNF alcohol interventions have not yet been evaluated among sexual minorities (nor 
other stigmatized populations), several critical questions about PNF intervention content, efficacy, and potential moderators in this population remain to be answered.

\section{For SMW, what is the appropriate level of specificity for PNF reference groups?}

In the PNF literature, reference group specificity refers to the amount of demographic information (e.g., age, sex, race, ethnicity, sexual identity, etc.) provided about the "typical peer" on which questions about alcohol use are based (Borsari \& Carey, 2003; Larimer et al., 2009). In general, higher reference group specificity increases the degree to which the peers in question are similar and meaningful to the perceiver, thereby strengthening the relationship between perceived norms and behavior (e.g., Larimer et al., 2009; Liu et al., 2017). However, individuals are unlikely to misperceive norms for groups too high in specificity (i.e., typical peer of the same age, race, ethnicity, sexual identity, and level of education living in the same town) as groups are too directly observable (LaBrie et al., 2013; Miller \& Prentice, 2016). Highly specific reference groups also make it difficult to deliver meaningful, stable actual norms to participants in PNF as the sizes of sub-group cells on which actual norms are derived become too small and unstable (Pedersen et al., 2016). As such, reference groups in successful PNF interventions are moderate in specificity, typically sharing two key demographic characteristics or social categories with the participant (LaBrie et al., 2013). Further, these characteristics/categories tend to map onto important and accessible aspects of self and simultaneously reflect meaningful differences in target behaviors/attitudes. For instance, in a recent PNF intervention for young veterans of the U.S. military, reference groups were specific to participants' sex and veteran status (Pedersen et al., 2016; Pedersen et al., 2017). For this population, sex is both psychologically relevant and distinguishing in terms of patterns of drinking as men tend to consume greater quantities of 
alcohol than women. The second category, status as a veteran of the U.S. military, represents an important group membership for social identification.

Together, this reference group logic and prior research with SMW offer guidance as to the social categories and characteristics that might comprise efficacious PNF reference groups for SMW. For instance, relative to sexual behavior and attraction, sexual identity is both the dimension of sexual orientation most reliably associated with increased alcohol-related risks among SMW (Coulter, Marzell, Saltz, Stall, \& Mair, 2016; Drabble, Midanik, \& Trocki, 2005; Hughes et al., 2020) and most likely to compose a meaningful and salient aspect of one's overall sense of self (Cox \& Gallois, 1996; Sell, 1997). Several studies have also documented differences in alcohol consumption by sexual identity, with heavier drinking and greater symptoms of dependence observed among bisexual women relative to lesbians (e.g., Lewis et al., 2019; Schuler \& Collins, 2020). Moreover, the stigmatization of bisexuality within lesbian communities (Feinstein, Dyar \& London, 2017; Hartman, 2008; Matsick \& Rubin, 2018) and other sources of intra-community tension documented among lesbian, bisexual, and queeridentified SMW (Sexton, Flores, \& Bauermeister, 2018) further underscore the appropriateness of assessing perceptions of sexual identity specific norms rather than combined norms for the “typical sexual minority woman”, which may be less salient and meaningful to lesbian, bisexual, and queer women alike.

In terms of self-relevance and alcohol-related risks, other potentially appropriate categories for reference group specificity among SMW include race, ethnicity, and age. At present, findings regarding relationships between race, ethnicity, and heavy drinking and alcohol dependence among SMW remain mixed and inconclusive (for a review, see Hughes et al., 2020). Further, although race and ethnicity undoubtedly represent salient and important aspects of self, 
correcting norms for sub-groups based on these variables in addition to sexual identity may lead to poor estimates of actual norms based on very small numbers of participants for some racial and ethnic minority SMW (e.g., typical American Indian or Alaska Native bisexual woman). In contrast to race/ethnicity, prior work has revealed reliable differences in patterns of alcohol consumption by age, with younger SMW more likely to engage in heavy drinking and report symptoms of dependence than older SMW (Austin \& Irwin, 2010; Burgard, Cochran, \& Mays, 2005; Hughes et al., 2006). These age-related differences in consumption suggest that delivering actual drinking norms predominantly derived from SMW in their 20s to SMW in their 40s and 50s may be more likely to enhance than reduce alcohol-related risks. Conversely, actual norms including data from SMW in their 40s and 50s may be less relevant, and therefore, less likely to motivate behavior change among younger SMW. Thus, to ensure risk-reduction and selfrelevance, the current study tests the efficacy of a PNF intervention which asks questions and delivers actual drinking norms specific to the participants' sexual identity and age-group (i.e., the typical [lesbian/bisexual/queer woman] in her [20s, 30s, 40s, 50s+]).

\section{Is it beneficial to correct coping norms in addition to drinking norms among SMW?}

Previous research has linked heavy drinking among SMW to stronger social and coping motives for alcohol use (e.g., Dworkin, Cadigan, Hughes, Lee, \& Kaysen, 2018; Hughes et al., 2020). These same drinking motives are also common among university and military populations, and notably, PNF interventions designed to correct norms for peers' frequency and quantity of alcohol consumption have been found to be highly effective among participants reporting coping motives (Neighbors et al., 2004; Young et al., 2016; Young et al., 2018), suggesting the appropriateness of alcohol PNF for SMW. However, in contrast to more general coping with negative affect in non-stigmatized college and military populations, the sexual 
minority stress literature suggests that SMW more specifically drink to cope with the stigmatizing experiences that follow from possessing a devalued sexual minority identity (Lehavot et al., 2011; Meyer, 2003). Following from this work, subsequent research inspired by SNT has also established that sexual minorities misperceive the behaviors sexual minority peers' engage in to cope with stigmatizing events (Boyle et al., 2016; Boyle et al., 2017), overestimating peers' coping-motivated use of alcohol and other drugs in the aftermath of such events, while simultaneously underestimating the proportion of sexual minority peers who engage in adaptive, healthy, adaptive coping behaviors (social support seeking, mediation, etc.). Thus, the second aim of the present research is to evaluate whether the drinking reduction effects associated with alcohol PNF can be enhanced by also correcting misperceived norms for the coping behaviors enacted by sexual identity and age-specific peers experiencing sexual minority stigma.

\section{Does sexual minority stigma diminish or enhance PNF intervention efficacy among SMW?}

To date, very little research has simultaneously examined perceptions of sexual identityspecific drinking norms, sexual minority stressors, or stigma-related variables as predictors of SMW's drinking or considered how these predictors may influence or interact with one another over time. However, sexual minority stress and stigma have long been positioned in the literature as primary targets for substance use interventions and several review papers implicitly convey the perspective that social disadvantage and stigma-related stress may render mainstream intervention strategies efficacious in other populations ineffective among sexual minorities (e.g., Green \& Feinstein, 2012; Hughes et al., 2020; Talley, 2013). Although sparse and not specific to SMW, PNF, nor alcohol use, some findings with other stigmatized populations support this view. For instance, psychosocial sexual health intervention programs have been found to be less 
effective in changing behavior among racial minority participants residing in states with greater race-based structural stigma (Reid, Dovidio, Ballester, \& Johnson, 2014), which the researchers attributed to participants' stigma-based mistrust of outsiders and diminished confidence in the health domain. Other sexual health and HIV prevention research similarly suggests that interpersonal and structural forms of stigma may present barriers to individual-level intervention engagement, intervention adherence, and study retention among sexual and racial minority men (Oldenburg et al., 2015). These findings suggest that the efficacy of a PNF alcohol intervention may be undermined among SMW exposed to greater interpersonal and structural stigma as a function of their sexual minority status.

However, two longstanding social psychological theories suggest the opposite- that is, correcting over-estimated drinking and coping norms may be most effective among SMW with greater exposure to these forms of sexual minority stigma because these women will have an enhanced motivation to conform to salient group norms once corrected. For instance, selfcategorization theory (Hogg \& Reid, 2006; Turner, Hogg, Oakes, Reicher, \& Wetherell, 1987; Turner \& Reynolds, 2011) contends that when inter-group threat is experienced in a self-relevant domain, as would be the case when a SMW experiences discrimination, harassment, or violence due to her sexual minority status, she will be particularly likely to self-stereotype, turning to salient and accessible ingroup norms to guide to her behavior. Similarly, in the context of severe forms of interpersonal stigma such as violence and harassment based on sexual minority status, terror management theory posits that living up to the perceived cultural standards (Greenberg, Pyszczynski, \& Solomon, 1986; Greenberg, Solomon, \& Pyszczynski, 1997) and salient norms (Jonas \& Fritsche, 2012; Jonas et al., 2008) of a self-relevant group can protect against the deeply rooted fears of mortality likely to arise from such experiences. Thus, following from both 
theories, to the extent that a SMW experiences prejudice or victimization due her sexual identity and has inflated perceptions of how much same-identity peers drink, she may be especially likely to increase her drinking as a means of conforming to this inflated normative standard. In turn, PNF designed to correct over-estimated sexual minority-specific peer norms for general alcohol consumption and unhealthy stigma-coping behaviors may allow SMW who are motivated to conform to salient group norms as a function of their experiences with interpersonal and structural stigma to align their behavior to risk-reducing "true" group norms.

Such an interplay between sexual minority stigma and perceived group norms are suggested by findings from two cross-sectional survey studies, the first focused on gay men and the second focused on lesbian and bisexual women. First, Hamilton and Mahalik (2009) assessed gay men's perceptions of peer norms for several health-risk behaviors (i.e., use of alcohol, tobacco, illicit drugs, and engaging in risky sexual practices) and examined the degree to which these perceived norms, current and historical exposure to sexual minority specific prejudice and discrimination, and other psychosocial factors individually predicted, and interacted to predict, participants' own health-risk behaviors. Results revealed that perceived norms were individually the strongest predictor of health-risk behaviors and, importantly, this relationship was moderated by current and historical exposure to prejudice and discrimination such that a stronger relationship between perceived norms and participants' own behaviors was observed when exposure to sexual minority-specific prejudice and discrimination was high relative to low. These findings provide the first evidence that sexual minority stigma experiences may be associated with self-stereotyping and conformity to perceived sexual minority group norms.

In a more recent study, Lewis and colleagues (2017) examined the motivational mechanisms by which sexual identity-based discrimination and stigma-consciousness impact 
drinking among lesbian and bisexual young women. Although perceptions of drinking norms were not directly assessed, the researchers did assess a related construct, conformity drinking motives, which measure the motivation to drink in order to align one's drinking to the perceived normative standards of a peer group (Cooper, 1994; example item, I drink in order to fit in with a group I like). Grounded in sexual minority stress research and theory (Hatzenbuehler, 2009; Meyer, 2003), the researchers had anticipated that positive relationships between stigma variables and alcohol consumption would be explained by coping drinking motives. However, results revealed that this relationship was mediated by both conformity and coping drinking motives. That is, greater sexual minority-based discrimination and stigma-consciousness were associated with greater coping and conformity motives for drinking, which, in turn, explained the positive relationship between sexual minority stigma variables and alcohol consumption. These findings suggest that one pathway by which sexual minority stigma may impact drinking among SMW is through increased motivation to conform to the perceived norms of a self-relevant group.

Taken together, findings from these studies suggest that conformity to salient sexual minority group norms may be particularly likely when prejudice and discrimination are experienced by SMW. Thus, to the extent that sexual minority peer drinking and maladaptive stigma-coping norms are over-estimated, salient, and accessible, the efficacy of PNF correcting these norms may be enhanced, rather than diminished, among stigma burdened SMW. The present research seeks to directly test whether greater interpersonal and/or structural stigma exposure strengthen the relationship between perceived sexual identity-specific norms and alcohol consumption (i.e., increasing conformity), thereby making a brief PNF intervention 
designed to reduce alcohol use more, rather than less, effective among SMW with greater exposure to these forms of sexual minority stigma.

\section{Are there other participant-level factors that enhance/diminish PNF efficacy among SMW?}

PNF intervention research with university and military populations supports the classification of PNF as a universal, brief prevention and intervention strategy with broad utility for drinkers regardless of their individual characteristics (Dotson et al., 2015;Walters \& Neighbors, 2005). However, one moderator of intervention efficacy that has been consistently identified by researchers is pre-intervention level of alcohol consumption. Relative to lighter drinkers, heavier drinkers naturally have greater room for behavior change following PNF's correction of drinking norms (Miller \& Prentice, 2016, Pedersen et al., 2017). As a result, in studies that recruit participants at all levels of alcohol consumption, drinking level reliably moderates PNF intervention efficacy, with larger reductions in drinking observed among participants engaging in heavier consumption pre-intervention. Thus, to the extent that SMW taking part in a PNF intervention exhibit sufficient group-level heterogeneity in their preintervention drinking levels, heavier drinking SMW would be expected to exhibit larger reductions in their drinking post-PNF relative to their lighter drinking peers who have less room for change.

In addition to pre-intervention level of alcohol consumption, other potential moderators of PNF intervention efficacy among SMW include the social categories and demographic characteristics both reflected in (i.e., sexual identity, age) and absent from (race/ethnicity, relationship status) from the specificity of reference groups. Although results have been mixed for race and ethnicity, previous research has found heavy drinking to be more likely among SMW who are single (relative to coupled; Veldhuis et al., 2019), bisexual (relative to lesbian; 
Lewis et al., 2019; Schuler \& Collins, 2020), and younger (relative to older; Austin \& Irwin, 2010; Burgard et al., 2005). However, to date, no published studies have compared the relative sizes of discrepancies between perceived and actual norms or the strengths of relationships between perceived norms and alcohol consumption among sub-groups of SMW based on these characteristics; thus, it is unclear whether they are likely to moderate PNF intervention efficacy. Further, in PNF intervention studies with university and military populations, these characteristics have either not been examined as moderators (i.e., sexual identity, relationship status) or not been found to moderate efficacy (i.e., race/ethnicity, age; Pedersen et al., 2017, LaBrie et al., 2013). Given the gaps in norms-focused research with SMW and lack of findings suggesting that PNF interventions are more or less effective in other populations as a function of participants' sexual identity, age, race/ethnicity, or relationship status, these potential moderators will be examined through exploratory analyses.

\section{Summary of Efficacy Aims}

The present research aims to address gaps in the literature related to PNF intervention efficacy among SMW. Specifically, this research will evaluate: 1) whether delivering PNF to correct sexual identity and age-specific peer drinking norms reduces alcohol use relative to control PNF; 2) whether delivering PNF to also correct stigma-coping norms reduces drinking better than PNF on alcohol use alone; 3) whether interpersonal or structural forms of stigma exposure enhance or diminish the efficacy of PNF alcohol use and/or stigma-coping among SMW; and, 4) whether treatment PNF on drinking and stigma-coping are differentially effective among SMW as a function of pre-intervention level of alcohol consumption, sexual identity, age, race/ethnicity and/or relationship status. 
Importantly, the ability to test these aims rests on the successful development and implementation of a PNF intervention able to attract, engage, and retain a large, diverse sample of light, moderate, and heavy drinking SMW. The next chapter describes the methodological limitations and implementation challenges that have worked against traditional PNF interventions and efforts to evaluate these programs in other populations, highlights points of innovation designed to remedy these issues among SMW, details supporting research, and fully introduces the current hybrid efficacy/feasibility trial. 


\section{CHAPTER 2}

\section{Intervention Development}

\subsection{PNF Intervention Modalities, Limitations, and Challenges}

Standalone PNF interventions initially took the form of paper-based surveys and feedback reports delivered to college students via postal mail (e.g., Collins, Carey, \& Smyth, 2005). Later, these interventions were delivered onsite via software programs installed on campus computers with a university researcher or administrator present (see Dotson et al., 2015, for a review). More recently, to increase cost-effectiveness and scalability, remotely delivered, web-based PNF intervention formats emerged to take advantage of widespread internet and email use (e.g., LaBrie et al., 2013; Lewis \& Neighbors, 2015). Today, populations of college students, military personnel, and working adults are most commonly invited to take part in remotely delivered web-based PNF interventions by an email from their institution (i.e., university, military base, employer) with participation either mandatory or incentivized (LaBrie et al., 2013; Dotson et al., 2015; Bedendo, Ferri, de Souza, Andrade, \& Noto, 2019). In these interventions, PNF is delivered immediately after participants answer alcohol-related survey questions with actual norms derived from previously collected, regional or institutional survey data (e.g., Borsari \& Carey, 2003; Neighbors et al., 2014). In the event that sufficient actual norm data for reference groups are not available, researchers typically recruit a separate sample of participants from the target population to take part in an incentivized norms documentation study prior to recruiting the intervention study sample (e.g., LaBrie et al., 2013; Pedersen et al., 2016). In either case, the text included in PNF clarifies the fact that actual norms are based on previously collected survey data and specifies the data source. 
Although web-based modalities increase the cost-effectiveness and scalability of PNF interventions, previous work also suggests drawbacks. For instance, research with college students suggests that web-based PNF leads to reliable but modest and relatively short-term reductions in drinking that fall short of the effects observed in PNF interventions delivered to students via onsite computers with a researcher or college administrator present (Dotson et al., 2015). Researchers have also identified a number of issues that, if remedied, could considerably increase the impact of web-based PNF. In particular, doubts about the credibility of actual drinking statistics presented from previously collected data sources (Hummer \& Davison, 2016; Miller \& Leffingwell, 2013), defensive reactions to feedback among heavy drinkers (Granfield, 2005; Steers et al., 2016), multi-tasking associated inattention, (Lewis \& Neighbors, 2015; Rodriguez et al., 2015), and low motivation among participants (Bedendo, Ferri, de Souza, Andrade, \& Noto, 2019) have been proposed as barriers to greater public health impact. The realworld suitability of this approach has also drawn criticism (Dempsey, McAlaney, \& Bewick, 2018) as researchers have struggled to implement web-based PNF interventions as well as engage and retain heavy drinkers outside of institutional settings where participation is mandatory or participants are offered compensation at the point of recruitment (Bedendo et al., 2019; Palfai, Winter, Lu, Rosenbloom, \& Saitz, 2014).

\subsection{PNF Intervention Development Considerations For SMW}

To date, few PNF interventions have been delivered to populations not attached to institutions or workplaces. However, Pedersen and colleagues found success in using targeted Facebook advertisements to recruit heavy drinking young military veterans into a brief PNF intervention (Pedersen et al., 2015; Pedersen et al., 2017), suggesting that social media-based advertisements may represent a fruitful strategy for reaching other hard-to-reach populations 
with PNF. As sexual minority adults have been found to use both the internet and mainstream social media platforms (e.g., Facebook, Instagram, Twitter) more than their heterosexual peers (Taylor, 2013; Seidenberg et al., 2017) and findings suggest that SMW have a preference for receiving health information online (Flanders et al., 2017; Park et al., 2018), a web-based PNF intervention advertised via social media channels may be appropriate for this population. This potential recruitment strategy is also supported by a recent pilot study which examined social media use in relation to alcohol use and perceptions of sexual identity specific drinking norms in a diverse sample of lesbian-identified women ranging in age from 18 to 40 years (Boyle, Kettering, Young, and LaBrie, 2020). Heavier use of mainstream social media platforms (i.e., Facebook, Instagram, Twitter) was significantly associated with heavier drinking, inflated perceptions of lesbian peer drinking norms, greater confidence in the accuracy of one's normative drinking (mis)perceptions, and greater interest in learning how one's own drinking compared to peers'. Thus, these findings indicate that the SMW most likely to benefit from a web-based PNF alcohol intervention may also be the most readily reached via popular social media sites.

However, previous research with SMW also suggests that social media-based recruitment efforts can be time-consuming and costly when large samples are needed and may lead to both data quality issues and problematic levels of attrition in longitudinal studies. For example, in the only longitudinal, web-based survey study to assess SMW's perceptions of drinking norms and alcohol consumption published to date (Litt et al., 2015), it took over a year for the researchers to recruit a large sample of lesbian and bisexual women from social media sites, bot-related data quality issues were observed despite targeted ads, and participant drop-out was substantial in follow-up surveys despite sizable monetary incentives for survey completion. Findings also 
suggest that SMW may be particularly difficult to attract and engage in health-related intervention efforts, regardless of recruitment strategy. For instance, in reviewing six community-based interventions targeting various health-risk behaviors among lesbian and bisexual women (e.g., unhealthy weight, sedentary behavior, smoking), Rizer et al. (2015) identified low responses rates, small sample sizes (e.g., 11 to 88 participants), and problems with attrition to be significant challenges to evaluation efforts. Concerns about attracting and engaging SMW are magnified in the PNF context, as this intervention strategy is most effective in reducing alcohol-related risks among individuals who do not view their drinking as excessive or see themselves as in need of intervention. To address these challenges, cultural tailoring and gamification are leveraged in a novel web-based PNF format in an effort to both engage SMW and minimize the attentional and motivational limitations associated with web-based PNF modalities in other populations.

\section{Increasing Relevance and Appeal Through Cultural Tailoring}

Cultural tailoring refers to "the development of interventions, strategies, messages, and materials to conform with specific cultural characteristics" (Pasick, D’Onofrio, \& Otero-Sabogal, 1996), and represents a common practice in adapting evidence-based health interventions for racial, ethnic, and sexual minority populations (Talley, 2013). In terms of specific cultural characteristics commonly targeted in tailoring efforts, social identity refers to the sense of self that people derive from their membership in a social group (Turner, Hogg, Oakes, Reicher, \& Wetherell, 1987). Tailoring often centers on understanding the salient and meaningful social identities in a target population and crafting intervention branding and content to be consistent with the values and norms of these social groups (Campbell \& Quintillani, 2006; Krueter \& Haughton, 2006). Notably, social identity is also an important construct in PNF interventions. 
That is, in traditional, untailored web-based PNF interventions for college students, larger reductions in drinking have been found among participants for whom featured reference groups were more self-relevant (e.g., Lewis \& Neighbors, 2015). Thus, for SMW, it may increase both intervention engagement and efficacy to strategically package a PNF intervention to attract and engage women for whom an LBQ community membership is an important aspect of self.

Related work has differentiated between surface structure and deep structure as two unique dimensions of cultural sensitivity to be addressed by researchers seeking to adapt existing interventions to improve health among members of disadvantaged minority groups (Resnicow, Baranowski, Ahluwalia, \& Braithwaite, 1999; Resnicow, Soler, Braithwaite, Ahluwalia, \& Butler, 2000). Consistent with the definition of cultural tailoring above, surface structure adaptations involve matching intervention materials and messages to align with the values and interests of the target group in order to ensure cultural fit. Surface structure adaptations also include the identification of the people, mediums, and environments that may be most appropriate and advantageous for promoting and delivering an intervention based on social identities and other cultural characteristics (Okamoto, Kulis, Marsiglia, Steiker, \& Dustman, 2014; Resnicow et al., 2000). In contrast, deep structure adaptations go beyond superficial modifications to more fully reflect within an intervention the social, historical, environmental, and psychological forces that influence well-being in the target group. Specifically, these deeper efforts involve infusing the unique cultural worldviews, beliefs, values, and behaviors of a population into an existing, evidence-based intervention in order to increase cultural relevance and maximize culture-specific resiliencies (Resnicow et al., 1999; Resnicow et al., 2000). The core components of an evidence-based intervention remain unchanged but the presentation and 
framing of intervention components are adapted to reflect culturally salient issues and themes

(Okamoto, Kulis, Marsiglia, Steiker, \& Dustman, 2014; Escobar \& Gorey, 2018).

Research has yet to directly compare the efficacy of culturally tailored versus nontailored alcohol interventions for SMW (Green \& Feinstein, 2012; Talley, 2013), and no studies conducted to date have directly investigated whether deep structure adaptations provide benefits beyond surface structure adaptations in tailored alcohol interventions for sexual minority populations (Bauer \& Wayne, 2005; Talley, 2013). However, these questions have been addressed in several comparative and meta-analytic studies with racial and ethnic minority groups. Relative to untailored (but otherwise matched) evidence-based interventions for these groups, culturally tailored versions have been found to significantly benefit recruitment, retention (e.g., Kumpher, Alvarado, Smith, \& Bellamy, 2002), and intervention efficacy (e.g., Barrera, Castro, Strycker, \& Toobert, 2013; Hall, Ibaraki, Huang, Marti, \& Stice, 2016). Further, supporting the prediction that deep structure cultural adaptations can work to maximize culturespecific resiliencies, Escobar and Gorey (2018) examined mental health intervention outcomes among Hispanic adults and found that tailored programs with deep structure adaptations (e.g., incorporating machismo and the importance of family as themes) were more efficacious than those which only included surface structure adaptations.

Most intervention cultural tailoring recommendations for SMW published to date have focused on surface structure adaptations. For example, health promotion researchers have recommended that intervention programs for SMW be culturally tailored to appeal to the social identities and values of LBQ women (Germanos, Deacon, \& Mooney-Somers, 2015; Mason \& Lewis, 2014; Sexton et al., 2018). Further, to increase the likelihood that SMW find programs to be responsive to their needs and judge programs to be credible, research suggests that 
intervention programs be led by ingroup peers sharing LBQ community memberships and either attached to, or supported by, LBQ community organizations likely to be familiar and trusted as sources of social and health information within these groups (Rizer et al., 2015). Building on this work and seeking to maximize cultural relevance, the current intervention approach incorporates surface structure recommendations and leverages insider knowledge of LBQ communities and the lived experiences of LBQ women to infuse several deep structure cultural themes into the framing and core components of a web-based PNF intervention. Specifically, this novel PNF intervention format seeks to incorporate LBQ women's' well-documented awareness of negative sexual identity-based stereotypes (e.g., Huxley, Clarke, \& Halliwell, 2014; Fingerhut \& Abdou, 2017; Gordon, 2006), desire for increased identity visibility (e.g., Fox \& Warber, 2015; Gabbay \& Wahler, 2002; Harerra, 2018) and enjoyment of intra-community competition and sport (e.g., Davis-Delano, 2014; Dolance, 2005; Mock, Misener, \& Havitz, 2019).

\section{Increasing Motivation and Engagement through Gamification}

The term gamification first emerged in American vernacular in 2008 to describe corporate efforts to boost business by integrating points and rewards into software intended to engage, retain, and reward loyal customers (Mangdalindan, 2010). Today, the term is more broadly defined as the application of digital game mechanics, aesthetics, and thinking to engage, motivate action, and promote learning in non-game contexts (Cugelman, 2013; Kapp, 2013; Kawachi, 2017, Seaborn \& Fels, 2015). In recent years, researchers seeking to motivate behavior change through computer and mobile app-based interventions have increasingly turned to popular digital game mechanics including game-framed narrative backstories, systems of points, chance elements, avatars, and leaderboards, as ways to increase intervention appeal, motivation, attention, and retention (for reviews see Floryan, Ritterband, \& Chow, 2019; Johnson et al., 
2016). To date, gamified digital health interventions including different constellations of these mechanics have shown promise in increasing physical activity (e.g., Edney et al., 2020; Hamri \& Maher, 2015), promoting self-management of chronic illness (e.g., Allam, Kostova, Nakamoto, \& Schulz, 2015; Kato, Cole, Bradlyn, \& Pollock, 2008), motivating smokers to quit (e.g., Blok et al., 2019; El-Hilly et al., 2016), and improving mental health (e.g., Fleming et al., 2017). Notably, gamification has also caught the attention of researchers seeking to engage sexual minority men in digital health promotion efforts; there are gamified digital interventions targeting mental health, sexual risk behaviors, and/or substance use currently in development or collecting feasibility data (e.g., Coulter et al., 2019; Flores-Aranda, Goyette, \& Larose-Osterrath, 2019; Hightow-Weidman, Muessig, Bauermeister, LeGrand, \& Fiellin, 2017; Mejia et al., 2017). Despite great enthusiasm around the prospect of gamification in the domain of digital health promotion efforts, there is a need for increased methodological rigor and improved study designs as small feasibility focused pilot studies far outnumber large-scale, efficacy focused randomized controlled trials (Johnson et al., 2016; Sardi, Idri, \& Fernández-Alemán, 2017). Further, as there is no single formula for successful gamification, digital game mechanics can be difficult to meaningfully integrate into health interventions. In turn, as poorly implemented mechanics generally lead to poor user experiences, cautionary tales resulting in null (e.g., DeSmet et al., 2015; Mekler, Bruhlmann, Touch \& Opwis, 2017) and iatrogenic effects (e.g., Boendermaker, Maceiras, Boffo, \& Wiers, 2016) are also well-represented within this literature. To improve the quality of future intervention gamification efforts, recommendations prescribe that selected digital game mechanics be firmly grounded in theories of human motivation (Mekler et al., 2017; Sailer, Hense, Mayr, \& Mandl, 2017; Sardi et al., 2017), aligned to the developmental and psychosocial characteristics of the target population (Koivisto \& Hamari, 
2014), endogenous to core intervention content rather than superficially or peripherally presented (Floryan et al., 2019; Johnson et al., 2016), and designed to balance hedonic and utilitarian value in the user experience (Baptista \& Oliveira, 2019).

The present effort to gamify a web-based PNF intervention for SMW is attentive to these recommendations and draws on self-determination theory (SDT; Deci \& Ryan, 1985; Deci \& Ryan, 2004; Ryan \& Deci, 2000) to inform the selection of game mechanics. SDT is a macro theory of human motivation and development that has informed the design of many commercial video games (e.g., Conway \& Elphinstone, 2017; Ulysal \& Yildirim, 2016), health behavior change interventions (e.g., Gillison, Rouse, Standage, Sebire \& Ryan, 2019; Phillips \& Guarnaccia, 2020) and gamified health behavior change interventions (e.g., Blok et al., 2019; Mekler et al., 2017; Sailer et al., 2017). Inspiring the most work in these domains is the notion that people have innate psychological needs for competence, autonomy, and relatedness (Ulysal \& Yildirim, 2016). In general, competence refers to the need for feeling that one is effective in meeting environmental demands; autonomy refers to needs for feeling authentic, acting with volition, and having input; and relatedness comprises needs for social belonging and interpersonal connection (Deci \& Ryan, 2004; Ryan \& Deci, 2000). SDT posits that basic need satisfaction fosters psychological growth, well-being, optimal functioning; and thus, people naturally tend to seek out activities that allow these needs to be satisfied (Deci \& Ryan, 2004). Findings also suggest that when these needs are met by an activity or environment, people are more likely to internalize the behaviors, attitudes, and ideas reinforced in that space (Deci \& Ryan, 2008). 


\subsection{A Gamified, Culturally Tailored, Incognito Alcohol Intervention for SMW}

Seeking to bolster intervention appeal and engagement among SMW and overcome the motivational and attentional limitations associated with traditional PNF alcohol intervention formats, drinking and stigma-coping norms were corrected within LezParlay, a social mediainspired digital competition designed to challenge negative LBQ stereotypes and increase visibility. One round of LezParlay was played each month over an eight-month period and each round featured a variable cash prize awarded to the top-scoring player exhibiting the greatest accuracy in their perceptions of LBQ peers. During the first three weeks of each round, players were invited to submit guesses about several negative stereotype-related behaviors and experiences of age and sexual identity matched LBQ peers (e.g., What percent of lesbians in their 30's own a pair of Birkenstocks? How many days per week does the typical lesbian in her $30 s^{\prime}$ drink?), select an allotment of points to wager on these guesses being true of other age and sexual identity matched players, and collect points for reporting on their own corresponding behaviors and experiences. The last week of each month, all players received individualized results for the round's topics (i.e., PNF), which included charts detailing the accuracy of the player's perceptions, how their behaviors and experiences compared to peers, and a summary of the stereotypes challenged. Detailed results concluded with a scorecard which displayed the player's point total and round score rank among all participating players and linked to a leaderboard which featured the round's top 100 scorers and revealed the round's prize winner. Notably, all actual norms featured in detailed results were derived organically from players' own reports of their behaviors and experiences.

In an effort to increase appeal and leverage culture-specific resiliencies among SMW, this novel intervention format reflected several deep structure themes including this community's 
negative LBQ stereotype related concerns (e.g., Huxley, Clarke, \& Halliwell, 2014; Fingerhut \& Abdou, 2017; Gordon, 2006), desire for increased identity visibility (e.g., Fox \& Warber, 2015; Gabbay \& Wahler, 2002; Harerra, 2018) and enjoyment of intra-community competition and sport (e.g., Davis-Delano, 2014; Dolance, 2005; Mock, Misener, \& Havitz, 2019). Further, consistent with recommendations for surface level intervention tailoring and gamified intervention development, all of the competition's game mechanics, branding, graphics, recruitment materials were jointly tailored to appeal to LBQ women in the U.S. between the ages of 21 and 59 years. The competition was also developed by an LBQ woman in the target agerange (i.e., ingroup peer) and five collaborating community organizations (i.e., HER Social App, Autostraddle, LezDoBrunch, the LA LGBT Center) trusted as sources for health and social information by SMW jointly promoted the competition online. Finally, LezParlay drew upon the nascent gamification literature (Johnson et al., 2016; Kapp, 2013; Kawachi, 2017; King, Greaves, Exeter, \& Darzi, 2013) to leverage four game mechanics associated with the satisfaction of SDT's basic psychological needs for competence, relatedness, and autonomy.

Increasing Visibility via Social Media Inspired Copresence. Within SDT, relatedness is a psychological need satisfied when a person feels connected to, and familiar with, relevant others (Deci \& Ryan, 2004; Deci, Ryan, Weiner, \& Craighead, 2010; Ryan \& Deci, 2000). In digital gaming contexts, knowledge about fellow competing players has been found to increase players' interest and enjoyment (Colwell, 2007; Lim \& Reeves, 2010; Mandryk, Inkpen, \& Calvert, 2006; Weibel, Wissmath, Habegger, Steiner, \& Groner, 2008). Accordingly, as shown in Figure 1, LezParlay players were able to create social media-like personal profiles, display avatars, and browse the profiles of fellow players in order to inform their guesses about behaviors and experiences of peers. Player profiles were intended to increase lesbian, bisexual, 
and queer female visibility_-something widely desired by SMW (Fox \& Warber, 2015; Gabbay \& Wahler, 2002). Beyond visibility, the social media like representation of peers was also expected to make the actual norms presented by LezParlay more credible and impactful. That is, a pilot study testing social media-inspired copresence features among heavy drinking college students found that alcohol PNF which framed actual norms as having been generated by the responses of peers with visible social media-like profiles produced larger reductions in drinking than did identical PNF and actual norms when peers were not visible, and norms were framed as being based on a previously collected survey data (LaBrie et al., 2019). Further, as research with lesbian-identified SMW suggests that greater social media involvement is associated with heavier drinking, inflated perceptions of peer drinking norms, and greater confidence in normative misperceptions (Boyle et al., 2020), LezParlay’s social media-inspired copresence features aimed to attract the heavy social media using SMW who stand to benefit the most from a social norms based alcohol intervention. 


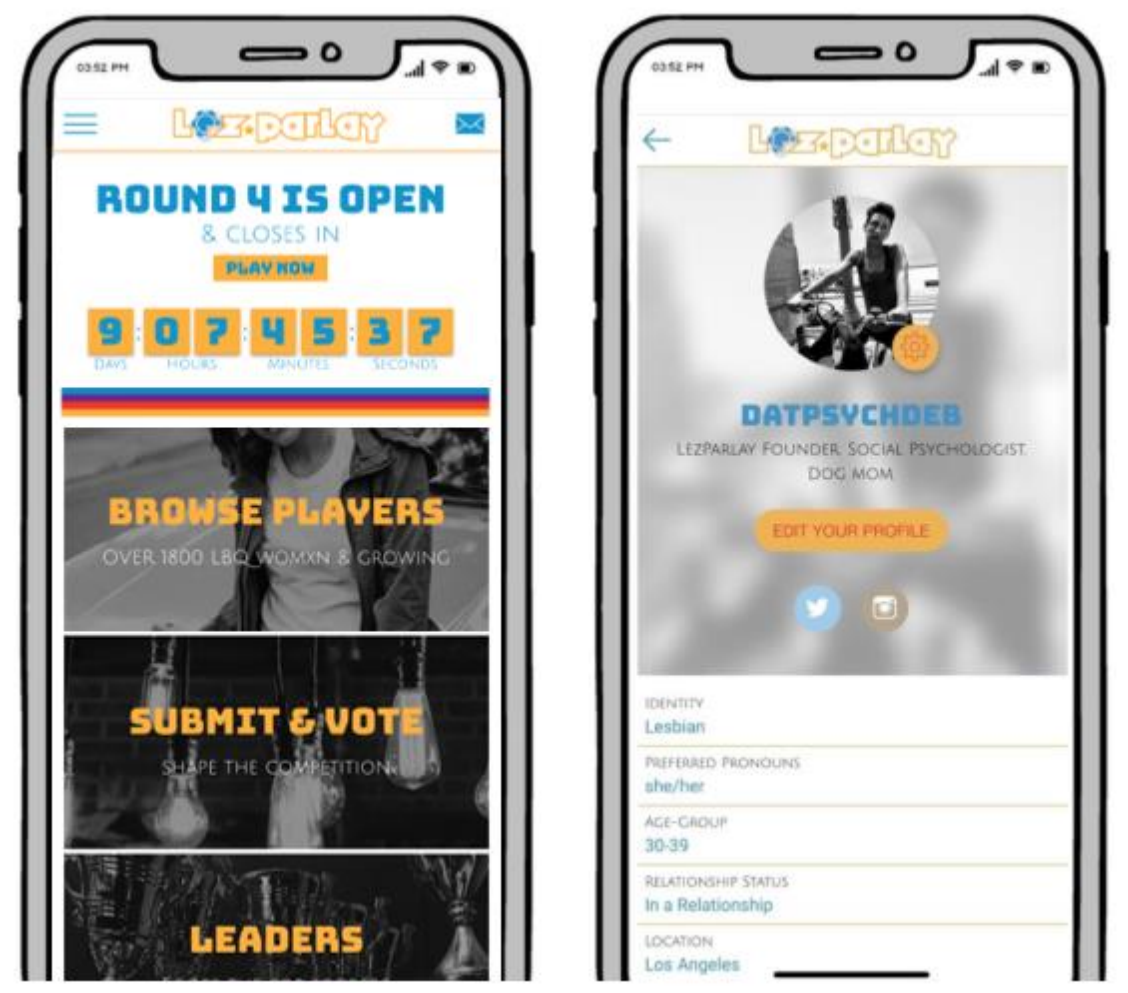

Figure 1. LezParlay app home screen and player profile

Stereotype Challenge Framing, User-generated Questions, \& Chance Elements.

Autonomy within SDT concerns a sense of volition or willingness during a task and digital games that appeal to user's social identities, interests, and values (Boyle, Connolly, \& Hainey, 2011; Przybylski, Rigby, \& Ryan, 2010; Ryan, Rigby, \& Przybylski, 2006; Uysal, Yildirim, \& Bostan, 2016) and those that offer players greater choice over the course of play tend to increase autonomy and motivation (Rigby \& Ryan, 2007; Sheldon \& Filak, 2008). LezParlay not only fostered autonomy through its packaging of a traditionally dry alcohol intervention strategy within a fun, social identity-relevant competition for SMW, but additional autonomy inducing features were leveraged in the competition to reduce heavy drinkers' psychological reactance and defensiveness when viewing PNF on alcohol use (Granfield, 2005; Steers et al., 
2016). Pilot work with college students has established that these negative reactions can be significantly reduced by providing PNF on multiple topics of interest to the target population and by giving alcohol-related PNF the appearance of being selected by chance (Boyle, Earle, McCabe, \& LaBrie, 2018). Therefore, in addition to alcohol and stigma-coping, LezParlay included questions and feedback on additional topics of interest to SMW (e.g., stereotypes related to sex, relationships, style, hobbies, pet ownership, etc.) and players were invited to help shape the competition by submitting questions to be "parlayed" in future rounds and up-voting their favorites submitted by other players. Each round of LezParlay featured several top-voted user-generated questions and began with animated spinners that determined the round's topics and amount of the round's prize. As digital game researchers theorize that the placement of chance elements immediately before intervention content may facilitate the cognitive re-framing of the content, giving it a positive, serendipitous feel (Liang, 2012; Ozcelik, Cagiltay, \& Ozcelik, 2013), LezParlay doubled down on the illusion of chance. At the end of each round, players chose between doors to unlock detailed results (i.e., PNF) on specific topics. Figure 2 provides a still overview of animated chance-based uncertainty elements in LezParlay. 

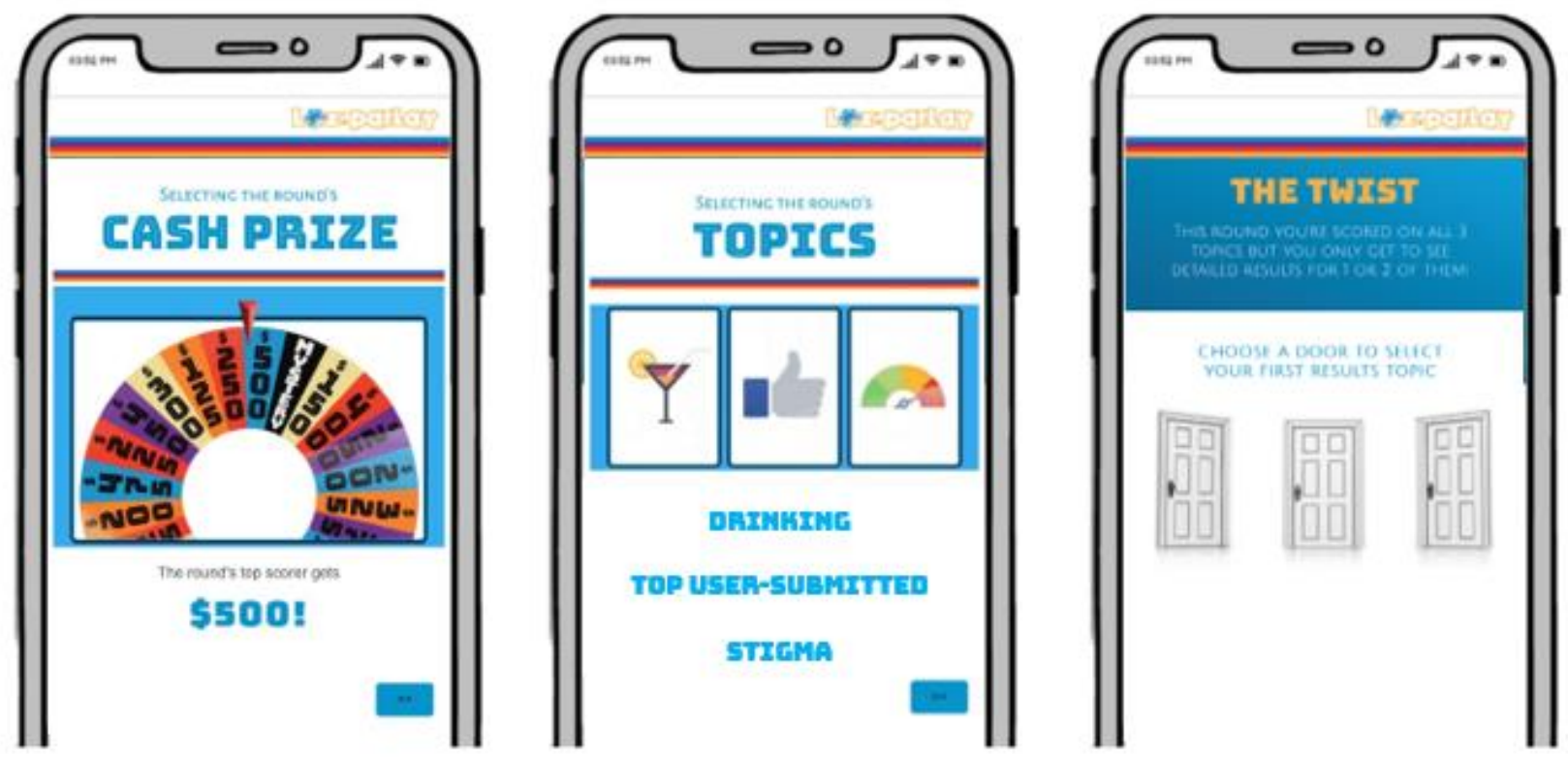

Figure 2. Chance-based uncertainty game mechanics in LezParlay

System of Points, Leaderboards \& Performance-based Prizes. In SDT, competence is a psychological need encompassing the desire for challenge as well as for feelings of capability and growth (Deci \& Ryan, 2004; Ryan \& Deci, 2000). The satisfaction of this need through frequent rewards, feedback, and the ability to track performance over time and compare performance with others is associated with greater engagement and sustained digital gameplay (Hou, 2011; van der Spek, 2012; Willson \& Leaver, 2017). Although on the surface SDT suggests that to optimize engagement, external rewards should be absent from games and interventions alike in order to foster intrinsic motivation, subsequent research has revealed nuance around the relationship between external rewards and motivation. In sum, findings suggest that external rewards can be controlling or informational in nature, and this distinction determines whether they have the effect of undermining, maintaining, or enhancing intrinsic motivation (Ryan \& Deci, 2008; Uysal \& Yildirim, 2016). For instance, in digital games, performance-based external rewards (e.g., points, leaderboards, top scorer prizes, etc.) are often 
utilized to foster competition and provide key information about task competence and how one's performance compares to that of relevant others', thus supporting competence needs (Conway \& Elphinstone, 2017; Uysal \& Yildirim, 2016). The degree to which these types of external rewards are sought through an activity/task is also autonomous. That is, a video game can be played for fun or practice purposes to the extent that one is not concerned with "winning", or players can engage competitively in order to pursue social recognition and prizes. Because these types of external rewards support competence and autonomy needs, they have been found to maintain or enhance intrinsic motivation (Ryan \& Deci, 2008; Uysal \& Yildirim, 2016).

To satisfy competence needs, LezParlay invited SMW to guess about the behaviors and experiences of the typical player sharing their sexual identity and age-group, wager points on the degree to which they think their guess is correct based on the answers submitted by these players, and earn bonus points for reporting on their own parallel behaviors and experiences. Points were won and lost in LezParlay based on the accuracy of guesses and sizes of wagers. Leaderboards within the app displayed the Top 100 scorers following each round of play and variable cash prizes were awarded to top scorers. Further, select rounds featured a "Replay Bonus" which allowed players to replay a topic they previously received PNF on, thereby boosting their score by demonstrating increased competence. 

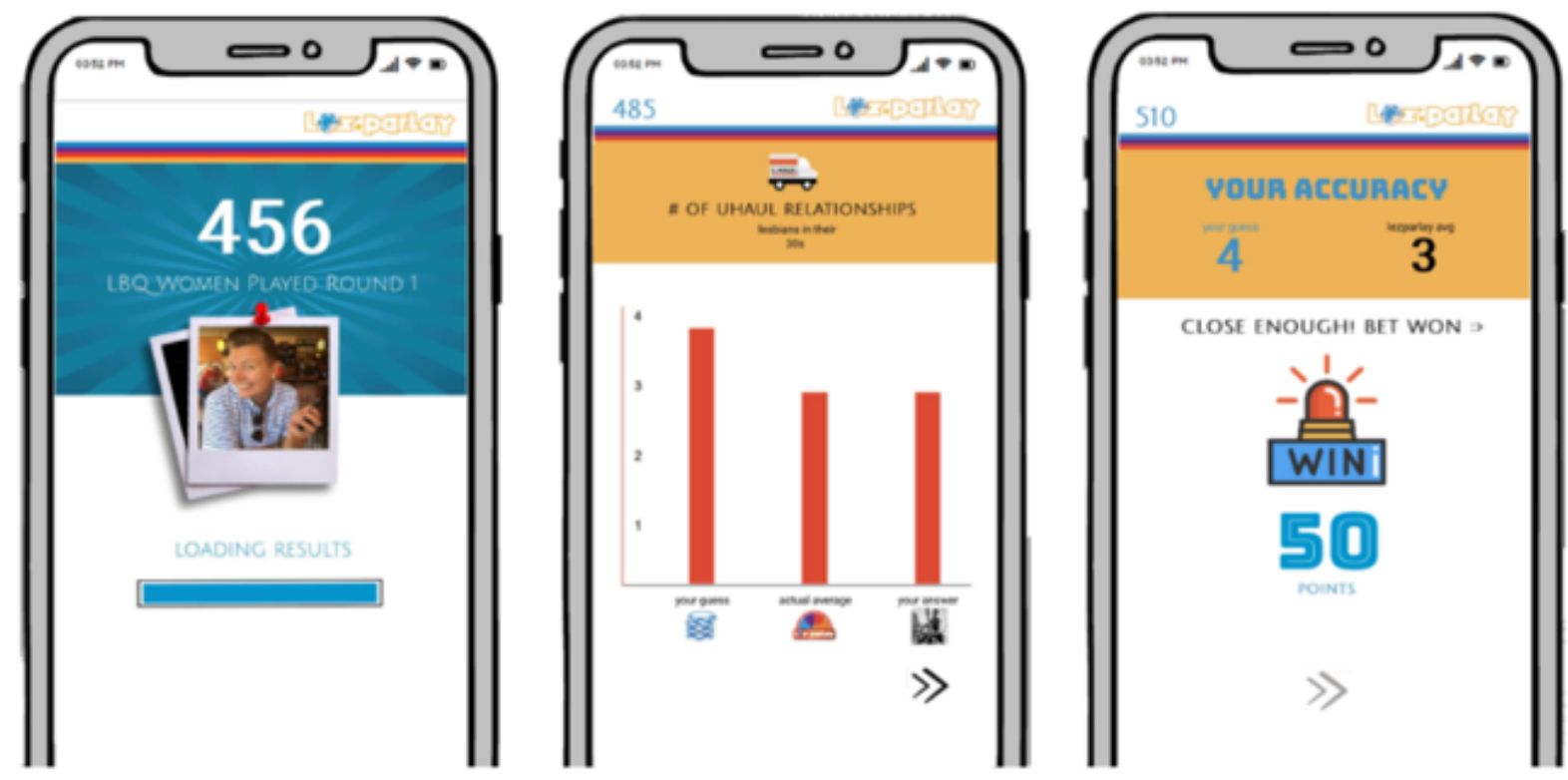

Figure 3. Detailed results and wager-based scoring in LezParlay.

\subsection{The Current Study}

Following recent recommendations for testing the real-world feasibility and impact of normative feedback interventions (Dempsey et al., 2018; Radtke, Ostergaard, Cooke, \& Scholz, 2017), the LezParlay intervention was examined through a Type I Hybrid-EffectivenessImplementation Trial (Curran, Bauer, Mittman, Pyne, \& Stetler, 2012; Landes, McBain, \& Curran, 2019). That is, in contrast to recruiting SMW into a transparent, incentivized, alcohol intervention study, LezParlay was advertised to SMW as it would be in the real-world - as a free, online competition designed to test lesbian, bisexual, and queer stereotypes and increase visibility. Then, after several rounds of play, a sub-sample of 500 drinkers already taking part in the competition were invited to take part in an evaluation study wherein they were incentivized to play subsequent rounds and complete a short feedback survey following the competition. Importantly, study participants were discreetly randomized to receive 1 of 3 unique sequences of feedback (i.e., Alcohol \& Stigma-Coping, Alcohol \& Control, or Control topics only) over two consecutive rounds of play. Reductions in drinking were assessed two months later, not through 
a transparent alcohol-focused follow-up survey plagued by demand characteristics, but rather, within the competition through a "Replay Bonus" round which invited all players to boost their scores by guessing, betting, and reporting on alcohol use and control topics a second time. Then, following the competition, evaluation study participants completed a feedback survey assessing competition acceptability, perceived benefits, and ideas/requests for the next version of LezParlay.

\section{Evaluating LezParlay Intervention Efficacy}

The efficacy of PNF on alcohol use and stigma-coping behaviors delivered by LezParlay was examined and analysis of moderators sought to identify the characteristics of SMW for whom treatment PNF was most and least efficacious. Five specific hypotheses were evaluated:

(H1) Relative to LezParlay players randomized to receive control PNF, those randomized to receive alcohol only PNF (a) and alcohol+coping PNF (b) will report reduced alcohol use at follow-up.

(H2) LezParlay players randomized to receive PNF on alcohol+coping will reduce their alcohol use to a greater degree than will those randomized to receive PNF on only alcohol use.

(H3) Intervention efficacy will be moderated by recent interpersonal stigma exposure such that (a) drinking reduction effects associated with randomization to a treatment PNF condition will be enhanced when interpersonal stigma exposure is high following PNF delivery (relative to low); further (b) the alcohol+coping condition will outperform the alcohol only condition in reducing alcohol use when interpersonal stigma following PNF delivery is high (relative to low). 
(H4) Intervention efficacy will be moderated by structural stigma exposure such that (a) drinking reduction effects associated with randomization to a treatment PNF condition will be enhanced when structural stigma exposure is high (relative to low); further, (b) the alcohol+coping condition will outperform the alcohol only condition in reducing alcohol use when structural stigma is high (relative to low).

(H5) Intervention efficacy will be moderated by baseline alcohol use such that (a) the drinking reduction effects associated with treatment PNF are enhanced among participants who enter the competition as heavier (relative to lighter) drinkers; further, (b) the alcohol+coping condition will outperform the alcohol only condition in reducing alcohol use among participants who enter the competition as heavier (relative to lighter) drinkers.

In addition to these specific hypotheses, four additional demographic variables were examined as potential moderators of PNF efficacy: participant age, sexual identity, relationship status, and race/ethnicity. As there was no literature to support specific hypotheses due to the uniqueness of this population in the PNF intervention context, the examination of these potential moderators was exploratory.

\section{Evaluating LezParlay Intervention Feasibility}

Six critical questions related to LezParlay's feasibility as an alcohol intervention strategy were also examined using a combination of data collected from the backend of the LezParlay application, Google Analytics, and feedback surveys completed by drinkers taking part in the evaluation study:

Reach and Origin: How many SMW signed up to take part in the LezParlay competition, what were their demographic characteristics, and which channels for promotion/recruitment brought them to the app? 
Engagement: How many times did players login to the LezParlay web app, how many rounds of the competition did players complete, and what section of the app did players engage with the most?

Player Alcohol Use: How effective was LezParlay in attracting moderate and heavy drinking SMW most likely to benefit from a PNF intervention as well as non- and light drinking SMW so that risk-reducing drinking and coping norms could be generated in real-time from round data?

Acceptability \& Perceived Benefits: Did drinkers recruited into the evaluation study find the LezParlay competition acceptable and psychologically beneficial?

Requested Changes and Additional Features: What changes to Lezparlay's competition format and web app were desired by drinkers taking part in the evaluation study? 


\section{CHAPTER 3}

\section{Method}

LezParlay was a low-cost, device responsive, HTML5 progressive web application (PWA) integrated with Facebook Connect, Qualtrics Research Suite, Construct 3 Game Engine, as well as a text messaging API and email server. Although providing a native app-like feel on Android and Apple smartphones, players were not required to visit an app store to download any software. Instead, SMW simply accessed the LezParlay web app at https://www.lezparlay.com and a pop-up message provided simple, one-step instructions on saving the web app in order to make LezParlay easily accessible from the home screen of their device (e.g., smartphone, tablet, computer).

\subsection{Competition Recruitment}

The LezParlay competition was open to all lesbian, bisexual, and queer-identified women who were 21 years or older and currently residing in the United States, regardless of birth sex. Players learned about the competition through 1 of 4 recruitment strategies taking place over a 3month period. First, prior to the launch of the first round, local SMW were invited to sign-up through flyers distributed at several lesbian community events in Los Angeles. Next, as the first round began, marketing campaigns on HER Social App, the leading dating/social app for queer women, invited users in their three largest markets to LezParlay via push notifications and in-app advertisements. At this time, an advertisement was also placed in the newsletter of Autostraddle, the leading independently owned news website for queer women, and targeted campaigns on Facebook, Instagram, and Google began promoting the LezParlay competition to LBQ women residing all over the United States. All recruitment materials described LezParlay as a free competition designed to test LBQ stereotypes and increase visibility. Importantly, although most 
advertisements and flyers noted the performance-based cash prizes to be awarded to top scorers, there was no mention of the evaluation study to take place in later rounds or the participationbased incentives to be offered to players electing to take part in the study. Thus, at the point of sign-up, all players were choosing to take part in a competition for a few cash prizes rather than participate in a paid research study. See Appendix A for recruitment materials (i.e., promotional video, social media ads, app notification invite, flyers distributed locally). All materials linked to LezParlay's informational landing page (http://lezparlayla.com/), which explained how the competition works, presented answers to frequently asked questions, and provided a sign-up button that redirected interested women to view and accept the Terms of Service and Privacy Policy (basic informed consent for competition participation, See Appendix B) before creating their accounts. All recruitment procedures and materials were approved by the Institutional Review Board at Loyola Marymount University.

\subsection{Procedure}

After accepting the Terms of Service and Privacy Policy, new users created their accounts by providing a mobile phone number and signing up with either a valid email address/password combination or using their existing Facebook credentials. Next, players completed their LezParlay public profile, which included their sexual identity (lesbian, bisexual, queer), age-group (i.e., 20s, 30s, 40s, 50s or older), and relationship status (single, it's

complicated, in a relationship, married). Although these initial profile fields had to be completed in order to take part in the competition, users also had the options of uploading a profile photo or bitmoji to represent them, entering a brief textual self-description, selecting their pronouns (she/her, he/him, they/them) and connecting their Facebook, Twitter, and/or Instagram accounts to connect with other players. Following profile creation, players were directed to a home screen 
which displayed an animated countdown to the end of the current round, as well as large buttons to play the current round, browse the profiles of other players, submit and vote on questions to be parlayed in future rounds, view round winners and leaderboards, edit their public profile, and change account settings (see Figure 1).

Competition Round Play. Each round began with a parlay period, which spanned the first three weeks of the month. Players logged in during this period to submit their guesses about other players, set their wagers, and report on their own parallel behaviors and experiences. Each round was designed to take no longer than 6 minutes to complete and began with an animated slot machine-like spinner selecting 2-3 topics on which all players would answer questions. Following topic selection, an animated wheel of fortune type spinner then selected the amount of the round's top scorer cash prize. For each topic, 3-4 questions prompted players to guess about the behaviors and experiences of other players sharing their sexual identity and age-group (e.g., "How many flannel shirts does the typical lesbian in her 20s own? "How many days per week does the typical lesbian in her 40s drink?") and select a number of points to wager (i.e., 10, 25, $50,100)$ on this guess being correct based on the actual, average response of players sharing her sexual identity and age-group. Next, players reported on their own behaviors and experiences by answering parallel items (e.g., "How many flannel shirts do you own?”, How many days per week do you drink?). At the end of the parlay period, the round closed and actual norms were derived from players' reports of their own behaviors and experiences. The average response from all players in each age and sexual identity group to each question about players' own behavior/experiences became the correct answers (i.e., the actual norms in PNF) featured in detailed results at the end of the month, with point wagers won and lost accordingly. See Table 1 for a summary of the question topics and cash prizes across competition rounds. 
Table 1.

Summary of question topics and PNF delivery across rounds of the LezParlay competition.

\begin{tabular}{ccc}
\hline Round \# & Top Scorer Prize & Question Topics \\
\hline 1 & $\$ 200$ & Pets \& Uhauling \\
2 & $\$ 300$ & Style \& Identity Experiences \\
$\mathbf{3}$ & $\mathbf{\$ 5 0 0}$ & User-Submitted, Drinking, \& Stigma \\
$\mathbf{4}$ & $\mathbf{\$ 4 0 0}$ & User-Submitted, Coping, \& Relationships \\
5 & $\$ 200$ & User-Submitted \& Relationship Infidelity \\
6 & $\$ 300$ & User-Submitted, Politics, \& Coming Out \\
$\mathbf{7}$ & $\mathbf{\$ 5 0 0}$ & Drinking \& Stigma (REPLAY BONUSES) \& Sex \\
8 & $\$ 400$ & Uhauling \& Pets (REPLAY BONUSES), Health \\
\hline
\end{tabular}

Note. Bolded rounds 3, 4, and 7 were critical to the efficacy study. Condition assignment determined whether PNF on treatment or control topics were delivered at the end of Rounds 3 and 4.

Detailed Results (PNF). Construct 3 game engine was programmed to create private URLs at which players could view animated, personalized, detailed results. Players were text messaged their private results link at the end of each month. Upon opening this link on a smartphone, tablet, or computer, an initial screen detailed the player's total score and wagers for the round. Next, the total number of players who completed the round and thereby contributed to correct answers (i.e., actual norms) were displayed alongside an animated reel of avatars representing these players. Then, results for each question were presented through a series of screens, with the first reiterating the question. Then, consistent with PNF, the second screen displayed an animated, horizontal bar chart with 3 bars contrasting: 1) the player's guess about the answer of other players' in her age and sexual identity group (perceived norm); 2) the correct answer based on the actual responses of players in her age and sexual identity group (actual norm); and, 3) the players own answer (own behavior). Following this bar chart, animations and sound effects revealed the player's win or loss of points based on the accuracy of her guess and point wager. After these screens were displayed for each question within a topic, a final screen 
presented a chart revealing how the player's behavior for the topic compared to other players across age groups and sexual identities. This was accomplished by super-imposing the player's avatar over a chart summarizing the distribution of responses. Following all topic-specific screens, a summary screen highlighted relevant stats that shattered or confirmed stereotypes related to the round's topics and a stats screen provided an overview of the player's performance. Buttons at the bottom of this stats screen allowed players to view the round's top 100 scorers in a leaderboard and begin the next round.

Evaluation Study Enrollment. There was no upper limit on the number of SMW who could take part in the competition and the goal was to recruit 1,200 lesbian, bisexual, and queer women to take part during the first three monthly rounds so that the pool of players would include a sufficient number of drinkers and allow for stable actual norms for each sexual identity and age-group to be computed. Then, from this larger pool of players, 500 drinkers were to be recruited into the LezParlay evaluation study (RCT) upon completing Round 3. Acting as baseline (T1), this round featured questions about alcohol use, sexual minority stigma experiences, and two sets of non-health, stereotype related control questions submitted by players. From the player perspective, this round looked no different than previous rounds in terms of format, and no announcement of the evaluation study was made prior to round play. However, upon submitting answers to alcohol questions, all players were covertly screened for evaluation study eligibility based on their answers to alcohol use items (i.e., number of drinking days per week and peak drinks on a single day during the past two months) as well as their number of previous rounds played (automatically pushed into Qualtrics from the web app's backend). Those who played at least one previous round, and reported drinking alcohol on three or more days per week OR having three or more drinks on their peak drinking occasion, were 
invited to take part in the evaluation study at the end of the round via a pop-up invite message. Interested players advanced to an informed consent page, which explained that the goal of the study was to evaluate the impact and format of detailed results received in LezParlay and gather player feedback to inform the next version of the competition. Information also explained that participation in the evaluation study involved playing and viewing detailed results in Rounds 3, 4, and 7, then completing a brief feedback survey at the end of the competition. Further, participants could earn up to $\$ 60$ in e-gift cards of their choice ( $\$ 20$ following Round 4, $\$ 20$ following Round 7, and $\$ 20$ following the feedback survey) for taking part in the study. Players who checked boxes indicating that they understood what study participation entailed and desired to participate were welcomed into the study as "official testers" of LezParlay. A total of 1337 SMW completed Round 3, and 912 of these players were screened for eligibility before the 500 spots in the evaluation study were filled. Figure 4 details participant numbers through screening, enrollment, the 2-month post-intervention follow-up, and post-competition feedback survey. One participant who screened into the study and was subsequently randomly assigned to the alcohol+coping condition contacted the investigator to disclose that she was a minor and had lied about her age in order to take part in LezParlay and the evaluation study (See Appendix C). This participant was removed from the study (and competition) prior to the delivery of intervention feedback, resulting in a baseline sample of 499 SMW. 


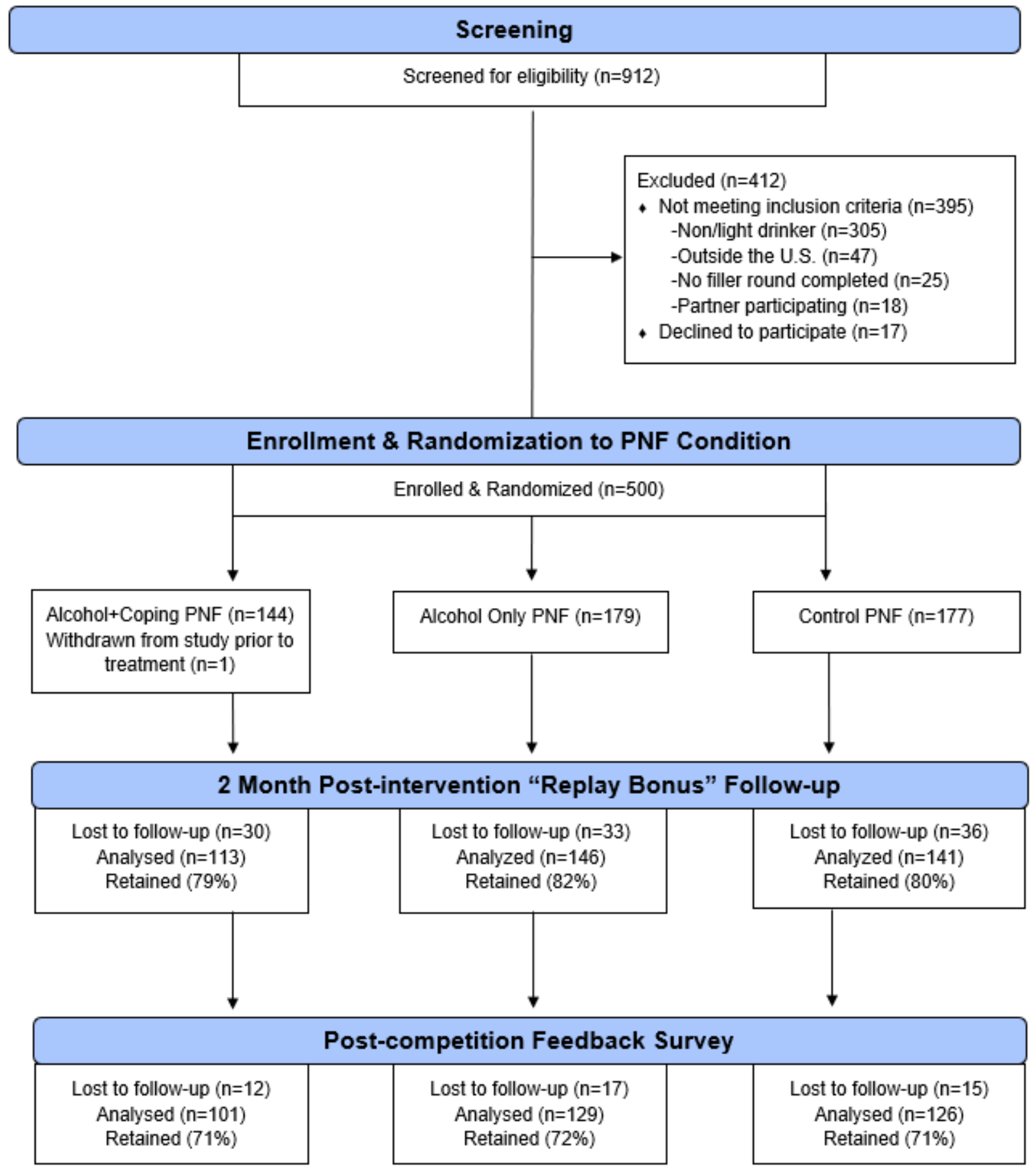

Figure 4. Flow of participants through screening, enrollment, the 2-month post-intervention follow-up, and the post-competition feedback survey. 
RCT Design, Randomization, \& Debriefing. Upon joining the study, participants were randomized to a study condition through Qualtrics Research Suite's randomizer to receive 1 of 3 specific sequences of feedback topics across Rounds 3 and 4: alcohol+coping, alcohol+control, or control only. When prompted to choose between doors to determine their detailed results topics in Rounds 3 and 4, evaluation study participants' doors were "fixed" to open to their assigned feedback topic regardless of the door selected. Upon completing the feedback survey at the end of the competition, study participants were debriefed regarding the study's research questions and the fixed sequences of health or non-health-related feedback they were randomized to receive in Rounds 3 and 4 of the competition (See Appendix F for the debriefing statement).

Intervention Rounds 3 and 4. All players taking part in the 3rd round of LezParlay estimated the drinking behaviors of the typical, same sexual identity player in their age-group during the previous two months, reporting on their perceptions of this typical player's (1) maximum number of drinks consumed on a single occasion, (2) average number of drinks consumed per occasion, and (3) average number of drinking days per week (i.e., the Quantity, Frequency, Max measure; Baer, 1993). Players also estimated the number of negative-alcoholrelated consequences experienced over the previous two months by the typical player in their sexual identity and age-group from a list of 8 negative consequences (i.e., had a hangover or illness, got in a physical or verbal fight, had problems with significant other, missed a social engagement or event, had problems with friends or family, performed poorly at work or school, had an unwanted or regrettable sexual experience). Then, players answered parallel items assessing their own drinking and consequences over the corresponding two-month period. All players taking part in Round 4 of the competition were prompted to think about how other players deal with stress and sexual minority stigma and were asked to estimate the percent of the 
time (i.e., 0-100\%) the typical player in their sexual identity and age-group tried to feel better during the past month by: (1) drinking alcohol; (2) taking a drug; (3) meditating, using relaxation techniques, or exercising; and, (4) talking to a close other or mental health professional. Then, players were prompted to think about how they, themselves, deal with stress and stigma and respond to parallel items. The actual norms variably delivered to evaluation study participants in PNF at the end of Rounds 3 and 4 were derived by computing the actual average response among all players submitting responses in each sexual identity and age-group. Although this method of deriving actual norms allowed for anticipated variability by sexual identity, the actual norms within each age-group were consistent across the three sexual identity groups (see Table 2).

Table 2.

Competition derived actual norms for intervention topics by age-group

\begin{tabular}{lccc}
\hline & & Age-Group & \\
& $21-29$ years & $30-39$ years & 40 years+ \\
\hline Round 3- Alcohol Use Actual Norms & $n=627$ & $n=498$ & $n=212$ \\
\hline Drinking days per week & 2 & 2 & 1.5 \\
Average drinks per occasion & 2.5 & 2 & 2 \\
Peak drinks on one occasion & 4 & 3 & 3 \\
Number of Negative Consequences & 2 & 1.5 & 1 \\
& & & \\
\hline Round 4- Coping Actual Norms & $n=503$ & $n=414$ & $n=186$ \\
\hline$\%$ of time drink alcohol to cope & 18 & 17 & 16 \\
\% of time use drug to cope & 12 & 9 & 9 \\
$\%$ of time exercise, meditate, to cope & 55 & 61 & 49 \\
$\%$ of time seek social support to cope & 53 & 50 & 62 \\
\hline
\end{tabular}

Participants randomized to receive PNF on control topics received similarly-formatted PNF screens detailing results for non-health-related topics in Round 3 (i.e., household repair ability, home improvement store visits, toolbox ownership, scissoring) and Round 4 (i.e., time in between relationships, texting exes, couples being confused for sisters, watching The L Word television series). Example detailed results on one non-health-related control topic (user- 
submitted) and one treatment topic (alcohol use) can be viewed from any Wi-Fi-connected Apple or Android smartphone at https://lezparlayresults/demo.

\subsection{Measures}

\section{Efficacy Measures}

Perceived Norms for Alcohol Use and Negative Consequences. As described in the previous section, perceived drinking norms and were assessed organically in the competition in Rounds 3 (baseline) and 7 (follow-up) by items modeled after Baer's (1993) Quantity, Frequency, Max measure. Response options for the norm item assessing the typical player's number of drinking days per week ranged from 0 to 7 days, whereas response options for items assessing the typical players' average drinks per occasion and max drinks consumed on one occasion ranged from 0 to 12 drinks. Perceived negative alcohol-related consequences were assessed by an additional item which presented a list of 8 negative consequences and prompted participants to guess the number of these consequences (0-8) experienced by the typical player. At both time-points, all norm items referenced the previous two month period and the sexual identity and age-group of the typical player in question were piped to match each participant's sexual identity and age-group (e.g., Over the past two months, on average, on how many days per week did the typical [lesbian] in her [30's] drink? During the past two months, how many of the negative consequences above did the typical [lesbian] in her [30s] experience due to drinking/partying?). The four norm items were positively correlated and exhibited a high degree of internal consistency $(\alpha>$.75). As such, composite measures of perceived drinking norms at baseline and follow-up were computed by z-scoring, then averaging across items at each time point. 
Own Alcohol Use and Negative Consequences. Participants own alcohol use and negative consequences over the past two months were assessed in Round 3 (baseline) and 7 (follow-up) by items that paralleled norm items (e.g., Over the past two months, on average, on how many days per week did YOU drink?) and presented the same response options (i.e., 0-7 days; 0-12 drinks; 0-8 negative consequences). The four items exhibited high internal consistency at baseline and follow-up $(\alpha>$.80). As such, composite measures of alcohol use behavior were computed by z-scoring then averaging across items at each time point. In addition to these composites, three key behaviors of interest in alcohol intervention research were examined individually pre- and post-intervention: 1) estimated number of drinks per week over the previous two months (computed by multiplying reported number of drinking days per week and average number of drinks per occasion at each timepoint); 2) peak number of drinks consumed on one occasion over the previous two months; and, 3) number of negative alcoholrelated consequences experienced over the previous two months.

Interpersonal Stigma Exposure. Interpersonal stigma exposure was also assessed at baseline (Round 3) and follow-up (as a Replay Bonus topic in Round 7). Players guessed about the stigma experiences of other players and reported on their own stigma experiences over the previous two months. Stigma norms were not corrected in the competition; these perceptions were only assessed so that it made sense for players to report on their own recent interpersonal stigma exposure (a theorized moderator of conditional effects on drinking) at the same point in time that they were reporting on their alcohol use and negative consequences in the game. Players' own interpersonal stigma exposure was assessed by their responses to two items varying in severity: (1) During the past two months, how many times have you been physically harmed due to your sexual identity? (2) During the past two months, how many times have you been 
harassed or threatened (online or in-person) due to your sexual identity? In examining the raw response frequencies for these items at $\mathrm{T} 1$ and $\mathrm{T} 2,>75 \%$ were concentrated in the range of 0 to 1. Therefore, items were recoded to reflect this binary (i.e., $0=$ this did not happen; $1=$ this happened one or more times) at each timepoint. As responses to pairs of items at each time point were significantly associated, interpersonal stigma scores at $\mathrm{T} 1$ and $\mathrm{T} 2$ were derived by summing responses to the two items at each time point so that scores ranged from 0 to 2 . Timepoint specific measures are separately tested as moderators of intervention efficacy. However, because timepoint specific measures were also correlated $(r=.31)$, a total measure was created (i.e., timepoint specific scores were summed) for covariate use in tests of main effects in order to reduce multi-collinearity.

Structural Stigma Exposure. The geolocations (approximate latitudes and longitudes) of study participants at the point of study enrollment were recorded by the LezParlay web app. These location coordinates were then exported to a CSV file along with participant identification number (all identifying information removed) and imported into the Kloudend Ipapi API in order to fetch the state and county of each study participant. Then, consistent with previous structural stigma research (e.g., Hatzenbuehler, 2014; Hatzenbuehler \& Link, 2014; Hatzenbuehler et al., 2015) and summarized in Table 2, publicly available secondary data indicative of public opinion toward sexual minorities, equality-related legislature and policies, population densities of samesex couples, and presence of LGBTQ community resources were merged into the dataset based on participants' states and counties. As used in previous structural stigma research (Hatzenbuehler, 2014; Hatzenbuehler \& Link, 2014; Hatzenbuehler et al., 2015), public opinion toward sexual minorities at the state-level was merged in from data published by Lax and Phillips (2009). These researchers derived public opinion scores for each state by aggregating 41 
random national polls conducted by various organizations between 1999 and 2008 that assessed attitudes toward gay adoption, hate crimes, health benefits, discrimination in jobs and housing, marriage, sodomy, and civil unions. To assess the presence of discriminatory/unsupportive legislature at the state-level, legislative data from the Movement Advancement Project (2019) were utilized. For each state, an overall policy tally counted the number of laws and policies related to relationship and parental recognition, nondiscrimination, religious exemptions, LGBT youth, health care, criminal justice, and identity documents within the state that help drive equality for sexual and gender minority people. The number of same-sex couples per 1,000 households in each state was also merged in from Movement Advancement Project data. At the county-level, population densities of same-sex couples was merged in from U.S. Census data adjusted by the William's Institute (LGBT Demographic Data Interactive, 2019). To measure political conservatism at the county-level, republican/democrat voting differentials in the 2016 U.S. Presidential election were merged in from collated TownHall.com data (McGovern, 2018). Finally, to assess the presence of LGBTQ-specific community resources at the county-level, the number of LGBTQ community centers in each county were coded and merged in from the Centerlink LGBTQ Community Center Directory (2019). County-specific google searches were also conducted to double-check for the presence of centers in participant counties. Appendix D provides an overview of data cleaning procedures for individual indices and factor analytic results examining loadings for state and county-level indices. Based on factor loadings, separate state ( 3 items; $\alpha=.87$ ) and county ( 3 items; $\alpha=.81$ ) specific measures of structural stigma were computed by summing across each set of indices. These state and county-level measures were computed for use in tests of moderation (each measure tested separately without controlling for the other measure). However, due to the strong correlation between state and county-level scores 
$(r=.58)$, a total structural stigma score was also computed by averaging across the six state and county indices $(\alpha=.86)$. This total measure was created for covariate use in tests of main effects in order to reduce multi-collinearity.

\section{Feasibility Measures}

Reach and Engagement. Data from Google Analytics and the application's backend were used to examine the demographic characteristics, states of residence, average number of logins, page views, and total number of rounds played among all SMW who signed up to take part in the LezParlay competition.

Player Alcohol Use. Traditional PNF interventions typically deliver actual drinking norms from previously collected large survey samples which include drinking and non-drinking members of the target population. As LezParlay diverges from this approach in transparently deriving timely actual norms from the responses of players taking part in each round of the competition, it is important to examine the degree to which LezParlay is able to similarly engage non and light drinking SMW, in order to bring down the drinking norms, as well as the moderate and heavy drinking SMW most likely to benefit from having their peer drinking norms corrected. To this end, examination of drinking data collected in Round 3 ( $n=1337$ study participants and non-participant players) allowed for the proportions of players who began the competition at different levels of alcohol use (non/light drinkers, moderate drinkers consuming no more than 7 drinks per week and no more than 3 drinks per day, and high-risk heavy drinkers exceeding guidelines from the National Institute of Alcohol Abuse and Alcoholism) to be examined.

Acceptability. The feedback survey prompted evaluation study participants to rate key aspects of the competition (e.g., the stereotype challenge concept, topics and questions, detailed results, leaderboards, the ability to browse player profiles, the ability to submit questions, the 
ability to bet points on the accuracy of guesses, etc.) on Likert type scales ranging from disliked very much (1) to liked very much (5). A total acceptability score was derived by computing the average rating among items assessing individual aspects of the competition.

Perceived Benefits. A single yes/no item in the feedback survey asked participants whether they felt participating in the LezParlay competition was psychologically beneficial. Those indicating yes to this question were presented the option of entering text to describe perceived benefits.

Improvements \& Requested Features. A final free response item asked participants to share any recommendations they have for improving the competition and detail any features they would like to see in the next version. 


\section{CHAPTER 4}

\section{Efficacy Results}

\section{Overall Analytic Approach}

At the 2-month follow-up, roughly $20 \%$ of participants in each condition were lost to attrition. These players were not able to be reached via email or text message to play follow-up Round 7 and the majority exhibited no web app logins between Round 5 and Round 8, suggesting that they stopped taking part in the larger competition. Further, although IP addresses were matched to confirm that all study participants viewed Round 3 PNF on assigned alcohol/control topics, there were a portion of participants lost to follow-up (8\%) for whom views of Round 4 coping/control PNF could not be verified. To address these issues, gold standard recommendations for attrition and treatment departures in alcohol intervention studies (Hallgren \& Witkiewitz, 2013; Witkiewitz et al., 2014) were followed. Intent to treat (ITT) is an analytic strategy for reducing potential bias in treatment effects arising from missing data in randomized controlled trials (RCTs). Central to this approach is the idea that all individuals randomized to a study condition should be included in the analysis, in the groups to which they were randomized, regardless of departures from randomized treatment and study attrition (White, Horton, Carpenter, \& Pocock, 2011; White, Carpenter, \& Horton, 2012). Adopting this approach in the current study, traditional complete case (CC) models first estimated treatment effects among followed participants who were randomized to a study condition and completed the 2month follow-up ( $n=400)$. Then, missing outcome data among participants assigned to a study condition but lost to follow-up were derived via multiple imputation. That is, each missing outcome value was imputed 5 times based on observed baseline data (i.e., demographic characteristics, interpersonal and structural stigma exposure, drinking norms, and alcohol use) 
using the multiple imputation add-on for SPSS v26. This package uses an MCMC algorithm known as fully conditional specification (FCS) or chained equations imputation. For each missing value, the pooled estimate of the 5 imputed values were utilized in parallel ITT models that more conservatively examined effects using data from all participants randomized to a study condition $(n=499)$. For tests of hypotheses, the in-text presentation of results is focused on CC models. However, to minimize bias and better inform conclusions about intervention efficacy, results from ITT models are presented in Appendix E, and effect sizes derived from ITT and CC analyses are compared in-text. For published examples of this approach in the context of RCTs evaluating PNF alcohol interventions see Bedendo et al. (2019b), Pedersen et al. (2017), and Schulz et al. (2013).

\subsection{Preliminary Analyses}

\section{Missing Data}

Attrition was not significantly associated with study condition, sexual identity, perceptions of drinking norms at baseline, alcohol use at baseline, or stigma-related variables. The only variables significantly associated with attrition were participant age in years and race/ethnicity. Participants lost to follow-up were likely to be younger, $t(497)=4.48, p<.001$, and non-Hispanic white, $t(497)=4.13 p<.001$, reflecting majority sample characteristics (i.e., the “average" participant was both younger and non-Hispanic white) and suggesting that attrition was random rather than systematic. Beyond attrition, there were no other missing data among participants completing baseline and follow-up rounds.

\section{Descriptive Statistics Overall and by Study Condition}

Participant demographics for the sample overall and by study condition are presented in Table 4 and descriptive statistics for perceived drinking norms and alcohol use behaviors at 
baseline $(n=499)$ and follow-up $(n=400)$ are presented in Table 5. Overall, evaluation study participants ranged in age from 21 to 55 years, with the average participant roughly 30 years old $(M=29.87 S D=7.32)$. Participants were geographically diverse, taking part in the LezParlay competition from 44 U.S states and 221 different counties. As evident from Table 4, the sample was also diverse in terms of ethnicity (25\% Hispanic), race (46\% racial minority) and sexual identity (58\% identified as lesbian, $23 \%$ bisexual, and $19 \%$ queer). To determine whether randomization was successful, demographic characteristics, stigma variables, perceived alcohol use norms, and alcohol use behaviors assessed at baseline were assessed for conditional equivalency via one-way ANOVAs (continuous measures) and chi-square tests (categorical measures). Indicative of successful randomization, no significant between-condition differences in any of these variables were detected at baseline among the full $(n=499)$ nor followed samples $(n=400)$.

In contrast to the lack of between-condition differences at baseline, one-way ANOVAs revealed a clear pattern of between-condition differences in composite measures of postintervention perceived drinking norms, $F(2,397)=131.13, p<.001$, and alcohol use $F(2,397)$ $=21.15, p<.001$. Parallel conditional differences were also observed across individual norm and alcohol use measures at follow-up. As flagged in the bottom of Table 5, relative to control, both treatment PNF conditions were associated with decreased perceived drinking norms and alcohol use at follow-up, suggesting preliminary support for H1. In contrast, the lack of significant differences between the two significant treatment conditions on these outcomes suggest an initial lack of support for $\mathrm{H} 2$. 
Table 4

Demographic and psychosocial characteristics of study participants overall and by condition assignment

\begin{tabular}{|c|c|c|c|c|c|c|c|c|}
\hline & \multicolumn{2}{|c|}{$\begin{array}{c}\text { Overall } \\
(N=499)\end{array}$} & \multicolumn{2}{|c|}{$\begin{array}{l}\text { Control PNF } \\
(n=177)\end{array}$} & \multicolumn{2}{|c|}{$\begin{array}{l}\text { Alcohol PNF } \\
\quad(n=179)\end{array}$} & \multicolumn{2}{|c|}{$\begin{array}{c}\text { Alcohol + Coping PNF } \\
(n=143)\end{array}$} \\
\hline \multicolumn{9}{|l|}{ Sexual Identity } \\
\hline Bisexual & 23 (115) & & $27(48)$ & & $22(39)$ & & $20(22)$ & \\
\hline Queer & $19(94)$ & & $20(35)$ & & $18(32)$ & & $18(22)$ & \\
\hline \multicolumn{9}{|l|}{ Relationship Status } \\
\hline Single & $42(209)$ & & $45(80)$ & & $39(69)$ & & $42(60)$ & \\
\hline \multicolumn{9}{|l|}{ Race } \\
\hline Amer Indian or Alaskan Native & $3(13)$ & & $2(4)$ & & $3(5)$ & & $3(4)$ & \\
\hline Asian American & $8(39)$ & & $10(17)$ & & $9(16)$ & & $4(6)$ & \\
\hline Black or African American & $14(70)$ & & $15(26)$ & & $14(25)$ & & $13(19)$ & \\
\hline Hawaiian or Pacific Islander & $0(1)$ & & $0(0)$ & & $0(0)$ & & $1(1)$ & \\
\hline White or Caucasian & $54(268)$ & & $56(99)$ & & $51(91)$ & & $54(78)$ & \\
\hline County-level & & $.000(.85)$ & & $-.05(.87)$ & & $.07(.81)$ & & $-.03(.86)$ \\
\hline Total & & $.000(.81)$ & & $-.04(.85)$ & & $.00(.80)$ & & $.05(.78)$ \\
\hline \multicolumn{9}{|l|}{ Interpersonal Stigma T1 } \\
\hline $\begin{array}{r}\text { Physically harmed } \\
\text { Phello }\end{array}$ & $12(62)$ & & $14(25)$ & & $10(17)$ & & $14(20)$ & \\
\hline Harassed (on or offline) & $49(244)$ & & $53(93)$ & & $46(83)$ & & $48(68)$ & \\
\hline Composite Scale & & $.61(.69)$ & & $.66(.69)$ & & $.56(.66)$ & & $.62(.70)$ \\
\hline \multicolumn{9}{|l|}{ Interpersonal Stigma $T 2 *$} \\
\hline Physically harmed & $14(56)$ & & $14(19)$ & & $14(20)$ & & $15(17)$ & \\
\hline Harassed (on or offline) & $47(188)$ & & $52(73)$ & & $41(60)$ & & $49(55)$ & \\
\hline Composite Scale & & $.61(.68)$ & & $.65(.69)$ & & $.55(.67)$ & & $.64(.69)$ \\
\hline
\end{tabular}

*Statistics for interpersonal stigma at T2 are based on the followed sample (N=400; Control PNF $n=141$; Alcohol PNF $n=146$; Alcohol+Coping PNF $n=113$ ).

There were no significant between-condition differences on any variables. 
Table 5

Perceived drinking norms and alcohol consumption pre-intervention (T1) and two months following receipt of treatment (T2).

\begin{tabular}{|c|c|c|c|c|}
\hline & $\begin{array}{c}\text { Overall } \\
\mathrm{T} 1 N=499 ; \mathrm{T} 2 N=400\end{array}$ & $\begin{array}{c}\text { Control PNF } \\
\text { T1 } n=177 ; \mathrm{T} 2 n=141\end{array}$ & $\begin{array}{c}\text { Alcohol PNF }{ }^{\mathrm{b}} \\
\text { T1 } n=179 ; \mathrm{T} 2 n=146\end{array}$ & $\begin{array}{c}\text { Alcohol+Coping PNF } \\
\text { T1 } n=143 \text {; T2 } n=113\end{array}$ \\
\hline & $M(S D)$ & $M(S D)$ & $M(S D)$ & $M(S D)$ \\
\hline \multicolumn{5}{|l|}{ T1 Perceived Drinking Norms } \\
\hline drinking days per week & $3.35(1.18)$ & $3.26(1.12)$ & $3.36(1.26)$ & $3.46(1.14)$ \\
\hline average drinks per occasion & $3.97(1.71)$ & $3.94(1.79)$ & $4.00(1.67)$ & $3.97(1.68)$ \\
\hline estimated weekly drinks (days*average) & $13.94(9.37)$ & $13.84(9.44)$ & $14.07(10.35)$ & $13.89(7.92)$ \\
\hline peak drinks on one occasion & $6.31(2.18)$ & $6.16(2.27)$ & $6.36(2.07)$ & $6.43(2.20)$ \\
\hline negative consequences experienced & $2.88(1.74)$ & $2.84(1.65)$ & $3.01(1.84)$ & $2.73(1.72)$ \\
\hline Z-norms composite & $-.03(.70)$ & $-.07(.71)$ & $.00(.75)$ & $-.01(.63)$ \\
\hline \multicolumn{5}{|l|}{ T1 Alcohol Use } \\
\hline drinking days per week & $2.86(1.50)$ & $2.65(1.41)$ & $2.56(1.42)$ & $2.85(1.51)$ \\
\hline average drinks per occasion & $3.26(1.58)$ & $3.33(1.52)$ & $3.15(1.73)$ & $3.30(1.47)$ \\
\hline estimated weekly drinks (days*average) & $9.15(7.51)$ & $9.13(7.90)$ & $8.96(8.19)$ & $9.43(6.00)$ \\
\hline peak drinks on one occasion & $5.79(2.34)$ & $5.74(2.43)$ & $5.76(2.37)$ & $5.87(2.19)$ \\
\hline negative consequences experienced & $2.52(1.89)$ & $2.45(1.95)$ & $2.55(1.86)$ & $2.58(1.89)$ \\
\hline Z-alcohol use composite & $-.02(.75)$ & $-.03(.75)$ & $-.06(.81)$ & $.03(.67)$ \\
\hline \multicolumn{5}{|l|}{ T2 Perceived Drinking Norms } \\
\hline drinking days per week & $2.60(.94)$ & $3.00(.95)^{* * * a, b}$ & $2.31(.71) * * * \mathrm{c}$ & $2.45(.59) * * * c$ \\
\hline average drinks per occasion & $2.89(1.13)$ & $3.52(1.03)^{* * * a, b}$ & $2.53(.67)^{* * * c}$ & $2.58(.72) * * * c$ \\
\hline estimated weekly drinks (days*average) & $7.73(4.24)$ & $10.77(4.98) * * * a, b$ & $5.90(2.76)^{* * * c}$ & $6.29(2.27) * * * c$ \\
\hline peak drinks on one occasion & $4.92(1.59)$ & $6.14(1.74)^{* * * a, b}$ & $4.32(.98) * * * c$ & $4.18(1.01)^{* * * c}$ \\
\hline negative consequences experienced & $2.76(1.22)$ & $3.55(1.26)^{* * * a, b}$ & $2.41(.98) * * * c$ & $2.21(.90)^{* * * c}$ \\
\hline Z-norms composite & $.00(.75)$ & $.64(.76) * * * a, b$ & $-.34(.48) * * * c$ & $-.35(.44) * * * c$ \\
\hline \multicolumn{5}{|l|}{ T2 Alcohol Use } \\
\hline drinking days per week & $2.48(1.29)$ & $2.72(1.33) * \mathrm{~b}$ & $2.32(1.32)^{* c}$ & $2.42(1.17)$ \\
\hline average drinks per occasion & $2.93(1.41)$ & $3.35(1.35)^{* * * a, b}$ & $2.72(1.29) * * * c$ & $2.68(.98) * * * \mathrm{c}$ \\
\hline estimated weekly drinks (days*average) & $8.44(7.52)$ & $9.73(6.83)^{* * * a, b}$ & $6.97(6.07)^{* * * c}$ & $6.66(4.13) * * * c$ \\
\hline peak drinks on one occasion & $4.90(2.23)$ & $5.91(2.30)^{* * * a, b}$ & $4.34(2.16)^{* * * c}$ & $4.35(1.71)^{* * * c}$ \\
\hline negative consequences experienced & $2.63(1.56)$ & $3.21(1.66)^{* * * a, b}$ & $2.29(1.51)^{* * * c}$ & $2.34(1.27) * * * \mathrm{c}$ \\
\hline Z-alcohol use composite & $.00(.79)$ & $.33(.85)^{* * * a, b}$ & $-.19(.79) * * * c$ & $-.17(.57) * * * c$ \\
\hline
\end{tabular}

Note. Significant between-condition differences are flagged with asterisks. Super-scripts ${ }^{\mathrm{a}, \mathrm{b}, \mathrm{c}}$ indicate pairs of conditions that differed significantly.

${ }^{*} p<.05 ;{ }^{* *} p<.01 ; * * * p<.001$. 


\section{Demographic Differences in Drinking, Norms, and Stigma Variables at Baseline.}

Potential baseline differences in alcohol and stigma-related variables by sexual identity, relationship status, and race/ethnicity were tested via one-way ANOVAs. Although recent survey studies have documented heavier drinking among bisexual women compared to lesbian women (e.g., Lewis, Ehlke, Shappie, Braitman, \& Heron, 2019; Schuler \& Collins, 2020), in this study sexual identity was not significantly associated with alcohol use, $F(2,496)=.93, p=.39$, or perceptions of drinking norms, $F(2,496)=1.01, p=.36$. Sexual identity was also unrelated to severe interpersonal stigma exposure at baseline, $F(2,496)=1.08, p=.34$, and the county-level measure of structural stigma, $F(2,496)=.43, p=.65$. However, state-level structural stigma did significantly differ by sexual identity, $F(2,496)=3.96, p=.02$. Relative to both bisexual $(M=.12 ; S D=.86)$ and lesbian women $(M=.02 ; S D=.89)$, queer-identified women $(M=-.12$; $S D=.86)$ resided in states with less structural stigma, $p \mathrm{~s}<.03$.

Consistent with previous findings (e.g., Veldhuis et al., 2019), single SMW (M=.16; $S D=.85)$ consumed more alcohol at baseline than partnered SMW $(M=-.12 ; S D=.70), F(1,497)$ $=15.87, p<.001$. Single participants $(M=.08 S D=.74)$ also perceived same sexual identity peers to consume more alcohol than partnered participants $(M=-.06 ; S D=.67), F(1,497)=4.77, p$ $<.03$. However, relationship status was not associated with severe interpersonal stigma exposure, $F(1,497)=1.07, p=.30$, nor structural stigma at the state, $F(1,497)=.01, p=.90$, or countylevel, $F(2,496)=.82, p<.36$.

When race was examined as a categorical variable with seven levels, there were no significant differences between racial groups in alcohol use, perceptions of drinking norms, interpersonal stigma exposure, or measures of structural stigma. However, because the racial category numbers for several minority groups were very small, additional analyses compared all 
racial/ethnic minority SMW to non-Hispanic white SMW on these variables. At baseline, racial/ethnic minority SMW $(M=.07 ; S D=.76)$ consumed more alcohol than non-Hispanic white $\operatorname{SMW}(M=-.07 S D=.78), F(1,497)=4.28, p=.03$. Racial/ethnic minority SMW $(M=.06$ $S D=.75)$ also perceived fellow SMW in their sexual identity and age-group to consume more alcohol than non-Hispanic white SMW $(M=-.06 S D=.65), F(1,497)=4.18, p=.04$. However, these groups did not differ on severe interpersonal stigma exposure, $F(1,497)=2.59, p=.11$, or structural stigma at the state, $F(1,497)=.001, p=.99$, or county-level, $F(1,497)=.408, p=.52$. Consistent with previous findings (e.g., Boyle et al., 2016), age in years was negatively correlated with alcohol consumption $(r=-.17, p<.001)$ and perceiving fellow SMW of the same sexual identity and age to consume less alcohol $(r=-.20, p<.001)$. Older age was also associated with decreased severe interpersonal exposure at baseline $(r=-.14, p=.002)$ but was unrelated to structural stigma $(r=-.06, p=.16)$. Table 6 presents bivariate and point-biserial correlations between dummy coded categorical demographic characteristics, continuous demographic characteristics, stigma variables, and perceived alcohol use norms and behaviors at baseline. 
Table 6

Correlations between perceived drinking norms, alcohol consumption, stigma variables, and demographic characteristics at baseline ( $n=499)$

\begin{tabular}{|c|c|c|c|c|c|c|c|c|c|c|}
\hline & 1 & 2 & 3 & 4 & 5 & 6 & 7 & 8 & 9 & 10 \\
\hline 1. Drinking Norms & - & $.60 * * *$ & $.16 * * *$ & $.10^{*}$ & $.10^{*}$ & -.04 & -.04 & .07 & $.09 *$ & $-.20 * * *$ \\
\hline 2. Alcohol Use & & - & $.22 * * *$ & .05 & $.18 * * *$ & .01 & -.06 & .05 & $.09 *$ & $-.17 * * *$ \\
\hline 3. Interpersonal Stigma & & & - & .07 & .05 & .01 & .06 & -.05 & -.07 & $-.14 * *$ \\
\hline 4. Total Structural Stigma & & & & - & .02 & .05 & $-.09 *$ & .05 & -.01 & -.06 \\
\hline 5. Single Relationship Status (coded 1) & & & & & - & .03 & .05 & -.06 & $.17 * * *$ & $-.08 *$ \\
\hline 6. Bisexual Identity (coded 1) & & & & & & - & $.26 * * *$ & $.63 * * *$ & -.03 & -.04 \\
\hline 7. Queer Identity (coded 1) & & & & & & & - & $.56 * * *$ & -.02 & -.07 \\
\hline 8. Lesbian Identity (coded 1$)$ & & & & & & & & - & .05 & $.10^{*}$ \\
\hline 9. Racial/ethnic minority status (coded 1 ) & & & & & & & & & - & $.11 *$ \\
\hline 10. Age in years & & & & & & & & & & 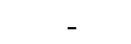 \\
\hline
\end{tabular}

Table 7

Supplemental baseline correlations including individual drinking norm and alcohol use items as well as total, state, and county-level structural stigma measures $(n=499)$

\begin{tabular}{|c|c|c|c|c|c|c|c|c|c|c|c|c|}
\hline & 1 & 2 & 3 & 4 & 5 & 6 & 7 & 8 & 9 & 10 & 11 & 12 \\
\hline 1. Norm-drinking days & - & $.18 * * *$ & $.23 * * *$ & $.33 * * *$ & $.44 * * *$ & $.23 * * *$ & $.23 * * *$ & $.28 * * *$ & .06 & .01 & .01 & .01 \\
\hline 2. Norm-average drinks & & - & $.51 * * *$ & $.35 * * *$ & .01 & $.49 * * *$ & $.41 * * *$ & $.30 * * *$ & $.10 *$ & $.11 *$ & .08 & $.11 *$ \\
\hline 3. Norm-peak drinks & & & - & $.38 * * *$ & $.13 * * *$ & $.44 * * *$ & $.53 * * *$ & $.36 * * *$ & $.10 *$ & $.11 *$ & $.12 *$ & .07 \\
\hline 4. Norm-consequences & & & & - & $.25 * * *$ & $.32 * * *$ & $.33 * * *$ & $.60 * * *$ & $.18 * * *$ & .07 & .03 & $.10 *$ \\
\hline 5. Own-drinking days & & & & & - & $.23 * * *$ & $.34 * * *$ & $.40 * * *$ & $.15 * *$ & .03 & .03 & .02 \\
\hline 6. Own-average drinks & & & & & & - & $.76^{* * * *}$ & $.53 * * *$ & $.13^{* *}$ & .02 & -.01 & .04 \\
\hline 7. Own-peak drinks & & & & & & & - & $.57 * * *$ & $.16 * *$ & .01 & .04 & -.01 \\
\hline 8. Own-consequences & & & & & & & & - & $.25 * * *$ & $.09 *$ & .07 & $.10^{*}$ \\
\hline 9. Interpersonal Stigma & & & & & & & & & - & .07 & .05 & .08 \\
\hline 10. Total Structural Stigma & & & & & & & & & & - & $.92 * * *$ & $.79 * * *$ \\
\hline 11. Structural Stigma-State & & & & & & & & & & & - & $.58 * * *$ \\
\hline 12. Structural Stigma-County & & & & & & & & & & & & - \\
\hline
\end{tabular}

$* p<.05 ; * * p<.01 ; * * * p<.00$ 


\section{Relationships Between Baseline Stigma Variables, Drinking Norms, and Alcohol Use.}

Consistent with research framing interpersonal and structural forms of sexual minority stigma as distinct constructs (e.g., Hatzenbuehler, 2016), interpersonal stigma exposure at baseline was not significantly correlated with total, state-level, or county-level measures of structural stigma (all $r \mathrm{~s}>.08$, all $p \mathrm{~s}>.05$ ). Also consistent with a large body of sexual minority stress research (see Hughes et al., 2020 for a review), greater interpersonal stigma exposure was significantly associated with heavier drinking at baseline $(r=.22, p<.001)$. Less expected, however, was the significant relationship between interpersonal stigma exposure and perceived drinking norms (Table $6 ; r=.16, p<.001$ ). Further, the total measure of structural stigma was significantly correlated with perceived drinking norms $(r=.10, p=.03)$ but not alcohol use at baseline and individual measures of state- and county-level structural stigma measures more consistently shared significant relationships with individual drinking norms than alcohol use items.

Whereas this study's stigma moderation hypotheses centered on the prediction that stigma experiences, as sources of outgroup threat, would strengthen the relationship between perceived sexual identity specific drinking norms and drinking behavior, the correlations observed suggest that these forms of sexual minority stigma may also be associated with perceptions of LBQ peers' drinking more frequently and consuming greater quantities of alcohol. Although temporal relationships are impossible to tease apart with cross-sectional data, an explanation for this pattern of correlations consistent with previous research is greater sexual minority stigma exposure is related to heavier drinking among LBQ women (e.g., Hughes et al., 2020), which in turn, is related to elevated perceptions of peers' drinking (i.e., false consensus; Miller \& Prentice, 2016). However, this type of explanation does not hold for structural stigma, 
as greater structural stigma was associated with elevated perceptions of LBQ drinking norms but not participants' own drinking. As higher structural stigma scores reflected lacking LGBTQ community resources and a decreased density of same-sex couples, one plausible explanation is that SMW residing in these areas have lesser opportunities to observe the drinking of LBQ peers and drink themselves in LGBTQ bar and nightclub settings. With LBQ peers less visible locally, it is possible that these participants' estimates of peer drinking norms may be heavily influenced by popular film and television-based depictions of LBQ women residing in liberal, queer enclaves and frequently drinking in LGBTQ bars and clubs. Although beyond the scope of the current study, the potential for such a media effect on perceptions of LBQ norms is considered more broadly in Chapter 6 as a direction for future research.

To better understand the interplay between these constructs and inform the interpretation of later tests of moderation (H3 \& H4), a final set of preliminary analyses tested whether stigma variables cross-sectionally moderated the relationship between perceived drinking norms and alcohol use at baseline. In concurrent models predicting baseline drinking, initial steps included demographic covariates, then perceived drinking norms and respective stigma-related variables were added in second steps, and then respective stigma*norms interaction terms were added in the final steps. These results are summarized in Table 8. 
Table 8

Summary of hierarchical regression models testing sexual minority stigma variables as moderators of the relationship between perceived drinking norms and alcohol use at baseline $(N=499)$.

\begin{tabular}{|c|c|c|c|c|c|c|}
\hline Model, Step & Predictor & $B$ & $S E$ & $t$ & $F \Delta$ & $R^{2} \Delta$ \\
\hline 1,1 & Queer sexual identity & -.17 & .09 & -1.83 & $6.31 *$ & .07 \\
\hline 1,1 & Bisexual sexual identity & -.05 & .08 & -.63 & & \\
\hline 1,1 & Racial/ethnic minority status & .13 & .07 & 1.85 & & \\
\hline 1,1 & Age in years & $-.02 * * *$ & .005 & $-4.00 * * *$ & & \\
\hline 1,1 & Single relationship status & $.24 * * *$ & .07 & $3.44 * * *$ & & \\
\hline 1,1 & Total structural stigma & .02 & .03 & .65 & & \\
\hline 1,2 & z-Perceived drinking norms T1 & $.62 * * *$ & .04 & $15.54 * * *$ & $136.60 * * *$ & .33 \\
\hline 1,2 & Interpersonal stigma exposure $\mathrm{T} 1$ & $.14^{* *}$ & .04 & $3.44 * *$ & & \\
\hline 1,3 & T1 interpersonal stigma*T1 norms & $.12 *$ & .05 & $2.14 *$ & $4.51 *$ & .01 \\
\hline 2,1 & Queer sexual identity & $-.19 *$ & .08 & $-2.17 *$ & 10.13 & .11 \\
\hline 2,1 & Bisexual sexual identity & -.06 & .08 & -.67 & & \\
\hline 2,1 & Racial/ethnic minority status & $.15^{*}$ & .07 & $2.17 *$ & & \\
\hline 2,1 & Age in years & $-.02 * * *$ & .004 & $-3.55 * * *$ & & \\
\hline 2,1 & Single relationship status & $.22 * *$ & .07 & $3.31 * *$ & & \\
\hline 2,1 & Interpersonal stigma exposure $\mathrm{T} 1$ & $.23^{* *}$ & .05 & $4.66^{* *}$ & & \\
\hline 2,2 & z-Perceived drinking norms T1 & $.62 * * *$ & .04 & $15.54 * * *$ & $120.75 * * *$ & .29 \\
\hline 2,2 & County-level structural stigma & -.03 & .03 & -.81 & & \\
\hline 2,3 & County-structural stigma* T1 norms & -.01 & .05 & -.17 & .03 & $<.001$ \\
\hline 3,1 & Queer sexual identity & $-.19 *$ & .08 & $-2.17 *$ & $10.13 * * *$ & .11 \\
\hline 3,1 & Bisexual sexual identity & -.06 & .08 & -.66 & & \\
\hline 3,1 & Racial/ethnic minority status & $.15^{*}$ & .07 & $2.17 *$ & & \\
\hline 3,1 & Age in years & $-.02 * * *$ & .005 & $-3.54 * * *$ & & \\
\hline 3,1 & Single relationship status & $.23 * *$ & .07 & 3.31 & & \\
\hline 3,1 & Interpersonal stigma exposure $\mathrm{T} 1$ & $.23^{* *}$ & .05 & $4.66 * *$ & & \\
\hline 3,2 & $\mathrm{z}$-Perceived drinking norms $\mathrm{T} 1$ & $.62 * * *$ & .04 & $15.51 * * *$ & $120.42 * * *$ & .29 \\
\hline 3,2 & State-level structural stigma & -.02 & .03 & -.46 & & \\
\hline 3,3 & State-structural stigma ${ }^{*} \mathrm{~T} 1$ norms & .03 & .04 & .68 & .46 & $<.001$ \\
\hline
\end{tabular}

Coefficients are presented for the steps in which predictors first entered the model $* p<.05 ; * * p<.01 ; * * * p<.001$

There was a significant interaction between interpersonal stigma exposure and perceived drinking norms predicting alcohol use at baseline $(B=.12, S E=.05, p=.02)$. This interaction, plotted in Figure 5, demonstrates that greater interpersonal stigma exposure strengthened the relationship between perceived drinking norms and drinking behavior, consistent with predictions and previous cross-sectional findings with gay men (Hamilton \& Mihalik, 2008). In 
subsequent models, however, neither of the interaction terms crossing structural stigma measures with perceived norms reached significance.

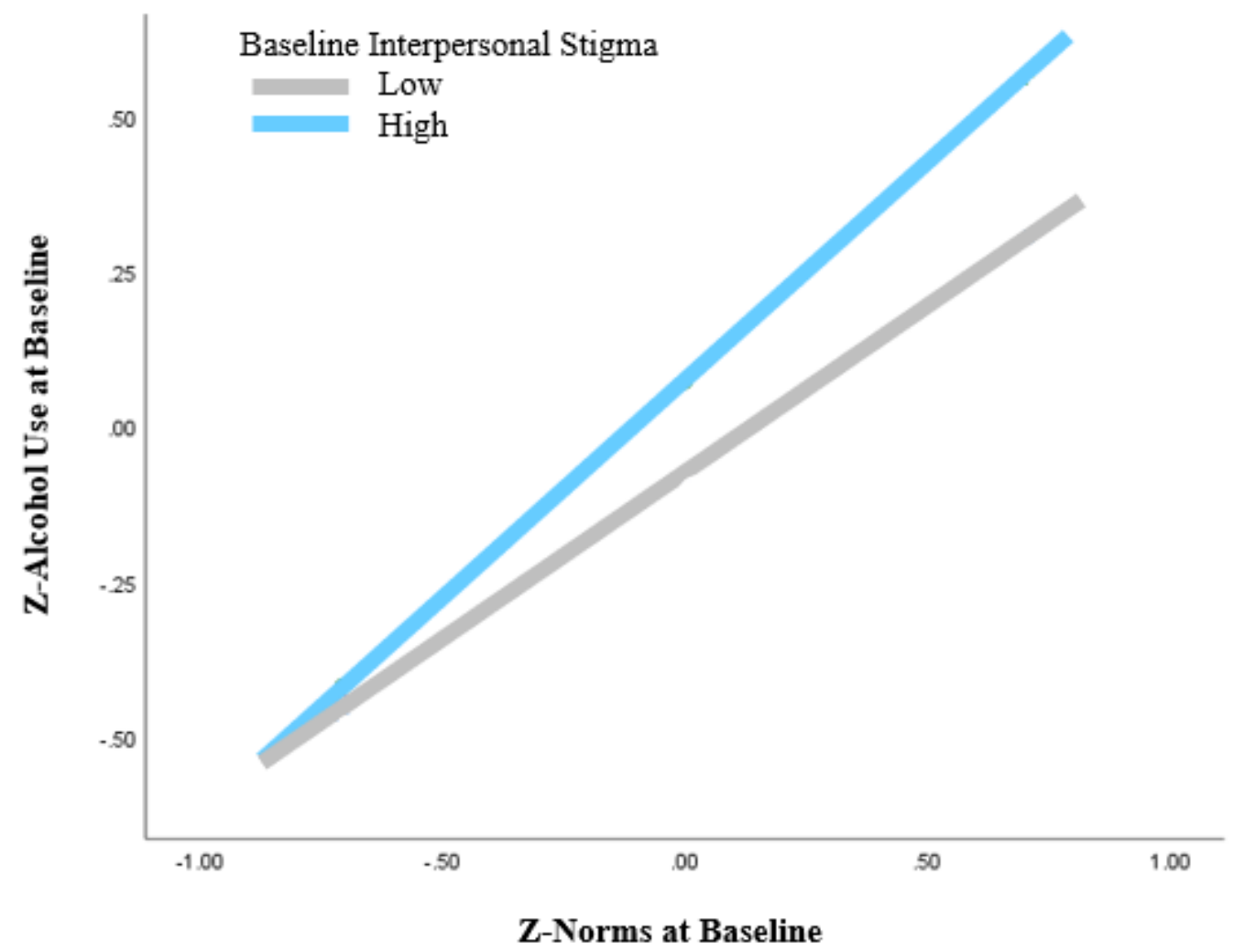

Figure 5. The relationship between perceived sexual identity specific drinking norms at low (-1 $S D$ from the mean) and high (+1 SD from the mean) interpersonal stigma at baseline

\subsection{Main Effects}

\section{Analytic Approach}

$\mathrm{H} 1$ and $\mathrm{H} 2$ were addressed through a two-step approach that first examined the main effects of PNF condition on respective composite measures of perceived alcohol use norms and alcohol use behaviors at follow-up. In the presence of significant conditional effects on the composite alcohol use outcome, parallel models then individually examined conditional effects on three clinically significant alcohol use outcomes: estimated drinks per week, peak drinks on one occasion, and number of negative alcohol-related consequences experienced. 
The composite alcohol use outcome was roughly normally distributed with slight positive skew, whereas all individual alcohol use outcomes were non-normally distributed count variables (i.e., number of drinks consumed weekly and on peak drinking occasion, number of negative consequences). For consistency, $\mathrm{H} 1$ and $\mathrm{H} 2$ were tested across outcomes via generalized linear models (GLMM) with robust standard errors (i.e., Huber-White sandwich estimator) requested to adjust for heteroscedasticity and the optimal distribution (e.g., linear, negative binomial, Poisson) specified for each outcome. For count outcomes estimated drinks per week, peak drinks on one occasion, and negative consequences experienced, dispersion was examined to determine whether GLMM should specify negative binomial or Poisson distributions. The estimated drinks per week outcome yielded a chi-square/df deviance statistic greater than 1, indicating that negative binomial distribution specification was optimal for the model predicting this outcome. In contrast, peak drinks on one occasion and number of negative alcohol-related consequences produced deviance statistics less than 1 suggesting that Poisson specification was more appropriate (Hilbe, 2014; Atkins et al., 2013). All GLMM models examined relationships between study condition and each outcome at follow-up while holding constant participants' sexual identity, age, racial/ethnic minority status, relationship status, total interpersonal stigma exposure during the study period, total structural stigma exposure, and the baseline measure of the outcome. In the presence of significant overall model effects for categorical variables with three or more levels (e.g., study condition, sexual identity), pairwise comparisons of marginal means were requested. 


\section{Complete Case Tests of Main Effect Hypotheses 1 \& 2}

Alcohol Use Composite. The linear-specified GLMM (Table 9) revealed significant model effects for the composite measure of alcohol use at baseline, Wald $X^{2}(1)=417.59 ; p<.001$, and study condition, Wald $\left.X^{2}(2)=87.25 ; p<.001\right)$. The positive coefficient for baseline alcohol use $(B=.75, S E=.04, p<.001)$ indicated that greater drinking at baseline was associated with greater drinking at follow-up when study condition and other variables were held constant. This finding is consistent with the larger PNF literature (e.g., Miller \& Prentice, 2016), which explains that in response to alcohol PNF drinkers tend to adjust their consumption from preintervention levels rather than fully augmenting their drinking to match the normative standard presented. Thus, a positive relationship between alcohol use at baseline and follow-up was expected across study conditions. Offering support for $\mathrm{H} 1$, the negative coefficients for

Table 9

Linear GLMM results examining the impact of covariates and study condition on the composite measure of alcohol use at follow-up.

\begin{tabular}{|c|c|c|c|c|c|c|}
\hline Predictor & $B$ & $S E$ & Wald & $R R$ & $\begin{array}{c}95 \% \mathrm{CI} \\
{[R R]}\end{array}$ & $\begin{array}{c}\text { Marginal Mean } \\
\text { Comparisons } \\
M(S E)\end{array}$ \\
\hline Racial/Ethnic Minority (dummy) & -.006 & .05 & .01 & .99 & $.89-1.10$ & \\
\hline Single Rel Status (dummy) & .05 & .06 & .68 & 1.05 & $.94-1.17$ & \\
\hline Age in Years & .003 & .003 & .001 & 1.00 & $.99-1.00$ & \\
\hline Total Interpersonal Stigma & -.03 & .03 & 1.09 & .97 & $.93-1.02$ & \\
\hline Total Structural Stigma & .003 & .02 & .01 & 1.00 & $.95-1.06$ & \\
\hline T1 Alcohol Use Composite & $.75^{* * *}$ & .04 & 417.59 & 2.12 & $1.97-2.28$ & \\
\hline Sexual Identity & & & 4.53 & & & \\
\hline Queer $^{A}$ & -.03 & .07 & .19 & .97 & $.85-1.11$ & \\
\hline Bisexual $^{\mathrm{B}}$ & .12 & .06 & 3.49 & 1.13 & $.99-1.28$ & \\
\hline Lesbian (ref group) ${ }^{\mathrm{C}}$ & & & & & & \\
\hline Study Condition & & & $87.25 * * *$ & & & \\
\hline Alcohol+Coping PNF $^{\mathrm{A}}$ & $-.54 * * *$ & .06 & $74.82 * * *$ & .58 & $.51-.66$ & $-.19(.04)^{* * *} \mathrm{C}$ \\
\hline Alcohol PNF ${ }^{\mathrm{B}}$ & $-.51 * * *$ & .06 & $63.83 * * *$ & .60 & $.53-.68$ & $-.16(.04)^{* * * * C}$ \\
\hline Control PNF (ref group) ${ }^{\mathrm{C}}$ & & & & & & $.35(.05)^{* * * A, B}$ \\
\hline
\end{tabular}

Note: see Appendix E (Table E1) for the same analysis run as a linear regression model with study condition represented by dummy variables (for consistency with subsequent PROCESS-based moderator analyses). $* p<.05 ; * * p<.01 ; * * * p<.001$ 
treatment conditions revealed that PNF on alcohol use alone (H1a: $B=-.51, S E=.06, p<.001)$ and alcohol+coping $(\mathrm{H} 1 \mathrm{~b} ; B=-.54, S E=.06, p<.001)$ were associated with significant reductions in alcohol use at follow-up, relative to control PNF (the reference category). Note that GLMM parameters presented in Table 9 also include exponentiated coefficients known as rate ratios (RRs), which aid in understanding the degree to which treatment conditions reduced alcohol use. That is, RRs of 0.58 for alcohol only PNF and 0.60 for alcohol+coping PNF can be interpreted, respectively, as decreases of $42 \%$ and $40 \%$ in alcohol use relative to control PNF. Provided in the last column of Table 9 and mapping onto these RRs are the marginal means and standard errors derived for each study condition. Pairwise tests of the differences between these means are flagged for significance. Further supporting H1, mean composite alcohol use at follow-up in the control PNF condition $(M=.35, S E=.05)$ significantly exceeded means in both the alcohol+coping PNF condition $(M=-.19, S E=.04)$ and the alcohol only PNF condition $(M=-.16, S E=.04), p s$ $<.001$. Pairwise tests of the differences between conditional marginal means also provide a direct test of $\mathrm{H} 2$, which predicted that the alcohol+coping PNF condition would be associated with significantly less drinking at follow-up than the alcohol only PNF condition. Revealing an absence of support for $\mathrm{H} 2$, at follow-up, alcohol use in the alcohol+coping condition $(M=-.19$ $S E=.04)$ did not significantly differ from alcohol use in the alcohol only PNF condition $(M=-.16$ $S E=.04), p=.53$.

Estimated drinks per week, peak drinks, and negative consequences. As shown in Table 10, subsequent GLMM models predicting individual alcohol use outcomes at follow-up indicated effects similar in magnitude supporting $\mathrm{H} 1$ but failing to support H2. For instance, the negative binomial GLMM predicting estimated drinks per week indicated significant model effects for estimated weekly drinks at baseline (Wald $\left.x^{2}=159.00, p<.001\right)$ and PNF condition (Wald $x^{2}=$ 
$53.41, p<.001)$. Relative to control PNF, alcohol only PNF $(B=-.41, p<.001)$ and alcohol+coping PNF $(B=-.38, p<.001)$ were associated with significantly decreased drinks per week at follow-up, corresponding to reductions of $34 \%$ and $32 \%$, or roughly three drinks, respectively. However, suggesting an absence of support for $\mathrm{H} 2$, the alcohol+coping condition $(M=6.02 S E=.28)$ did not outperform the alcohol only PNF condition $(M=5.85 S E=.30)$ in reducing weekly drinks, $p=.67$.

Although significant model effects were largely consistent across outcomes (study condition, baseline measure of the alcohol outcome), divergence was observed in the significance of effects associated with one covariate. Specifically, in the model predicting peak drinks, there was a significant effect for sexual identity (Wald $x^{2}=9.20, p<.01$ ), not observed in the other models. Pairwise comparisons of marginal means revealed that at follow-up, bisexual participants $(M=5.08 S E=.18)$ consumed significantly more drinks on their heaviest drinking

Table 10.

GLMM results examining the impact of covariates and study condition on count alcohol outcomes at follow-up (continued on next page).

Estimated Weekly Drinks at Follow-Up (Negative Binomial-Specified GLMM)

\begin{tabular}{|c|c|c|c|c|c|c|}
\hline Predictor & $B$ & $S E$ & Wald & $R R$ & $\begin{array}{c}95 \% \mathrm{CI} \\
{[R R]}\end{array}$ & $\begin{array}{c}\text { Marginal } M(S E) \\
\text { Comparisons }\end{array}$ \\
\hline Racial/Ethnic Minority (dummy) & .02 & .05 & .08 & 1.02 & $.91-1.13$ & - \\
\hline Single Rel Status (dummy) & .08 & .06 & 1.78 & 1.08 & $.97-1.21$ & - \\
\hline Age in Years & -.003 & .004 & .64 & .99 & $.99-1.00$ & - \\
\hline Total Interpersonal Stigma & -.02 & .02 & .58 & 1.00 & $.99-1.00$ & - \\
\hline Total Structural Stigma & -.004 & .03 & .02 & .99 & $.94-1.03$ & - \\
\hline T1 Weekly Drinks & $.07 * * *$ & .006 & $159.01 * * *$ & 1.08 & $1.06-1.09$ & - \\
\hline Sexual Identity & & & 2.18 & & & - \\
\hline Queer $^{A}$ & -.02 & .07 & .09 & .98 & $.85-1.13$ & - \\
\hline $\begin{array}{r}\text { Bisexual }^{\mathrm{B}} \\
\text { Leshian (ref oroun) }^{\mathrm{C}}\end{array}$ & .08 & .06 & 1.64 & 1.08 & $.96-1.21$ & - \\
\hline $\begin{array}{l}\text { Lesbian (ref group) } \\
\text { Study Condition }\end{array}$ & & & $53.40 * * *$ & & & $\begin{array}{l}- \\
-\end{array}$ \\
\hline Alcohol+Coping PNF $^{\mathrm{A}}$ & $-.39 * * *$ & .06 & $37.87 * * *$ & .68 & $.60-.77$ & $6.00(.28)^{* * * \mathrm{C}}$ \\
\hline $\begin{array}{r}\text { Alcohol PNF } \\
\text { Control PNF (ref group) } \\
\text { C }^{\mathrm{B}}\end{array}$ & $-.42 * * *$ & .07 & $41.33 * * *$ & .66 & $.58-.75$ & $\begin{array}{c}5.83(.30) * * * \mathrm{C} \\
8.86(.40)^{* * * A, \mathrm{~B}}\end{array}$ \\
\hline
\end{tabular}


Peak Drinks at Follow-Up (Poisson-specified GLMM)

\begin{tabular}{|c|c|c|c|c|c|c|}
\hline Predictor & $B$ & $S E$ & Wald & $R R$ & $\begin{array}{c}95 \% \mathrm{CI} \\
{[R R]}\end{array}$ & $\begin{array}{c}\text { Marginal } M(S E) \\
\text { Comparisons }\end{array}$ \\
\hline Racial/Ethnic Minority (dummy) & .02 & .03 & .39 & 1.02 & $.96-1.10$ & - \\
\hline Single Rel Status (dummy) & .009 & .04 & .06 & 1.01 & $.94-1.08$ & - \\
\hline Age in Years & -.001 & .002 & .21 & 1.00 & $.99-1.00$ & - \\
\hline Total Interpersonal Stigma & .004 & .01 & .06 & 1.00 & $.98-1.03$ & - \\
\hline Total Structural Stigma & -.009 & .02 & .25 & .99 & $.96-1.03$ & - \\
\hline T1 Peak Drinks & $.11 * *$ & .006 & $241.23 * * *$ & 1.11 & $1.10-1.13$ & - \\
\hline Sexual Identity & & & $9.20 *$ & & & - \\
\hline Queer $^{A}$ & -.05 & .05 & .99 & .96 & $.87-1.04$ & $4.38(.17)^{* \mathrm{~B}}$ \\
\hline $\begin{array}{r}\text { Bisexual }^{\mathrm{B}} \\
\text { Lesbian (ref group) })^{\mathrm{C}}\end{array}$ & $.10^{*}$ & .04 & $6.20 *$ & 1.12 & $1.02-1.20$ & $\begin{array}{c}5.08(.18)^{* \mathrm{~A}, \mathrm{C}} \\
4.59(.10)^{* \mathrm{~B}}\end{array}$ \\
\hline Study Condition & & & $82.61 * * *$ & & & - \\
\hline Alcohol+Coping $\mathbf{P N F}^{\mathrm{A}}$ & $-.30 * * *$ & .04 & $59.71 * * *$ & .74 & $.68-.80$ & $4.21(.11)^{* * * \mathrm{C}}$ \\
\hline $\begin{array}{r}\text { Alcohol PNF }^{\mathrm{B}} \\
\text { Control PNF (ref group) }\end{array}$ & $-.31 * * *$ & .04 & $59.15 * * *$ & .73 & $.68-.79$ & $\begin{array}{c}4.18(.10) * * * \mathrm{C} \\
5.71(.12)^{* * * A, B}\end{array}$ \\
\hline
\end{tabular}

Number of Negative Consequences at Follow-up (Poisson-specified GLMM)

\begin{tabular}{|c|c|c|c|c|c|c|}
\hline Predictor & $B$ & $S E$ & Wald & $R R$ & $\begin{array}{c}95 \% \mathrm{CI} \\
{[R R]}\end{array}$ & $\begin{array}{c}\text { Marginal } M(S E) \\
\text { Comparisons }\end{array}$ \\
\hline Racial/Ethnic Minority (dummy) & .04 & .05 & .49 & 1.04 & $.94-1.15$ & - \\
\hline Single Rel Status (dummy) & -.02 & .05 & .10 & .98 & $.89-1.08$ & - \\
\hline Age in Years & -.003 & .003 & .90 & 1.00 & $.99-1.00$ & - \\
\hline Total Interpersonal Stigma & .004 & .02 & .03 & 1.00 & $.97-1.04$ & - \\
\hline Total Structural Stigma & .03 & .03 & 1.03 & 1.03 & $.98-1.08$ & - \\
\hline T1 Negative Consequences & $.14 * *$ & .01 & $120.51 * * *$ & 1.15 & $1.24-1.18$ & - \\
\hline Oueer $^{A}$ & 008 & & & 00 & $87 \quad 112$ & \\
\hline Lesbian (ref group) $^{\mathrm{C}}$ & $\begin{array}{l}-.000 \\
.09\end{array}$ & .06 & 2.33 & 1.09 & $.98-1.23$ & - \\
\hline $\begin{array}{r}\text { Study Condition } \\
\text { Alcohol+Coping } \text { PNF }^{\mathbf{A}} \\
\text { Alcohol PNF } \\
\text { B } \\
\text { Control PNF (ref group) }^{\mathbf{C}}\end{array}$ & $\begin{array}{l}-.33 * * * \\
-.35 * * *\end{array}$ & $\begin{array}{l}.06 \\
.06\end{array}$ & $\begin{array}{l}30.65 * * * \\
36.90 * * *\end{array}$ & $\begin{array}{l}.72 \\
.71\end{array}$ & $\begin{array}{l}.64-.81 \\
.63-.79\end{array}$ & $\begin{array}{c}2.24(.11)^{* * * \mathrm{C}} \\
2.20(.10)^{* * * \mathrm{C}} \\
3.12(.12)^{* * * \mathrm{~A}, \mathrm{~B}}\end{array}$ \\
\hline
\end{tabular}

$* p<.05 ; * * p<.01 ; * * * p<.001$

occasion than did both lesbian $(M=4.58, S E=.10)$ and queer-identified participants $(M=4.38$, $S E=.17), p s<.02$. It is worth noting that although sexual identity effects did not reach significance in the models predicting the other alcohol outcomes, in the model predicting composite alcohol use at follow-up, this effect trended toward significance (i.e., $p=.09$ ). As preliminary analyses 
did not reveal a significant relationship between sexual identity and alcohol use at baseline (bisexuals did not simply enter the study drinking more), this suggests that LezParlay treatment conditions may have been less effective in reducing alcohol use among bisexual participants, relative to lesbian and queer participants. Whether reductions in alcohol use associated with LezParlay treatment conditions varied as a function of sexual identity is directly examined later through exploratory moderator analyses.

\section{Comparison of ITT and CC Models \& Summary of H1 and H2 Findings}

Corresponding results from the more conservative ITT models with the full baseline sample $(N=499)$ are presented in Appendix E. In summary, ITT results paralleled those from CC models with significant reductions observed across alcohol outcomes (all $p s<.001$ ) in LezParlay's treatment PNF conditions relative to control (H1a and H1b supported) but no significant differences detected between treatment conditions (lack of support for $\mathrm{H} 2$ ). That is, across main effect models and outcomes there was no evidence that correcting norms for the stigma-coping behaviors engaged in by fellow SMW provided drinking reduction benefits beyond those associated with the correction of frequency and quantity drinking norms. However, it is possible that any additional benefits associated with the alcohol+coping PNF (relative to alcohol only PNF) are limited to specific sub-groups of participants. For instance, perhaps this condition outperforms alcohol only PNF only among participants with greater exposure to stigma (i.,e, H3b, H4b), those who entered the study as heavier drinkers (H6b), or racial/ethnic minority participants (exploratory demographic moderator). Thus, potential explanations for the null finding for $\mathrm{H} 2$ will be narrowed through a subsequent examination of participant-level moderators. 
Given the lack of differences between treatment conditions, comparisons of effect sizes yielded by CC and ITT approaches focus on differences between each treatment PNF condition and control. Cohen's $d$ estimates were calculated using the standard formula, which assumes that groups have similar standard deviations and sample sizes. However, as the three PNF conditions had different sample sizes and standard deviations at follow-up and this was a pre-post design with an active control condition, an alternative estimate of $d$ is also presented. Morris's formula for $d$ (Lenhard \& Lenhard, 2016; Morris, 2008) was calculated by subtracting the mean pre-post change in the control group from the mean pre-post changes in the treatment groups then dividing by the pooled pretest standard deviation. This measure of effect is not biased in the case of interventions, which result in reduced variability in the treatment group relative to control (e.g., PNF).

As presented in Table 11, across CC and ITT models, effect size calculations indicated that relative to the control PNF condition, both of LezParlay's treatment conditions had moderate effects on the composite measure of alcohol use (i.e., estimates of $d$ ranged from. 49 on the low end to.66 on the high end).

Table 11. H1: Estimates of effect sizes associated with LezParlay treatment conditions (relative to control) on composite alcohol use at follow-up.

\begin{tabular}{rcc}
\hline & Cohen's $d$ & Morris's $d$ \\
\hline Alcohol only PNF (relative to control PNF) & & .56 \\
CC model $n=400$ & .64 & .49 \\
ITT model $n=499$ & .51 & .64 \\
Alcohol+coping PNF (relative to control PNF) & & .50 \\
CC model $n=400$ & .66 & .55 \\
ITT model $n=499$ &
\end{tabular}




\subsection{Tests of Moderation}

As a total of 10 potential moderators of intervention efficacy were to be tested, and the goal of these analyses was to ascertain the groups of participants for whom treatment PNF delivered by LezParlay was most and least effective overall, tests of moderation focused on the composite measure of alcohol-related behaviors at follow-up. This limited the number of models to be run $(10$ models relative to the 40 that would be required to test moderators on both composite and individual outcomes) and allowed tests of moderation with robust standard errors (i.e., Huber-White) to be conducted via linear regression using Hayes (2017) PROCESS macro (Model 1) for SPSS. As the composite measure of alcohol use was roughly normally distributed, this linear regression approach is highly similar to the GLMM approach taken in Table 8 (see the parallel linear regression-based results for $\mathrm{H} 1$ and $\mathrm{H} 2$ obtained for composite alcohol use at follow-up in Table F1 of Appendix F) but requires categorical variables to be fully represented by dummy variables with one level of each categorical variable designated as a reference group and omitted from the model. Whereas interactions between categorical and continuous variables are difficult to probe via GLMM, PROCESS allows significant interactions between variables of all types to be probed via tests of conditional effects (Hayes, 2017), making it the preferable strategy for testing the current trial's moderator-based hypotheses. For all moderator-related hypotheses and exploratory questions, in-text presentation of results are limited to CC tests of interaction terms (coefficients, significance, unique contribution to variability) with significant interactions examined via tests of conditional effects for continuous moderators and GLMM for categorical moderators. However, full CC model results, including coefficients for all covariates, are provided in Appendix E. 


\section{Tests of Interpersonal \& Structural Stigma as Moderators of Efficacy (H3-H5)}

To address H3-H4, separate models tested time-specific exposure to interpersonal stigma as well as state and county-level measures of structural stigma as potential moderators of relationships between PNF condition and the alcohol-related behaviors composite at follow-up. In separate models featuring each stigma-related measure, two-way interaction terms were computed by crossing the potential moderator with dummy variables comparing each intervention condition to control $(\mathrm{H} 3 \mathrm{a}, \mathrm{H} 4 \mathrm{a})$ and then each intervention condition to the other intervention condition (H3a, H4b). Covariates in each model included baseline alcohol use, sexual identity, age, race/ethnicity, and relationship status. In addition, as the measures of structural and interpersonal stigma were not significantly correlated with one another but individual measures (i.e., T1 \& T2 interpersonal stigma; county and state-level structural stigma) within each form were (see Tables $6 \& 7$ ), models also controlled for the total measure of the competing form of stigma (i.e., total interpersonal stigma was controlled for in models testing level-specific structural stigma-related moderators, total structural stigma controlled for in models testing time-specific interpersonal stigma-related moderators). This was done to control for the competing form of stigma in models while avoiding the multi-collinearity that would be introduced by including correlated individual time/level-specific stigma measures.

Figure 6 provides a summary of the timing of stigma-related assessments in this study and the periods of time in which they covered. As interpersonal stigma is acutely experienced, this was assessed at baseline and follow-up, with items at both time-points inquiring as to whether participants were physically harmed or verbally harassed due to sexual minority status during the previous 2-month period. In contrast, structural stigma is location-based and chronic. 
Therefore, total, state, and county-level structural stigma scores were consistent across the entire study period.

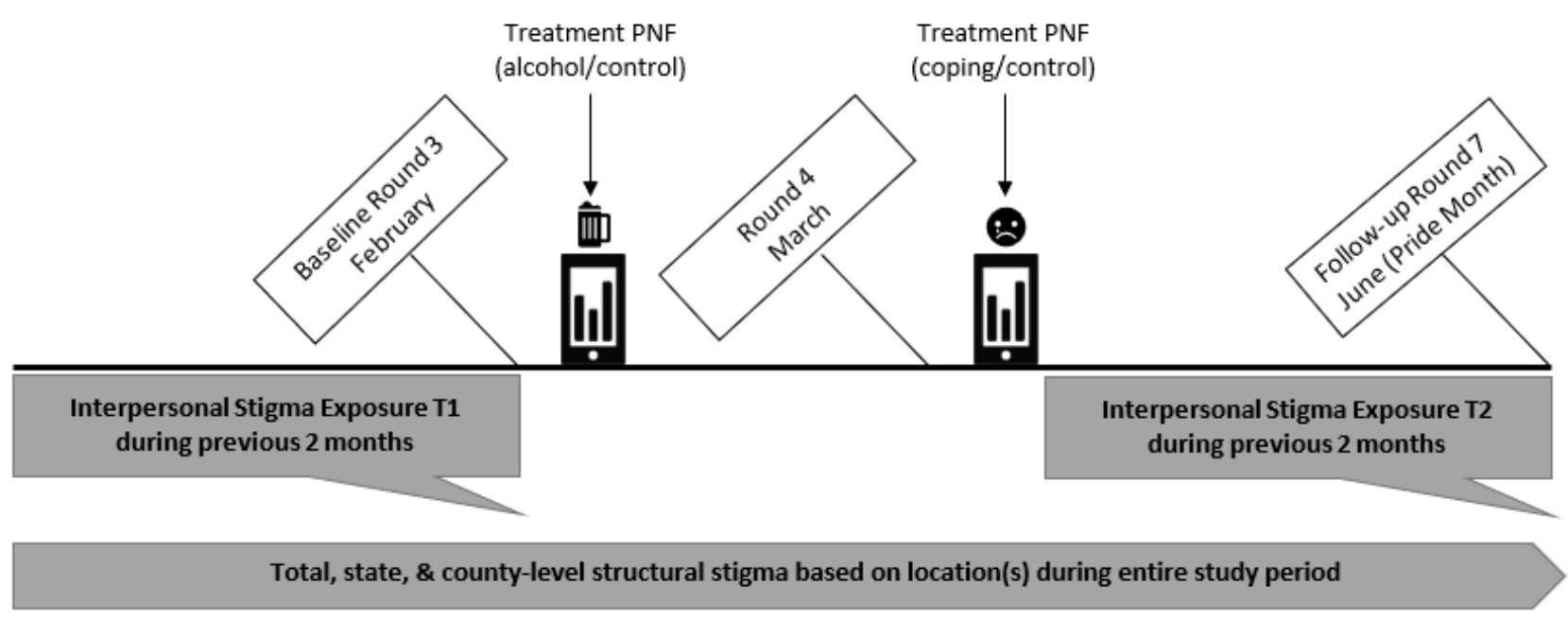

Figure 6. The timing of stigma-related assessments within the study.

Interpersonal Stigma Exposure H3. Given the acute nature of the interpersonal stigma experiences assessed, the $\mathrm{T} 1$ measure was expected to moderate the relationship between perceived drinking norms and alcohol use at baseline, which it did (see Figure 5 in preliminary analysis section). Meanwhile, the T2 measure was predicted to moderate the relationship between treatment PNF conditions and alcohol use at follow-up (H3a). The specificity of this hypothesis was first explored through a preliminary model that sought to confirm that the T1 measure of interpersonal stigma did not moderate intervention efficacy. As shown in the top of Table 12 (Model 1), the coefficients for interactions between interpersonal stigma exposure at T1 and treatment PNF conditions did not significantly differ from zero $(B=-.15, S E=.09, p=.11 ; B=$ $-.14, S E=.10, p=.16)$. Thus, as expected, historically experienced interpersonal stigma taking place during the months prior to PNF delivery did not significantly enhance (nor diminish) the 
impact of treatment PNF, although it did strengthen the relationship between perceived drinking norms and alcohol use at baseline.

Table 12

Summary of condition*stigma interactions in CC models predicting the composite measure of alcohol consumption at follow-up $(n=400)$

\begin{tabular}{cccccc}
\hline Predictor & $B$ & $S E$ & $t$ & $F^{\text {change }}$ & $R^{2 \text { change }}$ \\
\hline $\begin{array}{c}\text { Model 1: T1 Interpersonal Stigma as moderator } \\
\text { Alcohol+Coping PNF*T1 Interpersonal stigma }\end{array}$ & -.15 & .09 & -1.61 & & .003 \\
Alcohol PNF* T1 Interpersonal stigma & -.14 & .10 & -1.49 & & \\
Model 2: T2 Interpersonal Stigma as moderator & & & & 1.41 & \\
Alcohol+Coping PNF*T2 Interpersonal stigma &.$- .42^{* * * *}$ & .08 & $-5.18^{* * *}$ & & $.03 * * *$ \\
Alcohol PNF* T2 Interpersonal stigma & $-.38^{* * *}$ & .09 & $-4.63^{* * *}$ & & \\
\hline
\end{tabular}

See Appendix E, Table E2 for full PROCESS model results and covariate information. *** $p<.001$.

Model 2 (Table 12) then provided a direct test of H3a. Consistent with this hypothesis, interaction terms for interpersonal stigma exposure at T2*treatment PNF conditions interaction accounted for $3 \%$ the variability of alcohol use at follow-up, $F \Delta(2,387)=15.57, p<.001$, and the coefficients for both interaction terms significantly differed from zero. Conditional effects with $\pm 1 S D$ from the mean were used to probe the interactions. As shown in Figure 7, relative to the control PNF condition where drinking at follow-up increased as stigma exposure increased, in both treatment PNF conditions drinking at follow-up decreased as stigma exposure increased. Specifically, the alcohol+coping PNF condition was associated with reduced drinking when interpersonal stigma exposure was low (-1 SD from the mean) following PNF delivery $(B=-.30$, $S E=.06, p<.001)$, and when interpersonal stigma exposure was high (+1 $S D$ from the mean) following PNF delivery $(B=-.79, S E=.06, p<.001)$. Similarly, the alcohol only PNF condition predicted reduced drinking when interpersonal stigma exposure was low $(B=-.25, \mathrm{SE}=.08, p$ $=.003)$, as well as high $(B=-.78, S E=.08, p<.001)$. Thus, in support of H3a, LezParlay treatment 
PNF conditions were associated with reduced drinking at follow-up among SMW experiencing both low and high interpersonal stigma exposure in the months that followed PNF delivery; however, these relationships were stronger when interpersonal stigma exposure was high.

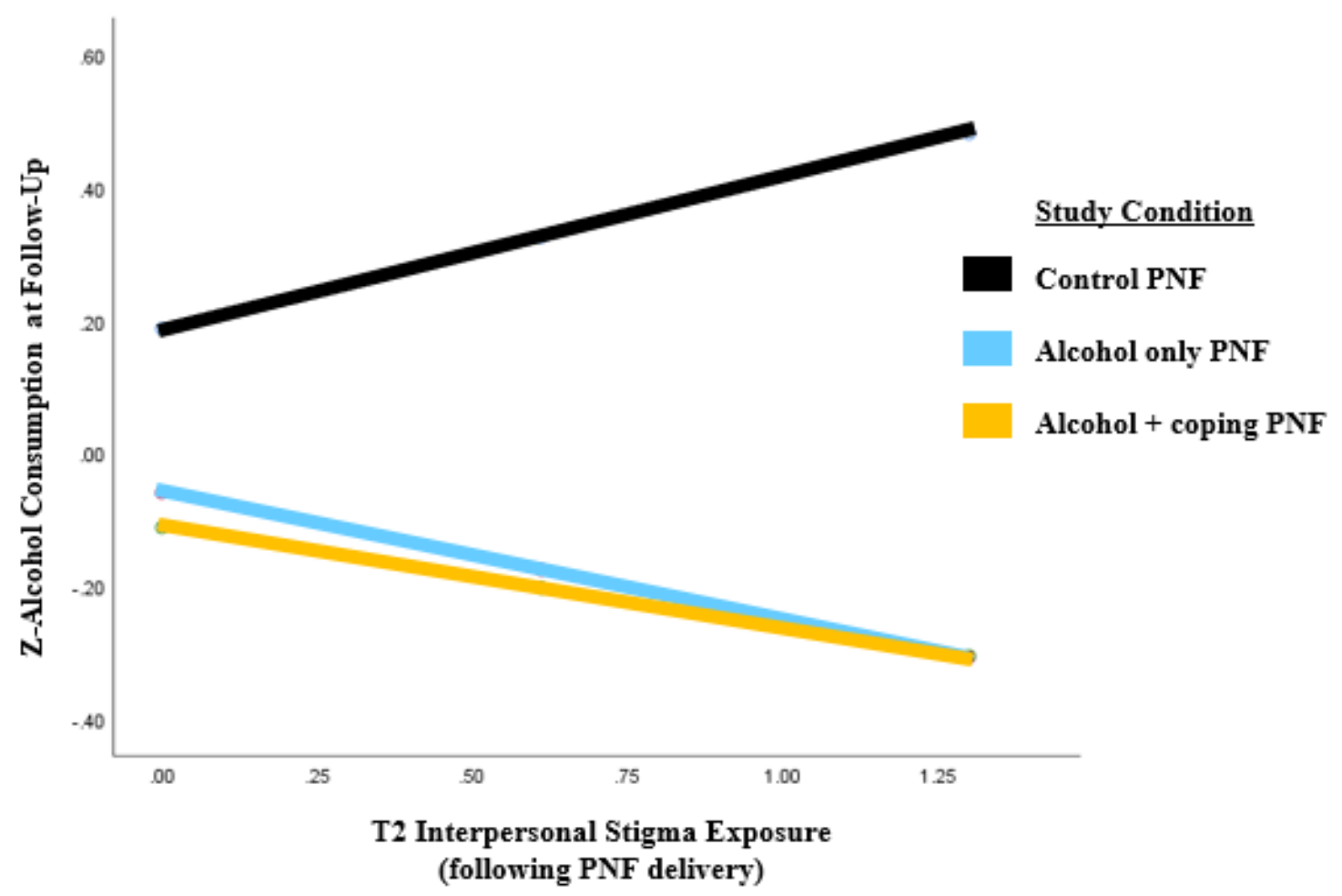

Figure 7. Alcohol use at follow-up as a function of study condition and interpersonal stigma exposure during the 2-month period following PNF delivery.

$\mathrm{H} 3 \mathrm{~b}$ further predicted that probing significant interactions would reveal that among participants reporting greater interpersonal stigma at T2, the alcohol+coping PNF condition would be associated with larger reductions in drinking than the alcohol PNF condition. The fact that the lines for treatment conditions plotted in Figure 7 appear to only differ minimally (and converge at high interpersonal stigma) suggests a lack of support for this hypothesis. To test this hypothesis formally, model 7 was re-run with dummy variables representing study condition shifted so that interaction terms included in the model featured alcohol only PNF and control 
PNF (alcohol+coping PNF specified as the reference group). Confirming a lack of support for $\mathrm{H} 3 \mathrm{~b}$, the alcohol only $\mathrm{PNF}^{*} \mathrm{~T} 2$ interpersonal stigma interaction term did not significantly differ from zero $(B=-.04, S E=.07, t=-.55, p=.58)$, indicating the two treatment conditions were not differentially effective in reducing alcohol use as a function of interpersonal stigma reported at $\mathrm{T} 2$.

\section{Total, State, County-Level Structural Stigma as Moderators (H4).}

Table 13 presents the non-significant interaction terms crossing structural stigma variables with study condition. Indicating a lack of support for $\mathrm{H} 4 \mathrm{a}$, adding the interaction terms to the models did not significantly increase the variability in alcohol use accounted for, and none of the interaction term coefficients significantly differed from zero (all $p s>.40$ ). Thus, there was no evidence that structural stigma experienced as a function of geographic location during the study period enhanced (nor diminished) the impact of treatment PNF relative to control.

\section{Table 13}

H4: Summary of condition*structural stigma interactions tested in CC PROCESS models predicting the composite measure of alcohol consumption at follow-up $(n=400)$

\begin{tabular}{cccccc}
\hline Predictor & $B$ & $S E$ & $t$ & $F \Delta$ & $R^{2} \Delta$ \\
\hline Model 3: Total Structural Stigma as moderator & & & & .45 & .001 \\
Alcohol+Coping PNF*Structural Stigma & -.07 & .08 & -.89 & & \\
Alcohol PNF*Structural Stigma & -.01 & .07 & -.18 & & \\
Model 4: County-Structural Stigma as moderator & & & & .60 & .001 \\
Alcohol+Coping PNF*County-Structural Stigma & -.07 & .07 & -1.00 & & \\
Alcohol PNF*County-Structural Stigma & -.001 & .07 & .35 & & $<.001$ \\
Model 5: State-Structural Stigma as moderator & & & & .35 & \\
Alcohol+Coping PNF*State Structural Stigma & -.06 & .07 & -.84 & & \\
Alcohol PNF*State Structural Stigma & -.03 & .06 & -.57 & &
\end{tabular}

See Appendix E, Table E3 for full PROCESS model results and covariate information. 
To formerly test H4b (i.e., that the alcohol+coping PNF condition would outperform the alcohol only PNF condition when structural stigma was high relative to low), the model was rerun with dummy variables shifted such that interaction terms in models featured the alcohol only PNF and control PNF conditions with alcohol+coping PNF condition witheld as the reference group. Confirming a lack of support for this hypothesis, non-significant coefficients for interaction terms in respective models crossing alcohol PNF with the three measures of structural stigma (*total: $B=.05, S E=.07, t=.76, p=.44$; *county-level: $B=.08, S E=.07, t=1.07, p=.28$; *statelevel: $B=.02, S E=.06, t=.30, p=.77)$ confirmed that the two treatment conditions were not differentially effective in reducing alcohol use as a function of structural stigma.

\section{Test of Baseline Drinking as a Moderator (H5)}

H5 predicted that the drinking reduction effects associated with both PNF conditions would be enhanced relative to control among participants who were heavier (relative to lighter) drinkers at baseline (H5a), and further, the alcohol+coping condition would better reduce drinking than the alcohol only condition among heavier relative to lighter drinkers (H5b). These predictions were tested by including $\mathrm{T} 1$ drinking*treatment condition interaction terms, conditional effects, demographic variables, total interpersonal stigma, and structural stigma in the model. As shown in the top of Table 14, the interaction terms significantly contributed to the variability in alcohol use accounted for by the model, $R^{2}=.01, F \Delta(2,387)=4.24, p=.01$.

Offering partial support for 6a, the alcohol+coping PNF*baseline drinking interaction significantly differed from zero $(B=-.25, S E=.09, p=.006)$ while the alcohol only PNF*baseline drinking interaction did not $(B=-.06, S E=.08, p=.41)$.

As shown in Figure 8, when baseline alcohol use was low, both the alcohol+coping PNF condition $(B=-.34, S E=.09, p<.001)$ and alcohol only PNF condition $(B=-.46, S E=.09, p<.001)$ 
were associated with reduced drinking at follow-up, relative to control. Similarly, when baseline alcohol use was high, both the alcohol+coping PNF condition $(B=-.72, S E=.09, p<.001)$ and alcohol only PNF condition $(B=-.55, S E=.08, p<.001)$ were associated with reduced drinking. Comparing these treatment effects, the alcohol only PNF condition was slightly (but not significantly) better at reducing drinking than the alcohol+coping PNF condition when baseline drinking was low, $B=-.11, S E=.08, p=.15$. However, in support of H5b, when alcohol use was high at baseline, the alcohol+coping PNF condition significantly outperformed the alcohol only PNF condition in reducing drinking, $B=-.17, S E=.08, p=.03$.

Table 14

Summary of PROCESS models examining baseline drinking and exploratory demographic moderators of the relationship between study condition and alcohol use at follow-up $(n=400)$.

\begin{tabular}{|c|c|c|c|c|c|}
\hline Predictor & $B$ & $S E$ & $T$ & $F \Delta$ & $R^{2} \Delta$ \\
\hline Model 6: Baseline Drinking as moderator & & & & $4.23^{* *}$ & .01 \\
\hline Alcohol+Coping PNF*T1 Alcohol Use & $-.25 * *$ & .09 & $-2.72 * *$ & & \\
\hline Alcohol PNF*T1 Alcohol Use & -.06 & .08 & -.82 & & \\
\hline Model 7: Age in Years as moderator & & & & .59 & .001 \\
\hline Alcohol+Coping PNF*T1 Alcohol Use & .009 & .008 & 1.03 & & \\
\hline Alcohol PNF*T1 Alcohol Use & .002 & .007 & .32 & & \\
\hline Model 8: Relationship Status as moderator & & & & .13 & $<.001$ \\
\hline Alcohol+Coping PNF*Single & -.04 & .13 & -.31 & & \\
\hline Alcohol PNF*Single & .02 & .13 & .14 & & \\
\hline Model 9: Sexual Identity as moderator & & & & .48 & .002 \\
\hline Alcohol+Coping PNF*Bisexual & .05 & .16 & .28 & & \\
\hline Alcohol PNF*Bisexual & -.09 & .15 & -.58 & & \\
\hline Alcohol+Coping PNF*Queer & -.14 & .15 & -.89 & & \\
\hline Alcohol PNF*Queer & -.08 & .17 & -.44 & & \\
\hline Model 10: Race/Ethnicity as moderator & & & & .003 & $<.001$ \\
\hline Alcohol+Coping PNF*Racial/Ethnic Minority & -.01 & .13 & -.08 & & \\
\hline Alcohol PNF*Racial/Ethnic Minority & -.004 & .13 & -.03 & & \\
\hline
\end{tabular}

See Appendix E, Table E4 for full PROCESS model results and covariate information, ** $p<.01$ 


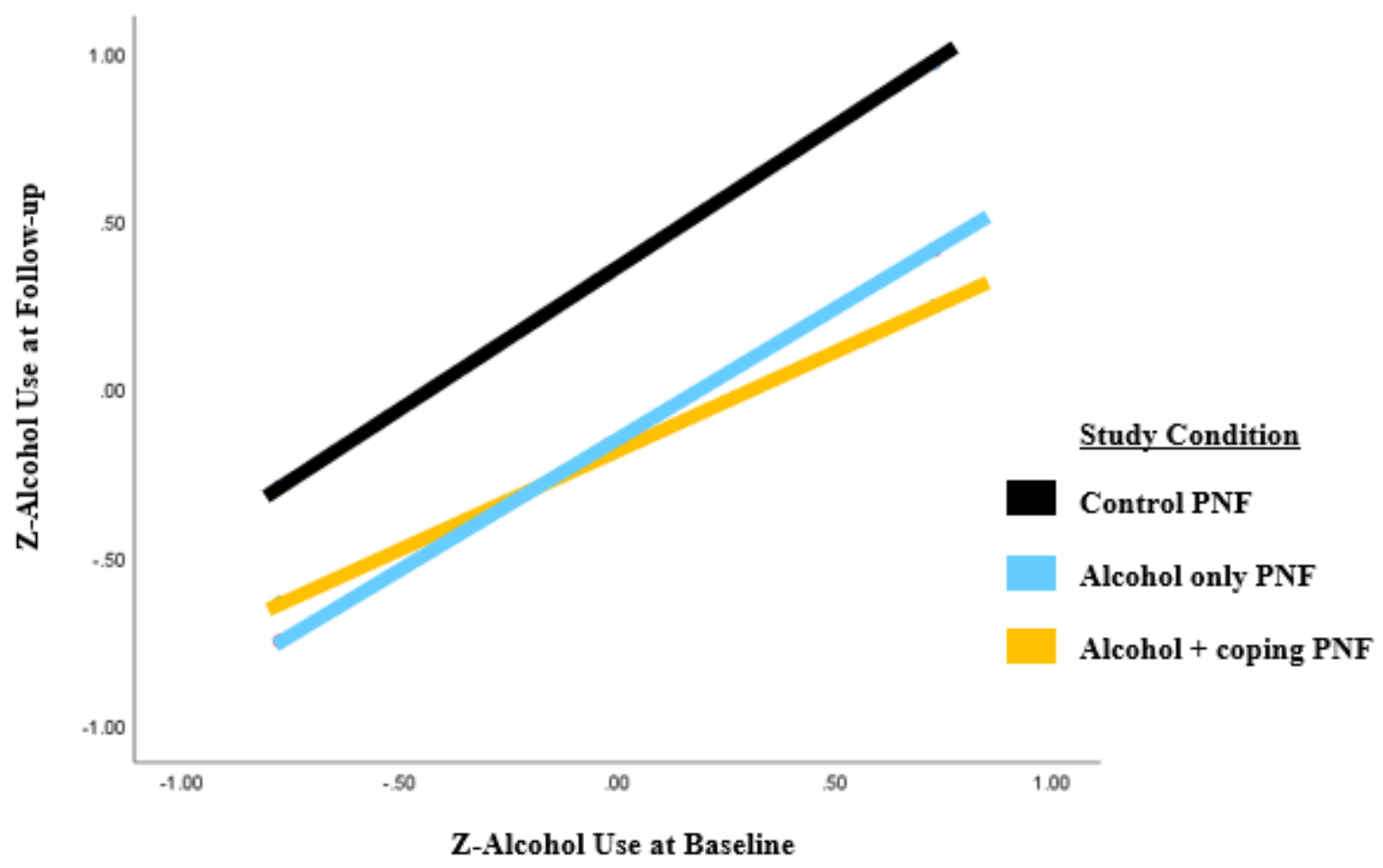

Figure 8. Alcohol use at follow-up as a function of study condition and alcohol use at baseline.

\section{Exploratory Tests of Demographic Variables as Potential Moderators}

Exploratory PROCESS models examining potential demographic moderators paralleled previous models in approach, controlling for the predictors involved in the interaction, other demographic variables, total interpersonal and structural stigma measures, and the baseline measure of alcohol use. As summarized in Table 12, none of the demographic variables moderated the relationship between condition assignment and drinking at follow-up. Thus, there was no evidence to suggest that the LezParlay treatment PNF conditions were differentially effective in reducing alcohol use as a function of participant age, sexual identity, relationship status, or race/ethnicity. 


\section{Comparison of ITT and CC Moderator Models \& Conclusions}

The only two significant moderators of relationships between PNF condition and drinking at follow-up revealed by $\mathrm{CC}$ models, interpersonal stigma exposure at $\mathrm{T} 2$ and alcohol use at T1, were re-tested in more conservative ITT models (See Appendix E). The ITT results were highly consistent with $\mathrm{CC}$ model results and probing the significant interactions in ITT models produced plots nearly identical to those from CC models (Figures 7 and 8) for CC models. The parallel moderation results obtained between ITT and CC models indicate that significant interactions supporting $\mathrm{H} 3 \mathrm{a}$ and $\mathrm{H} 5 \mathrm{~b}$ were not an artifact of participant attrition.

\subsection{Summary of Efficacy Findings}

Overall, LezParlay treatment PNF conditions meaningfully and similarly reduced alcohol use relative to PNF control topics (H1a and H1b supported), and notably, the size of LezParlay's treatment effects doubled those typically observed in the treatment arms of traditional PNF alcohol interventions in other populations of drinkers. Considered at length in Chapter 6, it is likely that characteristics of SMW as the target population and characteristics of the intervention (i.e., deep structure cultural tailoring, competition framing, theory-based game mechanics) played both individual and synergistic roles in the larger treatment effects observed in this trial.

Although main effect models did not provide any evidence that correcting norms for the coping behaviors engaged in by fellow SMW provided drinking reduction benefits beyond those associated with alcohol PNF (H2 not supported), moderator analyses did reveal one group of participants for whom additional coping focused PNF was beneficial-- those who entered the study as heavier drinkers (H6b supported). In Chapter 6, several potential explanations are considered to explain the lack of support for $\mathrm{H} 2$, and further, why the benefits associated with this condition may have been limited to heavier drinkers. 
Consistent with predictions, interpersonal stigma acutely experienced during the months between treatment and follow-up moderated the relationship between PNF condition and alcohol use such that both treatment PNF conditions were associated with larger reductions in drinking at follow-up among SMW reporting higher (relative to lower) interpersonal stigma exposure (H3a supported). However, the alcohol+coping PNF condition was not found to more effective than alcohol only PNF at higher interpersonal stigma exposure (no support for $\mathrm{H} 3 \mathrm{~b}$ ); the drinking reduction effects were quite similar between the two treatment conditions. Together these findings suggest that norms related to perceptions of LBQ peers' drinking and partying may be more salient and accessible to LBQ women following inter-group threat experiences than norms related to LBQ peers' coping behaviors or specific motivations for drinking/partying (i.e., coping with stigma).

In contrast to the support garnered for H3a, neither state nor county-level structural stigma significantly moderated these relationships (lack of support for $\mathrm{H} 4 \mathrm{a}$ and $\mathrm{b}$ ). Discussed at length in Chapter 6, these findings underscore key differences between interpersonal and structural forms of stigma and suggest that these forms of stigma may be experienced and processed differently in relation to the self, the sexual minority ingroup, and heterosexual outgroup. Finally, exploratory analyses did not identify any demographic characteristics to be significant moderators of the relationship between condition assignment and drinking at followup. Thus, there was no evidence LezParlay treatment effects were enhanced nor diminished based on participant age, sexual identity, relationship status, or racial/ethnic minority status. 


\section{CHAPTER 5}

\section{Feasibility Results}

SMW's level of interest in the LezParlay competition (i.e., total number of landing page visits, total sign-ups, and demographic characteristics of players), engagement (e.g., average numbers of logins, page views, and rounds played) as well as competition acceptability (mean ratings) and perceived benefits (i.e., the proportion reporting benefits), and alcohol use status (among all those taking part in Round 3) were examined descriptively. Qualitative text entry responses to items assessing perceived benefits of the LezParlay competition and improvements/features requested for the next version were also coded by category/theme.

\subsection{LezParlay Player Engagement}

LezParlay's informational landing page received 4,099 unique views during recruitment and competition periods. Google Analytics data indicate that promotional campaigns on HER Social App (34\%, $n=1,394)$, Facebook/Instagram (32\%, $n=1,312)$, and Google Search (23\%, $n=942$ ) were responsible for the bulk of landing page visitors. Of those visiting the landing page, $49 \%(n=2,008)$ advanced to create a user account in order to take part in the competition. In addition, 669 user accounts were created organically by users who did not first view the landing page but rather were directly invited to the LezParlay web app by a friend taking part in the competition. In total, 2,677 users signed up for the competition and most resided in the U.S. $(96 \% n=2,562)$. By metro area (see Figure 9), the largest numbers of users came from Los Angeles (11\% $n=286)$, New York City ( $8 \% n=223)$, Chicago (5\% $n=129)$, Atlanta (4\% $n=116)$, Philadelphia (3\% $n=91)$, and San Francisco/Oakland (3\% $n=86)$. Users ranged in age from 18 to 70 years, with the largest groups between 25 and 34 years $(48 \% n=1,284)$ and 35 and 44 years (21\% $n=562)$. Additional user demographics are presented in Table 16. 


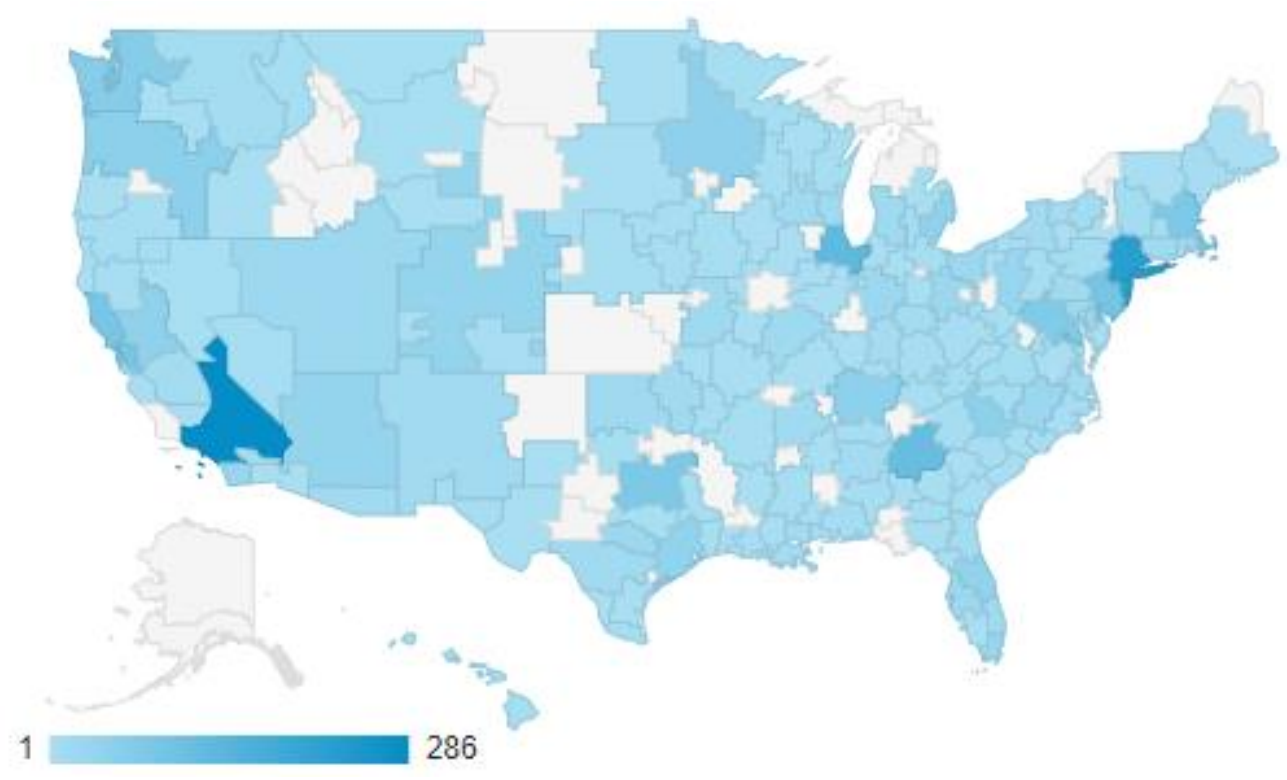

Figure 9. Geo-density of LezParlay users across U.S. metro areas

Table 16

Characteristics of LezParlay users $(N=2,667)$.

\begin{tabular}{lrc}
\hline & & $\%(n)$ \\
\hline Sexual Identity & Lesbian & $54(1446)$ \\
Bisexual & $25(669)$ \\
Queer & $21(562)$ \\
Age-group & & \\
& younger than 18 years & $4(107)$ \\
$18-24$ years & $15(401)$ \\
$25-34$ years & $48(1,284)$ \\
$35-44$ years & $21(562)$ \\
$54-65$ years & $9(240)$ \\
Relationship Status & $66+$ years & $3(80)$ \\
& Single & $45(1205)$ \\
& In a relationship & $32(857)$ \\
Married & $17(455)$ \\
Device Used & $6(161)$ \\
& It's complicated & $85(2,266)$ \\
& Mobile Phone & $2(54)$ \\
Tablet & $13(347)$ \\
\hline
\end{tabular}


Google Analytics indicated that following initial sign-up, the average player logged into the LezParlay app 3 times during the competition, completed 2.5 rounds, viewed 4.89 app pages per login, and, on average, spent 4.65 minutes on the app per login (no standard deviations available). However, it is important to note that these averages do not differentiate between players involved and not involved in the evaluation study and there were vastly different levels of retention and re-engagement efforts made based on player/study participant status. Following their initial sign-up, all new users received an automatic welcome email from the LezParlay team. Similarly, all users who completed each round received links to view their detailed results and play the next round via email and text message. However, unless users were enrolled in the evaluation study, they did not receive additional email or text message round completion reminders from LezParlay. Evaluation study participants, in contrast, received an additional email introducing each new round and 1 text message reminder 24 hours prior to the close of each round. Further, in Rounds 4 and 7, which were critical to the evaluation study, participants received up to 3 email and text-message based reminders. Further inspection of data downloaded from the backend of the LezParlay app indicated that approximately 650 LezParlay players signed up early in the competition (during rounds 1,2 , or 3 ) and remained highly engaged throughout, logging in 1 or more times per month and completing 6 or more of the 8 rounds. Of note, approximately 300 of these highly engaged players were participants in the evaluation study. Thus, although no efforts were made to retain or re-engage players not taking part in the study, and these players were not incentivized beyond the competition for a few cash prizes, LezParlay engagement remained high among approximately 350 of such players.

The LezParlay web application also recorded 44,072 total page visits among logged in users. The most visited sections of the app in terms of proportion of total page visits were browse 
profiles ( $35 \%, n=15,425$ visits) followed by play round ( $24 \%, n=10,577$ visits), view leaders \& winners (17\%, $n=7,492$ visits), submit \& vote on questions ( $13 \%, n=5,729$ visits), news/updates (9\%, $n=3,966$ visits), and edit profile ( $2 \% n=881$ visits). This web app traffic data does not include views of detailed results because the game engine files that produced these screens had to be hosted on a separate domain (www.lezparlayresults.com) to ensure that they loaded in a timely fashion. Traffic metrics provided by the domain host indicated that the detailed results site was visited a total of 7,951 times during the competition by 1,943 unique visitors. Further, visitors spent an average of 2.1 minutes viewing results each visit (no standard deviation available).

\subsection{Alcohol Use Among Players Taking Part in Round 3}

A total of 1337 players completed Round 3 where they reported on their alcohol use behaviors over the previous two months. The latest NIAAA guidelines for women define the upper-bound of "moderate drinking" to be 7 or fewer drinks per week with no more than 3 drinks consumed on any given day (NIAAA, 2017). Patterns of consumption not exceeding the moderate drinking limits are associated with low-risks for alcohol-related health problems and development of alcohol use disorder. Exceeding these guidelines is considered "heavy drinking" and associated with considerably higher health and dependence risks. Among the 1337 players who completed Round 3, 19\% ( $n=254$ ) reported no alcohol consumption or light drinking (i.e., 2 or fewer drinks per week), 26\% ( $n=346$ ) reported moderate drinking (i.e., 3-7 drinks per week, and 3 or fewer peak drinks), and 55\% ( $n=737)$ reported heavy drinking (i.e., $8+$ drinks per week or 4+ peak drinks). This sample composition was sufficient for generating risk-reducing actual norms to deliver to drinkers in the evaluation study as the lower levels of consumption among non/light and moderate drinking SMW attenuated the higher-levels of consumption among heavy 
drinking SMW. The large number of heavy drinkers who elected to take part in Round 3 before any participation incentives were offered underscore the benefits of deep structure cultural tailoring and game framing in the PNF context.

\subsection{Acceptability among Study Participants}

Overall, the 356 participants who completed the feedback survey following the competition found the LezParlay to be highly acceptable, with the average participant rating competition aspects between "liked" and "liked very much" $(M=41.26, S D=3.84$; out of a maximum score of 50). Table 17 presents descriptive statistics for acceptability items.

Table 17

LezParlay competition acceptability ratings by item $(N=356)$

\begin{tabular}{lc}
\hline Acceptability item & Rating $\boldsymbol{M ( S D )}$ \\
\hline The "stereotype challenge" concept & $4.37(.56)$ \\
The topics and questions & $4.01(.56)$ \\
Receiving the detailed results each round & $4.51(.56)$ \\
Browsing players profiles & $3.62(.66)$ \\
Submitting \& voting on questions & $3.95(.59)$ \\
Betting points on your guesses being correct & $4.34(.62)$ \\
Receiving text message reminders & $4.12(.58)$ \\
Receiving email reminders & $4.27(.50)$ \\
Viewing the top scorer leaderboards & $3.89(.60)$ \\
Competing for money \& receiving gift cards & $4.09(.55)$ \\
\hline
\end{tabular}

Note. Response options ranged from (1) disliked very much to (5) liked very much.

Exploratory analyses examined correlations between acceptability and participant-level characteristics. Acceptability ratings were positively correlated with racial/ethnic minority status $(r=.32, p<.001)$, county-level structural stigma $(r=.22, p<.001)$, and state-level structural stigma ( $r=.17, p=.001$ ), with a greater liking of LezParlay observed among racial/ethnic minorities and those living in communities marked by greater structural stigma. Acceptability 
ratings were not significantly related to participant age, sexual identity, baseline alcohol use, relationship status, nor either measure of interpersonal stigma.

The highest rated aspect was receiving the detailed results each round $(M=4.51 S D=.56)$ and ratings did not differ by study condition, $F(2,355)=.41, p=.67$, suggesting that participants liked the feedback on treatment topics just as much as control participants liked feedback on the non-health-related topics. The lowest rating among the 10 items, browsing player profiles $(M=3.62 S D=.66)$, was surprising in the context of traffic data which revealed the browse profiles section of the app to be the most frequently visited section. This low rating may be explained by participants desiring greater between-player interactivity and is discussed later in the context of responses to questions about LezParlay perceived benefits and feature requests.

\subsection{Perceived Benefits among Study Participants}

Overall, 93\% ( $n=331)$ of participants completing the feedback survey found the LezParlay competition to be psychologically beneficial and $85 \%(n=283)$ of these participants described perceived benefits through the optional free-response item. Coding sought to condense the raw text descriptions submitted by participants into a summary of common benefit categories. The generic inductive qualitative coding approach described by Thomas (2006) was used to accomplish this. Similar to grounded theory, but more appropriate when the coding objective is summarizing phenomena for basic understanding rather than building theory (Liu, 2016), this iterative approach is notably subjective and prone to reflecting the perceptions and biases of the coder. As such, diverse perspectives were sought for the benefit coding task with the lesbian-identified senior researcher, a gay male-identified senior researcher, and two heterosexual female research assistants taking part in this process. First, the two senior researchers conducted independent, initial readings of benefit descriptions, with each aiming to 
identify no more than 10 unique categories of benefits. As responses were generally short but many described more than 1 benefit, it was decided a priori that each response could receive up to 3 category classifications. The senior researchers then met, compared and contrasted categories, agreed upon common category themes, and identified several pairs of categories that were extremely similar and could be condensed into a single category. This process culminated in 6 shared benefit categories. Next, two research assistants independently classified all 283 responses according to the 6 categories, with each response coded for a maximum of 3 benefits. Inter-rater reliability was high $(\mathrm{Kappa}=.91)$ and discrepancies were resolved through discussion among senior researchers and research assistants. Table 18 presents the proportion of total responses that reflected each benefit category and representative responses of benefits in each category. 
Table 18

Categories of psychological benefits described by participants and representative responses

\begin{tabular}{|c|c|c|}
\hline Benefit Category & $\begin{array}{l}\% \text { of Total } \\
\text { Responses }\end{array}$ & Representative Responses \& Responder Characteristics (sexual identity, age) \\
\hline $\begin{array}{l}\text { Knowledge/ } \\
\text { Social Comparison }\end{array}$ & $65 \%$ & $\begin{array}{l}\text { I liked getting to see the results and learn about the community since I live in a rural area and am pretty isolated. - } \\
\text { Bisexual, } 28 \text { years old } \\
\text { The questions helped me evaluate my experience as a young lesbian, and the results gave me insight about how my } \\
\text { community is doing and how my experiences compare. -Lesbian, } 21 \text { years old } \\
\text { I work at an LGBTQ community center and it really helped having data to influence our programs and identify } \\
\text { topics/issues to discuss in our women's group meetings. -Queer, } 41 \text { years old } \\
\text { Let me learn more about the lgbtq community and see that I drink way more than average lol fail } \\
\text {-Lesbian, } 38 \text { years old }\end{array}$ \\
\hline $\begin{array}{l}\text { Community Connection/ } \\
\text { Identity Strength }\end{array}$ & $34 \%$ & $\begin{array}{l}\text { It helped me feel more understood and like I really wasn't the only one that felt this way about things or the only one to } \\
\text { have dealt with something. In a weird way I kind of felt more connected to the community as a whole just by being able } \\
\text { to see the way others responded too. -Bisexual, } 30 \text { years old } \\
\text { I don't really go out to queer bars and clubs much so this was a cool way to feel connected to the community from the } \\
\text { comfort of my home! Also helped me not feel so alone in my experiences. -Lesbian, } 27 \text { years old } \\
\text { Being in my 50s and feeling sort of invisible these days this competition really helped me feel connected to something } \\
\text { again -Lesbian, } 52 \text { years old } \\
\text { It was so great for me although it's hard to describe how/why exactly...felt connected and in the know.... also felt more } \\
\text { confident and secure in my identity. -Queer, } 25 \text { years old }\end{array}$ \\
\hline Stigma/Bias Reduction & $31 \%$ & $\begin{array}{l}\text { This really helped me reduce biases that I had internalized without even realizing it! -Queer, } 26 \text { years old } \\
\text {...honestly feel like this competition helped reduce biases I had about other LGBTQ groups too... like no in fact bisexual } \\
\text { women DON'T always cheat and act shady in relationships! -Lesbian, } 40 \text { years old } \\
\text { Cool to see that some of the negative ways we get portrayed in the media are totally off. -Lesbian, } 36 \text { years old } \\
\text { I liked to see how the stereotypes werent necessarily right. It felt refreshing to know that we dont fit in to one singular } \\
\text { box. -Lesbian, } 29 \text { years old } \\
\text {...helped me understand the shared experiences of bisexuals and made me feel much better that some of the negative } \\
\text { stereotypes were shattered! Also corrected some not-so-great assumptions I had about lesbians. -Bisexual, } 34 \text { years old }\end{array}$ \\
\hline
\end{tabular}




\begin{tabular}{|c|c|c|}
\hline $\begin{array}{l}\text { Introspection/ } \\
\text { Self-confrontation }\end{array}$ & $23 \%$ & $\begin{array}{l}\text { This really helped me see that I need to get my shit together in several areas -Lesbian, } 33 \text { years old } \\
\text { Made me question some of my own tendencies and behaviors. Came to see that I was doing what I thought everyone else } \\
\text { was doing which wasn't even the truth.... -Lesbian, } 28 \text { years old } \\
\text { It made me really question the stereotypes I'd had. Like, with uhauling, I kinda realized that it was one of those things } \\
\text { that is more uncommon than I thought. I kinda now understand it to be something where everyone I know personally } \\
\text { knows someone who did it (lol, I was that one in my group), but most of us haven't actually done it ourselves. -Bisexual, } \\
30 \text { years old } \\
\text { It was especially helpful for discerning what stereotypes I have internalized and which aspects of my life are "normal" } \\
\text { within the context of fellow queer women and overall LBQT women. I felt heard, visible, understood, affirmed, and } \\
\text { gently held accountable. -Queer, } 43 \text { years old }\end{array}$ \\
\hline Fun/Entertainment & $19 \%$ & $\begin{array}{l}\text { Loved the competition, betting, prizes, and leaderboards... so so fun -Lesbian, } 51 \text { years old } \\
\text { I felt like I learned a lot and I'm a competitive person, so the competition just brought more fun to it and made me look } \\
\text { forward to playing each round. -Queer, } 24 \text { years old } \\
\text { It was so fun and I was able to get my lesbian roommate to play with me... we got super competitive about scores and } \\
\text { had a blast! -Bisexual, } 37 \text { years old } \\
\text {...It was fun to feel like our community has a space like this as well. I've never played a game that involved my sexual } \\
\text { identity or orientation. You all are rad! -Queer, } 29 \text { years old }\end{array}$ \\
\hline $\begin{array}{l}\text { Mood Enhancement/ } \\
\text { Positive Outlook }\end{array}$ & $14 \%$ & $\begin{array}{l}\text {...helped my mental health and gave me a more positive outlook on all things queer. - Queer, } 29 \text { years old } \\
\text { It just felt really good... like someone actually wanted to know more about my opinions/experiences } \\
\text {-Bisexual, } 27 \text { years old } \\
\text { It was a source of enjoyment. Getting the results always put me in such a good mood... even when I was wrong about } \\
\text { stuff... -Lesbian, } 23 \text { years old } \\
\text { Although a lot of negative stereotypes are not true, we do have some shared experiences and highlighting these } \\
\text { positive/uniquelfunny things really put me in a good mood -Lesbian, } 33 \text { years old }\end{array}$ \\
\hline
\end{tabular}


The largest proportion of responses $(65 \%)$ reflected benefits in the domains of learning and social comparison, with many participants commenting on the value they found in the real, community derived statistics and rare opportunity to directly evaluate their experiences and behaviors. Several participants describing these types of benefits as well as those related to stigma reduction and introspection/self-confrontation expressed that LezParlay's results made them ponder the origins of stereotypes and reinforcing factors. Numerous SMW identified problematic media representations and portrayals of LBQ women in television shows and movies to be at the root of stereotypes they had internalized about poor physical health among LBQ women (e.g., I really thought queer/les women smoked and drank way more because we always are on tv shows and movies... It was great to challenge those notions, even if it meant my score suffered). Other participants also reported that they were surprised and encouraged by the statistics revealing less health-risk behavior (i.e., heavy drinking, smoking) or more preventative health behavior (exercise, wellness check-ups) than they expected among peers. Among participants presumably not engaging in risk-behavior themselves, this health-related reality check seemed to improve their outlook (i.e., you constantly hear how LGBTQ people are at medical disadvantage statistically, which can be really disheartening... So seeing how much people are out there also trying to take care of themselves was great). Meanwhile, the same statistics presumably led unhealthy participants to realize that they were in the minority, which, in turn, appeared to motivate change (e.g., this really helped me see that I really need to get my shit together in several areas).

Many of the psychological benefits reported extended beyond those which may have been experienced in a traditional PNF alcohol intervention, mapping directly onto individual game mechanics, the constellation of gamified elements, or the broad challenging of negative 
stereotypes. For example, potentially mapping onto SDT's satisfaction of autonomy and competence needs, $19 \%$ of responses indicated that participants found the competition fun and entertaining, and $14 \%$ stated that taking part in LezParlay enhanced users' mood or improved their outlook. Further, 33\% of the benefits described suggested satisfaction of relatedness needs, revealing that LezParlay helped users feel less alone in their experiences, more connected to the LBQ community, or stronger in their identity. Such relatedness benefits seem attributable to both the virtual community of LBQ women made possible by LezParlay's social media-inspired copresence features and other aspects of the competition that reflected deep structure cultural tailoring (e.g., receipt of detailed results across a multitude of salient issues and shared experiences).

The stigma reduction benefits reported by participants were also unexpectedly farreaching. Whereas the larger competition was intended to challenge negative stereotypes about one's own sexual identity group and many participants' responses reflected benefits in this domain, some participants reported de-stigmatization benefits that stretched beyond their own identity group. That is, although only a single results screen for each topic compared users' experiences and behaviors to users in other sexual identity and age groups (most results screens were matched to the user's own sexual identity and age-group), a handful of lesbians commented that seeing the statistics (i.e., actual norms) for bisexual women improved their perceptions of bisexuals (i.e., honestly feel like this competition helped reduce biases I had about other LGBTQ groups too... like no in fact bisexual women DON'T always cheat and act shady in relationships), a few bisexual women reported that results helped reduce their negative perceptions of lesbians, while others increased their understanding of SMW who identify as "queer", an identity term still misunderstood in many parts of the U.S. Similarly, a handful of 
older participants reported that they appreciated the opportunity to learn about the experiences of younger LBQ women, given the drastic changes in social climate for sexual minorities over the past 20-30 years.

\subsection{Participants' Ideas \& Requests for the Next Version}

A subset of participants submitted ideas and/or feature requests for the next version of LezParlay ( $n=169)$, while other participants used this text entry box to express that they were pleased with the competition in its initial form $(n=43)$. The same two-step coding procedure was used to identify themes across requests/ ideas and inform the next version of the competition. As many responses contained multiple requests or ideas, a total of 307 total items were coded. Interrater agreement was near perfect (Kappa=.98). Among requests and ideas suggested by multiple participants, participants most commonly desired a native (IOS/Android) smartphone app (36\%, $n=112)$, more frequent rounds with faster results delivery $(24 \%, n=74)$, increased opportunity for interaction between players (e.g., a chat feature or direct messaging that could be turned on/off; $14 \%, n=43$ ), increased ease of inviting friends and/or the ability to earn bonus points for referring friends $(8 \%, n=24)$, the ability to go back and change previously submitted guesses or point wagers prior to the round closing $(4 \%, n=12)$, the connection of results to informational articles or community resources $(4 \%, n=11)$, additional questions about race, gender identity, and/or sexual identity-based biases within the community $(3 \%, n=9)$, world-wide promotion and additional results comparing the behaviors/experiences of LBQ players in different countries/geographic regions $(1 \%, n=4)$, the ability to see the profile photos of the group members being guessing about on the guess question screens $(1 \%, n=4)$, the addition of a youth/teen age-group to help those just coming out $(1 \% n=4)$, live, "game-show" like rounds at community events (in addition to the on-going app-based competition, $1 \% n=3$ ), no betting of 
points at all (instead correct answers worth a flat amount of points, 1\%,n=3) and the ability to earn bonus points for tagging heterosexual friends on LezParlay Instagram posts documenting shattered stereotypes outside of the app $(1 \%, n=2)$.

It is notable that the third most common request among participants was increased opportunities for interaction between users. The desire for direct messaging or chat functionality among users explains the disconnect observed previously between the high levels of engagement with the browse profiles section of the app and community connection benefits widely reported, yet lower acceptability rating of the ability to browse profiles relative to other aspects of the competition. In sum, responses suggest that the virtual community aspect of LezParlay was highly valued and beneficial; however, participants desired greater opportunities for connection through interaction than the initial version of LezParlay afforded.

\subsection{Summary of Feasibility Findings}

LezParlay's large number of sign-ups (i.e., 2,677 user accounts), the high level of userengagement (i.e., 44,072 web app page views), the large number of heavy drinkers engaged by the competition, the risk-reducing drinking norms organically generated among users, and positive feedback among evaluation study participants support for the feasibility and scalability of this gamified alcohol intervention approach. From a health intervention standpoint, it is promising that there were no requests for less health-related feedback or a greater proportion of feedback on fun versus serious topics. Further, receiving the detailed results (i.e., PNF) was the most liked aspect of LezParlay among drinkers taking part in the evaluation study, with ratings not significantly associated with study condition or level of alcohol consumption. This suggests that those receiving less and more health-related results as a function of condition and those entering the study as lighter and heavier drinkers similarly enjoyed receiving the PNF delivered. 
These findings suggest that future versions of the competition might also correct additional types of alcohol and coping-related norms or expand the topics on which PNF is delivered to other areas of physical and mental health, without detracting from acceptability or engagement.

Participants submitted a variety of thoughtful ideas and actionable requests for the next version of LezParlay, which illuminated their overall enthusiasm for the competition and desire for continued participation. Most participants (93\%) also reported that they psychologically benefitted from taking part in competition and descriptions of benefits reflected learning and social comparison, community connection and identity strength, stigma reduction, enhanced mood/outlook, and entertainment. Many of these benefits mapped onto LezParlay's constellation of game mechanics and deep structure cultural tailoring efforts, and importantly, stretched beyond those that would have benefitted participants taking part in a traditional alcohol focused PNF intervention. In particular, participant responses revealed unanticipated but promising psychological benefits related to both the larger competition's social media-inspired copresence features and delivery of non-treatment PNF that challenged negative stereotypes related to identity experiences, health, relationships, and sexual behavior. Discussed further in Chapter 6, the next version of LezParlay will draw on these insights as well as feature requests submitted by participants in efforts to maximize psychological benefits, competition acceptability, and alcohol intervention efficacy. 


\section{CHAPTER 6}

\section{Discussion}

SMW remain among the heaviest drinking female populations; however, culturally tailored, evidence-based alcohol interventions for this population have been slow to emerge (Blume, 2016; Hughes et al., 2020). As research suggests that the centrality of alcohol use in queer socialization contexts encourage positive associations between alcohol use and sexual minority identity (e.g., Parks, 1999; Parks \& Heller, 2013; Parks, Hughes, \& Kinnison, 2007) and misperceptions of heavy drinking as normative (e.g., Boyle et al., 2020; Ehlke et al., 2019), there is an unmet need for theory-driven interventions able to attract, engage, and motivate behavior change among heavy drinking SMW who do not yet view their consumption as excessive. Informed by both minority stress and social norms based explanations for heavy drinking among SMW, the LezParlay intervention leveraged digital game mechanics informed by SDT (Ryan \& Deci, 2000; Ryan et al., 2006) and deep structure cultural tailoring to deliver PNF designed to correct misperceived peer norms for drinking and stigma-coping behaviors. This competition-based, incognito intervention was intended to both remedy the attentional and motivational limitations associated with traditional web-based PNF interventions and increase attraction and engagement among LBQ drinkers. A hybrid trial design allowed key questions related to the LezParlay intervention's feasibility and efficacy to be cost-effectively evaluated within the same study. The sections that follow provide discussions of findings addressing this study's major feasibility and efficacy questions and implications for future research.

\subsection{Overview of Findings}

\section{Did the gamified intervention packaging engage SMW?}


As the literature has documented much difficulty in engaging and retaining drinkers in traditional web-based PNF alcohol interventions when participation is not mandatory and monetary incentives are not offered at the point of study recruitment (e.g., Dempsey et al., 2018; Palfai et al., 2014), a major feasibility question was whether LezParlay’s packaging as a culturally tailored social media-inspired competition could attract and engage SMW—and, in particular, heavy drinkers, when traditional study incentives were not advertised or offered at the point of sign-up. Supporting the feasibility of this approach and far exceeding recruitment goals, 2,677 SMW diverse in age, race/ethnicity, alcohol consumption level, and geographic location signed up to take part in the competition. Further, analysis of alcohol use data organically collected among users taking part in the competition's third monthly indicated that more than half of the users met NIAAA criteria for high-risk drinking and thus, were an ideal population for PNF intervention. Importantly, the markedly lower levels of consumption among alcohol abstainers and low-risk drinkers also taking part in the round attenuated the levels of consumption among heavy drinkers, allowing risk-reducing actual drinking norms (presented in PNF) to be organically generated in real-time from users completing the round.

Despite the large number of high-risk drinkers taking part in LezParlay, the actual quantity and frequency drinking norms generated among lesbian and bisexual players in their 20s and 30s were roughly equivalent or in some cases lower than the corresponding actual drinking norms derived from recent alcohol focused survey samples of lesbian (Boyle et al., 2020) and bisexual women (Ehlke et al. 2019) in these age groups. As previous survey study samples were comparatively small and did not include queer-identified women or LB women in older age groups, a traditional PNF intervention approach for this population would have required that a large, diverse sample of LBQ women first take part in a separate norms documentation survey 
study prior to recruiting another large LBQ sample to take part in the intervention. For hard-toreach populations, this norms documentation step can be particularly time-consuming and costly (e.g., advertisements, participant incentives). Thus, LezParlay's ability to attract and engage SMW diverse in alcohol experience so that risk-reducing norms could be featured in PNF is a major point of innovation that drastically decreased research costs by eliminating the need for a separate norms documentation study. Further, as suggested by previous findings with college students (Boyle, Earle, et al., 2017; LaBrie et al., 2019), presenting actual norms transparently derived from the real-time responses of visible peers may have made treatment PNF more impactful than it would have been had previously collected data from a different, faceless sample of SMW been presented. Supporting this idea, the social media-inspired copresence features appeared to be integral to SMW's interest and engagement in LezParlay as evidenced by the browse player profiles section of the app receiving more traffic than any other, and many participants' describing benefits associated with the visible virtual community aspect of the competition.

\section{Did correcting sexual identity and age-specific drinking norms reduce SMW's alcohol use?}

Immediately following LezParlay’s third round, a sub-sample of 500 moderate to highrisk drinkers agreed to help evaluate LezParlay by participating in an evaluation study where they were randomized to receive PNF on treatment (i.e., alcohol only, or alcohol+control) or control topics over two consecutive rounds. Consistent with previous findings (e.g., Boyle et al., 2020; Ehlke et al., 2019), at baseline the frequency and quantity with which these drinkers perceived age and sexual identity specific peers to consume alcohol exceeded the actual age and sexual identity specific drinking norms transparently derived from SMW taking part in the larger competition. Importantly, analysis of follow-up data collected two months after the delivery of 
treatment/control PNF indicated that both treatment conditions similarly and substantially reduced drinking relative to control (H1 supported, $d=.49-.50$ using the most conservative ITT estimates).

In contrast to the moderate effect sizes associated with LezParlay's treatment conditions, the treatment arms of traditional web-based PNF alcohol interventions remotely delivered to university and military populations of drinkers are typically associated with smaller effects ( $d=.18$-.26) across drinking outcomes 1 to 2 months post-intervention (e.g., Dotson et al., 2015, Pederson et al., 2016, Walters \& Neighbors, 2005). As problems with motivation (e.g., Bedendo et al., 2019a), inattention (e.g., Rodriguez et al., 2015), and defensiveness (e.g., Steers et al., 2016) have been theorized to undermine the efficacy of web-based PNF interventions in other populations of drinkers, LezParlay's competition framing and game mechanics sought to mitigate these negative forces and likely contributed, in kind, to the enhanced treatment effects observed. Suggesting the benefits of these efforts, LezParlay's treatment effects are most consistent with those observed by Earle and colleagues (2018) in treatment arms of CampusGandr, a multi-round gamified PNF alcohol intervention for first-year college students ( $d=.33-.46 ; 2$-month follow-up). Although the most effective CampusGandr treatment condition corrected an additional type of norm not addressed in LezParlay, this intervention included several overlapping SDT-informed game mechanics (i.e., user-generated questions, chance-based uncertainty, a system of points, and player avatars) but lacked other features (i.e., no point-based wagers, round-specific leaderboards, or browsable social media-inspired player profiles).

Comparing treatment effect sizes while accounting for similarities and differences between the two interventions suggests that the efficacy associated with LezParlay's treatment conditions 
may further be increased by expanding the types of alcohol-related norms corrected. This idea is explored further later in this chapter as a priority direction for future research.

As this was the first study to examine the efficacy of a PNF alcohol intervention of any format among SMW, characteristics of this population may have also contributed to the larger effect sizes observed. For example, previous research has proposed that sexual minorities may be more interested than other populations of adults in how their behaviors and attitudes compare to peers', potentially making health intervention strategies focused on norms particularly impactful (Kuerbis et al., 2014). Traditional alcohol focused PNF interventions for other populations have also exhibited increased efficacy among subgroups of drinkers who share characteristics with the SMW in the current trial, including females (e.g., Murphy et al., 2004), those reporting drinking to cope with negative affect (e.g., Young et al., 2016; Young et al., 2018), and those for whom reference groups have higher self-relevance (Neighbors et al., 2010). Meanwhile, the LezParlay intervention was intentionally crafted to appeal to SMW strongly identified with LBQ communities, ensuring that sexual identity-specific norms corrected in the competition would be self-relevant. It seems most likely that the characteristics of this particular population worked synergistically with the intervention's deep structure cultural tailoring and game mechanics to bolster alcohol PNF efficacy.

Together, support for $\mathrm{H} 1$ and the null findings for exploratory tests of demographic moderators suggest the adequacy of LezParlay's age and sexual identity-specific reference groups. Alcohol PNF specific to these reference groups was effective in reducing participant's drinking overall and moderator analyses revealed no evidence that treatment PNF was differentially effective as a function of age, race, ethnicity, sexual identity, or relationship status. Considered together with preliminary demographic findings, this suggests that although these 
demographic characteristics may be cross-sectionally associated with levels of alcohol consumption and perceptions of peer drinking norms (e.g., single participants consumed more alcohol and perceived LBQ peers to drink more than did coupled participants), these demographic factors do not play a meaningful role in the degree to which perceived LBQ drinking norms predict alcohol use. Thus, these findings are largely consistent with broader alcohol prevention and intervention literature's consideration of PNF as a gold standard, universal brief intervention strategy effective in reducing alcohol-related risks among drinkers irrespective of their demographic characteristics (e.g., Cronce et al., 2018; Dotson et al., 2015).

Viewing support for $\mathrm{H} 1$ in the context of the larger LGBTQ health literature, it is important to highlight the fact that the statistical models yielding treatment effects controlled for measures of interpersonal and structural sexual minority stigma exposure. That is, although exposure to these and other forms of sexual minority stigma are considered the most focal antecedents to heavy drinking and alcohol dependence among SMW (for reviews see, Green \& Feinstein, 2012; Hughes et al., 2020; Talley, 2012), findings from this study underscore the utility of sexual identity-specific peer drinking norms as additional intervention targets and the appropriateness of alcohol PNF as an intervention strategy for SMW and potentially other sexual minority groups.

\section{Did correcting norms for peers' coping behaviors further reduce SMWs' alcohol use?}

Although similar percentages of drinkers in the evaluation study over-estimated norms for peer's alcohol consumption (74\%) and misperceived norms for peers' coping behaviors (69\%), there was not a significant main effect indicating that coping PNF provided benefits beyond the delivery of alcohol PNF alone (H2 not supported), nor was alcohol+coping PNF found to be more risk-reducing than alcohol PNF alone in the context of greater interpersonal or 
structural stigma exposure (no support for $\mathrm{H} 3 \mathrm{~b}$ or $\mathrm{H} 4 \mathrm{~b}$ ). Yet, moderator analyses revealed one group of participants for whom the addition of coping PNF was incrementally beneficial - those who entered the study as heavier drinkers (H5b supported). There are several potential explanations as to why the benefits associated with the coping PNF were limited to heavier drinkers. One possibility is that those entering the study as heavier drinkers used alcohol to cope with stress and stigma substantially more than did those who entered the study as lighter drinkers. This explanation is generally consistent with the sexual minority stress perspective on alcohol consumption among SMW, which explains heavier drinking in this population largely in terms of maladaptive stress coping (e.g., Dworkin et al., 2018; Lewis et al., 2016). Thus, correcting misperceived coping norms in addition to general drinking norms may have helped heavier drinkers reduce their tendency to use alcohol when faced with stress and stigma, which in turn, decreased their overall consumption. Lighter drinkers, in contrast, may not have been engaging in maladaptive coping behaviors to begin with; thus, for these participants, the coping PNF did not confer additional benefits.

Another potential explanation lies in coping behaviors having been intervention targets in the current study but not assessed as outcomes. The design of this study assumed that reductions in coping-motivated drinking would be reflected in global alcohol use outcomes (i.e., quantity and frequency of consumption, negative alcohol-related consequences). However, it is possible that maladaptive coping may contribute to global alcohol use patterns to a lesser degree than assumed among light and moderate drinking LBQ women. If true, this means that coping strategies may have shifted toward more healthful and adaptive behaviors following delivery of coping PNF among these participants; however, these changes were simply not picked up by the global drinking outcomes. 
A very different but entirely plausible explanation for these results is that coping PNF did not impact alcohol use among heavy drinking SMW by means of changing their coping behaviors. This possibility is consistent with previous research with college students and military veterans, which has found alcohol only PNF to have increased efficacy among participants who more frequently drink to cope with negative affect (Young et al., 2016; Young et al., 2018). Whereas the current study assumed that drinking to cope with general negative affect in these populations is qualitatively different or less common than drinking to cope with sexual minority stigma among SMW, this assumption may have been faulty. Instead, drinking to cope with general negative affect and sexual minority stigma may similarly enhance the efficacy of alcohol only PNF. Thus, because coping PNF corrected norms for coping-motivated drinking (among other behaviors) and was delivered one month following the initial treatment PNF on alcohol use in the current trial, it may have acted as a "booster" to alcohol PNF, further reinforcing the idea that peers of the same sexual identity and age do not drink as much as one previously thought. This potential explanation is also supported by the reciprocal nature of relationships between perceived drinking norms and drinking behavior observed in longitudinal research with SMW (Litt et al., 2015) and research into social network effects on alcohol intervention outcomes (Reid, Carey, Merrill, \& Carey, 2015). These findings suggest that with passing time, heavy drinkers' corrected drinking norms might be more likely to be re-inflated by observations of heavy drinking peers than would be the case among lighter drinkers following a single dose of alcohol PNF. Thus, it's reasonable that heavier drinking SMW might substantially benefit from such a "PNF booster," whereas the same may not be true among SMW entering the study as lighter drinkers. 
Unfortunately, the current trial design, wherein coping norms and behaviors were assessed for the first and only time one month after the baseline assessment of drinking and shortly after the delivery of alcohol PNF prohibits direct testing of potential explanations. Additional studies with multiple assessments of coping-related norms and behaviors are needed to better understand the mechanisms by which coping PNF benefitted only heavier drinking SMW in this trial. Although the null main effect for coping PNF was somewhat underwhelming, there were no subgroups of participants for whom the addition of coping PNF was found to be significantly less risk-reducing than alcohol PNF only. Thus, given its enhanced drinking reduction effects among heavy drinking SMW and the low costs associated with delivering PNF on additional topics in the current multi-round gamified intervention format, future PNF intervention studies with this population should seek to retain and further build on coping PNF to bolster and extend the benefits associated with this component.

\section{Did sexual minority stigma exposure enhance or diminish the efficacy of treatment PNF?}

Consistent with previous cross-sectional findings (e.g., Lehavot et al., 2011; Lewis et al., 2016; Nawyn et al., 2000; Slater et al., 2017), greater exposure to interpersonal stigma (i.e., sexual identity-based violence and harassment) was cross-sectionally associated with both alcohol consumption and negative-alcohol-related consequences at baseline. Meanwhile, state and county-level measures of structural stigma were significantly associated with negative alcohol-related consequences but not the frequency or quantity of alcohol consumption. Preliminary analyses also revealed that interpersonal and structural stigma exposure were positively associated with perceptions of norms for both alcohol consumption and negative alcohol-related consequences, suggesting that stigma and social norms based explanations for 
increased alcohol consumption among SMW may be less separate and more related than currently positioned in the literature.

When these forms of sexual minority stigma were examined as potential moderators of intervention efficacy, findings diverged considerably. Consistent with $\mathrm{H} 3 \mathrm{a}$, interpersonal stigma experienced in the months following PNF delivery moderated treatment PNF efficacy such that exposure to greater interpersonal stigma enhanced the drinking reduction effects associated with treatment PNF. Preliminary analyses examining cross-sectional relationships between interpersonal stigma exposure, perceived drinking norms, and alcohol use at baseline aid in the interpretation of the significant interaction at follow-up. At baseline, consistent with previous findings among gay men (Hamilton \& Mahalik, 2009), greater interpersonal stigma exposure strengthened the relationship between perceived sexual identity specific drinking norms and alcohol use among SMW. This finding suggests at follow-up in both treatment and control conditions, greater interpersonal stigma exposure (T2) similarly strengthened drinking normsbehavior relationships. However, as over-estimated peer drinking norms were only highlighted and corrected in treatment conditions, the increased motivation to conform to group norms resulted in enhanced reductions in drinking among treatment participants at follow-up relative to control. Notably, the interpersonal stigma items in this study assessed rather severe experiences with sexual identity-based violence and harassment instigated by members of the heterosexual outgroup. Salient, disruptive, and distressing, these experiences are highly consistent with the types of threats to self that theories of group dynamics (i.e., SCT, TMT) suggest would make a person likely to turn to salient in-groups for behavioral guidance.

Broadly, these findings suggest that experiencing sexual identity-based violence and harassment can make sexual minority individuals more motivated to conform to salient and 
accessible LGBTQ community norms and more susceptible to normative influence from LGBTQ peers. However, health interventions and programs designed to reduce substance use among sexual minorities adolescents and adults tend to be narrowly focused on affirming sexual minority identities, aiding individuals in understanding sexual minority stress processes, and providing resources to help individuals more adaptively cope with stigmatizing experiences (Chaudoir et al., 2015). Very few, if any, programs evaluated or in development also include PNF or other social norms intervention components. Findings from the current study provide additional direct support for the notion that the efficacy of LGBTQ-specific health interventions and treatment programs might be substantially increased through the addition of norm-focused intervention components such as PNF (e.g., Boyle et al., 2017).

In contrast, neither state, county, nor combined measures of structural stigma moderated relationships between perceived norms and drinking behavior at baseline or enhanced/diminished the efficacy of treatment PNF at follow-up (lack of support for H4a). As structural stigma is operationalized through secondary data sources, little is known about how it is psychologically experienced or processed at the individual level. However, this study's findings suggest that structural stigma may not be perceived nor experienced by SMW in ways that make sexual minority social identity feel threatened, as required for the self-stereotyping ingroup conformity effects specified by SCT, nor mortality salient, as required for the worldview-related cultural conformity effects dictated by TMT. Rather, sexual minority structural stigma may be more akin to a dull sense of social disadvantage or that quietly operates in the backdrop of daily life and leads to a general mistrust in heterosexist institutions and power structures. Support for outgroup mistrust as an experiential aspect of structural stigma comes from previous findings demonstrating the detrimental effects that race-based structural stigma can have on psychosocial 
interventions. Specifically, findings from a meta-analysis of HIV prevention interventions found that programs improved condom use among African Americans only when participants' home communities had relatively positive attitudes toward African Americans and lower levels of segregation (i.e., lower race-based structural stigma; Reid et al., 2014). However, the negative impact of race-based structural stigma was lessened in interventions that tailored content to participants' social identities and included affirmational racial pride components. Reid and colleagues theorized that these components worked to reduce African American's stigma-based mistrust in intervention programs and research teams. In contrast, in the current study, greater LezParlay competition acceptability was associated with both living in a community high in sexual minority structural stigma and being a racial or ethnic minority. Taken together with findings by Reid et al. (2014), this finding suggests that LezParlay's deep structure cultural tailoring, game framing as a competition designed to challenge negative stereotypes about the target population, creation by an ingroup member, and joint promotion by trusted ingroup community organizations may have circumvented feelings of mistrust that may have otherwise undermined traditional alcohol intervention efforts among SMW residing in high structural stigma locales. As the literature suggests that most health intervention and prevention efforts for sexual minorities have been limited to surface-level cultural tailoring (e.g., Bauer \& Wayne, 2005; Talley, 2013), the current findings underscore the utility of also incorporating deep structure adaptations.

The diverging findings for interpersonal and structural forms of stigma as moderators of PNF intervention efficacy in the current study also point to other important directions for future research. For instance, as the interpersonal stigma experiences assessed in this study were relativity severe (violence, harassment), future research should also examine whether recent and 
historical exposure to interpersonal stigma at different levels of severity (i.e., family rejection, childhood bullying, microaggressions) moderate relationships between perceived substance use norms and substance use behaviors among sexual minorities. In addition, whereas the current study productively drew upon self-categorization and terror management theories to make predictions about PNF efficacy in the context of stigma, other research and theory may also provide valuable insights into these relationships as well as aid in the identification of individual difference variables that might further modulate norm and stigma relations. For instance, rejection sensitivity (RS) is an individual difference variable that has been dually linked to increased ingroup conformity in the face of outgroup rejection by social psychologists (e.g., Knapton, Back, \& Back, 2017; Romero-Canyas, et al., 2010) and increased substance use among sexual men residing in high structural stigma locales in the LGBTQ health literature (e.g., Pachankis, Hatzenbuehler, \& Starks, 2014). Although yet to be explored in the context of a PNF substance use intervention, it seems likely that a three-way interaction might reveal treatment PNF to be most beneficial among high RS sexual minorities in high structural stigma locales, as well as high RS sexual minority participants experiencing greater interpersonal stigma, as these individuals would be most prone to actual ingroup norm conformity. In light of the current study's findings, this is a prediction that warrants future testing with both SMW and SMM.

\section{Did drinkers find the competition acceptable and beneficial?}

Overall, LBQ drinkers completing the post-competition survey found the LezParlay competition to be highly acceptable. Whereas psychological reactance and defensiveness among heavy drinkers often undermine the impact of norms correction in traditional PNF alcohol interventions (e.g., Boyle et al., 2018; Leffingwell et al., 2014), SMW taking part in the evaluation study rated the receipt of detailed results (i.e., PNF) as the most liked aspect of 
LezParlay with no differences in ratings by PNF condition or level of alcohol consumption.

Thus, those receiving less and more health-related results as a function of condition, and those who entered the study as lighter and heavier drinkers similarly liked receiving the PNF delivered 
by LezParlay. There are two potential explanations for these findings. Either heavy drinking SMW lack a tendency toward reactance and defensiveness in the context of alcohol PNF, making them very different from other populations, or more probable, LezParlay's deep structure cultural tailoring, game framing, copresence, chance-based uncertainty, and PNF on multiple topics worked satisfy psychological needs and thereby circumvent negative reactions to treatment PNF as intended.

Most participants (93\%) also reported feeling that they psychologically benefitted from taking part in the competition. Descriptions of benefits reflected social comparison, introspection, and self-confrontation; benefits closely aligned with the goals of traditional PNF (Miller et al., 2016), and therefore, somewhat predictable in the context of a gamified PNF intervention. However, it is notable that college student drinkers taking administered traditional alcohol PNF and questioned about their experience rarely articulate such benefits and more commonly voice skepticism about the authenticity of normative statistics presented (e.g., Hummer, Hatch, \& Davison, 2020; Miller \& Leffingwell, 2013). Thus, benefits in the realms of social comparison, introspection, and self-confrontation, described by LezParlay participants may be attributable to specific game mechanics intended to foster need satisfaction. For instance, social comparison was encouraged in LezParlay through PNF screens but also through scoring and leaderboards. Similarly, benefits related to introspection and self-confrontation suggest that features designed to foster autonomy (i.e., multiple question and feedback topics, chance-based uncertainty, user-submitted questions) likely contributed to PNF across topics not being experienced as controlling, which minimized psychological reactance when viewing healthrelated PNF. It is also likely that LezParlay's copresence features, which allowed actual norms to be transparently derived from the responses of visible peers, worked to minimize actual norm 
skepticism, thereby encouraging social comparison and introspection.

Participants also reported benefits related to community connection and identity strength, stigma reduction, enhanced mood/outlook, and entertainment. Many of these benefits map onto LezParlay's constellation of game mechanics and mix of fun and serious topics, and importantly, stretch beyond those that would be expected in the PNF intervention context. Two varieties of benefits described by participants were unexpected and merit discussion in the context of adjacent research into well-being among SMW and intra-community identity dynamics. First, many benefits suggested that the virtual community of LBQ women created by LezParlay in combination with PNF shattering a myriad of negative stereotypes related to identity, health, relationships, and sexual behavior may have indirectly fostered well-being by increasing community connection and identity strength while decreasing internalized stigma and feelings of isolation. These benefits map onto SDT's satisfaction of relatedness needs and suggest LezParlay may buffer some of the mechanisms (i.e., negative self-schemas, social isolation) by which sexual minority stress has been theorized to "get under the skin" to negatively impact health (Hatzenbuehler, 2009). Thus, although more research is needed, these findings suggest that challenging negative sexual identity-related stereotypes and including virtual community copresence features may also prove fruitful in sexual minority stress informed interventions targeting loneliness/isolation, internalized stigma, and negative self-schemas (e.g., Pachankis et al., 2020; Ratts et al., 2013; Riggle et al., 2014).

Changes in inter-community perceptions was the second unexpected, but interesting, variety of psychological benefit described by a sub-group of participants. That is, several participants reported that learning about the experiences of other sexual minority identity groups improved their perceptions of these groups, with lesbian women indicating that their perceptions 
of bisexual women improved and vice versa. Such benefits are notable in the context of research into LBQ community dynamics, which has documented biphobia among lesbians who view bisexual women through stereotypic tropes (e.g., promiscuous, likely to cheat in relationships; Feinstein et al., 2017; Weiss, 2004; Welzer-Lang, 2008) and similarly documented negative views of lesbians among queer and bisexual women who view these community members as too rigid and conservative in their gender and identity politics (e.g., man-hating and transexclusionary radical feminist lesbian stereotypes; Morris, 2016; Sexton et al., 2018). Whereas the majority of PNF screens in this version of LezParlay focused on the player's own sexual identity and age-group, these reported benefits suggest that expanding the presentation of actual norms for the behaviors and experiences of adjacent sexual identity peers (in addition to one's own sexual identity group) in future versions of LezParlay may work to reduce the intra-community stigma, tension, and exclusionary forces that have been documented in female sexual minority communities and found to negatively impact well-being (e.g., Feinstein et al., 2017; Ghabrial, 2019; Morris, 2016; Weiss, 2004; Welzer-Lang, 2008).

In a review paper highlighting the great need for alcohol interventions and treatment programs for sexual and gender minorities, Talley (2013) speculated that culturally tailored interventions for LGBTQ populations might confer participants additional wellness benefits beyond core intervention content such as identity positivity, community connection, and social support. Both benefit insights and feature requests submitted by participants provide support for this speculation in the context of LezParlay. For instance, many of the players who reported benefits in the categories of identity strength/community connection suggested ways in which these benefits might be maximized in future versions of the competition, for example, via messaging, chat, and/or forum features that would allow for greater interactivity and support 
between players. In general, requests reflected that participants desired no major fundamental changes to the competition, but rather, they wanted more of what the initial version of the competition offered combined with a sleeker user experience; requesting for example, a native app, more frequent rounds with results delivered faster, greater ease of referring friends, additional questions and results comparing sub-groups of SMW, and the connection of detailed results to additional informational posts and resources, etc.

In response to the feature requests submitted by participants, the next version of the LezParlay competition is likely to take the form of a native smartphone application and feature weekly rounds with cumulative monthly scoring and prizes. Additional interactivity features will likely allow users to direct message/chat with one another, post to a public newsfeed, and both view and comment on topic-specific blog posts and resources and blog posts following completion of each round. In addition, as several players described benefits related to seeing results for other sexual identity groups beyond their own and others requested that some rounds/questions be specific to race, gender presentation, or geographic region (country/region), future versions may also include greater variability in format. For example, increasing the frequency of rounds from monthly to weekly would allow health-related questions and treatment PNF to be delivered over time rather than all at once. In rounds featuring treatment questions, all questions might have the sexual identity and age-specific reference groups consistent with the format in this initial study. However, additional weekly rounds might take challenge users to guess and wager points on the behaviors and experiences of sexual identity groups other than their own and/or increase reference group specificity to include additional characteristics (race, gender presentation, geographic location) requested by participants.

All considered, this trial's findings were quite encouraging. Feasibility data and 
participant insights together illuminated a number of ways the user experience and larger competition might be improved to maximize engagement and psychological benefits. Efficacy findings, in turn, suggested a number of needed improvements to the study design and measures that could increase the value and rigor of future evaluation efforts. The next sections detail these considerations, provide an overview of the next steps for LezParlay intervention development, and present several broader directions for future research.

\subsection{Limitations \& Future Directions}

The current hybrid trial included a number of cost-cutting methodological limitations acknowledged prior to study launch as well as several unanticipated limitations which emerged later in data collection and analysis phases. Findings from the current study should be considered in light of both types of limitations. Each is introduced before detailing how these issues might be simultaneously addressed through a larger, more sophisticated trial.

The first notable limitation relates to the technology used in the current trial. That is, the LezParlay app tested in this study was a "minimally viable product" taking the form of an extremely low-cost progressive web application designed and coded entirely by the researcher. As such, efficacy and feasibility findings from the present study may not generalize to more (or less) technologically sophisticated version of the competition. For instance, drinkers in the evaluation study commonly requested that future versions of LezParlay take the form of a native smartphone application, which may be more desirable and better equipped to sustain play among SMW. Native app versions of LezParlay may also greatly benefit the presentation of game mechanics, potentially increasing attention to PNF, and thereby having greater behavioral impact. However, a move to this technology may also present barriers to participation among sub-groups of SMW (older SMW not as familiar with Apple/Android app stores, lower 
socioeconomic status SMW who may lack smartphones altogether, SMW with smartphones paid for by their employers and used primarily for work purposes). Thus, it will be important for future research to examine potential differences in feasibility and efficacy associated with application technology.

Another limitation pertains to the current study's organic assessment of baseline and follow-up alcohol outcomes as well as some moderators (i.e., interpersonal stigma) within rounds of the competition. In some ways, this was a major strength in that organic assessments eliminated the demand characteristics that too often plague transparent PNF intervention studies and reduced the costs associated with survey-based follow-up. However, in-competition assessment meant that key constructs could only be assessed by a few items, and further, the language of items could not be too formal or clinical in tone. Although the QFM (Baer, 1993) fit well in this regard as a short, validated, clinically meaningful alcohol use measure, it would have also been beneficial to collect data using additional, validated survey measures to more formally assess drinks per week (i.e., Daily Drinking Questionnaire), negative alcohol-related consequences, and screen for alcohol use disorder (i.e., Alcohol Use Disorders Identification Test).

The current study's single, two-month follow-up assessment and limited evaluation study sample size represent additional limitations. Although consistent with the duration of follow-up common in the standalone PNF intervention literature (e.g., Earle et al., 2018, Pedersen et al., 2017), it will be important for future work to include additional, longer term follow-up assessments (i.e., 6, 12 months post-intervention) in order to examine the extent to which the treatment effects observed in the current study persist or fade over time. In traditional PNF intervention studies with other populations, effects have generally been found to fade rather 
quickly following a single dose of alcohol PNF with a minority of studies exhibiting meaningful effects 6-12 months post-intervention (see Dotson et al., 2015 for a review). In the event that treatment effects are observed to fade similarly among SMW taking part in LezParlay, it will be important for future work to consider how aspects of the competition might be modified or expanded to bolster and extend treatment effects. Further, although the sample size in the evaluation study ensured sufficient statistical power for testing hypothesizes and all two-way interactions, this study lacked sufficient power to test exploratory three-way interactions, potentially preventing identification of additional sub-groups of participants for whom alcohol+coping PNF was more beneficial than alcohol PNF alone. For instance, H3b and H4b, which predicted that alcohol+coping PNF would be more effective than alcohol only PNF among participants reporting greater interpersonal stigma exposure as well as those living in high structural stigma communities, was not supported in this study. However, it seems plausible that receipt of alcohol+coping PNF (relative to alcohol only PNF) may have been increasingly effective in reducing drinking among SMW who were exposed to greater interpersonal and/or structural stigma and entered the study as heavy drinkers and/or frequently drinking to cope with stress and stigma (i.e., condition*stigma exposure*baseline drinking and/or condition*stigma exposure*proportion of time use alcohol to cope). It will be important for future research to include larger samples of drinkers to ensure that these interactions can be tested.

Additional limitations emerged during data collection and analysis phases of the trial. The first was a consequence of the unexpectedly large number of players $(N=2,667)$ who signed up to take part in LezParlay during the 8-month competition period, which more than doubled the number expected (target $N=1200)$. Although the large number of sign-ups and web app traffic in the absence of traditional study incentives suggest the potential scalability of this 
approach, all efforts to sustain play and re-engage SMW not taking part in the evaluation study were abandoned once the number of sign-ups grew too large to handle manually. This tipping point occurred mid-competition and thus was problematic in that it limited the conclusions that could be drawn about round play and app engagement among SMW not taking part in the evaluation study. Thus, future versions of the app should include fully automated notification systems for onboarding messages, round reminders, and detailed results delivery, in lieu of the manual email and text-message based approach that became unmanageable in this study.

At the point of analysis, it also became clear that although all specific hypotheses could be addressed, the study design was short-sighted in two major ways. First, it assumed that healthful changes in coping behaviors would be reflected in decreases in global alcohol use outcomes. Because coping norms and behaviors were only assessed a single time after treatment PNF on alcohol use had already been delivered, it was not possible to directly examine whether correcting coping norms impacted coping behaviors or determine the extent to which coping behaviors were reflected in global drinking outcomes. In light of this issue, future research should assess coping norms and behaviors at the same points in time as alcohol use norms and behaviors and evaluate the extent to which correcting coping norms impacts subsequent coping behaviors. Second, whereas feedback collected from study participants suggested that LezParlay's social media-inspired copresence features and the correction of negative stereotyperelated norms via PNF may have carried psychological benefits beyond treatment PNF (i.e., reducing identity-related stigma, increasing feelings of community connection, decreasing intracommunity divisiveness), the current study did not quantitatively assess these psychological outcomes. This is problematic given the current study design, which included a fully active control condition that only differed from treatment conditions in the topics on which PNF was 
delivered in Rounds 3 and 4. Thus, participants in both treatment and control conditions similarly stood to benefit from the copresence features and the correction of negative stereotype-related norms via PNF in other rounds, which in turn, may have decreased drinking to some degree across conditions. Thus, it is important to emphasize that estimates of effect size for treatment conditions (relative to control) are specific to the PNF delivered in intervention rounds and do not speak to the potential positive effects of LezParlay’s deep structure cultural adaptations or game mechanics. To more fully examine the extent to which taking part in LezParlay benefits SMW psychologically and reduces drinking, it will be necessary for future trials to also include a waitlist assessment only control condition.

The next step in this program of research is to conduct a substantially larger and sophisticated hybrid trial that addresses the current study's limitations. This research will feature both waitlist control and active control conditions in order to directly investigate potential mental health and behavioral benefits associated with non-treatment, negative stereotype challenging PNF, and virtual community participation. This study will also shift to a full repeated-measures survey assessment format, with baseline surveys completed prior to app engagement and additional surveys completed at 3, 6, 9, and 12 months post-baseline. Validated survey measures will more completely assess alcohol-related and additional outcomes (e.g., coping behaviors, health behaviors, depression, anxiety, sense of belongingness, loneliness, internalized stigma, collective self-esteem), as well as mediators (e.g., drinking/coping norms, negative stereotype related norms), and potential moderators of efficacy (e.g., rejection sensitivity, recent and historical interpersonal stigma experiences varying in severity, etc.).

To maximize LezParlay’s acceptability, psychological benefits, and efficacy as an intervention strategy, future versions of the LezParlay competition will also draw on additional 
research and theory in an effort to expand intervention content. For instance, whereas the current version of the competition and present trial exclusively focused on correcting descriptive norms, future research will expand the competition to include additional types of norms and evaluate the extent to which additional norm components further reduce drinking among SMW. Specifically, future research may seek to evaluate the utility of include judgment-based injunctive feedback component that builds on promising pilot findings among college students (Earle et al., 2018) but is further informed and extended by deviance regulation theory (DRT; Blanton \& Christie, 2003). DRT contends that conflicting needs for "standing out" and "fitting in" with social groups motivate people to deviate from group norms in socially attractive ways and, at the same time, avoid socially unattractive behaviors that stray from group norms. According to this theory, to reduce heavy drinking, it would be beneficial to couple the presentation of a descriptive norm demonstrating that heavy consumption is not normative (as done via alcohol PNF in the current study) with additional data demonstrating that fellow SMW rate heavy drinking peers quite negatively (i.e., an ought injunctive norm). Conversely, to encourage healthy stress coping behaviors like the use of meditation and relaxation techniques, which PNF revealed in this study to be underestimated descriptively but a behavior only engaged in by roughly half of group members, it would be more beneficial to present data demonstrating that group members rate SMW engaging in this behavior very favorably (i.e., an ideal injunctive norm). Future research will examine whether this type of peer feedback can be organically produced in the competition through an additional component in which players can earn bonus points for snap judgments, or quick ratings, of the hypothetical behaviors and attitudes of LBQ peers (e.g., How do you rate a lesbian in her 30s who drinks 7 days per week?).

The move to a more frequent weekly round format, wherein treatment topics are 
addressed via individual questions and PNF more sporadically over time, and some key questions are reassessed at later points in time, will also provide a natural environment for examining the utility of dynamic, or "trending" norms (Sparkman \& Walton, 2017, 2019) in the gamified PNF context. Whereas PNF alcohol interventions have traditionally focused on actual static descriptive norms that provide information about how often and how much group members are drinking at a single point in time (e.g., the typical lesbian player in her 20s drinks 3 days per week), dynamic norms focus on how group members' alcohol consumption is changing or has changed over time (e.g., Over the past month, $65 \%$ of lesbians in their 30s decreased their number drinking days per week). Recent research has demonstrated that communicating information specific to descriptive dynamic norms can positively impact behavior change beyond the effects associated with static descriptive norms information alone, and this remains true even if the dynamic norm does not communicate that a majority of the group has changed (Sparkman \& Walton, 2017). Further, findings suggest that dynamic norms impact behavior by increasing collective self-efficacy (i.e., decreasing your drinking IS possible) and demonstrating that change is both valued by fellow group members and compatible with the group's social identity (Sparkman \& Walton, 2019). Future research will examine ways in which dynamic norms information could be incorporated into a multi-round, gamified PNF competition to potentially boost treatment effects.

Although the current norms-focused intervention was effective in reducing alcohol consumption and negative consequences, future research will also examine whether treatment effects can be enhanced and extended through the inclusion of additional intervention components. For instance, the reasoned action approach (Fishbein \& Ajzen, 2011) holds that in addition to perceived descriptive and injunctive norms, perceived behavioral control is a 
critically important antecedent to behavior change. This suggests that LezParlay's drinking reduction effects might be increased substantially if the intervention included additional skillbuilding components designed to increase perceived capacity for reducing drinking and engaging in adaptive coping strategies. One way that this might be increased among PNF-motivated drinkers would be to provide external links to more intensive digital interventions like those being developed by Pachankis et al. (2020) and Bush et al. (2019) for SMW seeking treatment for alcohol use disorder. Alternatively, additional alcohol intervention components and resources designed to increase capacity for behavior change could also be integrated into the competition app. Building on Screening, Brief Intervention, and Referral to Treatment Programs (SBIRT; Babor et al., 2007), PNF could serve as the brief intervention with PNF screens funneling players to appropriate resources, interventions, and local treatment programs based on their round responses to questions about alcohol consumption and coping.

Given the high engagement and positive feedback received from SMW in the current study and the additional health behavior norms widely misperceived in non-intervention rounds, a related direction for future research is to examine such a competition's utility as an initial step in a broader multi-component health intervention for SMW. That is, health-related PNF delivered within the competition could work to increase SMWs' motivation for healthful behavior change (e.g., exercising, quitting smoking) before connecting SMW to more intensive, culturally tailored, capacity building interventions targeting these behaviors. In this way, the LezParlay competition could help resolve some of the barriers to engagement and implementation that have challenged intensive health behavior interventions for this population in the past (e.g., Rizer et al., 2015), while serving as a fun, central hub for LBQ community connection, visibility, and wellness. 
A final priority direction for future research centers on investigating additional sources of norm misperception in the domains of substance use and health behaviors among SMW. While previous work has linked frequency of lesbian bar patronage and social media use to perceptions of lesbian drinking norms (Boyle et al., 2016; Boyle, LaBrie, et al. 2020), participants also alluded to negative, stereotypic portrayals of LBQ women in television shows and movies as potential sources of misperception in describing benefits associated with competition participation. Previous research has documented stereotypic portrayals of LGBTQ characters on primetime television shows for mainstream audiences (Fisher, Hill, Grube, Gruber, 2007; McInroy \& Craig, 2017; Raley \& Lucas, 2006); however, very little research has examined how LBQ health behaviors and relationship functioning are portrayed more specifically on popular premium cable series with predominantly queer female casts, writers, and producers (i.e., ingroup television programs). As these shows tend to glamorize queer female drinking cultures and are created by ingroup LBQ community members, they may play a substantial but understudied role in communicating LBQ alcohol use and health behavior norms. Thus, future research will examine the degree to which program exposure and watching habits are associated with perceptions of LBQ health behavior norms. If viewership is found to predict perceived norms, efforts to promote the LezParlay competition could be expanded to better reach and appeal to the audiences of such programs (e.g., targeting audiences via social media ad campaigns, pre and post-air advertisements, getting cast members involved in competition promotion). In addition to these immediate next steps for LezParlay research, findings from the present study also carry several broader implications for theory, research, and intervention development. 


\subsection{Broader Implications for Research and Practice}

The current study's findings, although specific to SMW, should encourage researchers to investigate perceptions of peer norms as antecedents to sexual-orientation-based health disparities and consider perceived norms for sexual minority peer health-risk behaviors as potential intervention targets. Parallel to drinking norm findings among SMW, numerous studies have found peer substance use norms to be both over-estimated and strong predictors of substance use among both gay and bisexual men and LGBQ youth (see Boyle et al., 2020 for a review). However, PNF interventions have rarely been considered as potential strategies for reducing problematic substance use in these populations. Further, normative constructs remain vague (Lick et al., 2013) or oversimplified (Hatzenbuehler, 2009) in expanded theoretical models of sexual minority stress and health-risk behavior. For instance, Hatzenbuehler's (2009) expanded psychological mediator model of sexual minority stress positions permissive LGB community norms as a social-interpersonal mediator of the relationship between distal sexual minority stressors (which encompass the interpersonal stigma experiences assessed in this study) and health behavior. However, findings from this study emphasize the need for distinction between perceived and actual LGB peer norms, suggest that perceptions of these norms may be elevated by specific types of distal stressors (i.e., violence and harassment due to sexual minority status), and reveal that these same stressors may increase conformity to perceived LGB norms. Thus, these findings point to a clear need for additional research assessing sexual minority stigma experiences and perceptions of sexual minority peers' health behavior (tobacco use, illicit drug use, condom use, etc.) both in relation to one another and as potentially interacting predictors of individuals' own health behavior. If the relationships observed in this study can be replicated for other sexual minority sub-groups, theoretical models should be updated to reflect 
more precise operationalizations of norms and relationships between stressors, perceived norms, and health behavior.

The present research also makes a novel contribution to the larger SNT literature by demonstrating how a PNF intervention can be packaged as a social competition designed to challenge negative stereotypes about a stigmatized minority group in order to reach, engage, and provide group members additional psychological benefits that extend beyond treatment PNF. Notably, this is the first PNF intervention study to connect the exaggerated perceptions of group norms at the heart of SNT to stereotypes, defined as the over-generalized beliefs about the characteristics of social groups (e.g., Devine, 1989; Vinacke, 1957), and self-stereotyping, defined as the process of conforming to stereotypes about one's own group (e.g., Hogg \& Turner, 1987). As similar interventions could easily be designed to counter identity-related stigma and negative stereotypes in other minority groups, future research is needed to examine the reliability with which stigmatized group members overestimate negative stereotypic behaviors and attitudes among ingroup peers and further investigate the psychological impacts of correcting these misperceptions.

An additional direction for future research is also suggested by participant descriptions of improved perceptions and attitudes toward sexual identity out-groups following PNF. To date, PNF interventions have exclusively focused on correcting overexaggerated perceptions of ingroup members' behaviors and attitudes. Although more research is needed, findings from the current study suggest that normative feedback designed to correct overexaggerated perceptions of out-groups may also help to lessen negative perceptions of these groups. In light of the extremist identity politics and online echo chambers that currently plague American society, the potential usefulness of a gamified, normative feedback competition designed to correct 
exaggerated inter-group perceptions also merits further exploration. Whereas in the current study lesbian, bisexual, and queer women all shared a meaningful superordinate sexual minority social identity, an important question to be addressed in future work is whether correcting inter-group perceptions of behavioral and attitudinal norms benefits inter-group perceptions or relations when such a clear and meaningful superordinate social identity is not shared between groups (e.g., the superordinate identity American among registered republicans and democrats).

Findings also carry implications for future gamified health intervention development and evaluation. In the context of COVID-19 and our socially distanced world, there has never been a time of greater need for cost-effective, palatable, digital interventions able to simultaneously engage, entertain, and bolster individuals' sense of social connection whilst effectively motivating health behavior change. The current research demonstrates how competition framing and game mechanics informed by SDT's basic needs for competence, relatedness, and autonomy can be injected into the very fabric of an evidence-based intervention to make traditionally dry participant tasks enjoyable and psychologically beneficial. In particular, participants’ descriptions of benefits and feature requests underscore the importance of relatedness needs in the digital health context and highlight the utility of social media-inspired copresence features for creating virtual user communities. Further, the Type I Hybrid Effectiveness-Implementation design employed herein allowed RCT-based efficacy data and feasibility data to be gathered simultaneously, providing valuable insights into sign-ups in the absence of traditional study incentives, engagement with different areas of the app, and participant feedback on the gamified intervention experience. This hybrid trial design represents a methodology that may be uniquely valuable for gamified health interventionists seeking to cost-effectively evaluate efficacy and feasibility early on in the intervention development process. 
Most broadly, findings from this study should encourage interventionists to carefully consider the manner in which evidence-based interventions for nonclinical populations are packaged for digital dissemination. Although potentials for increased cost-efficacy and scalability have led to great enthusiasm and much federal funding for digital health interventions (Hansen \& Scheier, 2019), recent reviews have revealed that the majority of apps designed to reduce or prevent health-risk behaviors in nonclinical populations (e.g., reduce alcohol use, sedentary behavior, unsafe sex) are not widely downloaded or used (Kohl, Crutzen, \& de Vries, 2013; Szinay, Jones, Chadborn, Brown, \& Naughton, 2020). Further, even in studies where intended users are recruited and paid to engage with such apps, only a minority have been found to meaningfully change target behaviors or cognitions (e.g., Colbert, Thornton, \& Richmond, 2020; Milne-Ives et al. 2020). Social psychological research has revealed two facts likely at the root of these issues. Many individuals who engage in health-risk behaviors view these behaviors as normative and, therefore, not problematic (e.g., Miller \& Prentice, 2016). Others are aware that they are putting themselves in harms' way (e.g., smokers, heavy drinkers) yet lack sufficient motivation to change their behavior (e.g., Kelly, Zyanski, \& Alemagno, 1991). For both groups, digital health behavior change apps transparently focused on reducing a stigmatized behavior are unlikely to be appealing or interesting, and much like traditional PNF alcohol interventions, may arouse reactance and defensiveness (e.g., Brehm, 1966; Goldenberg \& Arndt, 2008).

The present research illustrates one way in which these issues might be avoided; that is, by purposely removing a digital intervention's explicit focus on a target behavior and incorporating framing, content, or features of high interest or utility among target users to foster psychological need satisfaction. In the current intervention, culturally tailored competition framing and digital game mechanics worked to camouflage the target behavior (i.e., alcohol use) 
and make the evidence-based behavior change strategy (correcting norms via PNF) highly credible and palatable. As could be done in other digital health interventions for nonclinical populations, all participant tasks, experiences, and potential risks and benefits were fully and transparently communicated in lay terms, without stigmatizing target behaviors or labeling the activity as an intervention. This approach, wherein language related to interventions, target behaviors, and key psychological processes are omitted from participant materials is also consistent with emerging work on the development of WISE interventions for personal and social problems (Walton, 2014; Walton, \& Wilson, 2018; Walton \& Yeager, 2020). A major tenant of the WISE perspective is that intervention formats and delivery contexts are critically important. To be maximally effective, both the way in which an intervention is delivered and context for delivery should be strategically aligned to the way of thinking encouraged by the intervention. For instance, in LezParlay SMW learned that they over-estimated LBQ drinking norms in the context of a game in which they also learned that they similarly misperceived a wide variety of other stereotypic behaviors among LBQ peers. As a whole, this context likely reduced the threat associated with alcohol PNF while further reinforcing the SNT-consistent idea that LBQ peers engage in negative and risky behaviors less than assumed. The WISE perspective would contend that this broader lesson is likely to be internalized and attributed to the self rather than an external "intervention", and therefore, should lead participants to question their LBQspecific perceptual biases and be less prone to conform to norms that put their health and wellbeing at risk in the future. To date, the budding literatures on WISE psychological intervention development, intervention gamification, and deep structure cultural tailoring have remained separate and discipline-specific (i.e. psychology for the former, public health for the latter two). However, as the current research reveals one context in which gamification and deep structure 
cultural adaptations may support WISE intervention goals, these findings encourage further exploration of ways in which these perspectives may be integrated to benefit the efficacy, engagement, and scalability of digital health interventions for nonclinical populations.

\subsection{Conclusion}

As an initial step in a larger program of research, findings from this study support the feasibility and efficacy of a gamified PNF alcohol intervention for SMW, thereby narrowing costly disparities in alcohol intervention research and practice. Although additional research is needed, these initial results demonstrate how deep structure cultural tailoring and game mechanics can be meaningfully injected into a PNF intervention in order to attract, engage, and potentially extend additional psychological benefits to members of a hard-to-reach, stigmatized, health disparity population. Findings also suggest that perceived sexual minority-specific norms and some sexual minority stigma experiences may be more related and interactive than currently positioned in the literature - in this study, making treatment PNF increasingly risk-reducing among SMW experiencing greater violence and harassment due to sexual minority status. All considered, findings underscore the potential utility of PNF intervention components for sexual minority populations and highlight the need for additional research fully investigating the interplay between different types of perceived sexual minority-specific norms (i.e., descriptive, injunctive, static, dynamic), sexual minority stressors, stigma-related processes, and individual difference variables in the context of substance use and other health-risk behaviors. More broadly, this research advances several compelling directions for future PNF intervention development, demonstrates the efficiency of hybrid feasibility/efficacy trial designs for evaluating digital health interventions, and illuminates the potential utility of incognito digital health intervention formats for nonclinical populations. 


\section{Appendix A}

Recruitment \& Promotional Materials 


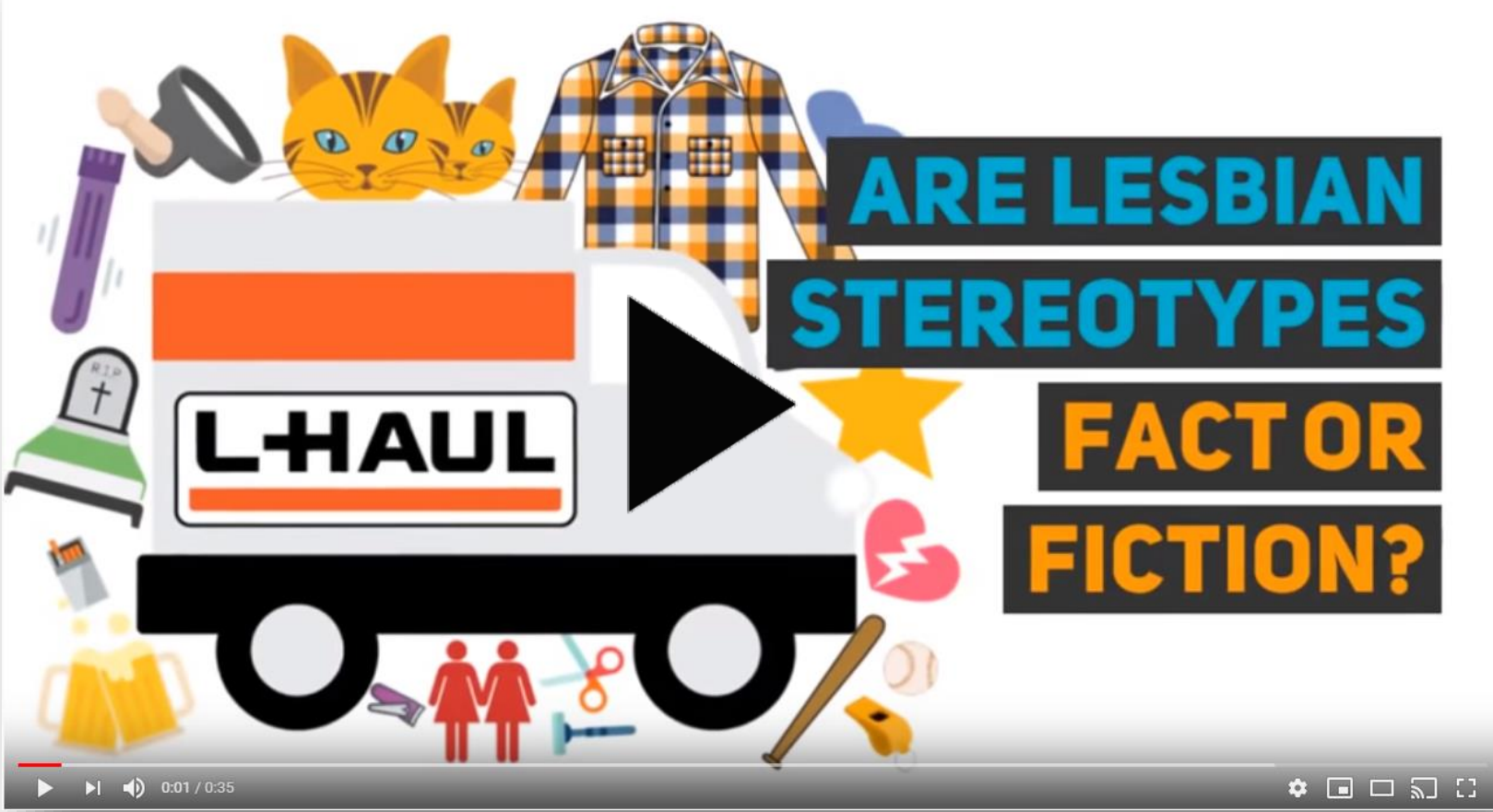

Figure A1. Lesbian-identity tailored HER Social App campaign promo video pinned to the top of newsfeed area. Viewable at https://www.youtube.com/watch?v=x9Y7oobC1Zw

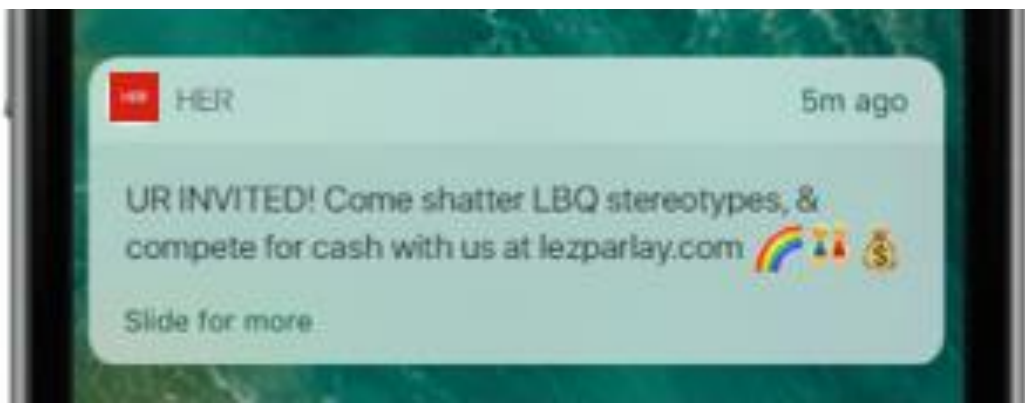

Figure A2. HER Social App banner smartphone notification received by users in Los Angeles, New York City, and Atlanta 


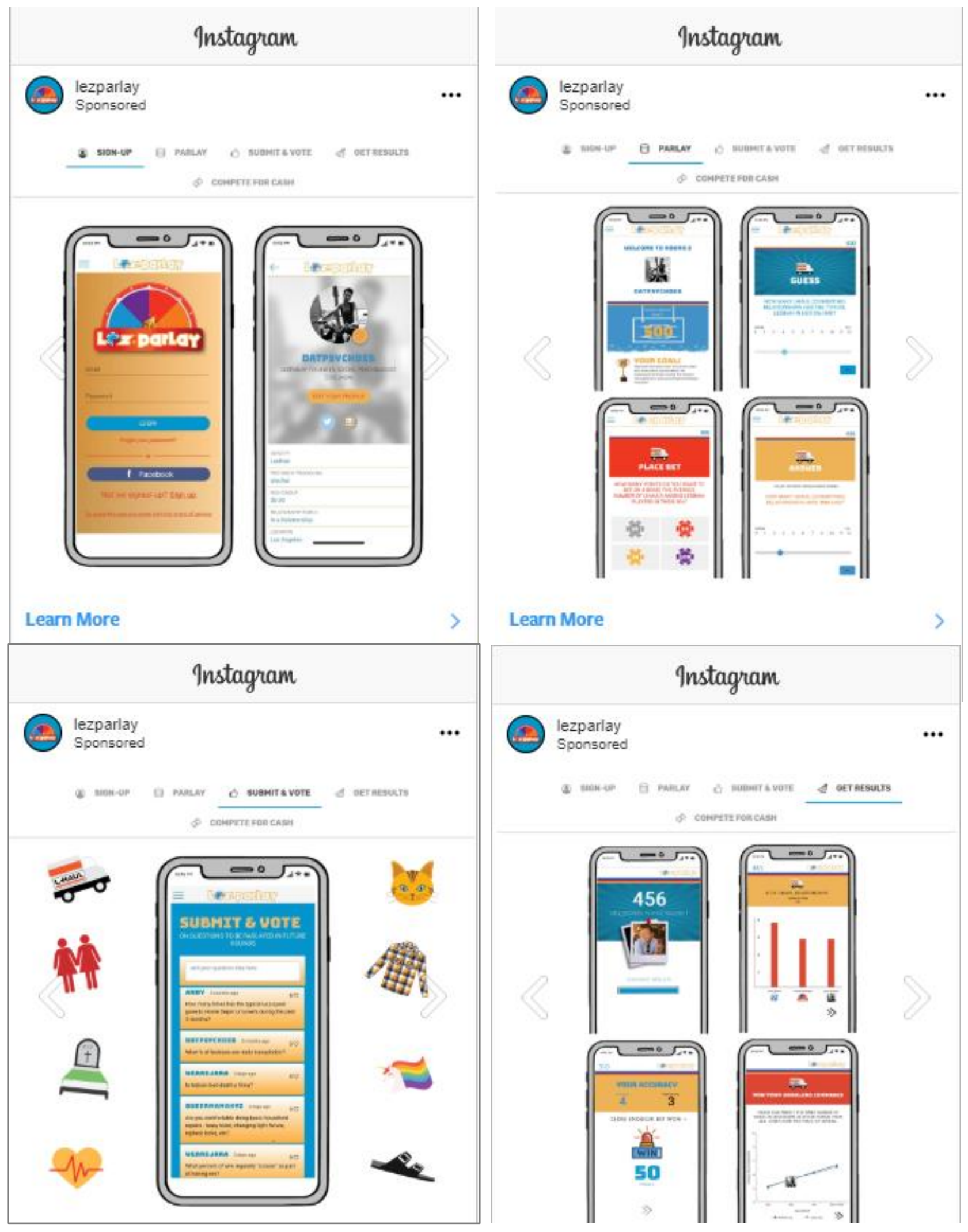

Figure A3. LezParlay's most clicked multi-image carousel ad run on Facebook and Instagram 
Find out how your health, drinking, and relationship history really compare to other women your age while increasing LBQ visibility, challenging stereotypes, \& competing for cash in our online LezParlay competition! IT'S FREE

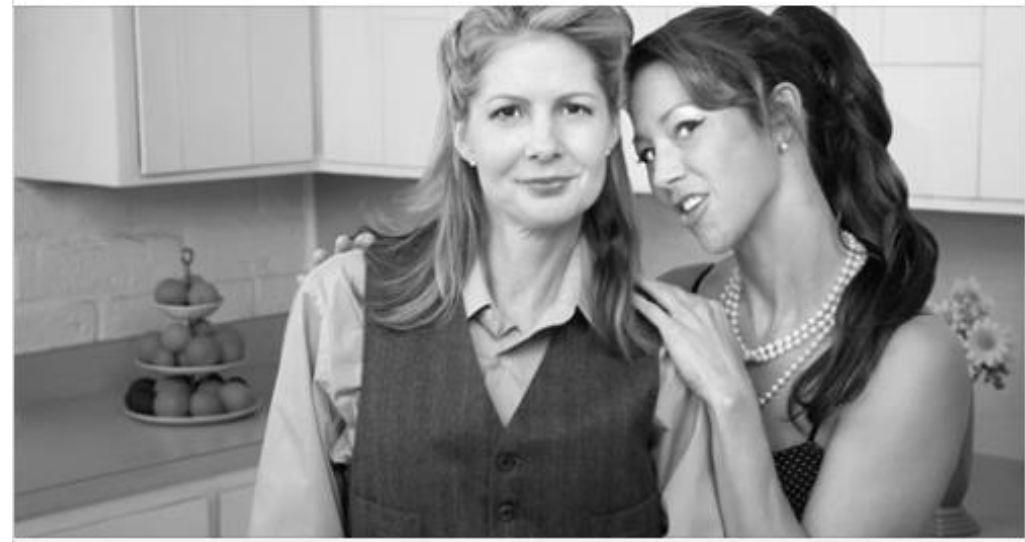

LEZPARLAYLA.COM

Compete against LBQ women in their 40 s \& 50 s

Learn More cats. lesbian bed death. flannel shirts. co-dependence....

@ Like

Comment

$\Rightarrow$ Share

LezParlay

Sponsored *

Got 2 minutes? Round 2 of the competition closes soon and $\$ 300$ is up for grabs... 800 LBQ players and growing, you don't wanna miss this!

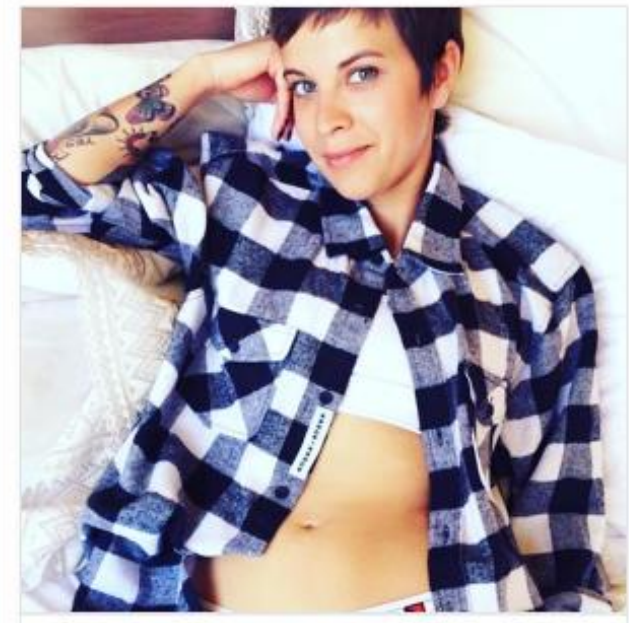

tats, flannel, \& gold stars

Sign Up

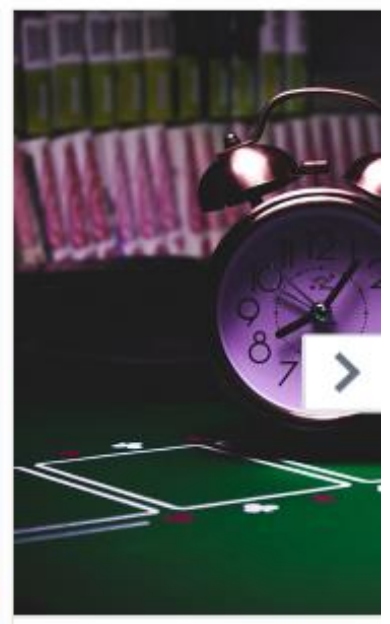

get your bets in before it's toc

$\Rightarrow$ Share

Figure A4. Additional ads run concurrently on Facebook and Instagram. 
Stereotype Challenge | for Lesbian, Bi, \& Queer Women

Ad www.lezparlayla.com

Test your perceptions. Find out how your health, relationships, \& sex life really compare. 4 age-groups:

Play in your 20 s, 30 s, 40 s, or 50 s. $\$ 10,000$ in cash prizes $\& 100 \%$ free!

Learn About the Cause

Learn more

Find out why we created this
Find Out How it Works

Get the details

View the FAQs

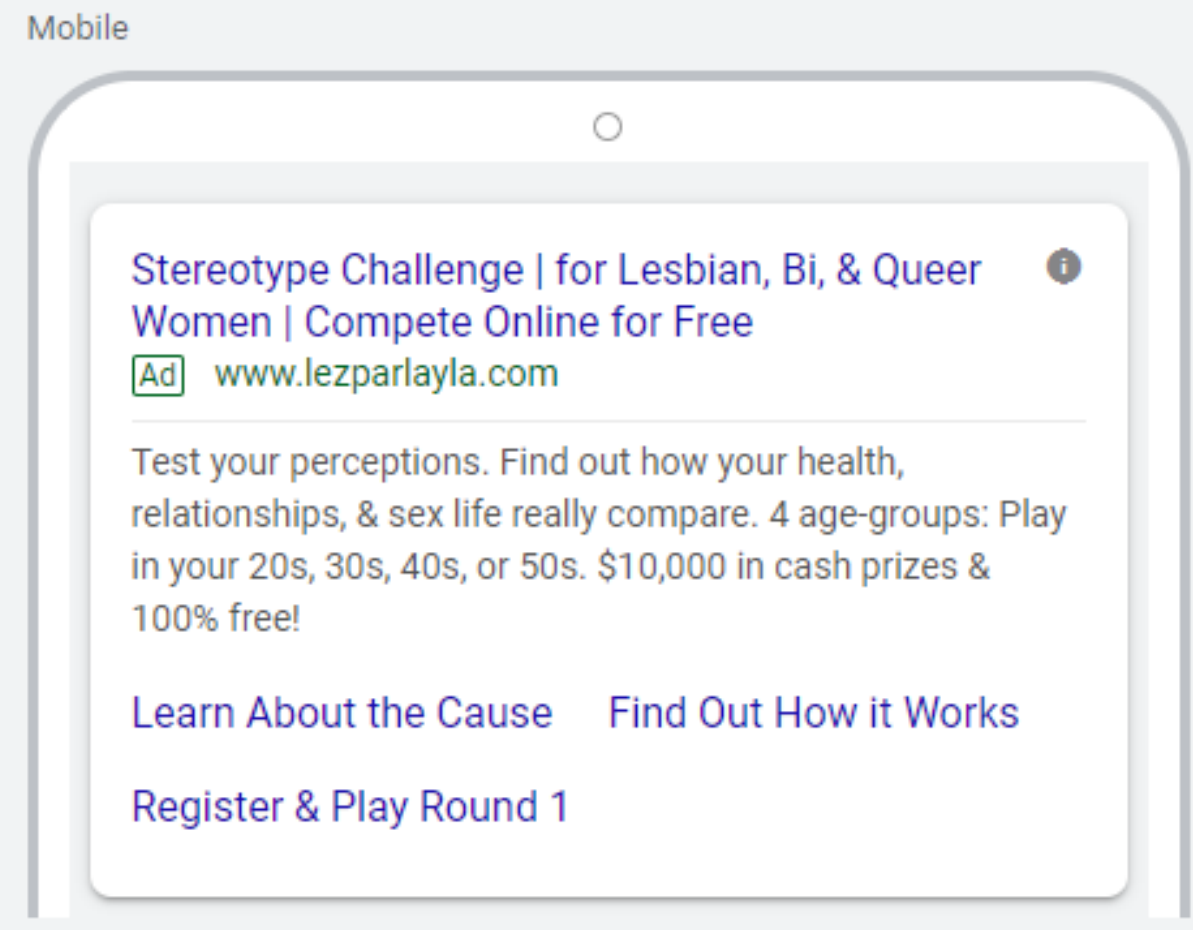

Figure A5. Google Search Ads targeting searches of terms and phrases including (but not limited to) "lesbian chat", "lesbian dating site" "queer dating" "bisexual dating" "LGBTQ pride events" "queer chat" "lesbian bar" "queer nightclub" "lesbian party" "queer party" 


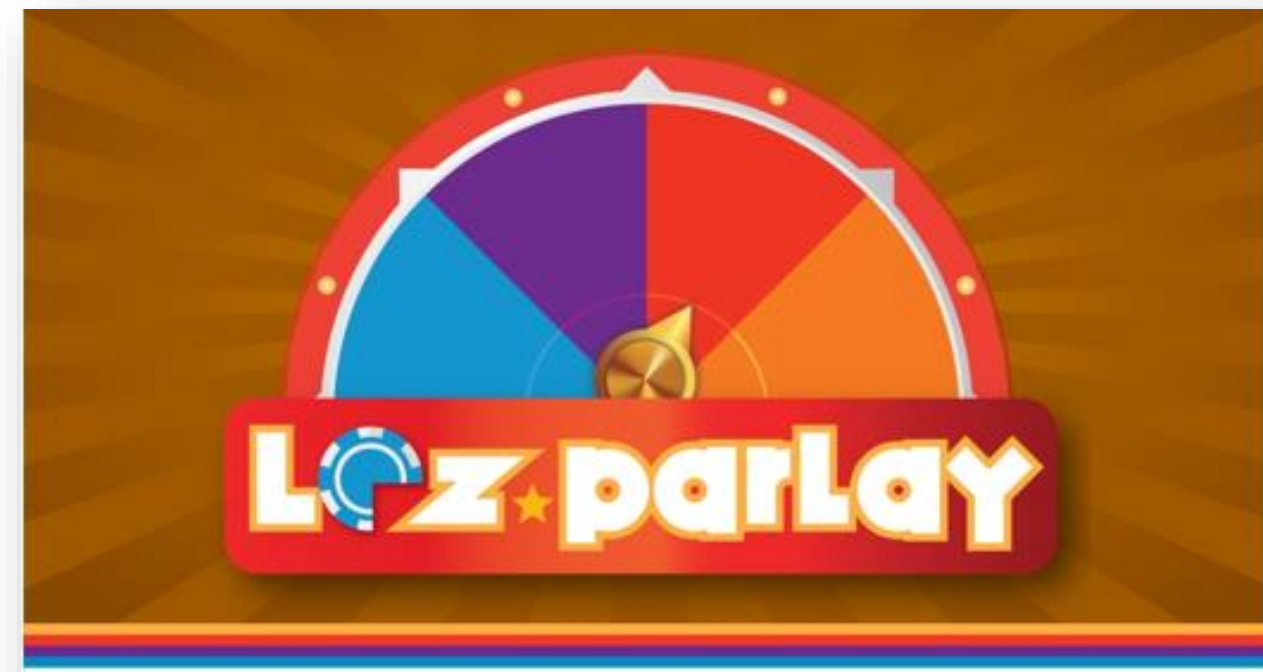

\section{A FREE DICITAL COMPETITION FOR LESBIAN, BISEXUAL, \& OUEER WOMEN!}

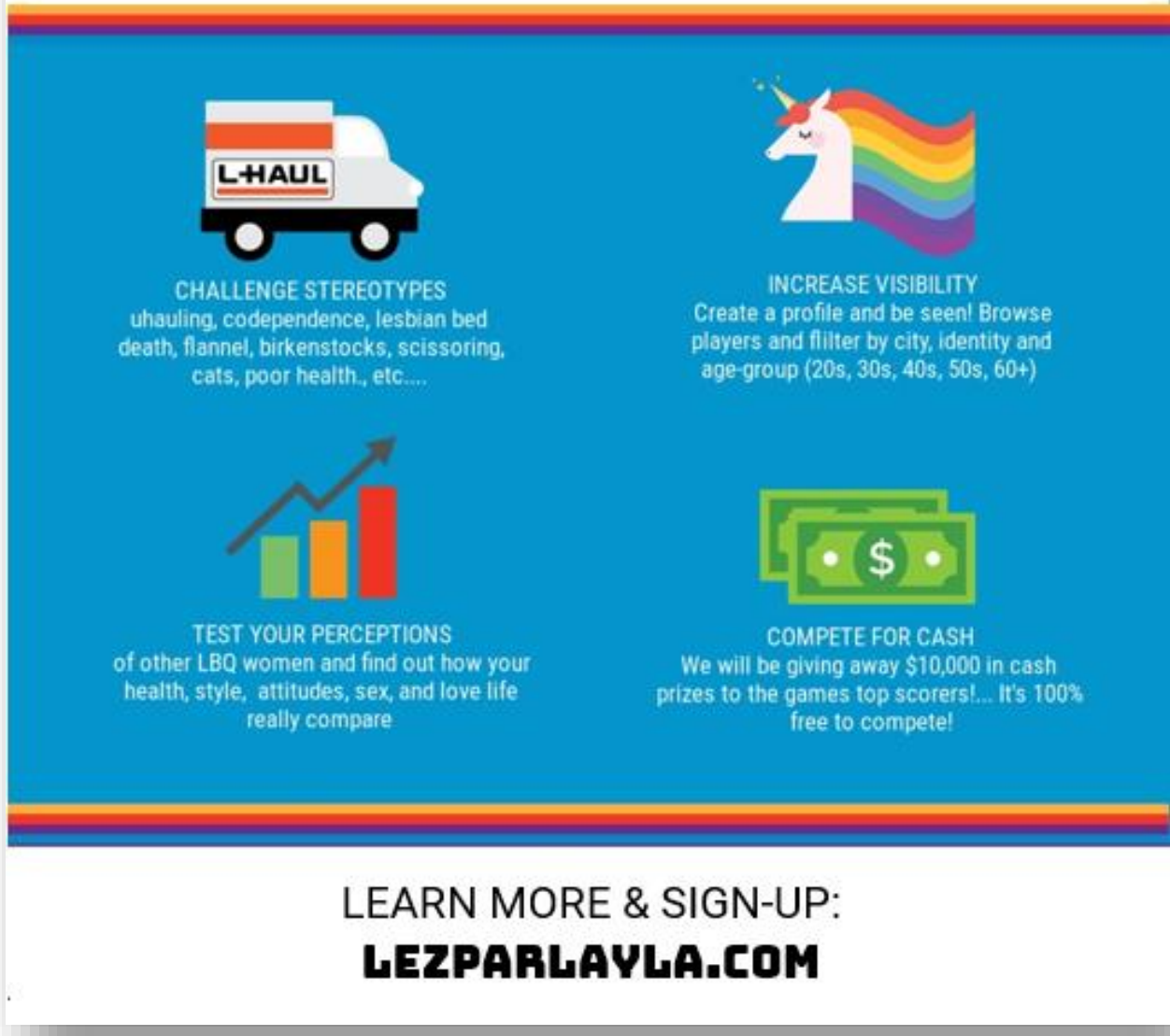

Figure A6. Flyer distributed at lesbian and queer events in Los Angeles, CA along with promotional branded items (chapsticks, coffee mugs, temporary tattoos) 


\section{Appendix B}

Consent Forms \& Evaluation Study Invite 


\section{Competition Terms of Service \\ (basic consent accepted prior to creating a user account in the LezParlay web app)}

\section{TERMS OF SERUICE}

\section{ELIGIBILITY}

To PARLAY you must identify as a lesbian, bisexual, or queer woman, be 21 years of age or older, and presently reside in the United States.

\section{DATA USE, PRIVACY, \& CONFIDENTIALITY}

The LezParlay web application collects some personal data from its users. This information, detailed below, allows us assess interest from lesbian, bisexual, and queer women, understand app usage, and provide you with a smooth, efficient, and customized app experience. Personal data includes Facebook authentication data, demographic and other personally identifiable information that you voluntarily give to us when electing to create an account to participate in the LezParlay competition. When you register, you will sign-up with your email address and password or use your existing Facebook credentials. The only Facebook account data to be accessed by LezParlay is your email address and profile photo, and you will be prompted to grant or deny LezParlay permission to access these items. Next, you will create your LezParlay profile which includes your nickname/initials, age-group, sexual identity, and relationship status. Within the app, you will be represented by an optional photo and your public profile information (sexual identity, age-group, \& relationship status will be visible to other authenticated players in the BROWSE area of the application, and the photos and public profiles of other users will similarly be visible to you in this area). However, you may choose to change your profile photo or delete it in the event you would prefer to remain faceless within the game by editing your account settings. You will also be prompted to provide a verifiable mobile phone number and email address when you create an account, however your contact information will remain private and will NOT be visible to other users. The app only uses this information to send you private results and prize notifications. You may opt out of receiving email and text notifications from LezParlay at any time, without penalty. To opt out of emails use the unsubscribe link provided. To opt out of text notifications simply reply too any text from LezParlay with "STOP". NONE of the information you provide at registration will be linked with your responses to competition questions or bets. Instead, your initial login at sign-up and the mobile phone number you provide will produce a unique code in our system which we will then use to link up your question responses, bets, and points across rounds. In sum, the application uses the personal data you provide to improve the user experience, facilitate representation and copresence among users, tailor your private results, and deliver these results to you. NONE of the personal data collected by the application will be shared with third parties under any circumstances. Derivative data includes information our servers automatically collect when you access the application, such as your actions that are integral to the application, including responses to questions, points wagered, points scored, questions submitted, and question votes. Importantly, none of your actions in the app, including your answers to questions, or 
voting behavior will be view-able by other users accessing the application or shared with third parties. Data about the electronic device(s) and browsers you use to play LezParlay may also be logged by our server. This includes your device model, manufacturer, operating system, and browser version. This information is collected to improve the user experience on different devices and to aid us in troubleshooting should you report an issue using LezParlay on your device. Aggregated and de-identified personal, derivative, device/browser and analytics data may be analyzed by LezParlay project personnel in order to better understand application usage, determine the popularity of certain content, and inform future versions of the game. All data analysis and reporting for these purposes will focus on highlighting group trends rather than individual responses.

\section{DATA SECURITY}

LezParlay is an html5 web app that features the most advanced technologies and internal protocols (layered security, https protocol, ssl certificates, tsl encryption in transit, etc.) to transmit, secure, and store and protect application data. These protocols safeguard privacy between communicating applications and their users on the internet. Also used to secure bank and credit card transactions online, these protocols ensure that no third party may eavesdrop or tamper with submitted data.

\section{POTENTIAL RISKS \& BENEFITS}

The risks associated with taking part in the competition lie in the potential for you to experience uncomfortable feelings due to the personal nature of some of the questions. Questions are somewhat edgy by design. They may ask, for example, how often you get drunk or have sex. You may choose to skip sensitive questions without penalty. However, we can only provide you points, scores, and detailed results corresponding to the questions you personally answer. You may also experience discomfort upon receipt of your detailed results. For example, it may be distressing to learn that other lesbians your age are drinking much less than you and exercising much more than you. You may opt out of receiving detailed feedback if you wish to do so. You may also choose to discontinue your participation in the competition at any time. This means if anything makes you feel too uncomfortable just quit playing! For some, participation in LezParlay may satisfy curiosities about LBQ communities or help to diminish harmful stereotypes. For others, learning about health and relationship behaviors of other lesbian and queer women may benefit their own health behaviors and relationships. Others may simply find entertainment in the wagers, scoring and results. De-identified, aggregated data collected by LezParlay may also help shape future programming by LezParlay community partners, which in turn, may benefit your future well-being.

\section{PRIZES}

The goal in LezParlay is to accumulate as many points as possible in each round and across rounds. You can earn points in each round by guessing and betting on the attitudes and behaviors of other LBQ women in your age-group and then reporting on your own attitudes \& behaviors. In each round: a variable cash prize ranging from $\$ 50$ to $\$ 500$ will be awarded to the player who scores the most points that round. In the event of ties, the top scorer who placed her bets earliest in the PARLAY period will be awarded the cash prize. After 6 rounds: Grand prizes 
will be awarded to the top 3 cumulative scorers in each age-group (20's, 30s, 40s, 50+) after all rounds have been played. In each age-group, first-place will receive $\$ 1000$, second place will receive $\$ 500$ and third place will receive $\$ 250$. In the event of ties, the cash prize will be awarded to the player who, on average across rounds played, placed her bets earliest.

\section{GROUNDS FOR DISQUALIFICATION}

LezParlay is designed for lesbian, bisexual and queer-identified women living in the US of A. In effort to keep the game completely cis male and Russian bot free, users may be disqualified from the competition and have their account deleted if they appear to clearly NOT be an LBQ woman or residing in the US. Other grounds for disqualification include creating multiple accounts linked to different mobile numbers (in order to submit bets more than once each round) or attempting to cheat by any other means. In the spirit of healthy competition, we ask that you keep the detailed results you receive in the game private. This means not sharing any of the results you receive on social media or in private communication with friends until all rounds of the competition have been played. You may be disqualified if you violate this rule!

\section{SUPPORT \& TROUBLESHOOTING}

If you experience trouble with the app on your mobile device or computer or are unable to login please email a description of your issue to support@lezparlay.com. Be sure to include the mobile phone number and email address associated with your LezParlay account so we can quickly fix any issues. We will shoot to resolve all issues within 24 hours.

\section{ADDITIONAL INFORMATION}

For additional information, questions about data usage, scoring, prizes, please contact Sarah Boyle, LezParlay's founder, at sarah@lezparlay.com or phone at 310.568.6681 
2. Official Tester Invite Pop-Up Screen (End of Round 3 for eligible players)

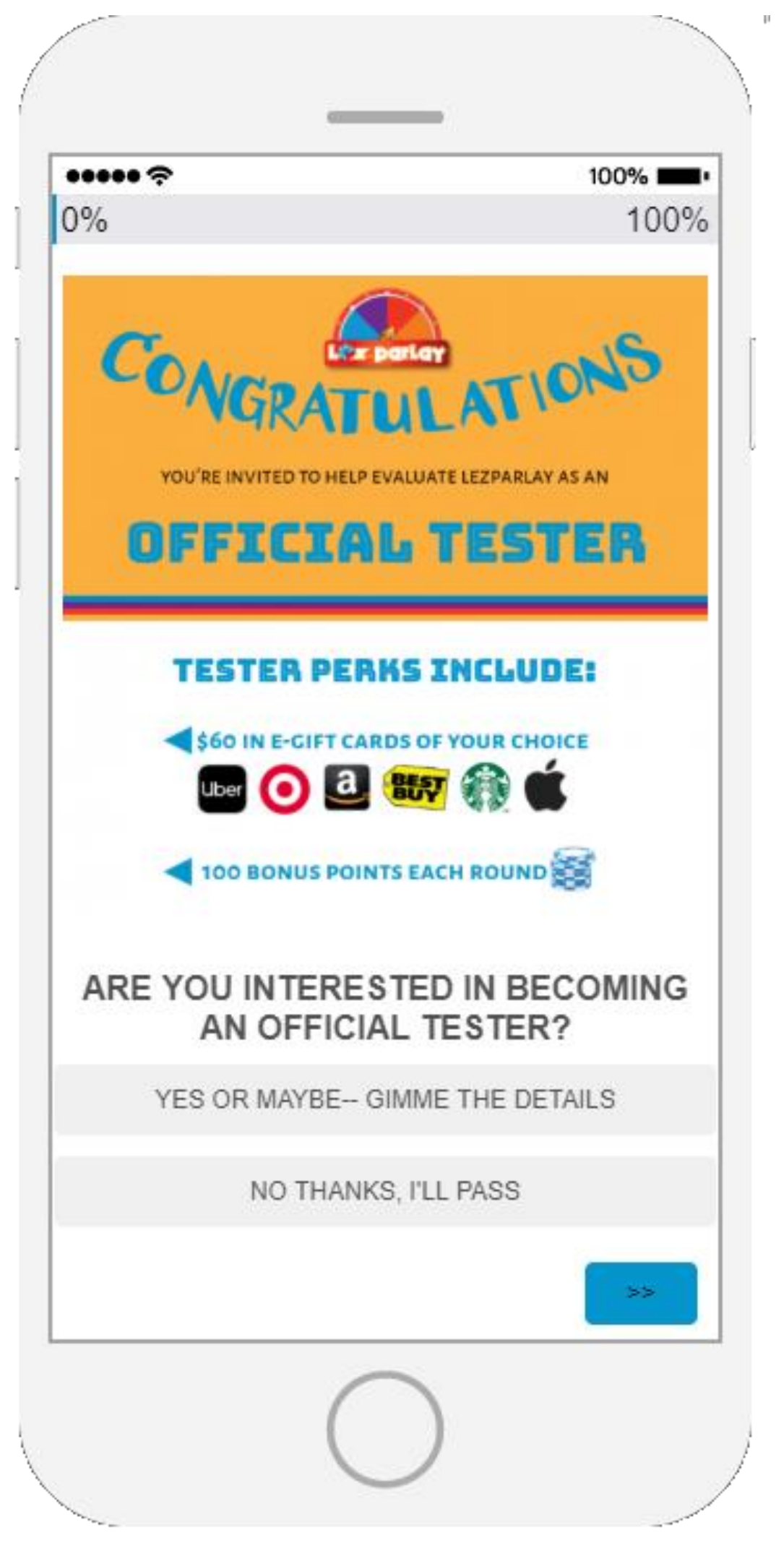


3. Evaluation Study Informed Consent Form

(End of Round 3 for eligible players interested in participating)

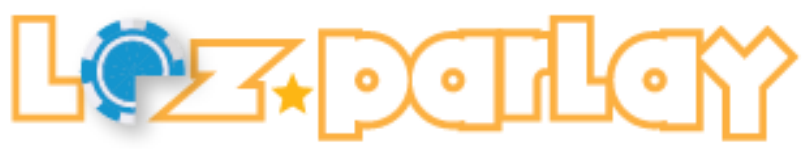

\section{EUALUATION STUDY INFO \& CONSENT}

\section{PURPOSE}

LezParlay is grant-funded and we hope to apply for additional funds to continue the game in the future, add new features, improve the question topics and detailed results, and make future versions as enjoyable and beneficial as possible for lesbian, bisexual, and queer women. As such, the goals of this study are to: 1) evaluate the potential benefits associated with question topics and detailed results received in the LezParlay competition; and, 2) gather feedback on the player experience and areas in which the competition and app might be improved.

\section{STUDY PARTICIPATION}

Based on your verified status and participation in initial rounds of LezParlay, you have an opportunity to take part in this evaluation study as an Official Tester. Participation in the evaluation study involves playing remaining rounds of LezParlay and then completing a brief feedback survey about your experience in the competition following the final round of play. Each round of LezParlay will take no longer than 7 minutes to complete and the brief feedback survey will take no longer than 12 minutes to complete.

\section{INCENTIVES}

Official testers who complete remaining rounds of the competition (Completing Rounds 2 through 7) and submit the brief feedback survey at the end of the game will receive a $\$ 60$ in e-gift card of their choice (e.g., iTunes, Amazon, Target, Starbucks) to compensate them for their time and valuable insight. Importantly, as an Official Tester taking part in the evaluation study, you remain eligible for the cash prizes to be awarded to the competition's top scorers.

\section{DATA USE}

As a participant in the evaluation study, your demographic data, feedback survey data and responses to questions in LezParlay rounds may be aggregated with the responses of other 
study participants and analyzed by the competition's founding psychologists at Loyola Marymount University. All research reports will de-identify all data from study participants and focus on highlighting group trends rather than individual responses.

\section{CONFIDENTIALITY}

While you may be visible and therefore potentially recognizable to other players in the game if you elect to upload a profile photo or bitmoji (as outlined in the app's Terms of Service and Privacy Policy), your participation in the Evaluation Study is completely confidential. There is no indication of which players are participating in the evaluation study and which are not. This means that no one will know you are participating in the evaluation study unless you tell them.

\section{DATA SECURITY}

As with all LezParlay data, feedback survey data is protected by Transport Layer Security (TLS) encryption (also known as HTTPS), which is a protocol that ensures privacy between communicating applications and their users on the internet. Also used to secure bank and credit card transactions online, TLS ensures that no third party may eavesdrop or tamper with submitted data.

\section{POTENTIAL RISKS \& BENEFITS}

There are no additional risks associated with participation in the evaluation study beyond the personal nature of some of the questions to appear in LezParlay rounds and detailed results delivered in the game which may be distressing (As outlined in the Terms of Service and Privacy Policy you previously accepted). Benefits associated with evaluation study participation include the opportunity to share what you liked about LezParlay, what you disliked, and any ideas you may have about making it better with the competition's founders. In this way, you can help shape future versions of the competition and ensure that future versions are as satisfying and beneficial for you as possible.

Participation in the evaluation study is voluntary and you are free to stop participating at any time. To discontinue study participation, either simply stop playing LezParlay or email us at eval@lezparlay.com and let us know of your decision if you wish to no longer participate in the evaluation study but still wish to take part in the competition.

\section{FOR MORE INFORMATION}

If you have any questions or concerns about your participation in the evaluation study please contact the study's director, Sarah Boyle, on the campus of Loyola Marymount University, office phone number (310) 568-8661, email address sarah.boyle@lmu.edu If you have questions regarding your rights as a research participant, you may contact Dr. David Moffet, Chair of the Human Subjects Review Committee at Loyola Marymount 
University, at (310) 338-4400 or david.moffet@Imu.edu. At the conclusion of this study, a summary of findings will be made publicly viewable at https://clinicaltrials.gov/ct2/show/NCT03884478

\section{PLEASE CHECK ONE OF THE BOXES BELOWH}

$\square \quad$ By checking this box, I acknowledge that:

- I have read and understood this informed consent information, and

- I would like to participate in the LezParlay evaluation study

By checking this box, I acknowledge that:

- I do not wish to participate in the LezParlay evaluation study. 


\section{Appendix C}

Email from Minor Dropped from Evaluation Study

(post-randomization but before the delivery of treatment PNF) 


\section{From: \\ Sent: S. \\ 2019 8:46 PM}

To: Boyle, Sarah

Subject: Lez parlay official testing

Hello Mrs. Boyle,

I was just emailing you about my participation, while I would love to do it I actually lied about my age just to check it out since I saw an ad for it on Instagram and knew prizes would be involved. My current age is 15 and if I were to participate as an official tester and play many rounds I fear that my different experiences would tamper with your data and I wouldn't want to skew it if you're planning on actually doing anything with it, which it appears you are. I would actually really enjoy to participate but seeing as the youngest age group available is 20 's I sincerely wouldn't want to interfere with your data. You don't have to email me back, but I would like to suggest a teen age group. You could probably get investors this way because teen data is always very valuable. With slightly different questions you could create a more palatable survey for younger adults, and if you did this I would be willing to get more kids my age involved with it to make sure you have enough people doing it to justify the age group. However I completely understand if it's not part of your vision for this game, and I don't know much about this kind of stuff because I'm only 15 so it would be completely fair to take everything I said with a grain of salt. I hope you have a good day though, keep up the good work! 


\section{Appendix D}

Data Cleaning \& Preliminary Analysis of Structural Stigma Indices 
Study participants took part in the LezParlay competition from 44 different states and 221 different counties. Structural stigma indices were derived from secondary data source variables through a two-step process that involved reverse-scoring (so that higher values were indicative of a harsher social climate for sexual minorities) and then z-scoring (so that all indices were on the same scale). Specific procedures for each structural stigma measure are detailed in Table D1.

Table D1. Structural stigma indices, data sources, and data cleaning procedures

\begin{tabular}{|c|c|c|c|}
\hline Level & Construct & Source \& References & Data Cleaning Procedures \\
\hline State & $\begin{array}{l}\text { Number of same-sex couples } \\
\text { per } 1000 \text { households }\end{array}$ & $\begin{array}{l}\text { Movement Advancement Project, } \\
2019\end{array}$ & $\begin{array}{l}\text { Subtracted from } 100 \text { to indicate the number of } \\
\text { heterosexual/opposite-sex couples per } 1000 \\
\text { households, then z-scored }\end{array}$ \\
\hline State & $\begin{array}{l}\text { Equality score based on tally } \\
\text { of LGBTQ supportive } \\
\text { policies/ legislature }\end{array}$ & $\begin{array}{l}\text { Movement Advancement Project, } \\
2019\end{array}$ & $\begin{array}{l}\text { Subtracted from the highest state equality score } \\
\text { to indicate less supportive policies/legislature, } \\
\text { then z-scored }\end{array}$ \\
\hline State & $\begin{array}{l}\text { Percent endorsing positive } \\
\text { opinions about gays and } \\
\text { lesbians }\end{array}$ & Lax \& Phillips, 2009 & $\begin{array}{l}\text { Percent of respondents endorsing positive } \\
\text { opinions subtracted from } 100 \text { so that higher } \\
\text { scores indicate negative public opinion, then z- } \\
\text { scored }\end{array}$ \\
\hline County & $\begin{array}{l}\text { Number of same-sex couples } \\
\text { per } 1000 \text { households }\end{array}$ & $\begin{array}{l}\text { Williams' Institute LGBT Data } \\
\text { Interactive, } 2019\end{array}$ & $\begin{array}{l}\text { Subtracted from } 100 \text { to indicate the number of } \\
\text { heterosexual/opposite-sex couples per } 1000 \\
\text { households, then z-scored }\end{array}$ \\
\hline County & $\begin{array}{l}2016 \text { U.S. Presidential } \\
\text { Election republican versus } \\
\text { democrat vote differential }\end{array}$ & McGovern, 2018 & $\begin{array}{l}\text { Percent who voted for Trump minus percent who } \\
\text { voted for Clinton so higher differentials indicate } \\
\text { greater conservativism, then } z \text {-scored. }\end{array}$ \\
\hline County & $\begin{array}{l}\text { Number of LGBTQ } \\
\text { Community Centers }\end{array}$ & $\begin{array}{l}\text { Centerlink Center Directory, } 2019 \\
\text { (combined with Google searches to } \\
\text { double-check each participant county) }\end{array}$ & $\begin{array}{l}\text { Subtracted from the max number of centers } \\
\text { found in a county so that higher scores indicate } \\
\text { fewer community centers, then } z \text {-scored }\end{array}$ \\
\hline
\end{tabular}

As shown below in Table D2, all six structural stigma indices were significantly correlated with one another (all $p$ s <.001), ranging from $r=.88$ on the high end to $r=.30$ on the low end. 
Table D2

Correlations between structural stigma indices $(N=499)$

\begin{tabular}{lcccccc}
\hline & 1 & 2 & 3 & 4 & 5 & 6 \\
\hline 1. Unsupportive legislature/policies in state & - & $.88^{* * *}$ & $.62^{* *}$ & $.53^{* * *}$ & $.35^{* * *}$ & $.34^{* *}$ \\
2. Negative attitudes toward gays in state & & - & $.59^{* * *}$ & $.53^{* * *}$ & $.30^{* * *}$ & $.32^{* * *}$ \\
3. Heterosexual population density in state & & & - & $.44^{* *}$ & $.30^{* * *}$ & $.48^{* * *}$ \\
4. Conservative voting differential in county & & & & - & $.61^{* * *}$ & $.67^{* * *}$ \\
5. Lack of LGBTQ resources in county & & & & & - & $.43^{* *}$ \\
6. Heterosexual population density in county & & & & & & - \\
\hline
\end{tabular}

\section{Exploratory Factor Analysis}

As previous structural stigma work has focused exclusively structural stigma indices at the state-level, an exploratory factor analysis was conducted to examine the factor structure of the six state and county-level structural stigma measures and determine how indices should be combined for analysis. Principal axis factoring (PAF) with the direct Oblimin (oblique) rotation method was used in order to allow extracted factors to be correlated. The Kaiser rule (eigenvalues greater than 1) was used to determine the number of factors to be extracted.

Communalities and factor loadings from the pattern matrix were inspected using a cut-off of.40 to identify poorly fitting measures that should be considered for removal.

Initial results supported a two-factor solution, $\mathrm{KMO}=.96, \chi 2(499)=1776.01, p<.001$, with the two factors accounting for $76.40 \%$ of variance across items. The three state-level items loaded on one factor (all loadings above.51) and the three county-level items loaded on the other factor (all loadings above.62). As shown below, the only measure that substantially loaded on both factors was the state-level item assessing population density heterosexual couples, which loaded more than twice as well on factor $1(.52)$ than factor $2(.23)$. As expected, the two extracted factors were significantly correlated, $r=.58$. 
Table D2

Structural Stigma Exploratory Factor Analysis Results

\begin{tabular}{lccc}
\hline & Communalities & $\begin{array}{c}\text { Factor 1 } \\
\text { (state) }\end{array}$ & $\begin{array}{c}\text { Factor 2 } \\
\text { (county) }\end{array}$ \\
\hline Measure & & & \\
Unsupportive legislature and policies in state & .93 &. $\mathbf{9 9}$ & \\
Negative public opinion toward gays in state & .84 & .94 & \\
Heterosexual couple population density in state & .43 & .51 & .23 \\
Conservative voting differential in county & .84 & & $\mathbf{. 8 7}$ \\
Heterosexual population density in county & .59 & & $\mathbf{. 7 7}$ \\
Lack of LGBTQ-specific resources in county & .42 & & $\mathbf{. 6 2}$ \\
\hline
\end{tabular}

Internal consistencies were high among the three state-level measures (alpha=.87), three county-level measures (alpha $=.80$ ) and across all six measures (alpha $=.86$ ). Based on factor loadings, state and county specific 3 item measures of structural stigma were computed. Tests of moderation separately examined respective measures of structural stigma at the state and countylevel as potential moderators of intervention efficacy (without controlling for the other measure). Due to the strong correlation between state and county-level measures $(r=.58)$ however, efficacy main effects analyses controlled for a total structural stigma score which was derived by summing across state and county specific indices. This was done to reduce multi-collinearity in main effect GLMM models. 
Appendix E

Supplemental Results and Model Information 


\section{Table E1}

For demonstration purposes, $\mathrm{H} 1$ and $\mathrm{H} 2$ tested via single step linear regression models with dummy variables representing all categorical variables (rather than GLMM; N=400).

\begin{tabular}{|c|c|c|c|c|c|c|}
\hline Test & Predictor & $B$ & $S E$ & $\mathrm{t}$ & $F \Delta$ & $R^{2} \Delta$ \\
\hline & Queer sexual identity & -.03 & .07 & -.41 & & \\
\hline & Bisexual sexual identity & .12 & .06 & 1.91 & & \\
\hline & Racial/ethnic minority status & -.01 & .05 & -.12 & & \\
\hline & Age in years & .0001 & .004 & .04 & & \\
\hline & Single relationship status & .05 & .05 & .86 & & \\
\hline & Total interpersonal stigma exposure & -.03 & .02 & -1.13 & & \\
\hline & Total structural stigma & .01 & .02 & .33 & & \\
\hline & Z-Alcohol use T1 & $.75^{* * * *}$ & .04 & 20.67 & & \\
\hline $\mathrm{H} 1 \mathrm{~b}$ & Alcohol+Coping PNF & $-.54 * * *$ & .06 & -8.47 & & \\
\hline \multirow[t]{12}{*}{ H1a } & Alcohol PNF & $-.51 * * *$ & .06 & -8.44 & & \\
\hline & Control PNF (ref group) & & & & & \\
\hline & Model Summary & & & & $60.03 * * *$ & .60 \\
\hline & Queer sexual identity & -.03 & .07 & -.41 & & \\
\hline & Bisexual sexual identity & .12 & .06 & 1.91 & & \\
\hline & Racial/ethnic minority status & -.01 & .05 & -.12 & & \\
\hline & Age in years & .0001 & .004 & .04 & & \\
\hline & Single relationship status & .05 & .05 & .86 & & \\
\hline & Total interpersonal stigma exposure & -.03 & .02 & -1.13 & & \\
\hline & Total structural stigma & .01 & .02 & .33 & & \\
\hline & Z-Alcohol use T1 & $.75 * * *$ & .04 & 20.67 & & \\
\hline & Control PNF & $-.54 * * *$ & .06 & -8.47 & & \\
\hline \multirow[t]{3}{*}{$\mathrm{H} 2$} & Alcohol+Coping PNF & -.03 & .06 & .56 & & \\
\hline & Alcohol PNF (ref group) & & & & & \\
\hline & Model Summary & & & & $60.03 * * *$ & $.60 * * *$ \\
\hline
\end{tabular}

$p<.001$ 
Table E2

Full CC PROCESS models testing condition*interpersonal stigma interactions on alcohol use at followup.

\begin{tabular}{|c|c|c|c|c|c|c|c|}
\hline Model & Predictor & $B$ & $S E$ & $T$ & $F$ & $d f$ & $R^{2}$ \\
\hline \multirow[t]{14}{*}{6} & Queer sexual identity & -.03 & .07 & -.42 & & & \\
\hline & Bisexual sexual identity & .12 & .06 & 1.84 & & & \\
\hline & Racial/ethnic minority & -.02 & .05 & -.33 & & & \\
\hline & Age in years & .001 & .003 & -.21 & & & \\
\hline & Single relationship status & .06 & .06 & 1.01 & & & \\
\hline & Total structural stigma & -.001 & .03 & -.004 & & & \\
\hline & z-Alcohol use composite T1 & $.75 * * *$ & .04 & $21.08 * * *$ & & & \\
\hline & Interpersonal Stigma Exposure T1 & .05 & .08 & .62 & & & \\
\hline & Alcohol+Coping PNF & $-.45^{* * *}$ & .08 & $-5.32 * * *$ & & & \\
\hline & Alcohol PNF & $-.42 * * *$ & .08 & $-5.24 * * *$ & & & \\
\hline & Alcohol+Coping PNF*T1 Int stigma & -.15 & .09 & -1.60 & & & \\
\hline & Alcohol PNF* T1 Int stigma & -.14 & .10 & -1.41 & & & \\
\hline & Model Summary & & & & $66.07 * * *$ & 12,387 & $.61 * * *$ \\
\hline & Contribution of Interaction Terms & & & & 1.41 & 2,387 & .003 \\
\hline \multirow[t]{14}{*}{7} & Queer sexual identity & -.03 & .06 & -.36 & & & \\
\hline & Bisexual sexual identity & .10 & .06 & 1.56 & & & \\
\hline & Racial/ethnic minority & -.03 & .05 & -.54 & & & \\
\hline & Age in years & .001 & .003 & -.21 & & & \\
\hline & Single relationship status & .06 & .06 & 1.14 & & & \\
\hline & Total structural stigma & -.003 & .06 & -.12 & & & \\
\hline & z-Alcohol use composite T1 & $.75^{* * *}$ & .03 & $21.78 * * *$ & & & \\
\hline & Interpersonal Stigma Exposure T2 & $.23 * * *$ & .06 & $3.66^{* * *}$ & & & \\
\hline & Alcohol+Coping $\mathrm{PNF}^{\mathrm{A}}$ & $-.30 * * *$ & .09 & $-3.51 * * *$ & & & \\
\hline & Alcohol PNF ${ }^{\mathrm{B}}$ & $-.25 * *$ & .08 & $-2.96 * *$ & & & \\
\hline & Alcohol+Coping PNF*T2 Int stigma & $-.42 * * *$ & .08 & $-5.18 * * *$ & & & \\
\hline & Alcohol PNF* T2 Int stigma &.$- .38 * * *$ & .09 & $-4.63 * * *$ & & & \\
\hline & Model Summary & & & & $72.78 * * *$ & 12,387 & $.64 * * *$ \\
\hline & Contribution of Interaction Terms & & & & $15.57 * * *$ & 2,387 & .03 \\
\hline
\end{tabular}

${ }^{*} p<.05 ; * * p<.01 ; * * * p<.001$ 
Table E3

Full CC PROCESS models testing condition* structural stigma interactions on alcohol use at follow-up ( $N=400 ;$ continued on next page)

\begin{tabular}{|c|c|c|c|c|c|c|c|}
\hline Model & Predictor & $B$ & $S E$ & $T$ & $F$ & $d f$ & $R^{2}$ \\
\hline \multirow[t]{14}{*}{8} & Queer sexual identity & -.03 & .07 & -.50 & & & \\
\hline & Bisexual sexual identity & .11 & .06 & 1.82 & & & \\
\hline & Racial/ethnic minority & -.01 & .06 & -.19 & & & \\
\hline & Age in years & .001 & .003 & .009 & & & \\
\hline & Single relationship status & .05 & .06 & .87 & & & \\
\hline & Total interpersonal stigma & -.03 & .03 & -1.08 & & & \\
\hline & z-Alcohol use composite $\mathrm{T} 1$ & $.75 * * *$ & .04 & $20.41 * * *$ & & & \\
\hline & Total structural stigma & .02 & .05 & .46 & & & \\
\hline & Alcohol+Coping PNF & $-.54 * * *$ & .06 & $-8.65 * * *$ & & & \\
\hline & Alcohol PNF & $-.51 * * *$ & .06 & $-7.98 * * *$ & & & \\
\hline & Alcohol+Coping PNF*Total Str Stigma & -.07 & .08 & -.89 & & & \\
\hline & Alcohol PNF*Total Str Stigma & -.01 & .07 & -.18 & & & \\
\hline & Model Summary & & & & $66.01 * * *$ & 12,387 & $.61 * * *$ \\
\hline & Contribution of Interaction Terms & & & & .45 & 2,387 & .001 \\
\hline \multirow[t]{14}{*}{9} & Queer sexual identity & -.04 & .07 & -.54 & & & \\
\hline & Bisexual sexual identity & .12 & .07 & 1.80 & & & \\
\hline & Racial/ethnic minority & -.01 & .05 & -.19 & & & \\
\hline & Age in years & .001 & .003 & -.009 & & & \\
\hline & Single relationship status & .05 & .06 & .91 & & & \\
\hline & Total interpersonal stigma & -.03 & .03 & -1.05 & & & \\
\hline & z-Alcohol use composite T1 & $.75 * * *$ & .04 & $20.51 * * *$ & & & \\
\hline & County-level structural stigma & .01 & .05 & .26 & & & \\
\hline & Alcohol+Coping PNF & $-.54 * * *$ & .06 & $-8.63 * * *$ & & & \\
\hline & Alcohol PNF & $-.51 * * *$ & .06 & $-7.98 * * *$ & & & \\
\hline & Alcohol+Coping PNF*County Str Stigma & -.08 & .07 & -1.10 & & & \\
\hline & Alcohol PNF*County Str Stigma & -.001 & .07 & .35 & & & \\
\hline & Model Summary & & & & $65.27 * * *$ & 12,387 & $.61^{* * *}$ \\
\hline & Contribution of Interaction Terms & & & & .60 & 2,387 & .001 \\
\hline
\end{tabular}




\begin{tabular}{|c|c|c|c|c|c|c|c|}
\hline Model & Predictor & $B$ & $S E$ & $T$ & $F$ & $d f$ & $R^{2}$ \\
\hline \multirow[t]{14}{*}{10} & Queer sexual identity & -.03 & .07 & -.41 & & & \\
\hline & Bisexual sexual identity & .11 & .06 & 1.74 & & & \\
\hline & Racial/ethnic minority & -.009 & .05 & -.16 & & & \\
\hline & Age in years & .001 & .003 & .06 & & & \\
\hline & Single relationship status & .05 & .06 & .83 & & & \\
\hline & State-level structural stigma & .05 & .05 & 1.04 & & & \\
\hline & z-Alcohol use composite T1 & $.75 * * *$ & .04 & $20.64 * * *$ & & & \\
\hline & Interpersonal Stigma Exposure T2 & -.03 & .03 & -1.14 & & & \\
\hline & Alcohol+Coping PNF & $-.54 * * *$ & .06 & $-8.75 * * *$ & & & \\
\hline & Alcohol PNF & $-.51 * * *$ & .06 & $-8.02 * * *$ & & & \\
\hline & Alcohol+Coping PNF*State Str Stigma & -.06 & .07 & -.84 & & & \\
\hline & Alcohol PNF*State Str Stigma & -.03 & .06 & -.57 & & & \\
\hline & Model Summary & & & & $66.91 * * *$ & 12,387 & $.61 * * *$ \\
\hline & Contribution of Interaction Terms & & & & .35 & 2,387 & $<.001$ \\
\hline
\end{tabular}

${ }^{*} p<.05 ; * * p<.01 ; * * * p<.001$ 
Table E4

Full CC PROCESS models testing baseline drinking and demographics as moderators of the relationship between study condition and drinking at follow-up (continued on next page).

\begin{tabular}{|c|c|c|c|c|c|c|c|}
\hline Model & Predictor & $B$ & $S E$ & $t$ & $F$ & $d f$ & $R^{2}$ \\
\hline \multirow[t]{14}{*}{11} & Queer sexual identity & -.03 & .06 & -.46 & & & \\
\hline & Bisexual sexual identity & .11 & .06 & 1.69 & & & \\
\hline & Racial/ethnic minority & -.008 & .05 & -.15 & & & \\
\hline & Age in years & -.002 & .003 & -.07 & & & \\
\hline & Single relationship status & .02 & .05 & .32 & & & \\
\hline & Total interpersonal stigma & -.02 & .03 & -.88 & & & \\
\hline & Total structural stigma & .008 & .03 & .26 & & & \\
\hline & z-Alcohol use composite T1 & $.84 * * *$ & .07 & $11.80 * * *$ & & & \\
\hline & Alcohol+Coping PNF & $-.54 * * *$ & .06 & $-8.80 * * *$ & & & \\
\hline & Alcohol PNF & $-.51 * * *$ & .06 & $-8.09 * * *$ & & & \\
\hline & Alcohol+Coping PNF*T1 Alcohol Use & $-.25 * *$ & .09 & $-2.72 * *$ & & & \\
\hline & Alcohol PNF*T1 Alcohol Use & -.06 & .08 & -.82 & & & \\
\hline & Model Summary & & & & $66.41 * * *$ & 12,387 & $.62 * * *$ \\
\hline & Contribution of Interaction Terms & & & & $4.23 * *$ & 2,387 & $.01 * *$ \\
\hline \multirow[t]{14}{*}{12} & Queer sexual identity & -.03 & .07 & -.46 & & & \\
\hline & Bisexual sexual identity & .12 & .06 & 1.89 & & & \\
\hline & Racial/ethnic minority & -.01 & .05 & -.12 & & & \\
\hline & Age in years & .001 & .003 & .06 & & & \\
\hline & Total interpersonal stigma & -.03 & .02 & -1.06 & & & \\
\hline & Total structural stigma & .004 & .03 & .15 & & & \\
\hline & Single relationship status & .05 & .10 & .51 & & & \\
\hline & z-Alcohol use composite T1 & $.75^{* * * *}$ & .04 & $20.01 * * *$ & & & \\
\hline & Alcohol+Coping PNF & $-.52 * * *$ & .09 & $-6.23 * * *$ & & & \\
\hline & Alcohol PNF & $-.51 * * *$ & .08 & $-5.92 * * *$ & & & \\
\hline & Alcohol+Coping PNF*Single & -.04 & .13 & -.31 & & & \\
\hline & Alcohol PNF*Single & .02 & .13 & .16 & & & \\
\hline & Model Summary & & & & $65.63 * * *$ & 12,387 & $.61 * * *$ \\
\hline & Contribution of Interaction Terms & & & & .13 & 2,387 & $<.001$ \\
\hline
\end{tabular}




\begin{tabular}{|c|c|c|c|c|c|c|c|}
\hline Model & Predictor & $B$ & $S E$ & $T$ & $F$ & $d f$ & $R^{2}$ \\
\hline \multirow[t]{16}{*}{13} & Racial/ethnic minority & -.005 & .05 & -.88 & & & \\
\hline & Age in years & -.002 & .003 & -.06 & & & \\
\hline & Single relationship status & .04 & .06 & .69 & & & \\
\hline & Total interpersonal stigma & -.03 & .02 & -1.07 & & & \\
\hline & Total structural stigma & .001 & .03 & .02 & & & \\
\hline & z-Alcohol use composite T1 & $.76^{* * *}$ & .04 & $20.30 * * *$ & & & \\
\hline & Queer sexual identity & .04 & .12 & .32 & & & \\
\hline & Bisexual sexual identity & .14 & .12 & 1.19 & & & \\
\hline & Alcohol+Coping PNF & $-.52 * * *$ & .08 & $-5.64 * * *$ & & & \\
\hline & Alcohol PNF & $-.47 * * *$ & .08 & $-6.14 * * *$ & & & \\
\hline & Alcohol+Coping PNF*Queer identity & -.14 & .15 & -.89 & & & \\
\hline & Alcohol PNF*Queer identity & -.08 & .17 & -.44 & & & \\
\hline & Alcohol+Coping PNF*Bisex identity & .05 & .16 & .28 & & & \\
\hline & Alcohol PNF*Bisexual identity & -.09 & .15 & -.58 & & & \\
\hline & Model Summary & & & & $57.08 * * *$ & 14,385 & $.61 * * *$ \\
\hline & Contribution of Interaction Terms & & & & .48 & 4,385 & .002 \\
\hline \multirow[t]{14}{*}{14} & Queer sexual identity & -.03 & .07 & -.45 & & & \\
\hline & Bisexual sexual identity & .12 & .06 & 1.85 & & & \\
\hline & Racial/ethnic minority & -.002 & .10 & -.02 & & & \\
\hline & Age in years & .001 & .002 & .04 & & & \\
\hline & Total interpersonal stigma & -.03 & .03 & -1.05 & & & \\
\hline & Total structural stigma & .003 & .03 & .10 & & & \\
\hline & Single relationship status & .05 & .10 & .51 & & & \\
\hline & z-Alcohol use composite T1 & $.75^{* * *}$ & .04 & $20.34 * * *$ & & & \\
\hline & Alcohol+Coping PNF & $-.54 * * *$ & .10 & $-5.47 * * *$ & & & \\
\hline & Alcohol PNF & $-.50 * * *$ & .10 & $-5.23 * * *$ & & & \\
\hline & Alcohol+Coping PNF*Rac/Ethn Minor & -.01 & .13 & -.08 & & & \\
\hline & Alcohol PNF*Rac/Ethn Minority & -.004 & .13 & -.03 & & & \\
\hline & Model Summary & & & & $65.99 * * *$ & 12,387 & $.61 * * *$ \\
\hline & Contribution of Interaction Terms & & & & .003 & 2,387 & $<.001$ \\
\hline
\end{tabular}




\begin{tabular}{|c|c|c|c|c|c|c|c|}
\hline Model & Predictor & $B$ & $S E$ & $T$ & $F$ & $d f$ & $R^{2}$ \\
\hline \multirow[t]{14}{*}{15} & Queer sexual identity & -.03 & .07 & -.42 & & & \\
\hline & Bisexual sexual identity & .12 & .06 & 1.90 & & & \\
\hline & Racial/ethnic minority & -.008 & .05 & -.15 & & & \\
\hline & Single relationship status & .04 & .05 & .80 & & & \\
\hline & Total interpersonal stigma & -.03 & -.03 & -1.01 & & & \\
\hline & Total structural stigma & .004 & .004 & .13 & & & \\
\hline & z-Alcohol use composite $\mathrm{T} 1$ & $.75^{* * *}$ & .04 & $20.44 * * *$ & & & \\
\hline & Age in years & -.003 & .006 & -.48 & & & \\
\hline & Alcohol+Coping PNF & $-.58 * *$ & .25 & $-3.01 * *$ & & & \\
\hline & Alcohol PNF & $-.80^{*}$ & .27 & $-2.32 *$ & & & \\
\hline & Alcohol+Coping PNF*Age & .01 & .01 & 1.03 & & & \\
\hline & Alcohol PNF*Age & .004 & .01 & .32 & & & \\
\hline & Model Summary & & & & $65.27 * * *$ & 12,387 & $.61^{* * *}$ \\
\hline & Contribution of Interaction Terms & & & & .59 & 2,387 & .001 \\
\hline
\end{tabular}

${ }^{*} p<.05 ; * * p<.01 ; * * * p<.001$ 
Table E6

Summary of ITT main effect model results testing $H 1$ and $H 2(N=499)$

\begin{tabular}{|c|c|c|c|c|c|c|}
\hline Outcome & $B$ & $S E$ & Wald & $R R$ & $\begin{array}{c}95 \% \mathrm{CI} \\
{[R R]} \\
\end{array}$ & $\begin{array}{c}\text { Marginal } M(S E) \\
\text { Comparisons }\end{array}$ \\
\hline \multicolumn{7}{|l|}{ Model 1: T2 Alcohol Use Composite } \\
\hline Alcohol+Coping PNF ${ }^{\mathrm{a}}$ & $-.44 * * *$ & .06 & 64.04 & $.64 * * *$ & $.58-.72$ & $-.17(.04) * * * c$ \\
\hline Alcohol PNF ${ }^{b}$ & $-.41 * * *$ & .05 & 55.41 & $.66^{* * *}$ & $.59-.73$ & $-.14(.04) * * * c$ \\
\hline Control PNF (ref group) ${ }^{\mathrm{c}}$ & & & & & & $.28(.04)$ \\
\hline \multicolumn{7}{|l|}{ Model 2: T2 Estimated drinks per week ${ }^{l}$} \\
\hline Alcohol+Coping PNF ${ }^{\mathrm{a}}$ & $-.30 * * *$ & .05 & 28.98 & $.74 * * *$ & $.67-.83$ & $6.31(.25)^{* * * c}$ \\
\hline Alcohol PNF & $-.34 * * *$ & .06 & 36.41 & $.71 * * *$ & $.64-.80$ & $6.05(.26)^{* * * c}$ \\
\hline Control PNF (ref group) ${ }^{\mathrm{c}}$ & & & & & & $8.47(.33)$ \\
\hline \multicolumn{7}{|l|}{ Model 3: T2 Peak drinks ${ }^{2}$} \\
\hline Alcohol+Coping PNF & $-.23 * * *$ & .03 & 46.67 & $.80 * * *$ & $.74-.85$ & $4.36(.11)^{* * * c}$ \\
\hline Alcohol PNF ${ }^{b}$ & $-.24 * * *$ & .03 & 48.65 & $.79 * * *$ & $.73-.84$ & $4.32(.12) * * * c$ \\
\hline Control PNF (ref group) ${ }^{\mathrm{c}}$ & & & & & & $5.49(.13)$ \\
\hline \multicolumn{7}{|l|}{ Model 4: T2 Negative Consequences ${ }^{3}$} \\
\hline Alcohol+Coping PNF ${ }^{\mathrm{a}}$ & $-.24 * * *$ & .05 & 24.38 & $.78 * * *$ & $.71-.86$ & $2.36(.10)^{* * * c}$ \\
\hline Alcohol PNF ${ }^{b}$ & $-.26 * * *$ & .05 & 29.29 & $.77 * * *$ & $.71-.85$ & $2.33(.09)^{* * * c}$ \\
\hline Control PNF (ref group) ${ }^{\mathrm{c}}$ & & & & & & $3.01(.10)$ \\
\hline
\end{tabular}

$* * * p<.001$ 


\section{Table E7}

Summary of ITT moderation model results testing H3a and H5b $(N=499)$

\begin{tabular}{cccccc}
\hline Predictor & $B$ & $S E$ & $t$ & $F \Delta$ & $R^{2} \Delta$ \\
\hline Model 12: T2 Interpersonal Stigma as moderator & & & & $12.92^{* * *}$ & .02 \\
Alcohol+Coping PNF*T1 Alcohol Consumption & $-.32^{* * *}$ & .08 & $-4.02 * * *$ & & \\
Alcohol PNF*T1 Alcohol Consumption & $-.34 * * *$ & .08 & $-4.63 * * *$ & & \\
Model 12: Baseline Drinking as moderator & & & & $2.73^{*}$ & .005 \\
Alcohol+Coping PNF*T1 Alcohol Consumption & $-.17 *$ & .08 & $-2.21^{*}$ & & \\
Alcohol PNF*T1 Alcohol Consumption & -.02 & .06 & -.72 & & \\
\hline
\end{tabular}

$* p<.05 ; * * * p<.001$ 


\section{Appendix F}

Applicable Feedback Survey Sections \& Study Debriefing Statement 
\$\{e://Field/USERNAME\}, thanks for helping us evaluate

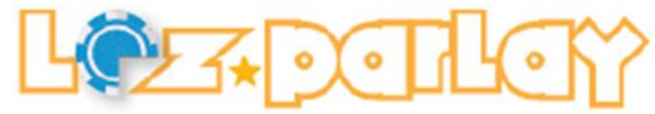

This survey will take about 10 minutes to complete and we'll send you a \$20 giftcard (within 24 hours) to thank you for your time. First, tell us about yourself so we know who the feedback is coming from....

What is your current age in years?

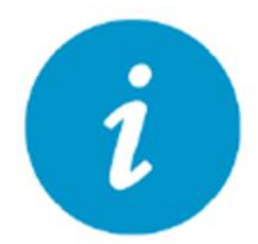

Which best describes your sexual identity?

lesbian

bisexual

$\bigcirc$ queer

For how many years has this been your sexual identity?

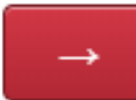




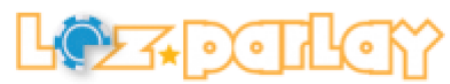

Great, thanks!

Next, let us know how much you DISLIKED or LIKED each aspect of LezParlay...
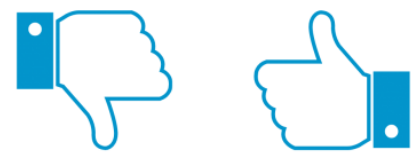

Disliked very much
Disliked
Neutral
Liked very

much

The "stereotype challenge" concept

The topics \& questions

The detailed results you received

The ability to browse player profiles

The ability to submit \& vote on questions

The ability to bet on your guesses being correct in some rounds

Text messages from LezParlay

Emails from LezParlay

The leaderboards showing the Top 100 Scorers each round

The ability to win money \& get gift cards

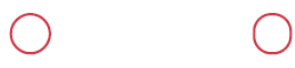

0

O

O

O

O

O

0

O

O

0
0

O

0

0

0

$O$

$O$

0

0

P

0

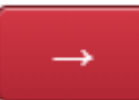




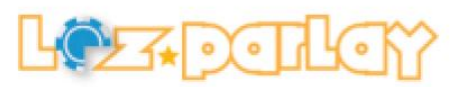

Did you find taking part in the competition to be beneficial to you at all?

No

Yes

$\bigcirc$

[IF YES]

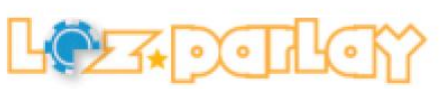

Great! Please use the text box below to briefly describe how the competition was beneficial for you

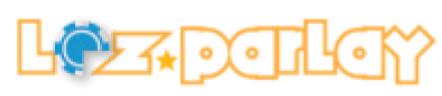

Do you any other ideas about how LezParlay might be improved?

What would you like to see be different in the next version? 


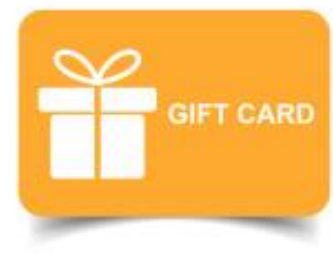

$\$\{\mathrm{e}: / /$ Field/USERNAME\}, the preferred email address we have for you is:

$\$\{\mathrm{~m}: / /$ ExternalDataReference $\}$

If you'd like your final gift card to be sent to an email address other than this please enter it below.

(Otherwise just click next for a final message from the LezParlay team)

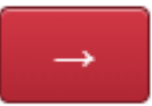

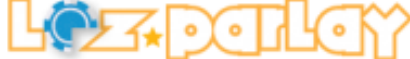 \\ A FINAL THANKS \& DISCLOSURE...}

Thanks again for helping us evaluate LezParlay! Before you go we wanted to give you some more information about the detailed results you received in the LezParlay as an Official Tester. In addition to getting your feedback and ideas for the next version of the competition, another goal of this study was to evaluate whether delivering results for health-related topics in LezParlay had any benefits for players' health and well-being. To fully examine this, we had to make sure that balanced groups of Official Testers received more and less health-related feedback.

So, full disclosure, the topics you received detailed results on in Rounds 3 and 4 of the game were not totally random in the same way they were in all of the other rounds (where they were determined by the doors you chose to open). Instead, as an Official Tester you were randomized to receive a specific sequence of feedback in Rounds 3 and 4 and the doors you selected were fixed to open to either health-related topics or topics unrelated to health. So, we're not sure if the health-related detailed results were beneficial yet, but analyzing the data from this study will allow us to find out! We also plan to examine whether you all reported different types of psychological benefits and/or differential levels

liking for aspects of the competition based on the sequences of detailed results you received.

If you are interested in finding out what we learn here please let us know by sending an email to sarah@lezparlay.com and we'll be sure to send you a summary of results when they are ready. Thanks again for your participation! 


\section{Bibliography}

Allam, A., Kostova, Z., Nakamoto, K., \& Schulz, P. J. (2015). The effect of social support features and gamification on a Web-based intervention for rheumatoid arthritis patients: randomized controlled trial. Journal of Medical Internet Research, 17, e14. https://doi.org/10.2196/jmir.3510.

Allen, J. L., \& Mowbray, O. (2016). Sexual orientation, treatment utilization, and barriers for alcohol related problems: Findings from a nationally representative sample. Drug and Alcohol Dependence, 161, 323-330. https://doi.org/10.1016/j.drugalcdep.2016.02.025

Atkins, D. C., Baldwin, S. A., Zheng, C., Gallop, R. J., \& Neighbors, C. (2013). A tutorial on count regression and zero-altered count models for longitudinal substance use data. Psychology of Addictive Behaviors, 27, 166-177. https://doi.org/10.1037/a0029508

Austin, E. L., \& Irwin, J. A. (2010). Health behaviors and health care utilization of southern lesbians. Women's Health Issues, 20, 178-184. https://doi.org/10.15288/jsad.2010.71.295

Babor, T. F., McRee, B. G., Kassebaum, P. A., Grimaldi, P. L., Ahmed, K., \& Bray, J. (2007). Screening, Brief Intervention, and Referral to Treatment (SBIRT) toward a public health approach to the management of substance abuse. Substance Abuse, 28, 7-30. https://doi.org/10.1300/J465v28n03_03

Baer, J. S. (1993). Etiology and secondary prevention of alcohol problems with young adults. In Addictive behaviors across the life span: Prevention, treatment, and policy issues (pp. 111-137). Thousand Oaks, CA, US: Sage Publications, Inc.

Bandermann, K. M., \& Szymanski, D. M. (2014). Exploring coping mediators between heterosexist oppression and posttraumatic stress symptoms among lesbian, gay, and 
bisexual persons. Psychology of Sexual Orientation and Gender Diversity, 1, 213-224. https://doi.org/10.1037/sgd0000044

Baptista, G., \& Oliveira, T. (2019). Gamification and serious games: A literature meta-analysis and integrative model. Computers in Human Behavior, 92, 306-315. https://doi.org/10.1016/j.chb.2018.11.030

Bauer, G. R., \& Wayne, L. D. (2005). Cultural sensitivity and research involving sexual minorities. Perspectives on Sexual and Reproductive Health, 37, 45-47.

Bedendo, A., Ferri, C. P., de Souza, A. A. L., Andrade, A. L. M., \& Noto, A. R. (2019a). Pragmatic randomized controlled trial of a web-based intervention for alcohol use among Brazilian college students: Motivation as a moderating effect. Drug and Alcohol Dependence, 199, 92-100. https://doi.org/10.1016/j.drugalcdep.2019.02.021

Bedendo, A., McCambridge, J., Gaume, J., Souza, A. A. L., Souza-Formigoni, M. L. O., \& Noto, A. R. (2019b). Components evaluation of a web-based personalised normative feedback intervention for alcohol use among college students: a pragmatic randomised controlled trial with a dismantling design. Addiction, (ahead of print). https://doi.org/10.1111/add.14923

Berkowitz, A. D. (2004). The Social Norms Approach: Theory, Research, and Annotated Bibliography. https://vcut.org/buh4dw3ssoi.pdf

Bertholet, N., Daeppen, J. B., Cunningham, J. A., Burnand, B., Gmel, G., \& Gaume, J. (2016). Are young men who overestimate drinking by others more likely to respond to an electronic normative feedback brief intervention for unhealthy alcohol use? Addictive Behaviors, 63, 97-101. https://doi.org/10.1016/j.addbeh.2016.07.015. 
Blanton, H., \& Christie, C. (2003). Deviance regulation: A theory of action and identity. Review of General Psychology, 7, 115-149. https://doi.org/10.1037\%2F1089-2680.7.2.115

Blok, A. C., Sadasivam, R. S., Amante, D. J., Kamberi, A., Flahive, J., Morley, J.,... \& Houston, T. K. (2019). Gamification to motivate the unmotivated smoker: the "take a break" digital health intervention. Games for Health Journal, 8, 275-284. https://doi.org/10.1089/g4h.2018.0076

Blume, A. W. (2016). Advances in substance abuse prevention and treatment interventions among racial, ethnic, and sexual minority populations. Alcohol Research : Current Reviews, 38, 47-54.

Boendermaker, W. J., Maceiras, S. S., Boffo, M., \& Wiers, R. W. (2016). Attentional bias modification with serious game elements: evaluating the shots game. JMIR Serious Games, 4, e20. https://doi.org/10.2196\%2Fgames.6464

Borsari, B., \& Carey, K. B. (2003). Descriptive and injunctive norms in college drinking: A meta-analytic integration. Journal of Studies on Alcohol and Drugs, 64, 331-341. doi: 10.15288/jsa.2003.64.331.

Boyle, E., Connolly, T. M., \& Hainey, T. (2011). The role of psychology in understanding the impact of computer games. Entertainment Computing, 2, 69-74. https://doi.org/10.1016/j.entcom.2010.12.002

Boyle, S. C., Costine, L. D., \& LaBrie, J. W. (2017). Cross-sectional and feed-forward relationships between peer coping norms and LGB adults' own coping-motivated alcohol use following stressors. Alcoholism, Clinical and Experimental Research, 41, 259A.

Boyle, S. C., Earle, A. M., LaBrie, J. W., \& Smith, D. J. (2017). PNF 2.0? Initial evidence that gamification can increase the efficacy of brief, web-based personalized normative 
feedback alcohol interventions. Addictive Behaviors, 67, 8-17. https://doi.org/10.1016/j.addbeh.2016.11.024

Boyle, S. C., Earle, A. M., McCabe, N., \& LaBrie, J. W. (2018). Increasing chance-based uncertainty reduces heavy drinkers' cognitive reactance to web-based personalized normative feedback. Journal of Studies on Alcohol and Drugs, 79, 601-610. https://doi.org/10.15288/jsad.2018.79.601

Boyle, S. C., Kettering, V. L., Young, S., \& LaBrie, J. W. (2020). Lesbians' use of popular social media sites is associated with perceived drinking norms and interest in receiving PNF. Alcohol Treatment Quarterly, Advance online publication. https://doi.org/10.1080/07347324.2020.1723459

Boyle, S. C., LaBrie, J. W., Costine, L. D., \& Witkovic, Y. D. (2017). "It's how we deal”: Perceptions of LGB peers' use of alcohol and other drugs to cope and sexual minority adults' own coping motivated substance use following the Pulse nightclub shooting. Addictive Behaviors, 65, 51-55. https://doi.org/10.1016/j.addbeh.2016.10.001

Boyle, S. C., LaBrie, J. W., Omoto, A. M. (2020). Normative antecedents to substance use among sexual minorities: A scoping review and synthesis. Psychology of Sexual Orientation and Gender Diversity, 7, 117-131. https://doi.org/10.1037/sgd0000373

Boyle, S. C., LaBrie, J. W., \& Witkovic, Y. D. (2016). Do lesbians overestimate alcohol use norms? Exploring the potential utility of personalized normative feedback interventions to reduce high-risk drinking in Southern California lesbian communities. Journal of Gay \& Lesbian Social Services, 28, 179-194. https://doi.org/10.1080/10538720.2016.1190677 Brehm, J. W. (1966). A theory of psychological reactance. Academic Press. 
Burgard, S. A., Cochran, S. D., \& Mays, V. M. (2005). Alcohol and tobacco use patterns among heterosexually and homosexually experienced California women. Drug and Alcohol Dependence, 77, 61-70. https://doi.org/10.1016\%2Fj.drugalcdep.2004.07.007

Bush, R., Brown, R., McNair, R., Orellana, L., Lubman, D. I., \& Staiger, P. K. (2019). Effectiveness of a culturally tailored SMS alcohol intervention for same-sex attracted women: protocol for an RCT. BMC Women's Health, 19, e29. https://doi.org/10.1186/s12905-019-0729-y

Caceres, B. A., Brody, A., Luscombe, R. E., Primiano, J. E., Marusca, P., Sitts, E. M., \& Chyun, D. (2017). A systematic review of cardiovascular disease in sexual minorities. American Journal of Public Health, 107, e13-e21. https://doi.org/10.2105/AJPH.2016.303630

Campbell, M. K., \& Quintiliani, L. M. (2006). Tailored interventions in public health: Where does tailoring fit in interventions to reduce health disparities?. American Behavioral Scientist, 49, 775-793. https://doi.org/10.1177\%2F0002764205283807

Centerlink (2019). LGBT community center member directory. https://www.lgbtcenters.org Centers for Disease Control (2015). Excessive Drinking is Draining the U.S. Economy. https://www.cdc.gov/features/costsofdrinking/index.html

Chaudoir, S. R., Wang, K., \& Pachankis, J. E. (2015). What reduces sexual minority stress? A review of the intervention "toolkit." Journal of Social Issues, 73, 586-617. https://doi.org/10.1111/josi.12233

Cochran, B. N., \& Cauce, A. M. (2006). Characteristics of lesbian, gay, bisexual, and transgender individuals entering substance abuse treatment. Journal of Substance Abuse Treatment, 30, 135-146. https://doi.org/10.1016/j.jsat.2005.11.009 
Cochran, S. D., Grella, C. E., \& Mays, V. M. (2012). Do substance use norms and perceived drug availability mediate sexual orientation differences in patterns of substance use? Results from the California Quality of Life Survey II. Journal of Studies on Alcohol and Drugs, 73, 675-685. https://doi.org/10.15288/jsad.2012.73.675

Cogger, A., Conover, K. J., \& Israel, T. (2012). Factors influencing alcohol use among sexual minority women in a non-urban community: A mixed methods study. Journal of LGBT Issues in Counseling, 6, 293-309. https://doi.org/10.1080/15538605.2012.727745

Cohen, E., Feinn, R., Arias, A., \& Kranzler, H. R. (2007). Alcohol treatment utilization: findings from the National Epidemiologic Survey on Alcohol and Related Conditions. Drug and Alcohol Dependence, 86(2-3), 214-221.

Colbert, S., Thornton, L., \& Richmond, R. (2020). Smartphone apps for managing alcohol consumption: a literature review. Addiction Science \& Clinical Practice, 15, 1-16. https://doi.org/10.1186/s13722-020-00190-X

Collins S. E. (2016). Associations between socioeconomic factors and alcohol outcomes. Alcohol Research: Current Reviews, 38, 83-94.

Collins, S. E., Carey, K. B., \& Smyth, J. (2005). Relationships of linguistic and motivation variables with drinking outcomes following two mailed brief interventions. Journal of Studies on Alcohol, 66, 526-535. https://doi.org/10.15288/jsa.2005.66.526

Colwell, J. (2007). Needs met through computer game play among adolescents. Personality and Individual Differences, 43, 2072-2082. https://doi.org/10.1016/j.paid.2007.06.021

Conway, S., \& Elphinstone, B. (2017). Da-Sein design: Linking phenomenology with SelfDetermination Theory for game design. Journal of Gaming \& Virtual Worlds, 9, 55-69. https://doi.org/10.1386/jgvw.9.1.55_1 
Cooper, M. L. (1994). Motivations for alcohol use among adolescents: Development and validation of a four-factor model. Psychological Assessment, 6, 117-128. https://doi.org/10.1037/1040-3590.6.2.117

Coulter, R. W., Kinsky, S. M., Herrick, A. L., Stall, R. D., \& Bauermeister, J. A. (2015). Evidence of syndemics and sexuality-related discrimination among young sexualminority women. LGBT Health, 2, 250-257. https://doi.org/10.1089/lgbt.2014.0063

Coulter, R. W., Sang, J. M., Louth-Marquez, W., Henderson, E. R., Espelage, D., Hunter, S. C., ... Egan, J. E. (2019). Pilot testing the feasibility of a game intervention aimed at improving help seeking and coping among sexual and gender minority youth: Protocol for a randomized controlled trial. JMIR Research Protocols, 8, e12164. https://doi.org/10.2196/12164

Cox, S., \& Gallois, C. (1996). Gay and lesbian identity development: A social identity perspective. Journal of Homosexuality, 3, 1-30. https://doi.org/10.1300/J082v30n04_01

Cronce, J. M., Toomey, T. L., Lenk, K., Nelson, T. F., Kilmer, J. R., \& Larimer, M. E. (2018). NIAAA's College alcohol intervention matrix: CollegeAIM. Alcohol Research: Current Reviews, 39, 43-47.

Cugelman, B. (2013). Gamification: What it is and why it matters to digital health behavior change developers. JMIR Serious Games, 1, e3. http://dx.doi.org/10.2196/games.3139.

Curran, G. M., Bauer, M., Mittman, B., Pyne, J. M., \& Stetler, C. (2012). Effectivenessimplementation hybrid designs. Medical Care, 50, 217-226. https://doi.org/10.1097/MLR.0b013e3182408812 
Davis-Delano, L. R. (2014). Sport as context for the development of women's same-sex relationships. Journal of Sport and Social Issues, 38, 263-285. https://doi.org/10.1177\%2F0193723513520554

Deci, E. L., \& Ryan, R. M. (2004). Handbook of Self-determination Research. Rochester, NY: University of Rochester Press.

Deci, E. L., \& Ryan, R. M. (2008). Self-determination theory: A macrotheory of human motivation, development, and health. Canadian Psychology/Psychologie Canadienne, 49, 182-185. https://doi.org/10.1037/a0012801

Deci, E. L., Ryan, R. M., Weiner, I. B., \& Craighead, W. E. (2010). Self-determination. In The Corsini Encyclopedia of Psychology (Vol. 1-2). Hoboken, NJ: John Wiley \& Sons.

Dempsey, R. C., McAlaney, J., \& Bewick, B. M. (2018). A critical appraisal of the social norms approach as an interventional strategy for health-related behavior and attitude change. Frontiers in Psychology, 9. https://doi.org/10.3389/fpsyg.2018.02180

DeSmet, A., Shegog, R., Van Ryckeghem, D., Crombez, G., \& De Bourdeaudhuij, I. (2015). A systematic review and meta-analysis of interventions for sexual health promotion involving serious digital games. Games for Health Journal, 4, 78-90. https://doi.org/10.1089/g4h.2014.0110

Devine, P. G. (1989). Stereotypes and prejudice: Their automatic and controlled components. Journal of Personality and Social Psychology, 56(1), 518. https://doi.org/10.1037/0022-3514.56.1.5

Dillard, J. P., \& Shen, L. (2005). On the nature of reactance and its role in persuasive health communication. Communication Monographs, 72,144-168. https://doi.org/10.1080/03637750500111815 
Dolance, S. (2005). “A whole stadium full”: Lesbian community at women's national basketball association games. Journal of Sex Research, 42, 74-83. https://doi.org/10.1080/00224490509552259

Dorn-Medeiros, C. M., \& Doyle, C. (2018). Alcohol as coping: Internalized homophobia and heterosexism's role in alcohol use among lesbians. Journal of LGBT Issues in Counseling, 12, 142-157. https://doi.org/10.1080/15538605.2018.1488230

Dotson, K. B., Dunn, M. E., \& Bowers, C. A. (2015). Stand-alone personalized normative feedback for college student drinkers: A meta-analytic review, 2004 to 2014. PLOS ONE, 10, e0139518. https://doi.org/10.1371/journal.pone.0139518

Doumas, D. M., Esp, S., Turrisi, R., Hausheer, R., \& Cuffee, C. (2014). A test of the efficacy of a brief, web-based personalized feedback intervention to reduce drinking among 9th grade students. Addictive Behaviors, 39, 231-238. https://doi.org/10.1016/j.addbeh.2013.10.011

Drabble, L., \& Trocki, K. (2005). Alcohol consumption, alcohol-related problems, and other substance use among lesbian and bisexual women. Journal of Lesbian Studies, 9, 19-30. https://doi.org/10.1300/J155v09n03_03

Drabble, L., \& Trocki, K. (2014). Alcohol in the life narratives of women: Commonalities and differences by sexual orientation. Addiction Research \& Theory, 22, 186194. https://doi.org/10.3109/16066359.2013.806651

Dworkin, E. R., Cadigan, J., Hughes, T., Lee, C., \& Kaysen, D. (2018). Sexual identity of drinking companions, drinking motives, and drinking behaviors among young sexual minority women: An analysis of daily data. Psychology of Addictive Behaviors, 32, 540-551. https://doi.org/10.1037/adb0000384 
Earle, A. M., LaBrie, J. W., Boyle, S. C., \& Smith, D. (2018). In pursuit of a self-sustaining college alcohol intervention: Deploying gamified PNF in the real world. Addictive Behaviors, 80, 71-81. https://doi.org/10.1016/j.addbeh.2018.01.005

Edney, S. M., Olds, T. S., Ryan, J. C., Vandelanotte, C., Plotnikoff, R. C., Curtis, R. G., \& Maher, C. A. (2020). A Social Networking and Gamified App to Increase Physical Activity: Cluster RCT. American Journal of Preventive Medicine, 58, e51-e62.

Ehlke, S. J., Stamates, A. L., Kelley, M. L., \& Braitman, A. L. (2019). Bisexual women's reports of descriptive drinking norms for heterosexual, bisexual, and lesbian women. Psychology of Sexual Orientation and Gender Diversity, 6, 256-263. https://doi.org/10.1037/sgd0000312

El-Hilly, A. A., Iqbal, S. S., Ahmed, M., Sherwani, Y., Muntasir, M., Siddiqui, S.,... \& Eisingerich, A. B. (2016). Game on? Smoking cessation through the gamification of mHealth: A longitudinal qualitative study. JMIR Serious Games, 4, e18. https://doi.org/10.2196/games.5678

Escobar, K. M., \& Gorey, K. M. (2018). Cognitive behavioral interventions for depression among Hispanic people: promising meta-analytic evidence for deep cultural adaptations. Social Work in Mental Health, 16, 746-758. https://doi.org/10.1080/15332985.2018.1476284

Everett, B. G., Hatzenbuehler, M. L., \& Hughes, T. L. (2016). The impact of civil union legislation on minority stress, depression, and hazardous drinking in a diverse sample of sexual-minority women: A quasi-natural experiment. Social Science \& Medicine, 169, 180-190. https://doi:10.1016/j.socscimed.2016.09.036 
Farmer, G. W., Jabson, J. M., Bucholz, K. K., \& Bowen, D. J. (2013). A population-based study of cardiovascular disease risk in sexual-minority women. American Journal of Public Health, 103, 1845-1850. https://doi.org/10.2105/AJPH.2013.301258

Feinstein, B. A., Dyar, C., \& London, B. (2017). Are outness and community involvement risk or protective factors for alcohol and drug abuse among sexual minority women? Archives of Sexual Behavior, 46, 1411-1423. https://doi.org/10.1007/s10508-016-0790-7.

Fingerhut, A. W., \& Abdou, C. M. (2017). The role of healthcare stereotype threat and social identity threat in LGB health disparities. Journal of Social Issues, 73(3), 493-507. https://doi.org/10.1111/josi.12228

Fish, J. N. (2019). Sexual orientation-related disparities in high-intensity binge drinking: Findings from a nationally representative sample. LGBT Health, 6, 242-249. https://doi.org/10.1089/lgbt.2018.0244

Fishbein, M., \& Ajzen, I. (2011). Predicting and changing behavior: The reasoned action approach. New York, NY: Taylor \& Francis.

Fisher, D. A., Hill, D. L., Grube, J. W., \& Gruber, E. L. (2007). Gay, lesbian, and bisexual content on television: A quantitative analysis across two seasons. Journal of Homosexuality, 52, 167-188. https://doi.org/10.1300/J082v52n03_08

Fitzpatrick, S., Dworkin, E. R., Zimmerman, L., Javorka, M., \& Kaysen, D. (2020). Stressors and drinking in sexual minority women: The mediating role of emotion dysregulation. Psychology of Sexual Orientation and Gender Diversity, 7, 4654. https://doi.org/10.1037/sgd0000351

Flanders, C. E., Pragg, L., Dobinson, C., \& Logie, C. (2017). Young sexual minority women's 
use of the internet and other digital technologies for sexual health information seeking. The Canadian Journal of Human Sexuality, 26, 17-25. https://doi.org/10.3138/cjhs.261-A2

Fleming, T. M., Bavin, L., Stasiak, K., Hermansson-Webb, E., Merry, S. N., Cheek, C.,... \& Hetrick, S. (2017). Serious games and gamification for mental health: current status and promising directions. Frontiers in Psychiatry, 7, 215. https://doi.org/10.3389/fpsyt.2016.00215

Flores-Aranda, J., Goyette, M., \& Larose-Osterrath, C. (2019). Online intervention as strategy to reach men who have sex with other men and who use substances in a sexual context: Development of the MONBUZZ.ca Project. Frontiers in Psychiatry, 10. https://doi.org/10.3389/fpsyt.2019.00183

Floryan, M. R., Ritterband, L. M., \& Chow, P. I. (2019). Principles of gamification for Internet interventions. Translational Behavioral Medicine, 9, 1131-1138. https://doi.org/10.1093/tbm/ibz041

Folkman, S., Chesney, M., McKusick, L., Ironson, G., Johnson, D. S., \& Coates, T. J. (1991). Translating coping theory into an intervention. In The social context of coping (pp. 239260). Springer, Boston, MA.

Folkman, S., \& Lazarus, R. S. (1984). Stress, appraisal, and coping. New York, NY: Springer Publishing Company.

Fox, J., \& Warber, K. M. (2015). Queer identity management and political self-expression on social networking sites: A co-cultural approach to the spiral of silence. Journal of Communication, 65, 79-100. https://doi.org/10.1111/jcom.12137 
Fredriksen-Goldsen, K. I., Hoy-Ellis, C. P., \& Brown, M. (2015). Addressing behavioral cancer risks from a LGBT health equity perspective. In U. Boehmer \& R. Elk (Eds.), Cancer and the LGBT Community: Unique Perspectives from Risk to Survivorship (pp. 37-62). https://doi.org/10.1007/978-3-319-15057-4_4

Gabbay, S. G., \& Wahler, J. J. (2002). Lesbian aging. Journal of Gay \& Lesbian Social Services, 14, 1-21. https://doi.org/10.1300/J041v14n03_01

Gardner, L., \& Leshner, G. (2016). The role of narrative and other referencing in attenuating psychological reactance to diabetes self-care messages. Health Communication, 31, 738751. https://doi.org/10.1080/10410236.2014.993498

Germanos, R., Deacon, R., \& Mooney-Somers, J. (2015). The social and cultural significance of women's sexual identities should guide health promotion. LGBT Health, 2, 162-168. https://doi.org/10.1089/lgbt.2014.0118

Ghabrial, M. A. (2019). "We can shapeshift and build bridges": Bisexual women and gender diverse people of color on invisibility and embracing the borderlands. Journal of Bisexuality, 19, 169-197. https://doi.org/10.1080/15299716.2019.1617526

Gillison, F. B., Rouse, P., Standage, M., Sebire, S. J., \& Ryan, R. M. (2019). A meta-analysis of techniques to promote motivation for health behaviour change from a self-determination theory perspective. Health Psychology Review, 13, 110-130. https://doi.org/10.1080/17437199.2018.1534071

Granfield, R. (2005). Alcohol use in college: Limitations on the transformation of social norms. Addiction Research \& Theory, 13, 281-292. https://doi.org/10.1080/16066350500053620 
Grant, B. F. (1997). Barriers to alcoholism treatment: reasons for not seeking treatment in a general population sample. Journal of Studies on Alcohol, 58, 365-371. https://doi.org/10.15288/jsa.1997.58.365

Grant, B. F., Goldstein, R. B., Saha, T. D., Chou, S. P., Jung, J., Zhang, H.,... \& Hasin, D. S. (2015). Epidemiology of DSM-5 alcohol use disorder: results from the National Epidemiologic Survey on Alcohol and Related Conditions III. JAMA Psychiatry, 72, 757766. https://doi.org/10.1001/jamapsychiatry.2015.0584

Goldenberg, J. L., \& Arndt, J. (2008). The implications of death for health: A terror management health model for behavioral health promotion. Psychological Review, 115, 10321053. https://doi.org/10.1037/a0013326

Gordon, L. E. (2006). Bringing the U-Haul: Embracing and resisting sexual stereotypes in a lesbian community. Sexualities, 9(2), 171-192. https://doi.org/10.1177\%2F1363460706063118

Green, K. E. (2011). Barriers and treatment preferences reported by worried drinkers of various sexual orientations. Alcoholism Treatment Quarterly, 29, 45-63. https://doi.org/10.1080/07347324.2011.538311

Green, K. E., \& Feinstein, B. A. (2012). Substance use in lesbian, gay, and bisexual populations: An update on empirical research and implications for treatment. Psychology of Addictive Behaviors, 26, 265-278. https://doi.org/10.1037/a0025424

Greenberg, J., Pyszczynski, T., \& Solomon, S. (1986). The causes and consequences of a need for self-esteem: A terror management theory. In R. F. Baumeister (Ed.), Public Self and Private Self (pp. 189-212). https://doi.org/10.1007/978-1-4613-9564-5_10 
Greenberg, J., Solomon, S., \& Pyszczynski, T. (1997). Terror management theory of self-esteem and cultural worldviews: Empirical assessments and conceptual refinements. In M. P. Zanna (Ed.), Advances in Experimental Social Psychology (Vol. 29, pp. 61-139). https://doi.org/10.1016/S0065-2601(08)60016-7

Grella, C. E., Greenwell, L., Mays, V. M., \& Cochran, S. D. (2009). Influence of gender, sexual orientation, and need on treatment utilization for substance use and mental disorders: Findings from the California Quality of Life Survey. BMC Psychiatry, 9, 52. https://doi.org/10.1186/1471-244X-9-52

Gruskin, E., Byrne, K., Kools, S., \& Altschuler, A. (2007). Consequences of frequenting the lesbian bar. Women \& Health, 44, 103-120. https://doi.org/10.1300/j013v44n02_06

Hall, G. C. N., Ibaraki, A. Y., Huang, E. R., Marti, C. N., \& Stice, E. (2016). A meta-analysis of cultural adaptations of psychological interventions. Behavior Therapy, 47(6), 993-1014. https://doi.org/10.1016/j.beth.2016.09.005

Hallgren, K. A., \& Witkiewitz, K. (2013). Missing data in alcohol clinical trials: a comparison of methods. Alcoholism: Clinical and Experimental Research, 37, 2152-2160. https://doi.org/10.1111\%2Facer.12205

Hamilton, C. J., \& Mahalik, L. R. (2009). Minority stress, masculinity, and social norms predicting gay men's health risk behaviors. Journal of Counseling Psychology, 56, 132141. https://doi.org/10.1037/a0014440

Hansen, W. B., \& Scheier, L. M. (2019). Specialized smartphone intervention apps: review of 2014 to 2018 NIH funded grants. JMIR mHealth and uHealth, 7, e14655. https://doi.org/10.2196/14655

Hartman, J. E. (2008). Another kind of "Chilly Climate" The effects of lesbian separatism on 
bisexual women's identity and community. Journal of Bisexuality, 5, 61-76. https://doi.org/10.1300/J159v05n04_06

Hatzenbuehler, M. L. (2009). How does sexual minority stigma "get under the skin"? A psychological mediation framework. Psychological Bulletin, 135, 707-730. https://doi.org/10.1037/a0016441

Hatzenbuehler, M. L. (2014). Structural stigma and the health of lesbian, gay, and bisexual populations. Current Directions in Psychological Science, 23, 127-132. https://doi.org/10.1177/0963721414523775

Hatzenbuehler, M. L. (2016). Structural stigma: Research evidence and implications for psychological science. American Psychologist, 71, 742-751. https://doi.org/10.1037/amp0000068.

Hatzenbuehler, M. L., Jun, H.-J., Corliss, H. L., \& Austin, S. B. (2015). Structural stigma and sexual orientation disparities in adolescent drug use. Addictive Behaviors, 46, 14-18. https://doi.org/10.1016/j.addbeh.2015.02.017

Hatzenbuehler, M. L., \& Keyes, K. M. (2013). Inclusive anti-bullying policies and reduced risk of suicide attempts in lesbian and gay youth. Journal of Adolescent Health, 53, 21-26. https://doi.org/10.1016/j.jadohealth.2012.08.010

Hatzenbuehler, M. L., \& Link, B. G. (2014). Introduction to the special issue on structural stigma and health. Social Science \& Medicine, 103, 1-6. https://doi.org/10.1016/j.socscimed.2013.12.017

Hayes, A. F. (2017). Introduction to mediation, moderation, and conditional process analysis: A regression-based approach. New York: NY: Guilford Publications. 
Herrera, A. P. (2018). Theorizing the lesbian hashtag: Identity, community, and the technological imperative to name the sexual self. Journal of Lesbian Studies, 22, 313-328. https://doi.org/10.1080/10894160.2018.1384263

Hester, R. K., Squires, D. D., \& Delaney, H. D. (2005). The Drinker’s Check-up: 12-month outcomes of a controlled clinical trial of a stand-alone software program for problem drinkers. Journal of Substance Abuse Treatment, 28, 159-169. https://doi.org 10.1016/j.jsat.2004.12.002.

Hightow-Weidman, L. B., Muessig, K. E., Bauermeister, J. A., LeGrand, S., \& Fiellin, L. E. (2017). The future of digital games for HIV prevention and care. Current Opinion in HIV and AIDS, 12, 501-507. https://doi.org/10.1097/COH.0000000000000399

Hilbe, J. M. (2014). Modeling count data. Cambridge, UK: Cambridge University Press.

Hogg, M. A., \& Reid, S. A. (2006). Social identity, self-categorization, and the communication of group norms. Communication Theory, 16, 7-30. https://doi.org/10.1111/j.14682885.2006.00003.x

Hogg, M. A., \& Turner, J. C. (1987). Intergroup behaviour, self-stereotyping and the salience of social categories. British Journal of Social Psychology, 26(4), 325-340. https://doi.org/10.1111/j.2044-8309.1987.tb00795.x

Hou, J. (2011). Uses and gratifications of social games: Blending social networking and game play. First Monday, 16. https://doi.org/10.5210/fm.v16i7.3517

Hughes, T. (2011). Alcohol use and alcohol-related problems among sexual minority women. Alcoholism Treatment Quarterly, 29, 403-435. https://doi.org/10.1080\%2F07347324.2011.608336 
Hughes, T. L., Veldhuis, C. B., Drabble, L. A., \& Wilsnack, S. C. (2020). Research on alcohol and other drug (AOD) use among sexual minority women: A global scoping review. PLoS One, 15, e0229869. https://doi.org/10.1371/journal.pone.0229869

Hughes, T. L., Wilsnack, S. C., Szalacha, L. A., Johnson, T., Bostwick, W. B., Seymour, R.,... \& Kinnison, K. E. (2006). Age and racial/ethnic differences in drinking and drinking-related problems in a community sample of lesbians. Journal of Studies on Alcohol, 67(4), 579590. https://doi.org/10.15288/jsa.2006.67.579

Hummer, J. F., \& Davison, G. C. (2016). Examining the role of source credibility and reference group proximity on personalized normative feedback interventions for college student alcohol use: A randomized laboratory experiment. Substance Use \& Misuse, 51, 17011715. https://doi.org/10.1080/10826084.2016.1197258

Hummer, J. F., Hatch, M. R., \& Davison, G. C. (2020). Cognitive-affective change mechanisms in personalized normative feedback via the articulated thoughts in simulated situations paradigm. International Journal of Environmental Research and Public Health, 17, 690. https://doi.org/10.3390/ijerph17030690.

Huxley, C., Clarke, V., \& Halliwell, E. (2014). Resisting and conforming to the 'lesbian look': The importance of appearance norms for lesbian and bisexual women. Journal of Community \& Applied Social Psychology, 24, 205-219. https://doi.org/10.1002/casp.2161

Johnson, D., Deterding, S., Kuhn, K.-A., Staneva, A., Stoyanov, S., \& Hides, L. (2016). Gamification for health and wellbeing: A systematic review of the literature. Internet Interventions, 6, 89-106. https://doi.org/10.1016/j.invent.2016.10.002 
Jonas, E., \& Fritsche, I. (2012). Follow the norm! Terror management theory and the influence of descriptive norms. Social Psychology, 43, 28-32. https://doi.org/10.1027/18649335/a000077

Jonas, E., Martens, A., Niesta Kayser, D., Fritsche, I., Sullivan, D., \& Greenberg, J. (2008). Focus theory of normative conduct and terror-management theory: The interactive impact of mortality salience and norm salience on social judgment. Journal of Personality and Social Psychology, 95, 1239-1251. https://doi.org/10.1037/a0013593

Kalb, N., Roy Gillis, J., \& Goldstein, A. L. (2018). Drinking to cope with sexual minority stressors: Understanding alcohol use and consequences among LGBQ emerging adults. Journal of Gay \& Lesbian Mental Health, 22, 310-326. https://doi.org/10.1080/19359705.2018.1476277

Kapp, K. M. (2013). The gamification of learning and instruction field book: Ideas into practice. New York, NY: John Wiley \& Sons.

Kato, P. M., Cole, S. W., Bradlyn, A. S., \& Pollock, B. H. (2008). A video game improves behavioral outcomes in adolescents and young adults with cancer: a randomized trial. Pediatrics, 122, e305-e317. https://doi.org/10.1542/peds.2007-3134

Kawachi, I. (2017). It's all in the game-The uses of gamification to motivate behavior change. JAMA Internal Medicine, 177, 1593-1594. https://doi.org/10.1001/jamainternmed.2017.4798

Kelly, R. B., Zyzanski, S. J., \& Alemagno, S. A. (1991). Prediction of motivation and behavior change following health promotion: Role of health beliefs, social support, and selfefficacy. Social Science \& Medicine, 32, 311-320. https://doi.org/10.1016/027795369190109-P 
Keyes, K. M., Liu, X. C., \& Cerda, M. (2012). The role of race/ethnicity in alcohol-attributable injury in the United States. Epidemiologic Reviews, 34, 89-102. https://doi.org/10.1093/epirev/mxr018

King, D., Greaves, F., Exeter, C., \& Darzi, A. (2013). 'Gamification': Influencing health behaviours with games. Journal of the Royal Society of Medicine, 106, 76-78. https://doi.org/10.1177/0141076813480996

Knapton, H. M., Bäck, H., \& Bäck, E. A. (2015). The social activist: Conformity to the ingroup following rejection as a predictor of political participation. Social Influence, 10, 97-108. https://doi.org/10.1080/15534510.2014.966856

Kohl L. F., Crutzen R., de Vries N. K. (2013). Online prevention aimed at lifestyle behaviors: A systematic review of reviews. Journal of Medical Internet Research, 15, e146, https://doi.org/10.2196/jmir.2665

Koivisto, J., \& Hamari, J. (2014). Demographic differences in perceived benefits from gamification. Computers in Human Behavior, 35, 179-188. https://doi.org/10.1016/j.chb.2014.03.007

Kreuter, M. W., \& Haughton, L. T. (2006). Integrating culture into health information for African American women. American Behavioral Scientist, 49, 794-811. https://doi.org/10.1177\%2F0002764205283801

Kuerbis, A. N., Schaumberg, K., Davis, C. M., Hail, L., \& Morgenstern, J. (2014). Unpacking personalized feedback: An exploratory study of the impact of its components and the reactions it elicits among problem drinking men who have sex with men. Substance Use \& Misuse, 49, 383-394. https://doi.org/10.3109/10826084.2013.841247 
LaBrie, J. W., de Rutte, J. L., Boyle, S. C., Tan, C. N., \& Earle, A. M. (2019). Leveraging copresence to increase the effectiveness of gamified personalized normative feedback. Addictive Behaviors, 99, 113-125. https://doi.org/10.1016/j.addbeh.2019.106085

LaBrie, J. W., Lewis, M. A., Atkins, D. C., Neighbors, C., Zheng, C., Kenney, S. R.,... \& Grossbard, J. (2013). RCT of web-based personalized normative feedback for college drinking prevention: are typical student norms good enough? Journal of Consulting and Clinical Psychology, 81, 1074-1086 https://doi.org/10.1037\%2Fa0034087

Landes, S. J., McBain, S. A., \& Curran, G. M. (2019). An introduction to effectivenessimplementation hybrid designs. Psychiatry Research, 280, 112513. https://doi.org/10.1016/j.psychres.2019.112513

Larimer, M. E., Kaysen, D. L., Lee, C. M., Kilmer, J. R., Lewis, M. A., Dillworth, T.,... \& Neighbors, C. (2009). Evaluating level of specificity of normative referents in relation to personal drinking behavior. Journal of Studies on Alcohol and Drugs, 16, 115-121. doi:10.15288/jsads.2009.s16.115.

Lax, J. R., \& Phillips, J. H. (2009). Gay rights in the states: Public opinion and policy responsiveness. American Political Science Review, 103, 367-386. https://doi.org/10.1017/S0003055409990050

LGBT Demographic Data Interactive. (2019). The Williams Institute, UCLA School of Law. Available at: https://williamsinstitute.law.ucla.edu/visualization/lgbtstats/?topic=SS\&showCounties=true\#density

Lehavot, K., \& Simoni, J. M. (2011). The impact of minority stress on mental health and substance use among sexual minority women. Journal of Consulting and Clinical Psychology, 79, 159-170. https://doi.org/10.1037/a0022839 
Lenhard, W., \& Lenhard, A. (2016). Calculation of Effect Sizes. Available at https://www.psychometrica.de/effect_size.html.

Levak, S., Kuerbis, A. N., \& Morgenstern, J. (2020). Drink goal difficulty effect on outcomes in moderation-based alcohol treatment for sexual minority men. Journal of Substance Abuse Treatment. Online first version. https://doi.org/10.1016/j.jsat.2020.01.001

Lewis, M. A., \& Neighbors, C. (2015). An examination of college student activities and attentiveness during a web-delivered personalized normative feedback intervention. Psychology of Addictive Behaviors, 29, 162-167. https://doi.org/10.1037/adb0000003

Lewis, R. J., Ehlke, S. J., Shappie, A. T., Braitman, A. L., \& Heron, K. E. (2019). Health disparities among exclusively lesbian, mostly lesbian, and bisexual young women. $L G B T$ Health, 6, 400-408. https://doi.org/10.1089/lgbt.2019.0055

Lewis, R. J., Winstead, B. A., Lau-Barraco, C., \& Mason, T. B. (2017). Social factors linking stigma-related stress with alcohol use among lesbians. Journal of Social Issues, 73, 545562. https://doi.org/10.1111/josi.12231

Lewis, R. J., Mason, T. B., Winstead, B. A., Gaskins, M., \& Irons, L. B. (2016). Pathways to hazardous drinking among racially and socioeconomically diverse lesbian women: Sexual minority stress, rumination, social isolation, and drinking to cope. Psychology of Women Quarterly, 40, 564-581. https://doi.org/10.1177\%2F0361684316662603

LGBT Demographic Data Interactive. (2019). The Williams Institute, UCLA School of Law. Available at: https://williamsinstitute.law.ucla.edu/visualization/lgbtstats/?topic=SS\&showCounties=true\#density

Liang, R.-H. (2012). Designing for unexpected encounters with digital products: Case studies of serendipity as felt experience. International Journal of Design, 6, 41-58. 
Lick, D. J., Durso, L. E., \& Johnson, K. L. (2013). Minority stress and physical health among sexual minorities. Perspectives on Psychological Science, 8(5), 521-548. https://doi.org/10.1177/1745691613497965

Lim, S., \& Reeves, B. (2010). Computer agents versus avatars: Responses to interactive game characters controlled by a computer or other player. International Journal of HumanComputer Studies, 68, 57-68. https://doi.org/10.1016/j.ijhcs.2009.09.008

Lipsky, S., Krupski, A., Roy-Byrne, P., Huber, A., Lucenko, B. A., \& Mancuso, D. (2012). Impact of sexual orientation and co-occurring disorders on chemical dependency treatment outcomes. Journal of Studies on Alcohol and Drugs, 73, 401-412. https://doi.org/10.15288/jsad.2012.73.401

Litt, D. M., Lewis, M. A., Rhew, I. C., Hodge, K. A., \& Kaysen, D. L. (2015). Reciprocal relationships over time between descriptive norms and alcohol use in young adult sexual minority women. Psychology of Addictive Behaviors, 29, 885-893. https://doi.org/10.1037/adb0000122

Liu, J., Zhao, S., Chen, X., Falk, E., \& Albarracín, D. (2017). The influence of peer behavior as a function of social and cultural closeness: A meta-analysis of normative influence on adolescent smoking initiation and continuation. Psychological Bulletin, 143, 1082-1115. https://doi.org/10.1037\%2Fbu10000113.

Liu, L. (2016). Using generic inductive approach in qualitative educational research: A case study analysis. Journal of Education and Learning, 5, 129-135. https://doi.org/10.5539/jel.v5n2p129 
Mandryk, R. L., Inkpen, K. M., \& Calvert, T. W. (2006). Using psychophysiological techniques to measure user experience with entertainment technologies. Behaviour \& Information Technology, 25, 141-158. https://doi.org/10.1080/01449290500331156

Mason, T. B., \& Lewis, R. J. (2014). Reducing obesity among lesbian women: Recommendations for culturally tailored interventions. Psychology of Sexual Orientation and Gender Diversity, 1, 361-376. https://doi.org/10.1037/sgd0000074

Matsick, J. L., \& Rubin, J. D. (2018). Bisexual prejudice among lesbian and gay people: Examining the roles of gender and perceived sexual orientation. Psychology of Sexual Orientation and Gender Diversity, 5, 143-155. https://doi.org/10.1037/sgd0000283

May, C., \& Nielsen, A. S. (2019). Barriers to Treatment for Alcohol Dependence. Journal of Drug and Alcohol Research, 8, 1-17. https://doi.org/10.4303/jdar/236083

McCabe, S. E., Hughes, T. L., Bostwick, W. B., West, B. T., \& Boyd, C. J. (2009). Sexual orientation, substance use behaviors and substance dependence in the United States. Addiction, 104, 1333-1345. https://doi.org/10.1111/j.1360-0443.2009.02596.x

McCabe, S. E., West, B. T., Hughes, T. L., \& Boyd, C. J. (2013). Sexual orientation and substance abuse treatment utilization in the United States: Results from a national survey. Journal of Substance Abuse Treatment, 44, 4-12. https://doi.org/10.1016/j.jsat.2012.01.007

McInroy, L.B. \& Craig, S.L. (2017). Perspectives of LGBTQ emerging adults on the depiction and impact of LGBTQ media representation. Journal of Youth Studies, 20, 32-46. https://10.1080/13676261.2016.1184243

McGovern, T. (2018). County-level 2016 election data from TownHall.com. Available at: 
https://github.com/tonmcg/US_County_Level_Election_Results_08-16

/blob/master/2016_US_County Level_Presidential_Results.csv

McNair, R., Pennay, A., Hughes, T., Brown, R., Leonard, W., \& Lubman, D. I. (2016). A model for lesbian, bisexual and queer-related influences on alcohol consumption and implications for policy and practice. Culture, Health \& Sexuality, 18, 405-421. https://doi.org/10.1080/13691058.2015.1089602

Mekler, E. D., Brühlmann, F., Tuch, A. N., \& Opwis, K. (2017). Towards understanding the effects of individual gamification elements on intrinsic motivation and performance. Computers in Human Behavior, 71, 525-534. https://doi.org/10.1016/j.chb.2015.08.048

Mejia, C. M., Acland, D., Buzdugan, R., Grimball, R., Natoli, L., McGrath, M. R., .. McCoy, S. I. (2017). An intervention using gamification to increase human immunodeficiency virus and sexually transmitted infection screening among young men who have sex with men in California: Rationale and design of Stick To It. JMIR Research Protocols, 6, e140. https://doi.org/10.2196/resprot.8064

Meyer, I. H. (2003). Prejudice, social stress, and mental health in lesbian, gay, and bisexual populations: Conceptual issues and research evidence. Psychological Bulletin, 129, 674697. https://doi.org/10.1037/0033-2909.129.5.674

Millar, B. M., Wang, K., \& Pachankis, J. E. (2016). The moderating role of internalized homonegativity on the efficacy of LGB-affirmative psychotherapy: Results from a randomized controlled trial with young adult gay and bisexual men. Journal of Consulting and Clinical Psychology, 84, 565-570. https://doi.org/10.1037\%2Fccp0000113 
Miller, D. T., \& Prentice, D. A. (2016). Changing norms to change behavior. Annual Review of Psychology, 67, 339-361. https://doi.org/10.1146/annurev-psych-010814-015013

Miller, M. B., \& Leffingwell, T. R. (2013). What do college student drinkers want to know? Student perceptions of alcohol-related feedback. Psychology of Addictive Behaviors, 27, 214-222. https://doi.org/10.1037/a0031380

Milne-Ives, M., Lam, C., De Cock, C., Van Velthoven, M. H., \& Meinert, E. (2020). Mobile apps for health behavior change in physical activity, diet, drug and alcohol use, and mental health: Systematic review. JMIR mHealth and uHealth, 8, e17046. https://doi.org/10.2196/17046

Mock, S. E., Misener, K., \& Havitz, M. E. (2019). A league of their own? A longitudinal study of ego involvement and participation behaviors in lgbt-focused community sport. Leisure Sciences, 1-18. https://doi.org/10.1080/01490400.2019.1665599

Mokdad, A. H., Ballestros, K., Echko, M., Glenn, S., Olsen, H. E., Mullany, E.,... \& Kasaeian, A. (2018). The state of US health, 1990-2016: burden of diseases, injuries, and risk factors among US states. JAMA, 319, 1444-1472. https://doi.org/10.1001/jama.2018.0158

Morris, B. (2016). The Disappearing L: Erasure of Lesbian Spaces and Culture, New York, NY: SUNY Press.

Morris, S. B. (2008). Estimating effect sizes from pretest-posttest-control group designs. Organizational Research Methods, 11, 364-386. https://doi.org/10.1177\%2F1094428106291059

Movement Advancement Project. (2019). Interactive equality maps: State data table. Available at: http://www.lgbtmap.org/equality-maps 
Mulia, N., Tam, T. W., \& Schmidt, L. A. (2014). Disparities in the use and quality of alcohol treatment services and some proposed solutions to narrow the gap. Psychiatric Services, 65, 626-633. https://doi.org/10.1176/appi.ps.201300188

Murphy, J. G., Benson, T. A., Vuchinich, R. E., Deskins, M. M., Eakin, D., Flood, A. M.,... \& Torrealday, O. (2004). A comparison of personalized feedback for college student drinkers delivered with and without a motivational interview. Journal of Studies on Alcohol, 65, 200-203. https://doi.org/10.15288/jsa.2004.65.200

Murphy, J. G., Dennhardt, A. A., Skidmore, J. R., Martens, M. P., \& McDevitt-Murphy, M. E. (2010). Computerized versus motivational interviewing alcohol interventions: impact on discrepancy, motivation, and drinking. Psychology of Addictive Behaviors, 24, 628-639. https://doi.org/10.1037/a0021347

National Institute on Alcohol Abuse \& Alcoholism (2020). Alcohol facts and statistics. https://www.niaaa.nih.gov/publications/brochures-and-fact-sheets/alcohol-facts-andstatistics

Nawyn, S. J., Richman, J. A., Rospenda, K. M., \& Hughes, T. L. (2000). Sexual identity and alcohol-related outcomes: Contributions of workplace harassment. Journal of Substance Abuse, 11, 289-304. https://doi.org/10.1016/S0899-3289(00)00028-6

Neighbors, C., LaBrie, J. W., Hummer, J. F., Lewis, M. A., Lee, C. M., Desai, S.,... Kilmer, J. R., \& Larimer, M. E. (2010). Group identification as a moderator of the relationship between perceived social norms and alcohol consumption. Psychology of Addictive Behaviors, 24, 522-528. https://doi.org/10.1037/a0019944

Neighbors, C., Larimer, M. E., \& Lewis, M. A. (2004). Targeting misperceptions of descriptive drinking norms: efficacy of a computer-delivered personalized normative feedback 
intervention. Journal of Consulting and Clinical Psychology, 72, 434-447.

https://doi.org/10.1037/0022-006X.72.3.434

Neighbors, C., Walker, D., Rodriguez, L., Walton, T., Mbilinyi, L., Kaysen, D., \& Roffman, R. (2014). Normative misperceptions of alcohol use among substance abusing army personnel. Military Behavioral Health, 2(2), 203-209.

https://doi.org/10.1080/21635781.2014.890883

Okamoto, S. K., Kulis, S., Marsiglia, F. F., Steiker, L. K. H., \& Dustman, P. (2014). A continuum of approaches toward developing culturally focused prevention interventions: From adaptation to grounding. The Journal of Primary Prevention, 35, 103-112. https://doi.org/10.1007/s10935-013-0334-z

Oldenburg, C. E., Perez-Brumer, A. G., Hatzenbuehler, M. L., Krakower, D., Novak, D. S., Mimiaga, M. J., \& Mayer, K. H. (2015). State-level structural sexual stigma and HIV prevention in a national online sample of HIV-uninfected men who have sex with men in the United States. AIDS, 29, 837-845. https://doi.org/10.1097\%2FQAD.0000000000000622

Ozcelik, E., Cagiltay, N. E., \& Ozcelik, N. S. (2013). The effect of uncertainty on learning in game-like environments. Computers \& Education, 67, 12-20. https://doi.org/10.1016/j.compedu.2013.02.009

Pachankis, J. E., Hatzenbuehler, M. L., \& Starks, T. J. (2014). The influence of structural stigma and rejection sensitivity on young sexual minority men's daily tobacco and alcohol use. Social Science \& Medicine, 103, 67-75. https://doi.org/10.1016/j.socscimed.2013.10.005 
Pachankis, J. E., McConocha, E. M., Clark, K. A., Wang, K., Behari, K., Fetzner, B. K., Brisbin, C. D., Scheer, J. R., \& Lehavot, K. (2020). A transdiagnostic minority stress intervention for gender diverse sexual minority women's depression, anxiety, and unhealthy alcohol use: A randomized controlled trial. Journal of Consulting and Clinical Psychology, 88(7), 613-630. https://doi.org/10.1037/ccp0000508

Pachankis, J. E., McConocha, E. M., Reynolds, J. S., Winston, R., Adeyinka, O., Harkness, A., ... \& Esserman, D. A. (2019). Project ESTEEM protocol: a randomized controlled trial of an LGBTQ-affirmative treatment for young adult sexual minority men's mental and sexual health. BMC Public Health, 19, 1-12. https://doi.org/10.1186/s12889-019-7346-4

Palfai, T. P., Winter, M., Lu, J., Rosenbloom, D., \& Saitz, R. (2014). Personalized feedback as a universal prevention approach for college drinking: A randomized trial of an e-mail linked universal web-based alcohol intervention. The Journal of Primary Prevention, 35, 75-84. https://doi.org/10.1007/s10935-013-0337-9

Park, H., Rodgers, S., McElroy, J. A., \& Everett, K. (2018). Sexual and gender minority’s social media user characteristics: Examining preferred health information. Health Marketing Quarterly, 35, 1-17. https://doi.org/10.1080/07359683.2017.1310553

Parks, C. A. (1999). Lesbian social drinking: The role of alcohol in growing up and living as lesbian. Contemporary Drug Problems, 26, 75-129. https://doi.org/10.1177/009145099902600105

Parks, C. A., \& Heller, N. R. (2013). The influence of early drinking contexts on current drinking among adult lesbian and bisexual women. Journal of the American Psychiatric Nurses Association, 19(5), 241-254. https://doi.org/10.1177/1078390313500145 
Parks, C. A., Hughes, T. L., \& Kinnison, K. E. (2007). The relationship between early drinking contexts of women "coming out" as lesbian and current alcohol use. Journal of LGBT Health Research, 3(3), 73-90. https://doi.org/10.1080/15574090802095823

Parsons, J. T., Rendina, H. J., Moody, R. L., Gurung, S., Starks, T. J., \& Pachankis, J. E. (2017). Feasibility of an emotion regulation intervention to improve mental health and reduce HIV transmission risk behaviors for HIV-positive gay and bisexual men with sexual compulsivity. AIDS and Behavior, 21, 1540-1549. https://doi.org/10.1007/s10461-0161533-4

Pedersen E. R., Helmuth E. D., Marshall G. N., Schell T. L., PunKay M., Kurz J. (2015). Using Facebook to recruit young adult veterans: Online mental health research. JMIR Research Protocols, 4, e63, https://doi.org/10.2196/resprot.3996

Pedersen, E. R., Marshall, G. N., Schell, T. L., \& Neighbors, C. (2016). Young adult veteran perceptions of peers' drinking behavior and attitudes. Psychology of Addictive Behaviors, 30, 39-51. https://doi.org/10.1037/adb0000120

Pedersen, E. R., Parast, L., Marshall, G. N., Schell, T. L., \& Neighbors, C. (2017). A randomized controlled trial of a web-based, personalized normative feedback alcohol intervention for young-adult veterans. Journal of Consulting and Clinical Psychology, 85, 459-470. https://doi.org/10.1037/ccp0000187

Pemberton, M. R., Williams, J., Herman-Stahl, M., Calvin, S. L., Bradshaw, M. R., Bray, R. M., ... Mitchell, G. M. (2011). Evaluation of two web-based alcohol interventions in the U.S. military. Journal of Studies on Alcohol and Drugs, 72, 480-489. https://doi.org/10.15288/jsad.2011.72.480 
Perkins, H. W. (2003). The social norms approach to preventing school and college age substance abuse: A handbook for educators, counselors, and clinicians. San Francisco, CA, US: Jossey-Bass.

Perkins, H. W., \& Berkowitz, A. D. (1986). Perceiving the community norms of alcohol use among students: Some research implications for campus alcohol education programming. International Journal of the Addictions, 21, 961-976. https://doi.org/10.3109/10826088609077249

Phillips, A. S., \& Guarnaccia, C. A. (2020). Self-determination theory and motivational interviewing interventions for type 2 diabetes prevention and treatment: a systematic review. Journal of Health Psychology, 25, 44-66. https://doi.org/10.1177\%2F1359105317737606

Prentice, D. A., \& Miller, D. T. (1993). Pluralistic ignorance and alcohol use on campus: Some consequences of misperceiving the social norm. Journal of Personality and Social Psychology, 64, 243-256. https://doi.org/10.1037/0022-3514.64.2.243

Przybylski, A. K., Rigby, C. S., \& Ryan, R. M. (2010). A motivational model of video game engagement. Review of General Psychology, 14, 154-166. https://doi.org/10.1037/a0019440

Radtke, T., Ostergaard, M., Cooke, R., \& Scholz, U. (2017). Web-based alcohol intervention: Study of systematic attrition of heavy drinkers. Journal of Medical Internet Research, 19, e217. https://doi.org/10.2196/jmir.6780

Raley, A. B., \& Lucas, J. L. (2006). Stereotype or success? Prime-time television's portrayals of gay male, lesbian, and bisexual characters. Journal of Homosexuality, 51, 19-38. https://doi.org/10.1300/J082v51n02_02 
Ramirez-Valles J., Kuhns L. M., Manjarrez, D. (2014). Tal Como Somos/Just As We Are: An educational film to reduce stigma toward gay and bisexual men, transgender individuals, and persons living with HIV/ AIDS. Journal of Health Communication. 19, 478-492. https://doi.org/10.1080/10810730.2013.821555

Ratts M. J., Kaloper M., McReady C., Tighe, L., Butler, S.K., Dempsey, K., McCullough, J. (2013). Safe Space programs in K-12 schools: Creating a visible presence of LGBTQ allies. Journal of LGBT Issues in Counseling, 387-404. https://doi.org10.1080/15538605.2013.839344

Reid, A. E., Carey, K. B., Merrill, J. E., \& Carey, M. P. (2015). Social network influences on initiation and maintenance of reduced drinking among college students. Journal of Consulting and Clinical Psychology, 83, 36-44. https://doi.org/10.1037/a0037634

Reid A. E., Dovidio J. F., Ballester E., Johnson B. T. (2014). HIV prevention interventions to reduce sexual risk for African Americans: The influence of community-level stigma and psychological processes. Social Science \& Medicine, 103,118-125. https://doi.org/10.1016/j.socscimed.2013.06.028.

Resnicow, K., Baranowski, T., Ahluwalia, J. S., \& Braithwaite, R. L. (1999). Cultural sensitivity in public health: defined and demystified. Ethnicity \& Disease, 9, 10-21

Resnicow, K., Soler, R., Braithwaite, R. L., Ahluwalia, J. S., \& Butler, J. (2000). Cultural sensitivity in substance use prevention. Journal of Community Psychology, 28, 271-290. https://doi.org/10.1002/1520-662928

Rigby, S., \& Ryan, R. (2007). The Player Experience of Need Satisfaction (PENS): An applied model and methodology for understanding key components of the player experience. Available at https://natronbaxter.com/wp-content/uploads/2010/05/PENS_Sept07.pdf 
Riggle, E. D., Gonzalez, K. A., Rostosky, S. S., Black, W. W. (2014). Cultivating positive LGBTQA identities: An intervention study with college students. Journal of LGBT Issues in Counseling, 8, 264-281. https://doi.org/10.1080/15538605.2014.933468

Riper, H., van Straten, A., Keuken, M., Smit, F., Schippers, G., \& Cuijpers, P. (2009). Curbing problem drinking with personalized-feedback interventions: a meta-analysis. American Journal of Preventive Medicine, 36, 247-255. https://doi.org/10.1016/j.amepre.2008.10.016.

Rodriguez, L. M., Neighbors, C., Rinker, D. V., Lewis, M. A., Lazorwitz, B., Gonzales, R. G., \& Larimer, M. E. (2015). Remote versus in-lab computer-delivered personalized normative feedback interventions for college student drinking. Journal of Consulting and Clinical Psychology, 83, 455-463. https://doi.org/10.1037/a0039030

Rogers, A. H., Seager, I., Haines, N., Hahn, H., Aldao, A., \& Ahn, W. Y. (2017). The indirect effect of emotion regulation on minority stress and problematic substance use in lesbian, gay, and bisexual individuals. Frontiers in Psychology, 8, e1881. https://doi.org/10.3389/fpsyg.2017.01881

Romero-Canyas, R., Downey, G., Reddy, K. S., Rodriguez, S., Cavanaugh, T. J., \& Pelayo, R. (2010). Paying to belong: When does rejection trigger ingratiation? Journal of Personality and Social Psychology, 99(5), 802-823. https://doi.org/10.1037/a0020013

Rosario, M., Corliss, H. L., Everett, B. G., Reisner, S. L., Austin, S. B., Buchting, F. O., \& Birkett, M. (2014). Sexual orientation disparities in cancer-related risk behaviors of tobacco, alcohol, sexual behaviors, and diet and physical activity: Pooled youth risk behavior surveys. American Journal of Public Health, 104, 245-254.

https://doi.org/10.2105/AJPH.2013.301506 
Ryan, R. M., \& Deci, E. L. (2000). Self-determination theory and the facilitation of intrinsic motivation, social development, and well-being. American Psychologist, 55, 68-78. https://doi.org/10.1037/0003-066X.55.1.68

Ryan, R. M., Rigby, C. S., \& Przybylski, A. (2006). The motivational pull of video games: A self-determination theory approach. Motivation and Emotion, 30, 344-360. https://doi.org/10.1007/s11031-006-9051-8

Sailer, M., Hense, J. U., Mayr, S. K., \& Mandl, H. (2017). How gamification motivates: An experimental study of the effects of specific game design elements on psychological need satisfaction. Computers in Human Behavior, 69, 371-380. https://doi.org/10.1016/j.chb.2016.12.033

Sardi, L., Idri, A., \& Fernández-Alemán, J. L. (2017). A systematic review of gamification in eHealth. Journal of Biomedical Informatics, 71, 31-48. https://doi.org/10.1016/j.jbi.2017.05.011

Saunders, J. B., Kypri, K., Walters, S. T., Laforge, R. G., \& Larimer, M. E. (2004). Approaches to brief intervention for hazardous drinking in young people. Alcoholism: Clinical and Experimental Research, 28, 322-329. https://doi.org/10.1097/01.ALC.0000113418.12889

Saunders, S. M., Zygowicz, K. M., \& D'Angelo, B. R. (2006). Person-related and treatmentrelated barriers to alcohol treatment. Journal of Substance Abuse Treatment, 30, 261-270. https://doi.org/10.1016/j.jsat.2006.01.003

Schuler, M. S., \& Collins, R. L. (2020). Sexual minority substance use disparities: bisexual women at elevated risk relative to other sexual minority groups. Drug and Alcohol Dependence, 206, 107755. https://doi.org/10.1016/j.drugalcdep.2019.107755 
Schulz, D. N., Candel, M. J., Kremers, S. P., Reinwand, D. A., Jander, A., \& de Vries, H. (2013). Effects of a Web-based tailored intervention to reduce alcohol consumption in adults: randomized controlled trial. Journal of Medical Internet Research, 15(9), e206. https://doi.org/10.2196/jmir.2568.

Seaborn, K., \& Fels, D. I. (2015). Gamification in theory and action: A survey. International Journal of Human-Computer Studies, 74, 14-31. https://doi.org/10.1016/j.ijhcs.2014.09.006

Sell, R. L. (1997). Defining and measuring sexual orientation: A review. Archives of Sexual Behavior, 26(6), 643-658. https://doi.org/10.1023/A:1024528427013

Sexton, P., Flores, D., \& Bauermeister, J. (2018). Young sexual minority women's definition of community: Toward addressing health disparities in the LGBTQQ community. Journal of Community Psychology, 46, 133-145. https://doi.org/10.1002/jcop.21929

Sheldon, K. M., \& Filak, V. (2008). Manipulating autonomy, competence, and relatedness support in a game-learning context: New evidence that all three needs matter. British Journal of Social Psychology, 47, 267-283. https://doi.org/10.1348/014466607X238797

Silva, P. J. (2005). Deflecting reactance: The role of similarity in increasing compliance and reducing resistance. Basic and Applied Social Psychology, 27, 277-284. https://doi.org/10.1207/s15324834basp2703_9

Slater, M. E., Godette, D., Huang, B., Ruan, W. J., \& Kerridge, B. T. (2017). Sexual orientationbased discrimination, excessive alcohol use, and substance use disorders among sexual minority adults. LGBT Health, 4, 337-344. https://doi.org/10.1089/lgbt.2016.0117 
Sparkman, G., \& Walton, G. M. (2017). Dynamic norms promote sustainable behavior, even if it is counternormative. Psycological Science, 28, 1663-1674. https://doi.org/10.1177\%2F0956797617719950

Sparkman, G., \& Walton, G. M. (2019). Witnessing change: Dynamic norms help resolve diverse barriers to personal change. Journal of Experimental Social Psychology, 82, 238252. https://doi.org/10.1016/j.jesp.2019.01.007

Steers, M. N., Coffman, A. D., Wickham, R. E., Bryan, J. L., Caraway, L., \& Neighbors, C. (2016). Evaluation of alcohol-related personalized normative feedback with and without an injunctive message. Journal of Studies on Alcohol and Drugs, 77, 337-342. https://doi.org/10.15288/jsad.2016.77.337

Szinay, D., Jones, A., Chadborn, T., Brown, J., \& Naughton, F. (2020). Influences on the uptake of and engagement with health and well-being smartphone apps: Systematic review. Journal of Medical Internet Research, 22, e17572. https://doi.org/10.2196/17572

Talley, A. E. (2013). Recommendations for improving substance abuse treatment interventions for sexual minority substance abusers. Drug and Alcohol Review, 32, 539-540. https://doi.org/10.1111\%2Fdar.12052

Talley, A. E., Gilbert, P. A., Mitchell, J., Goldbach, J., Marshall, B. D., \& Kaysen, D. (2016). Addressing gaps on risk and resilience factors for alcohol use outcomes in sexual and gender minority populations. Drug and Alcohol Review, 35, 484-493. https://doi.org/10.1111/dar.12387

Talley, A. E., Sher, K. J., Steinley, D., Wood, P. K., \& Littlefield, A. K. (2012). Patterns of alcohol use and consequences among empirically derived sexual minority subgroups. 
Journal of Studies on Alcohol and Drugs, 73, 290-302.

https://doi.org/10.15288/jsad.2012.73.290

Taylor, P. (2013). A survey of LGBT Americans: attitudes, experiences and values in changing times. Pew Research Center. Retrieved from https://www.pewsocialtrends.org/2013/06/13/a-survey-of-lgbt-americans/\#

Thomas, D. (2006). A general inductive approach for analyzing qualitative evaluation data. American Journal of Evaluation, 27, 237-246. http://dx.doi.org/10.1177/1098214005283748

Trocki, K. F., Drabble, L., \& Midanik, L. (2005). Use of heavier drinking contexts among heterosexuals, homosexuals and bisexuals: results from a National Household Probability Survey. Journal of Studies on Alcohol, 66, 105-110.

Turner, J. C., Hogg, M. A., Oakes, P. J., Reicher, S. D., \& Wetherell, M. S. (1987). Rediscovering the social group: A self-categorization theory. Cambridge, MA, US: Basil Blackwell.

Turner, J. C., \& Reynolds, K. J. (2011). Self-categorization theory. In Handbook of Theories in Social Psychology: Vol. 2(1) (pp. 399-417). https://doi.org/10.4135/9781446249222.n46

Uysal, A., Yildirim, I. G., \& Bostan, B. (2016). Self-determination theory in digital games. In B. Boston (Ed.) International Series on Computer Entertainment and Media Psychology Gamer Psychology and Behavior (pp. 123-135). Cham, Switzerland: Springer International Publishing.

Vaeth, P. A., Wang-Schweig, M., \& Caetano, R. (2017). Drinking, alcohol use disorder, and treatment access and utilization among US racial/ethnic groups. Alcoholism: Clinical and Experimental Research, 41, 6-19. https://doi.org/10.1111/acer.13285 
van der Spek, E. D. (2012). Towards designing for competence and engagement in serious games. In M. Ma, M. F. Oliveira, J. B. Hauge, H. Duin, \& K.-D. Thoben (Eds.), Serious Games Development and Applications (pp. 98-109). Springer Berlin Heidelberg.

Veldhuis, C. B., Hughes, T. L., Drabble, L., Wilsnack, S. C., Riggle, E. D., \& Rostosky, S. S. (2019). Relationship status and drinking-related outcomes in a community sample of lesbian and bisexual women. Journal of Social and Personal Relationships, 36, 244-268. https://doi.org/10.1177\%2F0265407517726183

Vinacke, W. E. (1957). Stereotypes as social concepts. The Journal of Social Psychology, 46, 229-243.

Walters, S. T., \& Neighbors, C. (2005). Feedback interventions for college alcohol misuse: What, why and for whom? Addictive Behaviors, 30, 1168-1182. https://doi.org/10.1016/j.addbeh.2004.12.005

Walters, S. T., \& Woodall, W. G. (2003). Mailed feedback reduces consumption among moderate drinkers who are employed. Prevention Science, 4, 287-294. https://doi.org/10.1023/A:1026024400450

Walton, G. M. (2014). The new science of wise psychological interventions. Current Directions in Psychological Science, 23, 73-82. https://doi.org/10.1177\%2F0963721413512856

Walton, G. M., \& Wilson, T. D. (2018). Wise interventions: Psychological remedies for social and personal problems. Psychological Review, 125, 617-655. https://psycnet.apa.org/doi/10.1037/rev0000115

Walton, G. M., \& Yeager, D. S. (2020). Seed and soil: Psychological affordances in contexts help to explain where wise interventions succeed or fail. Current Directions in Psychological Science, 29, 219-226. https://doi.org/10.1177\%2F0963721420904453 
Weber (2008) Using to numb the pain: Substance use and abuse among lesbian, gay and bisexual individuals. Journal of Mental Health Counseling, 30, 31-48.

https://doi.org/10.17744/mehc.30.1.2585916185422570

Weibel, D., Wissmath, B., Habegger, S., Steiner, Y., \& Groner, R. (2008). Playing online games against computer- vs. human-controlled opponents: Effects on presence, flow, and enjoyment. Computers in Human Behavior, 24, 2274-2291.

https://doi.org/10.1016/j.chb.2007.11.002

Weiss, J. T. (2004). GL vs. BT: The archaeology of biphobia and transphobia within the US gay and lesbian community. Journal of Bisexuality, 3, 25-55. https://doi.org/10.1300/J159v03n03_02

Welzer-Lang, D. (2008). Speaking out loud about bisexuality: Biphobia in the gay and lesbian community. Journal of Bisexuality, 8, 81-95. https://doi.org/10.1080/15299710802142259

White, I. R., Horton, N. J., Carpenter, J., \& Pocock, S. J. (2011). Strategy for intention to treat analysis in randomised trials with missing outcome data. British Medical Journal, 342, d40. https://doi.org/ 10.1136/bmj.d40

White, I. R., Carpenter, J., \& Horton, N. J. (2012). Including all individuals is not enough: lessons for intention-to-treat analysis. Clinical Trials, 9, 396-407. https://doi.org/10.1177/1740774512450098

Willson, M., \& Leaver, T. (2017). Social, Casual and Mobile Games: The Changing Gaming Landscape. Camden, UK: Bloomsbury Academic:

Wilsnack, S. C., Hughes, T. L., Johnson, T. P., Bostwick, W. B., Szalacha, L. A., Benson, P.,... \& Kinnison, K. E. (2008). Drinking and drinking-related problems among heterosexual 
and sexual minority women. Journal of Studies on Alcohol and Drugs, 69, 129-139. https://doi.org/10.15288/jsad.2008.69.129

Wilson, S. M., Gilmore, A. K., Rhew, I. C., Hodge, K. A., \& Kaysen, D. L. (2016). Minority stress is longitudinally associated with alcohol-related problems among sexual minority women. Addictive Behaviors, 61, 80-83. https://doi.org/10.1016/j.addbeh.2016.05.017

Witkiewitz, K., Falk, D. E., Kranzler, H. R., Litten, R. Z., Hallgren, K. A., O'Malley, S. S., In conjunction with the Alcohol Clinical Trials Initiative (ACTIVE) Workgroup. (2014). Methods to analyze treatment effects in the presence of missing data for a continuous heavy drinking outcome measure when participants drop out from treatment in alcohol clinical trials. Alcoholism: Clinical and Experimental Research, 38, 2826-2834. https://doi.org/10.1111\%2Facer.12543

World Health Organization (2014). Global status report on alcohol and health; WHO Press: Geneva, Switzerland. https://www.who.int/substance_abuse/publications/alcohol_2014/en/ Young, C. M., Neighbors, C., DiBello, A. M., Sharp, C., Zvolensky, M. J., \& Lewis, M. A. (2016). Coping motives moderate efficacy of personalized normative feedback among heavy drinking US college students. Journal of Studies on Alcohol and Drugs, 77, 495499. https://doi.org/10.15288/jsad.2016.77.495.

Young, C. M., Pedersen, E. R., Pearson, A. D., \& Neighbors, C. (2018). Drinking to cope moderates the efficacy of changing veteran drinking norms as a strategy for reducing drinking and alcohol-related problems among US veterans. Psychology of Addictive Behaviors, 32, 213-223. https://psycnet.apa.org/doi/10.1037/adb0000347 